\title{
Cooperative Coordination and Formation Control for Multi-agent Systems
}

Zhiyong Sun

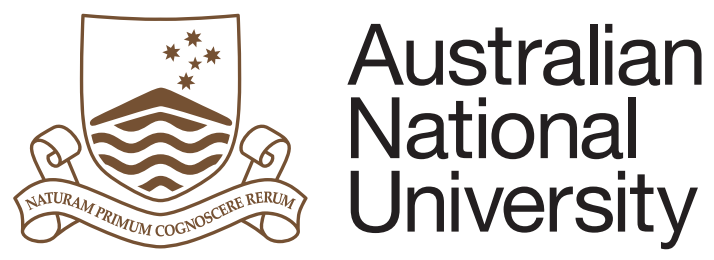

A thesis submitted for the degree of Doctor of Philosophy

The Australian National University

February 2017 
(C) Zhiyong Sun 2017

All Rights Reserved. 
Except where otherwise indicated, this thesis is my own original work. The nature and extent of collaboration have been outlined in this thesis.

zhigony Sun 孙志尊 Zhiyong Sun

31 January 2017 

To my family. 
"From a hundred rabbits you can't make a horse, and a hundred suspicions don't make a proof..."

—Fyodor Dostoyevsky: Crime and Punishment (Chapter II of Part VI).

"To begin well is common;

To end well is rare indeed."

—The Ancient Chinese Classic of Poetry ${ }^{1}$ (Shi-jing, published c. 600 BC).

\footnotetext{
${ }^{1}$ Translated from old Chinese by Arthur Waley.
} 


\section{Declaration}

My doctoral studies have been conducted under the guidance and supervision of Prof. Brian D. O. Anderson, Assoc. Prof. Changbin (Brad) Yu and Dr. Adrian N. Bishop. This thesis contains no material which has been accepted for the award of any other degree or diploma in any university.

Most of the results in this thesis have been published at refereed international journals or international conferences. Some of these results have been achieved in collaboration with other researchers, or carried out in other institutions. I have included as part of the dissertation a statement clearly outlining the extent of collaboration, with whom and under what auspices. Coauthored publications as a result of parts of my thesis research are listed as below: (Note: (i) Submitted papers that are under review are not listed here; (ii) * indicates that the thesis incorporates material of this paper. )

\section{Refereed Journal Papers}

1. * Z. Sun, S. Mou, B. D. O. Anderson, and A. S. Morse. Rigid motions of 3-D undirected formations with distance mismatch. IEEE Transactions on Automatic Control, conditionally accepted, 2016.

2. * Z. Sun, M.-C. Park, B. D. O. Anderson, and H.-S. Ahn. Distributed stabilization control of rigid formations with prescribed orientation. Automatica, vol. 78, No. 4, pp.250-257, 2017. Full version available at arXiv http: //arxiv.org/abs/1606.03199.

3. M. H. Trinh, V. H. Pham, M.-C. Park, Z. Sun, B. D. O. Anderson, and H.S. Ahn. Comments on 'Global stabilization of rigid formations in the plane'. Automatica, in press, 2016. DOI: 10.1016/j.automatica.2016.11.006

4. * Z. Sun, B. D. O. Anderson, M. Deghat, and H.-S. Ahn. Rigid formation control of double-integrator systems. International Journal of Control. In press. DOI: 10.1080/00207179.2016.1207100, 2016

5. * Z. Sun, S. Mou, B. D. O. Anderson, and M. Cao. Exponential stability for formation control systems with generalized controllers: a unified approach. Systems and Control Letters, vol. 93, pp.50-57, 2016.

6. Z. Sun, S. Mou, M. Deghat, and B. D. O. Anderson. Finite time distributed distance-constrained shape stabilization and flocking control for d-dimensional undirected rigid formations. International Journal of Robust and Nonlinear Control, vol. 26, No. 13, pp.2824-2844, 2016. 
7. S. Mou, M. A. Belabbas, A. S. Morse, Z. Sun, B. D. O. Anderson. Undirected Rigid Formations are Problematic. IEEE Transactions on Automatic Control, vol. 61, No. 10, pp.2821-2836, 2016.

\section{Refereed Conference Papers}

8. * Z. Sun, H. Garcia de Marina, B. D. O. Anderson, and M. Cao. Quantization effects in rigid formation control. Proc. of the 6th Australian Control Conference (AUCC'16), pp. 168-173, Newcastle, Australia, 2016.

9. X. Zhang, Z. Sun, and C. Yu. Finite-time synchronization of networked Kuramoto oscillators. Proc. of the 6th Australian Control Conference (AUCC'16), pp. 81-86, Newcastle, Australia, 2016.

10. ${ }^{*}$ Z. Sun and B. D. O. Anderson. Formation feasibility on coordination control of networked heterogeneous systems with drift terms. Proc. of the 55th IEEE Conference on Decision and Control (CDC'16), pp. 3462-3467, Las Vegas, USA, 2016.

11. C. Lageman and Z. Sun. Consensus on spheres: convergence analysis and perturbation theory. Proc. of the 55th IEEE Conference on Decision and Control (CDC'16), pp. 19-24, Las Vegas, USA, 2016.

12. Z. Sun, N. Huang, B. D. O. Anderson and Z. Duan. A new distributed Zenofree event-triggered algorithm for multi-agent consensus. Proc. of the 55th IEEE Conference on Decision and Control (CDC'16), pp. 3444-3449, Las Vegas, USA, 2016.

13. M.-C. Park, Z. Sun, B. D. O. Anderson, and H.-S. Ahn. Distance-based control of K4 formation with almost global convergence. Proc. of the 55th IEEE Conference on Decision and Control (CDC'16), pp. 904-909, Las Vegas, USA, 2016. Also available at arXiv: http://arxiv.org/abs/1606.01638.

14. M. H. Trinh, M.-C. Park, Z. Sun, B. D. O. Anderson, V. H. Pham, and H.-S. Ahn. Further Results in Rigidity Index. Proc. of the 55th IEEE Conference on Decision and Control (CDC'16), pp. 922-927, Las Vegas, USA, 2016.

15. * Z. Sun, U. Helmke and B. D. O. Anderson. Rigid formation shape control in general dimensions: an invariance principle and open problems. Proc. of the 54th IEEE Conference on Decision and Control (CDC'15), pp. 6095- 6100, Osaka, Japan, 2015 (Best Student Paper Award Finalist)

16. ${ }^{*}$ Z. Sun and B. D. O. Anderson. Rigid formation control systems modelled by double integrators: system dynamics and convergence analysis. Proc. of the 2015 Australian Control Conference (AUCC'15), pp. 241-246, Gold Coast, Australia, 2015 (Best Student Paper Award Winner) 
17. Z. Sun, G. S. Seyboth and B. D. O. Anderson. Collective control of multiple unicycle agents with non-identical constant speeds: Tracking control and performance limitation. Proc. of the 2015 IEEE Multi-Conference on Systems and Control (MSC'15), pp. 1361-1366, Sydney, Australia, 2015

18. * Z. Sun and B. D. O. Anderson. Rigid formation control with prescribed orientation. Proc. of the 2015 IEEE Multi-Conference on Systems and Control (MSC'15), pp. 639-645, Sydney, Australia, 2015

19. Z. Sun, C. Yu and B. D. O. Anderson. Distributed optimization on proximity network rigidity via robotic movements. Proc. of the 34th Chinese Control Conference (CCC'15), pp. 6954-6960, Hangzhou, China, 2015. Also available at arXiv: http://arxiv.org/abs/1309.4850.

20. Q. Liu, Z. Sun, J. Qin and C. Yu. Distance-based formation shape stabilisation via event-triggered control. Proc. of the 34th Chinese Control Conference (CCC'15), pp. 6948-6953, Hangzhou, China, 2015

21. Z. Sun, Q. Liu, C. Yu and B. D. O. Anderson. Generalized controllers for rigid formation stabilization with application to event-based controller design. Proc. of the European Control Conference (ECC'15), pp. 217-222, Linz, Austria, 2015

22. B. Jiang, Z. Sun and B. D. O. Anderson. Higher order Voronoi based mobile coverage control. Proc. of the American Control Conference (ACC'15), pp. 1457-1462, Chicago, USA. Also available at arXiv preprint http://arxiv.org/abs/ 1410.1941 .

23. Z. Sun, S. Mou, U. Helmke, and B. D. O. Anderson. Convergence Analysis for Rigid Formation Control with Unrealizable Shapes: The 3 Agent Case. Proc. of the 4th Australian Control Conference(AUCC'14), Canberra, Australia, pp. 1-6, 2014 (Best Student Paper Award Finalist)

24. M.-C. Park, Z. Sun, K.-K. Oh, B. D. O. Anderson, and H.-S. Ahn. Finite-Time Convergence Control for Acyclic Persistent Formations. Proc. of the 2014 IEEE Multi-conference on Systems and Control (MSC'14), Nice/Antibes, France, pp. 1608-1613, 2014

25. M.-C. Park, Z. Sun, B. D. O. Anderson, and H.-S. Ahn. Stability Analysis on Four Agent Tetrahedral Formations. Proc. of the 53rd IEEE Conference on Decision and Control(CDC'14), Los Angeles, USA, pp. 631-636, 2014

26. U. Helmke, S. Mou, Z. Sun, and B. D. O. Anderson. Geometrical Methods for Mismatched Formation Control. Proc. of the 53rd IEEE Conference on Decision and Control(CDC'14), Los Angeles, USA, pp. 1341-1346, 2014

27. * Z. Sun, S. Mou, B. D. O. Anderson, and A. S. Morse. Formation movements in minimally rigid formation control with mismatched mutual distances. Proc. of the 53rd IEEE Conference on Decision and Control(CDC'14), Los Angeles, USA, pp.6161-6166, 2014 
28. Z. Sun, S. Mou, M. Deghat, B. D. O. Anderson, and A. S. Morse. Finite Time Distance-based Rigid Formation Stabilization and Flocking. Proc. of the 19th IFAC World Congress (IFAC WC'14), Cape Town, South Africa, pp. 9183-9189, 2014.

29. * Z. Sun, S. Mou, B. D. O. Anderson, and A. S. Morse. Non-Robustness of Gradient Control of 3-D Formations with Distance Mismatch. Proc. of the 3rd Australian Control Conference (AUCC 2013), Perth, Australia, pp.369-374, 2013. 


\section{Acknowledgments}

I have so many people to thank for their great help during my PhD journey! My sincerest gratitude first goes to my principal supervisor, Prof. Brian D. O. Anderson, for your caring supervision, patient guidance, and countless discussions for my whole $\mathrm{PhD}$ research and study. Brian has been a role model for me to follow, not only in research but also in life and many other ways. His encouragement and extraordinarily conscientious mentoring (which enables me to deeply appreciate the meaning of the word Doktorvater), as well as his warm and good humoured personality have made my PhD journey a really enjoyable one under his wing. I have always been feeling extremely lucky to be one of your students, and been greatly honoured to be the last $\mathrm{PhD}$ student receiving your primary supervision. I also want to thank Brian's wife, Mrs. Dianne Anderson, for the great hospitality and the very nice Australian-style dishes that I have enjoyed very much at Brian's home in Canberra and at Brian's beach house in Malua Bay.

I wish to express my heartfelt thanks to my co-advisors, Assoc. Prof. Changbin (Brad) Yu and Dr. Adrian N. Bishop, for your great help and support along my research journey. My way of becoming a well-trained researcher is due to your careful guidance as well. I shall in particular thank Brad for lots of help during my pre-PhD study. I was "recruited" by Brad in 2012, after I obtained a Master Degree in China and before I decided to come to Australia to pursue a PhD degree. Brad's support and advice on my application to the ANU PhD program and in particular to the Endeavour Award have contributed a lot to the success, which then paved a smooth way for me to start a $\mathrm{PhD}$ adventure at ANU.

In my PhD journey, I have been fortunate to have the privilege of receiving guidance and supervision of world-class researchers from other institutes. Prof. A. Stephen Morse from Yale University deserves my truest admiration. His critical thinking, the rigour in mathematical proofs, and the very keen sense in research problems have influenced me a lot, and will be a life-long treasure for my future career. I would also pay my deep respect to the late Prof. Uwe Helmke from University of Würzburg. I have enjoyed a lot from discussions and collaborations with him. His enthusiasm in research, always surprising insights in tackling a research problem and his vast knowledge in mathematics have benefited me a lot and also enabled me to further appreciate the true beauty of math.

I will also take this opportunity to thank all my collaborators. Prof. Hyo-Sung Ahn from Gwangju Institute of Science and Technology (GIST), South Korea, and Prof. Ming Cao from University of Groningen, The Netherlands, thank you for all your patient guidance and discussions. My other collaborators have also greatly helped me go though my PhD time, and have shaped me from a fresh PhD student 
to a PhD graduate who now has something to report in this thesis. Without their help and collaborations I surely cannot achieve what now I have. They are: Dr. Shaoshuai Mou (Purdue University, USA), Dr. Christian Lageman (University of Würzburg, Germany), Dr. Héctor Garcia de Marina (École nationale de l'aviation civile, France), Mr. Myoung-Chul Park (GIST, South Korea), Dr. Mohammad Deghat (University of New South Wales, now at Data61 of CSIRO), Mr. Minh Hoang Trinh (GIST, South Korea), Dr. Shiyu Zhao (University of Sheffield, UK), Dr. Georg S. Seyboth (University of Stuttgart, Germany), Prof. Jiahu Qin (University of Science and Technology of China), Dr. Na Huang (Peking University, now at Hangzhou Dianzi University, China), and Mr. Bomin Jiang (MIT). The discussions with you on different research topics are always very stimulating and have been a constant source of inspirations. Furthermore, in many of our group meetings I have the privilege to discuss technical issues and research problems directly related to applications with DSTG (Defence Science and Technology Group) researchers. Their insightful feedbacks and comments are also greatly appreciated.

In various occasions including research meetings and conference attendances, I have the chance to meet other leading researchers in the field. Dr. Guodong Shi, Prof. Zhiyun Lin, Prof. Wei Ren, Prof. Wei Xing Zheng, Prof. Daniel Zelazo, Dr. Xudong Chen, Dr. Junfeng Wu, Dr. Ji Liu, Dr. Iman Shames and many others, thanks for being an example of a leading academic for me!

One of the most important parts in my PhD life should be the DICE group at the Research School of Engineering at ANU. I am grateful that I have been accompanied by so many nice and brilliant members and visitors in our group: Junming, Qingchen, Xiaolei(Eric), Yun(Gavin), Mengbin(Ben), YonHon, Zhixun, Xianwei, and many others. Without you, my PhD life at Canberra would surely be of much less fun! Interactions with academics and students from other research groups at the same RSISE Building (now Brian Anderson Building), including Dr. Hongdong Li, Dr. Jochen Trumpf, Dr. Nan Yang, Dr. Yuchao Dai, Mr. Geoffrey Stacey et al., are always very enjoyable and inspiring! In addition, the excellent administration support from the department administrators at CECS also deserves my special thanks.

I consider myself a fortunate $\mathrm{PhD}$ student to obtain certain opportunities of visiting some leading research groups in the world in the past three years. For each visit I received lots of help from the fellow students and group members at the host group and I appreciate a lot for their support. They are: Lili, Fengjiao (Zoe), Daniel from Yale University; Sung-Mo, Myoung-Chul and other DCASL lab mates from GIST; Qingkai, Yuzhen, Xiaodong, Jie and other members from ENTEG group at the University of Groningen; Michael and other members from Prof. Uwe Helmke's group at University of Würzburg; and those fellow students from Prof. Toshiharu Sugie's group at University of Kyoto. Research visits to these institutes not only have enhanced my research productivity, but also have consisted of some very beautiful travel memory to me.

In the past three plus years I have been living at No. 8 of Bagot St., and have enjoyed a lot, among other things, the nice environment and interesting chatting with my landlady and housemates. Thank you, Pamela and Rémi! You mean so 
much to my life at Canberra.

My life at Canberra has also been enriched by becoming a member of Woden Daybreak Rotary Club. This is a truly family-like club, and I consider joining the club as one of the best decisions I have ever made after I moved to Canberra. Thank you, Rosemary, Patty, Robyn $(\times 2)$, Rob, Greg, Mark, Darren, John, Gavin, Lyn, Barry, Karen, Alison, Graham, Sue and many other rotarians! The community service and various voluntary activities have been an indispensable part of my Canberra life (apart from my ANU PhD life), which have also provided me numerous unique opportunities to enjoy the Canberra local culture!

My old friends in China should also have a place in my acknowledgement. Haixiang, Shanshan, Peizhi, Shouzhi and so many old friends in Nanjing, Xi'an, Shanghai and Jinan have been an important part for my memory of my life in China. Special thanks to Xinrui for your understanding and being a company along the way.

My PhD research has been supported by a special scholarship which has a long name: The Prime Minister's Australia Asia Incoming Endeavour Postgraduate Award from Australian Government. The financial support from this scholarship is greatly appreciated. The Endeavour supporting team (Scope Global) has helped to address various issues relating to my settling down at Canberra, international travels and my overall overseas life. In particular, I am thankful to my case manager, Ms. Beth Mutton (Scope Global), for her constant support and professional service in the past three years.

Lastly but also most importantly, I thank my family for always standing with me for so many years. My parents, my younger brother Zhigang and younger sister Yanting, and all my cousins in the large family, have always been the most important part in my life. This thesis is dedicated to my family. 



\section{Abstract}

The primary aim of this thesis is to study cooperative coordination control and formation control for multi-agent systems, with a focus on distributed stabilization control of rigid formation shapes. We consider several problems in the field, ranging from the equilibrium and stability of formation control systems, some practical considerations in formation control, and cooperative coordination control when agents have general dynamical models.

In the first part of the thesis, we study in detail the equilibrium property of rigid formation control systems. A rank-preserving property is established for this coordination control system, and with this property we further prove the instability of a special equilibrium set (termed degenerate equilibria) at which agents' positions only span an affine space with dimension less than that of the full space. The exponential stability of rigid formation control systems for a large family of formation controllers is also proved, with the property applying for both minimally rigid formations and non-minimally rigid formations. This approach provides a general and unified way for stability analysis of formation control systems.

In the second part, we investigate several practical issues on formation control, including robustness issues, rigid shape stabilization with a prescribed orientation, and formation control with quantized measurements. From the exponential stability proved in the first part, we discuss the convergence and robustness property for 3-D rigid formation control systems with distance mismatches, and identify a helical rigid motion induced by mismatched distances. In addition, we propose a feasible formation controller to achieve a desired rigid shape and a prescribed formation orientation in ambient 2-D and 3-D spaces, with minimal knowledge of the global coordinate frame orientation. Furthermore, quantization effects on rigid formation shape stabilization are discussed in detail in the case that the cooperative formation control only uses quantized distance measurements.

In the third part, we extend some main results considered in previous chapters on formation control systems modelled by single integrators to systems modelled by more general dynamics, including double integrator models and nonlinear control systems. First, two types of double-integrator cooperative control systems (i.e. formation stabilization systems and flocking control systems with a target rigid shape) are thoroughly investigated. By using a family of parameterized Hamiltonian-like systems, we further establish certain invariance principles concerning the equilibrium set and local stability, which build the link between the stability analysis for formation systems modelled by single integrators and those modelled by double integrators. In addition, we consider a fundamental problem termed formation feasibility in multiagent cooperative control. The problem concerns general forms of both formation constraints and individual agent's kinematics constraints. In this cooperative control 
framework, we assume each agent is modelled by an affine system with possible drift terms, and the network consists of multiple heterogeneous agents which could have totally different dynamics. Via tools from nonlinear control and differential geometry, an algebraic condition is provided to determine the existence of feasible formations for such heterogeneous networked systems, and a systematic procedure is proposed to generate feasible formations if they exist. 


\section{Contents}

Declaration vii

Acknowledgments $\quad$ xi

$\begin{array}{ll}\text { Abstract } & \text { xv }\end{array}$

1 Introduction 1

1.1 Research background: cooperative control and networked systems . . . 1

1.2 Introduction and literature review . . . . . . . . . . . . . . . 2

1.2.1 Different approaches on formation shape control . . . . . . . 2

1.2.2 Coordinate frame requirement in formation control . . . . . . . 3

1.2.3 Equilibrium and stability analysis on rigid formation control systems .................... 4

1.2.4 Some practical issues in rigid formation control . . . . . . . . . 4

1.2.5 Agent dynamical model: beyond single-integrator models . . . . 5

1.3 Thesis outline and statements of collaborations . . . . . . . . . . . 6

2 Theoretical preliminaries $\quad 9$

2.1 Notations . . . . . . . . . . . . . . . . . . . . . 9

2.2 Preliminary on graph theory . . . . . . . . . . . . . 10

2.3 Preliminary on rigidity theory $\ldots \ldots \ldots \ldots$

2.3.1 Definitions and properties . . . . . . . . . . . . . 10

2.3.2 Rigidity matrix and related results . . . . . . . . . . . . . . 11

2.4 Gradient systems . . . . . . . . . . . . . . . . . . . . . . . . . 14

2.4.1 Definitions and properties . . . . . . . . . . . . . . . . 14

2.4.2 Real analyticity and local minimum . . . . . . . . . . . 15

2.5 Gradient descent control law for rigid formation stabilization . . . . . . 15

I Formation Control Systems: Equilibrium and Stability Analysis $\quad 17$

3 Invariance principles and equilibrium analysis for formation shape control $\begin{array}{ll}\text { systems } & 19\end{array}$

3.1 Introduction . . . . . . . . . . . . . . . . . . . . . . 19

3.1.1 Background and related work . . . . . . . . . . . . . . . 19

3.1 .2 Chapter organization . . . . . . . . . . . . . . . . 20

3.2 Problem setup and motion equations $\ldots \ldots \ldots 21$ 
3.3 Rank-preserving property for formation systems . . . . . . . . . . . . . . 21

3.3.1 Proof of the rank-preserving property . . . . . . . . . . . 21

3.3.2 Consequences of the rank-preserving property $\ldots \ldots \ldots 23$

3.4 Stability analysis for degenerate equilibria . . . . . . . . . . . 24

3.4 .1 The equilibrium set . . . . . . . . . . . . . 26

3.4.2 Eigenvalue property of $E$ and the Hessian $\ldots \ldots \ldots 27$

3.4 .3 Degenerate equilibria are unstable . . . . . . . . . . . 28

3.5 Concluding remarks . . . . . . . . . . . . . . . . . 32

3.6 Appendix: background on rank-preserving matrix flow $\ldots \ldots 32$

4 Exponential stability for formation control systems with generalized controllers 35

4.1 Introduction . . . . . . . . . . . . . . . . . . . 35

4.1.1 Background and related work . . . . . . . . . . . 35

4.1.2 Chapter organization . . . . . . . . . . . . . 37

4.2 Exponential stability for minimally rigid formations . . . . . . . . . . 37

4.2.1 Review of special controllers in the literature . . . . . . . . . . 38

4.2 .2 Convergence analysis . . . . . . . . . . . . . . . 39

4.2.3 Properties of the formation control system with generalized

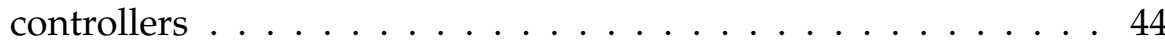

4.3 Exponential stability for non-minimally rigid formations . . . . . . . . 46

4.4 Simulation examples . . . . . . . . . . . . . . . . . . . 49

4.5 Concluding remarks $\ldots \ldots \ldots \ldots$. . . . . . . . . . . . . 52

II Formation Control Systems: Some Practical Considerations 53

5 Robustness issues for 3-D undirected formations with distance mismatches 55

5.1 Introduction . . . . . . . . . . . . . . . 55

5.1 .1 Background and related work . . . . . . . . . . 55

5.1 .2 Chapter organization . . . . . . . . . . . . . . 57

5.2 Motion equations with distance mismatches . . . . . . . . . . . . 57

5.3 Analysis of convergence and formation movements . . . . . . . . . . . 61

5.3.1 Self-contained distance error equation and exponential convergence . . . . . . . . . . . . . . . . 61

5.3 .2 Convergence to a rigid formation . . . . . . . . . . . 62

5.3.3 Rigid motions induced by distance mismatches . . . . . . . . . 62

5.3.4 Convergence of the equilibrium motion . . . . . . . . . . 65

5.4 Further analysis of the rigid helical motions . . . . . . . . . 68

5.4.1 Determining helical motion parameters . . . . . . . . . . 68

5.4 .2 Steering formations by manipulating mismatches . . . . . . . 70

5.5 Concluding remarks . . . . . . . . . . . . . . . 71 
6 Distributed stabilization control of rigid formations with prescribed orien$\begin{array}{ll}\text { tations } & 73\end{array}$

6.1 Introduction . . . . . . . . . . . . . . . 73

6.1 .1 Background and related work . . . . . . . . . 73

6.1 .2 Chapter organization . . . . . . . . . . . . . . 75

6.2 Preliminaries and problem setup . . . . . . . . . . . 75

6.2.1 Rigidity matrix and its null space . . . . . . . . . . 75

6.2.2 Gradient-based formation controller and problem formulation . 76

6.3 Main result . . . . . . . . . . . . . . . . . . . 76

6.3.1 Target formation and control framework . . . . . . . . 76

6.3.2 Controller design . . . . . . . . . . . . . . . . . 79

6.3.3 Properties of the formation control system . . . . . . . . . . 80

6.3 .4 Convergence analysis . . . . . . . . . . . . . 80

6.4 Illustrative examples . . . . . . . . . . . . . . . . . . . 85

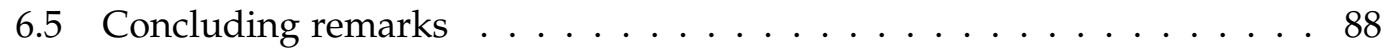

6.6 Appendix: proofs of several lemmas . . . . . . . . . . . . . 88

7 Quantization effects in rigid formation control 91

7.1 Introduction . . . . . . . . . . . . . . . . . . 91

7.1.1 Background, motivation and related work . . . . . . . . 91

7.1 .2 Chapter organization . . . . . . . . . . . . . 92

7.2 Preliminaries . . . . . . . . . . . . . . . . . . . 993

7.2.1 Additional notations in this chapter . . . . . . . . . . . . . 93

7.2.2 Quantizer functions . . . . . . . . . . . . . . . 93

7.2.2.1 Definition of the quantizers . . . . . . . . . . . 93

7.2.2.2 Properties of the quantizers . . . . . . . . . . 94

7.2 .3 Nonsmooth analysis . . . . . . . . . . . . . . . . . . . . . 95

7.3 Formation control with quantized measurements . . . . . . . . . . . 95

7.3.1 Quantized formation controllers . . . . . . . . . . . . 95

7.3.2 Properties of quantized formation control systems . . . . . . 97

7.3.3 Convergence analysis . . . . . . . . . . . . . . . . 99

7.4 A special quantizer: formation control with binary distance information 101

7.4.1 Rigid formation control with coarse measurements . . . . . . . . 101

7.4 .2 Convergence analysis . . . . . . . . . . . . . . . . 102

7.5 Asymmetric uniform quantizer . . . . . . . . . . . . . . . . . . . 104

7.5.1 Motivating example: two-agent formation case . . . . . . . . 104

7.5.2 General formation case . . . . . . . . . . . . . . . . 105

7.6 Illustrative examples and simulations . . . . . . . . . . . . . 105

7.7 Concluding remarks . . . . . . . . . . . . . . . . . . 106

7.8 Appendix: background and useful tools on nonsmooth analysis . . . . . 108 
$\begin{array}{lll}8 & \text { Rigid formation control of double-integrator systems } & 113\end{array}$

8.1 Introduction . . . . . . . . . . . . . . . . . . . . . 113

8.1.1 Background and related work . . . . . . . . . . . . 113

8.1.2 Chapter organization . . . . . . . . . . . . . . . . . . . . 115

8.2 Motion equations . . . . . . . . . . . . . . . . . . . 115

8.2.1 Introduction of double-integrator formation control systems . . 115

8.2.2 Independence of global coordinate frame . . . . . . . . . . . . 117

8.3 System dynamics . . . . . . . . . . . . . . . . . . . . 118

8.3.1 Equilibrium sets for single- and double-integrator formation systems . . . . . . . . . . . . . . . . 118

8.3.2 General results on convergence analysis . . . . . . . . . . . . 121

8.3 .3 Jacobian matrix analysis . . . . . . . . . . . . . . . . . 122

8.4 Perspectives from a parameterized double-integrator formation system 124

8.4 .1 Invariance principles . . . . . . . . . . . . . . . . . 125

8.4.2 Relating double-integrator formation systems to single-integrator formation systems . . . . . . . . . . . . . . 126

8.4.3 Exponential convergence of double-integrator formation systems 130

8.5 Extensions to double-integrator flocking systems . . . . . . . . . . . 131

8.6 Illustrative examples on convergence . . . . . . . . . . . . . . . 132

8.7 Concluding remarks . . . . . . . . . . . . . . . . . . 134

9 Formation feasibility and motion generation of networked heterogeneous $\begin{array}{ll}\text { systems } & 137\end{array}$

9.1 Introduction . . . . . . . . . . . . . . . . . . . 137

9.1.1 Background, motivation and related work . . . . . . . . . . 137

9.1.2 Chapter organization . . . . . . . . . . . . . . . . . . . 139

9.2 Preliminaries, problem formulation and motivating examples . . . . . 139

9.2.1 Preliminaries on differential geometry and system equation . . . 139

9.2 .2 Motivating examples . . . . . . . . . . . . . . . . . . . . . . . . 140

9.2.3 Problem formulation: formation feasibility with kinematics and formation constraints . . . . . . . . . . . . . . . 141

9.3 Conditions for feasible motion . . . . . . . . . . . . . . . . . . . 142

9.3.1 Constraints on inter-agent formations . . . . . . . . . . . . 142

9.3.2 Constraints on individual agents' kinematics . . . . . . . . . . . 142

9.3.3 Main results on formation feasibility . . . . . . . . . . . . 143

9.4 Application to coordination control of multiple constant-speed agents . 144

9.4.1 Affine distribution and codistribution generated by constantspeed dynamics . . . . . . . . . . . . . . . . . . . 144

9.4.2 Example: rigid formation maintenance and coordination by constant-speed agents . . . . . . . . . . . . . . 145

9.5 Motion generation and coordination control with heterogeneous agents 147

9.5.1 Generalization and motion derivation . . . . . . . . . . . . . . . 147

9.5.2 Illustrative example: coordination control with a group of heterogeneous agents . . . . . . . . . . . . 147 
9.6 Concluding remarks . . . . . . . . . . . . . . . . . . . . 151

$\begin{array}{ll}10 \text { Conclusions and future work } & 153\end{array}$

10.1 Thesis summary and contributions . . . . . . . . . . . . . 153

10.1.1 Formation control systems: equilibrium and stability analysis . . 153

10.1.2 Formation control systems: some practical considerations . . . . 154

10.1.3 Distributed coordination control: general system models . . . . . 154

10.2 Future work . . . . . . . . . . . . . . . . . . . . . 155

10.2.1 Equilibrium analysis . . . . . . . . . . . . . . . 155

10.2.2 Robustness issues . . . . . . . . . . . . . . . . . . . 156

10.2.3 Formation feasibility and motion generation . . . . . . . . . . 157

10.2.4 Longer-term research problems: coordination control in non-

linear spaces . . . . . . . . . . . . . . . . . . 157 


\section{List of Figures}

1.1 An overview of relations between the main chapters. . . . . . . . . . 8

2.1 Examples of rigid and non-rigid formations. (a) non-rigid formation (a deformed formation with dashed lines is shown); (b) minimally rigid formation; (c) rigid but non-minimally rigid formation. . . . . . . . . 13

2.2 Examples of a non-infinitesimally-rigid framework and an infinitesimally rigid framework. (a) A rigid but not infinitesimally rigid framework. The collinear positions of the three nodes lead to $\operatorname{rank}(R)=1<$ 3. The red arrow indicates a non-trivial infinitesimal motion. (b) An infinitesimally rigid framework. . . . . . . . . . . 13

3.1 An example of a pair of flip/flex formations. (a) A target formation defined by five inter-agent distances. (b) A flip version of the target formation with the same set of five desired distances. In rigid formation control, they are both considered as correct equilibria for a target formation specified by given desired distances. . . . . . . . . . . . 25

3.2 An example of a correct equilibrium and a degenerate equilibrium for a four-agent formation shape with five given distances. (a) The correct formation shape, in which all the five distances are achieved. (b) An example of collinear degenerate equilibrium in $\mathbb{R}^{2}$, in which all the four agents converge to a line formation. . . . . . . . . . . 26

3.3 The sets of collinear or coplanar positions are invariant for 2-D/3-D

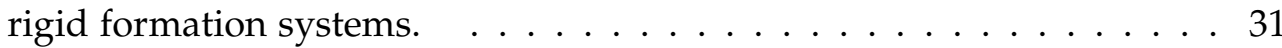

3.4 If all agents start at non-collinear (non-coplanar) positions, then their positions will be non-collinear (non-coplanar) at any finite or infinite time. . . . . . . . . . . . . . . . . . . . . 31

4.1 Several examples of controller functions $g_{k}$ studied in the literature (which correspond to the controller functions (4.5), (4.7), (4.9) and (4.10) discussed above). In all plots, we let $d_{k}=4$ and thus $e_{k} \in$ $(-16, \infty)$

4.2 Two novel controller functions $g_{k}$ with guaranteed exponential convergence of the distance error system. In both plots, we let $d_{k}=4$ and thus $e_{k} \in(-16, \infty)$. The left figure shows the proposed function in (4.29), and the right figure shows the proposed function in (4.31). . . . . 46 
4.3 An example of graph decomposition of a non-minimally rigid framework. The dashed lines in the decomposed subgraphs indicate the removed edges. . . . . . . . . . . . . . . . . 4 47

4.4 Simulation on stabilization control of an 4-agent rectangular formation shape. The initial and final positions are denoted by circles and squares, respectively. The initial formation is denoted by dotted blue lines, and the final formation is denoted by red solid lines. The black star denotes the formation centroid, which is stationary. . . . . . . . 50

4.5 Exponential convergence of the distance errors for 2-D rectangular for-

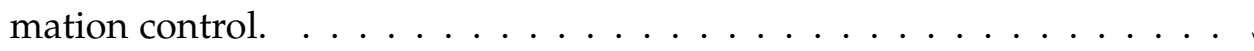

4.6 Simulation on stabilization control of a double tetrahedron formation in 3-D space. The initial and final positions are denoted by circles and squares, respectively. The initial formation is denoted by dashed lines, and the final formation is denoted by red solid lines. The black star denotes the formation centroid, which is stationary. . . . . . . . . 51

4.7 Exponential convergence of the distance errors for 3-D tetrahedron formation control. . . . . . . . . . . . . . . . . . . 51

5.1 An undirected rigid tetrahedron formation. . . . . . . . . . . . . . . . . . . . 58

5.2 Convergence of distance errors. . . . . . . . . . . . . . . . 67

5.3 Rigid motions (helical motions) induced by distance mismatches in a tetrahedron formation. . . . . . . . . . . . .

6.1 Example of controlling a 2-D rectangular formation and a 3-D tetrahedral formation with prescribed orientation. (a) Agent 1 and one of its neighbors, agent 2 , are chosen as orientation agents. The relative position vector $p_{2}-p_{1}$ associated with edge $(1,2)$ is used to describe the desired orientation, which is denoted by red color (in this example $\left.(1,2) \in \mathcal{E}_{o}\right)$. (b) Agent 1 and two of its neighbors, agents 2 and 4, are chosen as orientation agents. The relative position vectors $p_{2}-p_{1}$ and $p_{4}-p_{1}$ associated with edges $(1,2)$ and $(1,4)$ are used to describe the desired orientation, which are denoted by red color (in this example $\left.(1,2),(1,4) \in \mathcal{E}_{0}\right) \ldots \ldots \ldots \ldots \ldots \ldots \ldots \ldots$

6.2 Stabilization of a rigid rectangular formation without orientation control. The initial and final positions are denoted by circles and squares, respectively. The initial formation is denoted by dotted blue lines, and the final formation is denoted by red solid lines. The black star denotes the formation centroid.

6.3 Stabilization of a rigid rectangular formation with prescribed orientation. The initial and final positions are denoted by circles and squares, respectively. The initial formation is denoted by dotted blue lines, and the final formation is denoted by red solid lines. The black star denotes the formation centroid. 
6.4 Convergence of the distance/orentation errors with the proposed controller (6.2). The orientation error $\phi_{12}$ is defined as $\phi_{12}=\arctan \left(\left(p_{2, y}-\right.\right.$ $\left.\left.p_{1, y}\right) /\left(p_{2, x}-p_{1, x}\right)\right)-\pi / 2 . \ldots \ldots \ldots . \ldots \ldots 7$

6.5 Stabilization of a 3-D rigid formation with prescribed orientation. The initial and final positions are denoted by circles and squares, respectively. The initial formation is denoted by dotted blue lines, and the final formation is denoted by red solid lines. The black star denotes the formation centroid. . . . . . . . . . . . . . . . . 8 87

7.1 Logarithmic quantizer function with the gain $\delta_{u}=0.5$, defined in (7.3). 94

7.2 (a) Symmetric uniform quantizer function, defined in (7.2). (b) Asymmetric uniform quantizer function, defined in (7.23) . . . . . . . . . . . . 104

7.3 Stabilization control of a rigid formation: symmetric uniform quantization case. Left: the trajectories of five agents and the final formation shape. Right: Time evolutions of the distance errors. . . . . . . . . . . 106

7.4 Stabilization control of a rigid formation: logarithmic quantization case. Left: the trajectories of five agents and the final formation shape. Right: Time evolutions of the distance errors. . . . . . . . . . . . . . . 107

7.5 Stabilization control of a rigid formation: binary measurement case. Left: the trajectories of five agents and the final formation shape. Right: Time evolutions of the distance errors. . . . . . . . . . . . . . . 107

7.6 Stabilization control of a rigid formation: asymmetric uniform quantization case. Left: the trajectories of five agents and the final formation shape. Right: Time evolutions of the distance errors. . . . . . . . . . . 107

8.1 Simulation on shape stabilization control of a double tetrahedron formation in the 3-D space with double-integrator systems. . . . . . . . 133

8.2 Simulation on formation flocking control of a double tetrahedron formation in the 3-D space with double-integrator systems. . . . . . . . 134

8.3 Simulation on formation shape control of a double tetrahedron formation in the 3-D space with single-integrator systems. The initial conditions are chosen to be in a plane in the 3-D space, and the formation converges to an incorrect formation shape that lives in that plane (i.e. an incorrect planar equilibrium formation). . . . . . . . . . . . . . 135

8.4 Simulation on formation shape control of a double tetrahedron formation in the 3-D space with double-integrator systems. The formation converges to a correct formation shape, even if one chooses degenerate coplanar initial positions. . . . . . . . . . . . . . . . 135

9.1 Two feasible formations with a group of constant-speed agents. . . . . 146

9.2 Feasible motions for heterogeneous agent group. Type (i): $w_{1}=0$ and $w_{2}=0$; Type (ii): $w_{1}=1$ and $w_{2}=0$; Type (iii): $w_{1}=0$ and $w_{2}=1$; Type (iv): $w_{1}=1$ and $w_{2}=1$. The solid markers indicate the final positions for all the agents. . . . . . . . . . . . . . . . . 152 


\section{Introduction}

\subsection{Research background: cooperative control and networked systems}

The research problems to be discussed in this thesis fall into the broad scope of cooperative control and networked systems, which have gained considerable attention in the last decade in the control community. According to the recently published Encyclopedia of Systems and Control [Baillieul and Samad, 2015], networked systems can be loosely defined as "a system of systems", or "a collection of agents that interact with each other" ${ }^{1}$. The word agent can refer to autonomous robots, unmanned aerial vehicles (UAVs), or mobile sensors, depending on the different control context (see also [Ren and Cao, 2010]).

The central themes in the study of cooperative control for networked multi-agent systems include the understanding of the role of cooperation, the mechanism of information sharing between distributed agents, the stability and achievement of a global task arising from local interactions, the robustness against measurement/communication perturbations, and the fundamental limitations or tradeoffs between local tasks and global tasks, among others. In contrast to centralized control systems, distributed networked systems and cooperative control enjoy several nice advantages, including flexible scalability to the system size and change of agent number, strong adaptivity to the change of environment, and low operational cost without increasing the complexities of the whole system. Some excellent surveys for recent progress on distributed multi-agent coordination and networked systems can be found in [Cao et al., 2013; Zhang et al., 2013; Knorn et al., 2016].

We mainly consider coordination and formation control for multi-agent systems in this thesis. Formation control and shape stabilization is typically a distributed and cooperative control task, in that each agent uses only local information/measurements to achieve a cooperative formation task. Roughly speaking, formation control aims to design distributed controllers such that a group of spatially distributed agents could reach some pre-defined formations involving geometric relationships between them. Such geometric relationships can be described by relative positions, bearings, distances, or a mix of different geometric variables, depending on the con-

\footnotetext{
${ }^{1}$ See [Baillieul and Samad, 2015, Page 849]: the entry networked systems contributed by J. Cortés.
} 
text and control requirement. In this thesis, we will focus in particular on rigid formation shape control ${ }^{2}$, in which the formation shape is defined by a certain set of inter-agent distances.

\subsection{Introduction and literature review}

In this section, some relevant and recent papers for each research theme will be briefly reviewed. We remark that in the beginning of each main chapter, a more detailed literature review will be provided for each research topic.

\subsubsection{Different approaches on formation shape control}

Formation control for a group of autonomous mobile agents has attracted much research interest due to its broad applications in many areas including both civil and military fields. According to several surveys, most approaches on formation control in the literature can be classified as leader-follower strategy, virtual structure approach and behavior-based method (see e.g. the review in [Fahimi, 2008]). A key problem in formation control that receives particular interest is how to stabilize and maintain a geometrical formation shape in a distributed manner, and this will be one of the central focuses of this thesis. In the recent survey paper [Oh et al., 2015], different types of formation shape control strategies are reviewed and compared in terms of the sensing capability and the interaction topology, among which two most commonly-used approaches are

- The displacement-based approach, in which the desired formation is specified by a certain set of inter-agent relative positions and linear controllers turn out to be possible;

- The distance-based approach, in which the desired formation is specified by a certain set of inter-agent distances and nonlinear control laws are required.

There are several distinct properties for the above two approaches. For the first approach, all the agents must have their coordinate bases with the same orientation (while the origins may be different); this means that the desired relative position vectors can be well defined and controlled between agents (see e.g. [Ren and Beard, 2008; Xiao et al., 2009]). In turn, this means that all the agents should be equipped with a compass or equivalent to guarantee their coordinate orientation alignments, which may not be practical in real world applications since having consistency among the compass readings would be a challenging task due to instrument bias and/or local variations caused by, e.g., the earth's magnetic field. We will review the requirement of coordinate frame alignment in formation control in the following section, together with some other recently proposed formation control algorithms that were not reviewed in [Oh et al., 2015].

\footnotetext{
${ }^{2}$ Relevant concepts of graph rigidity theory will be provided in Chapter 2.
} 


\subsubsection{Coordinate frame requirement in formation control}

The coordinate frame requirement was largely ignored in early works on formation control (as reviewed in [Oh et al., 2015]). It is only in recent years that the importance of coordinate frame alignment has been recognized in formation controller design and implementation. It has also been shown in [Meng et al., 2016] that the assumption that all the agents have coordinate systems with the same orientation may not be realistic in practice, since small perturbations in their local coordinate systems will cause unexpected behaviors for the displacement-based formation system. Thus in practice, a coordinate-free formation control system is almost always preferable. In [Aranda et al., 2015], a coordinate-free formation control strategy was proposed by including a rotation matrix in the formation controller. The advantage of the coordinate-free property of the proposed formation controller in [Aranda et al., 2015] is offset by the requirement that the relative position measurements from all other agents should be available to each individual agent, which implies that the coordinate-free formation control in [Aranda et al., 2015] is effectively not a distributed one. A graph Laplacian-based approach has been proposed in [Lin et al., 2016b] which enables coordinate-free formation stabilization of target shapes (defined as an affine formation). Recent efforts also show that the bearing-based approach is another promising strategy to achieve a desired formation [Zhao and Zelazo, 2016] which is translation- and scaling-invariant. We note that such an approach however still does not resolve the strict requirement for global knowledge of coordinate frame orientation among all agents.

In the case that a common coordinate orientation is required for all the agents and initially they have different local coordinate frames, one needs to design a combined control establishing coordinate frame direction alignment and formation shape stabilization to ensure the convergence to a target shape. This idea has been developed and discussed in detail in e.g. [Cortés, 2009; Oh and Ahn, 2014b; Zhao and Zelazo, 2016; Montijano et al., 2016; Tron et al., 2016] in different control scenarios.

The requirement on the coordinate frame alignment can be avoided in the distancebased formation setup. This is because in the distance-based setup a global coordinate system defining a common orientation for all individual agents' coordinate frames is not required, and each agent can use its local coordinate basis to achieve a rigid formation shape. ${ }^{3}$ Partly because of this, the distance-based control approach attracts particular interest in the field of formation control.

\footnotetext{
${ }^{3}$ A very nice result on how to determine the coordinate-free property (termed $S E(N)$ invariance property) in networked control system has been established in [Vasile et al., 2015]. The main result in [Vasile et al., 2015] indicates that a pairwise interaction system is $S E(N)$ invariant if and only if it is quasilinear. We refer the readers to [Vasile et al., 2015] for more discussions on the $S E(N)$ invariance property. It can be proven that formation controllers derived from the distance-based control framework satisfy this quasi-linear property and thus the implementation of a distance-based formation control system is coordinate-free.
} 


\subsubsection{Equilibrium and stability analysis on rigid formation control sys- tems}

For distance-based formation control, graph rigidity theory [Asimow and Roth, 1979] plays a central role. The first paper that considered rigidity-based formation control is probably [Olfati-Saber and Murray, 2002], in which a graph theoretical framework was proposed to employ rigidity theory [Asimow and Roth, 1979] to define a target formation shape and to construct a formation potential. A comprehensive study of the equilibrium and stability for gradient-based rigid formation control system was performed in [Krick et al., 2009], in which the authors proved the local asymptotical stability of the equilibrium manifold by assuming that the target formation is infinitesimally rigid ${ }^{4}$.

Several recent papers including [Dimarogonas and Johansson, 2009; Oh and Ahn, 2014a; Cai and De Queiroz, 2015] have studied distance-based formation control for different formation shapes described by undirected graphs, mostly focusing on local convergence. Formation shape stabilization with communications and interactions modeled by directed graphs has been reported in [Dörfler and Francis, 2010; Cao et al., 2011; Summers et al., 2011] and [Yu et al., 2009].

Since the rigid formation control system involves nonlinear control terms, a complete analysis of the equilibrium sets and their stability property is very challenging. For this reason, some recent efforts have been devoted to understanding formation systems with a simple shape like triangular shape [Cao et al., 2011], tetrahedral shape [Park et al., 2014] and rectangular shape [Dasgupta et al., 2011]. In order to better understand (and estimate) the bounds of numbers of different types of equilibrium sets, Morse theory was applied in the study of rigid formation control systems [Anderson, 2011; Helmke and Anderson, 2013; Anderson and Helmke, 2014]. In later chapters, we will give a more comprehensive review on this topic.

\subsubsection{Some practical issues in rigid formation control}

In order to achieve a rigid target formation shape, each agent needs to acquire the real-time relative information (in terms of relative positions) from its neighboring agents, and should agree on what the global task is. In [Belabbas et al., 2012], a key robustness issue in rigid formation control systems was first identified, by considering that some neighboring agents may have different views on what the target distances between them should be, or they may have biases in their distance measurements. A more comprehensive investigation on this robustness issue for undirected 2-D rigid formations was reported in [Mou et al., 2016], which shows that the formation shape may converge to an approximated one close to the target formation shape, while additional circular motion would occur almost always due to mismatched distances. A further study on 3-D rigid formations via a tetrahedral formation as an example was shown in [Sun et al., 2013], which indicates that generically a helical motion will occur for 3 -D rigid formations with distance mismatches. In this the-

\footnotetext{
${ }^{4}$ The definition of infinitesimal rigidity will be given in Chapter 2.
} 
sis, we will further discuss this problem, and give a more complete report on the robustness issue for general 3-D rigid formations.

As mentioned earlier, in a rigid formation control problem, the aim is to stabilize and maintain a rigid target formation shape, while the orientation is not well defined and not controlled. To stabilize a target formation shape with a prescribed orientation, one may turn to the displacement-based formation control approach (as shown in [Oh et al., 2015]), or adopt other control approaches which may require all the agents to have knowledge of the global coordinate frame. We will devote one chapter in this thesis to addressing the control problem of stabilizing both formation shapes and orientations, and will show how minimal knowledge of a global coordinate orientation is required by a small subset of agents to achieve this control task.

Apart from the mismatches in distance measurements or perceived distances, another practical issue is on the effect of information inaccuracy in the measurement or actuation caused by the intrinsic property of sensors or actuators. Quantization is a typical case to cause such information inaccuracy. In recent years, the study of networked control systems with quantized measurements has been an active topic in the control community [Cao et al., 2013; You et al., 2015]. Formation control with quantized measurements has been discussed in e.g. [De Persis et al., 2010; Liu et al., 2014; Jafarian and De Persis, 2015], while a complete analysis of the quantization effects on rigid formation control system is still lacking. This motivates us to further investigate the dynamics and convergence property for rigid formation control systems with quantized measurements.

\subsubsection{Agent dynamical model: beyond single-integrator models}

As reviewed in [Cao et al., 2013; Oh et al., 2015; Knorn et al., 2016], most papers on formation control assume that the agents in a formation control system are modelled by single integrators. This assumption allows one to focus on the dynamics and convergence property at the formation control task level, however it may hinder the issue of design and convergence analysis when agents have their own (linear or nonlinear) system dynamics. Apart from single-integrator models, more realistic models that have also been adopted extensively in the literature include doubleintegrator models [Cao et al., 2013] particulary in linear consensus research, and unicycle models on rendezvous control of distributed robotics [Francis and Maggiore, 2016]. Other models on formation control have also been reported in the literature; see e.g. [Abdessameud and Tayebi, 2013] on VTOL (vertical take-off and landing) models for attitude synchronization and formation control of a group of UAVs, and [Dong, 2015] for higher-order linear models on formation and containment control.

Double-integrator models have been used extensively for flocking control of multiagent systems in recent years, partly triggered by the pioneering works [Olfati-Saber, 2006] and [Tanner et al., 2007]. For rigid formation control systems modelled by double integrators, the results only appear sparsely in the literature, with some recent investigations in e.g. [Oh and Ahn, 2014a; Zhang et al., 2015; Deghat et al., 2016]. 
This motivates us to consider the double-integrator rigid formation control system in full detail, which will be reported in a later chapter in this thesis.

A fundamental problem in cooperative control is to understand the effect of each agent's local dynamic constraint on the global task achievement. A concept termed motion feasibility was developed in [Tabuada et al., 2005], in which an elegant criterion was proposed to guarantee the existence of feasible trajectories for all the agents under both formation constraints and kinematics constraints. The agent's model used in [Tabuada et al., 2005] is a drift-free control system, which however cannot encompass some commonly-used agent models in this framework. For example, a particular constraint in the cooperative formation control design is that on occasions UAVs used in the control task (e.g. Aerosonde UAVs or other types of fixed-wing aircraft) usually fly most efficiently at fixed, nominal speeds [Anderson et al., 2008a]. The control problem of coordinating constant-speed agents is a research problem posed to us by the Australian Defence Science and Technology Group (DSTG) on the practical use of such UAVs. To this purpose, agent's dynamics described by affine nonlinear control systems with possible drifts [Isidori, 1995] are more reasonable and will provide more insight to understand the interplay between individual agent's kinematic constraints and formation task constraints. Based on this, we will devote a chapter in this thesis to developing a formation feasibility theory and motion generation technique in this scenario, which aims to address a fundamental problem in the field of coordination control of multi-agent systems.

\subsection{Thesis outline and statements of collaborations}

This thesis consists of ten chapters, including the current chapter, the next chapter on theoretical preliminaries, followed by seven main chapters showing main technical results, and the last chapter providing conclusions. The following is a brief outline of the thesis structure as well as the content in each chapter. According to the different emphases of research topics and proposed research problems, the seven main chapters (Chapters 3-9) are divided into three parts as shown in the list.

Also, after the introduction of each main chapter (Chapters 3-9), a brief summary of the nature of the collaboration is reported when appropriate. The author is primarily responsible for the contribution of research outcomes in each main chapter.

1. Chapter 1 presents a general introduction to the research background, state-ofart results in the research field, as well as research problems to be discussed in this thesis.

2. Chapter 2 provides certain theoretical preliminaries on graph theory, rigidity theory, gradient systems that will be frequently applied in the discussions of technical results in later chapters.

Part I: Formation control systems: equilibrium and stability analysis

This part consists of Chapter 3 and Chapter 4. 
3. Chapter 3 focuses on equilibrium analysis of the standard rigid formation control system, and establishes a rank-invariant property which provides more insights on the stability and dynamics property of such systems.

This is joint work with U. Helmke (University of Würzburg, Germany), and B. D. O. Anderson. Part of the research in this chapter was preformed during a research visit at University of Würzburg, Germany.

4. Chapter 4 aims to establish the exponential stability of the distance error system (definitions will become clear in the context) arising in rigid formation control. The results in this section will be theoretical basis for the robustness analysis for rigid formation control systems in the presence of mismatched distances, as discussed in Chapter 5.

This is joint work with B. D. O. Anderson, S. Mou (Purdue University, USA) and M. Cao (University of Groningen, The Netherlands).

Part II: Formation control systems: some practical considerations

This part consists of Chapter 5, Chapter 6 and Chapter 7.

5. In Chapter 5, we discuss the robustness issues in 3-D rigid formation control systems ${ }^{5}$ with mismatched distances, investigate the rigid motion property induced by distance mismatches, and explore how such mismatches can be used to steer a rigid formation.

This is joint work with A. S. Morse (Yale University), B. D. O. Anderson, S. Mou (Purdue University, USA). Part of the research in this chapter was done during a research visit at Yale University.

6. Chapter 6 discusses the stabilization control problem of rigid formations with desired shapes and prescribed orientations. The main contributions are a description of the formation controller, the proof of the minimum number of agents with knowledge of global coordinate orientation, and the stability analysis.

This work is a collaboration of B. D. O. Anderson, M.-C. Park and H.-S. Ahn from Gwangju Institute of Science and Technology (GIST), South Korea. Part of the research in this chapter was preformed during a research visit to GIST.

7. Chapter 7 explores the quantization effect on rigid formation control, motivated by the fact that many distributed control systems are equipped with digital sensors and actuators which only produce quantized measurements or feedbacks.

This work is a collaboration with H. Garcia de Marina (École nationale de l'aviation civile, France), B. D. O. Anderson, and M. Cao (University of Groningen, The Netherlands). Part of the research in this chapter was finished during a research visit at University of Groningen.

\footnotetext{
${ }^{5}$ The robustness issues for 2-D rigid formation control systems are discussed in a joint paper [Mou et al., 2016].
} 


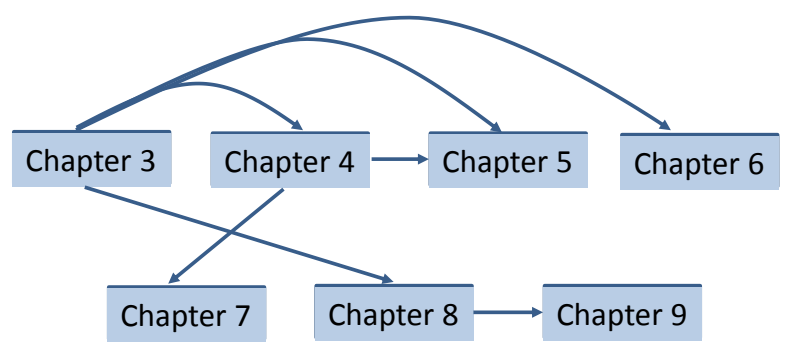

Figure 1.1: An overview of relations between the main chapters.

\section{Part III: Distributed coordination control: general system models} This part consists of Chapter 8 and Chapter 9.

8. Chapter 8 extends several results on equilibrium analysis of single-integrator formation systems discussed in previous chapters (in particular in Chapter 3) to rigid formation systems modelled by double integrators. To be specific, we consider two types of double-integrator formation systems in this chapter, namely, formation stabilization systems and flocking control systems. Some invariance principles and links between single-integrator formation systems and double-integrator formation systems will be established, such that the results in previous chapters on single-integrator systems can be applied to the study of double-integrator systems.

The results in this chapter are from joint work with B. D. O. Anderson, M. Deghat (University of New South Wales) and H.-S. Ahn (GIST, South Korea).

9. Chapter 9 considers a very general yet fundamental problem in multi-agent coordination control, namely the formation feasibility of distributed control in the presence of both formation constraints from the global task level, and kinematic constraints from each agent. The agents' dynamics are modelled by affine control systems with possible drift terms, which include single-integrator models and double-integrator models as special cases. Some control problems in previous chapters can also fit in this general framework. We derive an algebraic condition to guarantee the existence of feasible formations for such group of agents, and propose a systematic way to generate feasible trajectories for a group of heterogeneous agents.

The results are from close collaboration and extensive discussions with B. D. O. Anderson. We also appreciate insightful discussions with S. Drake (Australian Defence Science and Technology Group) on this problem, and his feedbacks on how this control framework can be used in the coordination control of multiple constant-speed UAVs.

10. Chapter 10 presents a short summary of the main results and contributions of this thesis, and also indicates some possible directions for future research.

In addition to the above structure outline, an overview of relations between each main chapter is shown in Figure 1.1. 


\section{Theoretical preliminaries}

This chapter introduces several essential concepts and theoretical foundations on graph theory, rigidity theory and gradient systems that will be frequently applied in later chapters. We also review gradient descent formation control for stabilization of rigid formation shapes [Krick et al., 2009] in the last section of this chapter, which will be revisited in several chapters in this thesis.

\subsection{Notations}

The notations used in this thesis are fairly standard. A real valued function $f$ is called a $C^{r}$ function if it has continuous first $r$ derivatives. We use $\mathbb{R}^{n}$ to denote the $n$-dimensional Euclidean space, and $\mathbb{R}^{m \times n}$ to denote the set of $m \times n$ real matrices. The transpose of a matrix or vector $M$ is denoted by $M^{\top}$. The rank, determinant, image (i.e., column space) and null space of a matrix $M$ are denoted by $\operatorname{rank}(M)$, $\operatorname{det}(M), \operatorname{Im}(M)$ and $\operatorname{null}(M)$, respectively. For a vector $v$, the symbol $\|v\|$ denotes its Euclidean norm. We use $\operatorname{span}\left\{v_{1}, v_{2}, \cdots, v_{k}\right\}$ to represent the subspace spanned by a set of $k$ vectors $v_{1}, v_{2}, \cdots, v_{k}$. We denote the $n \times n$ identity matrix and zero matrix as $I_{n \times n}$ and $\mathbf{0}_{n \times n}$, respectively. Let $\mathbf{1}_{n}$ and $\mathbf{0}_{n}$ be the $n$-dimensional vectors of all ones and all zeros. When the subscripts for $I_{n \times n}, \mathbf{0}_{n \times n}, \mathbf{1}_{n}$ or $\mathbf{0}_{n}$ are omitted, their dimensions should be clear in the context.

The inertia of a matrix $M \in \mathbb{R}^{n \times n}$ is given by the triple $\left\{v_{+}, v_{-}, v_{0}\right\}$, where $v_{+}$ (respectively, $v_{-}$) denotes the number of unstable (respectively, stable) eigenvalues of $M$ in the open right (respectively, left) complex half plane, and $v_{0}$ denotes the number of eigenvalues with zero real part (see e.g. [Carlson and Schneider, 1963]). The symbol $\otimes$ denotes Kronecker product. $S E(d)$ and $S O(d)$ denote the special Euclidean group and the special orthogonal group, respectively.

Note in later chapters we may use additional notations that will be solely used in that particular chapter. This will be made clear in the corresponding chapter. 


\subsection{Preliminary on graph theory}

Consider an undirected graph with $m$ edges and $n$ vertices (or nodes), denoted by $\mathcal{G}=(\mathcal{V}, \mathcal{E})$ with vertex set $\mathcal{V}=\{1,2, \cdots, n\}$ and edge set $\mathcal{E} \subset \mathcal{V} \times \mathcal{V}$. The neighbor set $\mathcal{N}_{i}$ of node $i$ is defined as $\mathcal{N}_{i}:=\{j \in \mathcal{V}:(i, j) \in \mathcal{E}\}$. The matrix relating the nodes to the edges is called the incidence matrix $H=\left\{h_{i j}\right\} \in \mathbb{R}^{m \times n}$, whose entries are defined as (with arbitrary edge orientations)

$$
h_{i j}=\left\{\begin{array}{cc}
1, & \text { the } i \text {-th edge sinks at node } j ; \\
-1, & \text { the } i \text {-th edge leaves node } j ; \\
0, & \text { otherwise. }
\end{array}\right.
$$

Another important matrix representation of a graph $\mathcal{G}$ is the Laplacian matrix $L(\mathcal{G})$ [Mesbahi and Egerstedt, 2010]. For an undirected graph, the associated Laplacian matrix can be written as $L(\mathcal{G})=H^{\top} H$. For a connected undirected graph, one has $\operatorname{rank}(L)=n-1$ and $\operatorname{null}(L)=\operatorname{null}(H)=\operatorname{span}\left\{\mathbf{1}_{n}\right\}$. For more introductions on algebraic graph theory and their applications in distributed and networked control, we refer the readers to [Bapat, 2010] and [Mesbahi and Egerstedt, 2010].

\subsection{Preliminary on rigidity theory}

\subsubsection{Definitions and properties}

Let $p_{i} \in \mathbb{R}^{d}$ denote a point that is assigned to $i \in \mathcal{V}$ in the $d$-dimensional Euclidean space $\mathbb{R}^{d} .{ }^{1}$ The stacked vector $p=\left[p_{1}^{\top}, p_{2}^{\top}, \cdots, p_{n}^{\top}\right]^{\top} \in \mathbb{R}^{d n}$ represents the realization of $\mathcal{G}$ in $\mathbb{R}^{d}$. The pair $(\mathcal{G}, p)$ is said to be a framework (or a formation, in our context of formation control) of $\mathcal{G}$ in $\mathbb{R}^{d}$. By introducing the matrix $\bar{H}:=H \otimes I_{d} \in \mathbb{R}^{d m \times d n}$, one can construct the relative position vector as an image of $\bar{H}$ from the position vector $p$ :

$$
z=\bar{H} p
$$

where $z=\left[z_{1}^{\top}, z_{2}^{\top}, \cdots, z_{m}^{\top}\right]^{\top} \in \mathbb{R}^{d m}$, with $z_{k} \in \mathbb{R}^{d}$ being the relative position vector for the vertex pair defined by the $k$-th edge.

Consistent with the assumed ordering of the edges in $\mathcal{E}$, the distance function $r_{\mathcal{G}}(p): \mathbb{R}^{d n} \rightarrow \mathbb{R}^{m}$ associated with the framework $(\mathcal{G}, p)$ is given as:

$$
r_{\mathcal{G}}(p)=\frac{1}{2}\left[\cdots,\left\|p_{i}-p_{j}\right\|^{2}, \cdots\right]^{\top},(i, j) \in \mathcal{E}
$$

in which the $k$-th component in $r_{\mathcal{G}}(p), \frac{1}{2}\left\|p_{i}-p_{j}\right\|^{2}$, corresponds to half of the squared length of the relative position vector $z_{k}$ which connects the vertices $i$ and $j$. We first show the definitions of congruence and equivalence of two frameworks $(\mathcal{G}, p)$ and $(\mathcal{G}, q)$ (see e.g. [Asimow and Roth, 1978], [Eren et al., 2003]).

\footnotetext{
${ }^{1}$ In this thesis we will mostly consider $d \in\{2,3\}$. Some results obtained in the following chapters can also be extended to arbitrary dimensional spaces. This will be made clear in the context.
} 
Definition 1. $(\mathcal{G}, p)$ and $(\mathcal{G}, q)$ are equivalent if they have the same edge lengths, i.e., $\| p_{i}-$ $p_{j}\|=\| q_{i}-q_{j} \|, \forall(i, j) \in \mathcal{E}$.

Definition 2. $(\mathcal{G}, p)$ and $(\mathcal{G}, q)$ are congruent if $\left\|p_{i}-p_{j}\right\|=\left\|q_{i}-q_{j}\right\|, \forall i, j \in \mathcal{V}$.

We refer the readers to [Connelly and Guest, 2015] for more discussions and examples on framework equivalence and framework congruence. The rigidity of frameworks is then defined as follows.

Definition 3. ([Asimow and Roth, 1979]) A framework $(\mathcal{G}, p)$ is rigid in $\mathbb{R}^{d}$ if there exists a neighborhood $\mathbb{U}$ of $p$ such that $r_{\mathcal{G}}^{-1}\left(r_{\mathcal{G}}(p)\right) \cap \mathbb{U}=r_{\mathcal{K}}^{-1}\left(r_{\mathcal{K}}(p)\right) \cap \mathbb{U}$ where $\mathcal{K}$ is the complete graph with the same vertices as $\mathcal{G}$.

The above definition may seem to be too technical, but there is an intuitive interpretation by using concepts from joints and bars in a structure. The notion of rigidity then corresponds to the undeformability of a structure of joints and bars [Hendrickson, 1992]. In other words, when the only allowed smooth motions are those that preserve the distance between every pair of joints, the framework is said to be rigid. Otherwise it is flexible. Figure 2.1 shows several examples of two-dimensional rigid and non-rigid formations. The definition of minimal rigidity that is also shown in Figure 2.1 will be given in the next section.

\subsubsection{Rigidity matrix and related results}

In the following, the set of all frameworks $(\mathcal{G}, p)$ which satisfies the distance constraints is referred to as the target formation. One useful tool to characterize the rigidity property of a framework is the rigidity matrix $R \in \mathbb{R}^{m \times d n}$, which is defined as

$$
R(p)=\frac{\partial r_{\mathcal{G}}(p)}{\partial p}
$$

It is not difficult to see that each row of the rigidity matrix $R$ takes the following form

$$
\left[\mathbf{0}_{1 \times d}, \cdots,\left(p_{i}-p_{j}\right)^{\top}, \cdots, \mathbf{0}_{1 \times d}, \cdots,\left(p_{j}-p_{i}\right)^{\top}, \cdots, \mathbf{0}_{1 \times d}\right]
$$

Each edge gives rise to a row of $R$, and, if an edge links vertices $i$ and $j$, then the nonzero entries of the corresponding row of $R$ are in the columns from $d i-(d-1)$ to $d i$ and from $d j-(d-1)$ to $d j$.

We indicate a simple expression for the rigidity matrix which involves both the network topology and position configuration. Recall (2.1), which shows that the relative position vector lies in the image of $\bar{H}$. The distance function is a map from the node positions to the squared edge lengths. Thus we can redefine the distance function, $g_{\mathcal{G}}(z): \operatorname{Im}(\bar{H}) \rightarrow \mathbb{R}^{m}$ as $g_{\mathcal{G}}(z)=\frac{1}{2}\left[\left\|z_{1}\right\|^{2},\left\|z_{2}\right\|^{2}, \cdots,\left\|z_{m}\right\|^{2}\right]^{\top}$. From (2.1) 
and (2.3), one can obtain the following simple form for the rigidity matrix

$$
\begin{aligned}
R(p)=\frac{\partial r_{\mathcal{G}}(p)}{\partial p} & =\frac{\partial g_{\mathcal{G}}(z)}{\partial z} \frac{\partial z}{\partial p} \\
& =\left(\begin{array}{ccc}
z_{1}^{\top} & \cdots & 0 \\
\vdots & \ddots & \vdots \\
0 & \cdots & z_{m}^{\top}
\end{array}\right) \bar{H} \\
& =Z^{\top} \bar{H}
\end{aligned}
$$

where $Z=\operatorname{diag}\left\{z_{1}, z_{2}, \cdots, z_{m}\right\} \in \mathbb{R}^{d m \times m}$. It is obvious that the entries of $R(p)$ are also functions of $z$, and we will also write it as $R(z)$.

Now we introduce an important concept in graph rigidity theory called infinitesimal rigidity. In order to understand infinitesimal rigidity, we first introduce the notion of infinitesimal motion. Consider the position of each vertex as a differentiable function of $t$ (which can be the time) and suppose $\left\|p_{i}(t)-p_{j}(t)\right\|^{2}=$ constant for every $(i, j) \in \mathcal{E}$. By differentiating the edge length constraints one can obtain $\left(v_{i}-v_{j}\right)^{\top}\left(p_{i}-p_{j}\right)=0$, where $v_{i}$ is the instantaneous velocity of vertex $i$. An assignment of velocities that satisfies such an equation for a particular framework is called an infinitesimal motion of that framework [Hendrickson, 1992]. The definition of infinitesimal rigidity for a framework is given as follows (see e.g., [Hendrickson, 1992; Hendrickx, 2008]).

Definition 4. A framework is infinitesimally rigid if all its infinitesimal motions are special Euclidean motions.

The rigidity matrix is useful to determine the infinitesimal rigidity of a framework, as shown in the following theorem.

Theorem 1. ([Hendrickson, 1992]) Consider a framework $(\mathcal{G}, p)$ in the d-dimensional space with $n \geq d$ vertices and $m$ edges. It is infinitesimally rigid if and only if

$$
\operatorname{rank}(R(p))=d n-d(d+1) / 2
$$

Specifically, under the condition that $n \geq d$ as shown in Theorem 1 , the framework $(\mathcal{G}, p)$ is infinitesimally rigid in $\mathbb{R}^{2}\left(\right.$ resp. $\left.\mathbb{R}^{3}\right)$ if and only if $\operatorname{rank}(R(p))=2 n-3$ (resp. $\operatorname{rank}(R(p))=3 n-6)$. Obviously, in order to have an infinitesimally rigid framework, the graph should have at least $2 n-3$ (resp. $3 n-6$ ) edges in $\mathbb{R}^{2}$ (resp. $\mathbb{R}^{3}$ ). If the framework is infinitesimally rigid in $\mathbb{R}^{2}$ (resp. $\mathbb{R}^{3}$ ) and has exactly $2 n-3$ (resp. $3 n-6)$ edges, then it is called a minimally and infinitesimally rigid framework.

In the examples shown in Figure 2.1, two of which are rigid and one of which is non-rigid. In a non-rigid formation, part of the formation can flex or move, while the rest of the formation remains stationary, and all distance constraints remain satisfied. Examples of a non-infinitesimally-rigid framework and an infinitesimally rigid framework are shown in Figure 2.2.

From the definition of infinitesimal rigidity, the following result is obvious. 

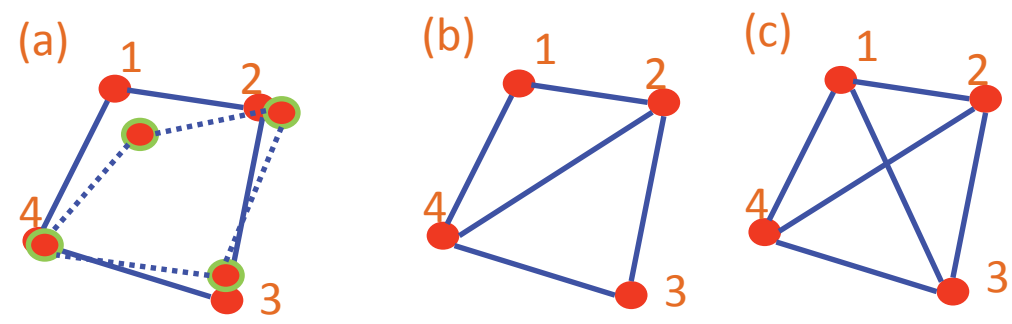

Figure 2.1: Examples of rigid and non-rigid formations. (a) non-rigid formation (a deformed formation with dashed lines is shown); (b) minimally rigid formation; (c) rigid but non-minimally rigid formation.
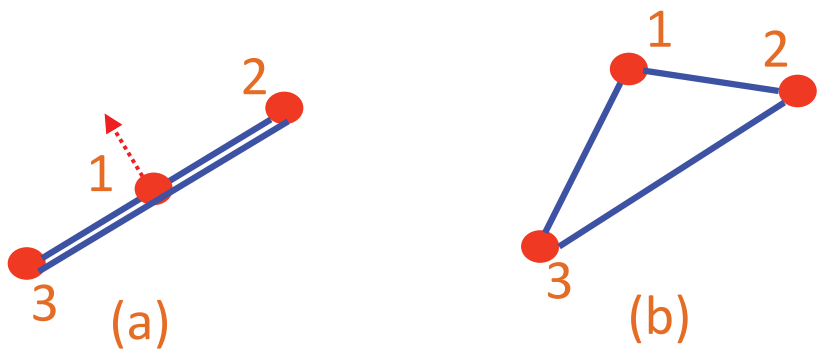

(b)

Figure 2.2: Examples of a non-infinitesimally-rigid framework and an infinitesimally rigid framework. (a) A rigid but not infinitesimally rigid framework. The collinear positions of the three nodes lead to $\operatorname{rank}(R)=1<3$. The red arrow indicates a non-trivial infinitesimal motion. (b) An infinitesimally rigid framework. 
Lemma 1. If the framework $(\mathcal{G}, p)$ is minimally and infinitesimally rigid in the d-dimensional space, then the matrix $R(z(p)) R(z(p))^{\top}$ is positive definite.

The following result will be useful for later analysis, which shows that there exists a smooth function which maps the distance set of the minimally rigid framework to the distance set of its corresponding framework modeled by a complete graph.

Lemma 2. Let $r_{\mathcal{G}}(q)$ be the distance function for a given infinitesimally minimally rigid framework $(\mathcal{G}, q)$ with agents'position vector $q$. Further let $\hat{r}_{\hat{\mathcal{G}}}(q)$ denote the distance function for an associated framework $(\hat{\mathcal{G}}, q)$, in which the vertex set remains the same as $(\mathcal{G}, q)$ but the underlying graph is a complete one (i.e. there exist $n(n-1) / 2$ edges which link all vertices). Then there exists a continuously differentiable function $f: r_{\mathcal{G}}(q) \rightarrow \mathbb{R}^{n(n-1) / 2}$ for which $\hat{r}_{\mathcal{G}}(q)=f\left(r_{\mathcal{G}}(q)\right)$ holds locally.

The proof of Lemma 2 is omitted here and can be found in [Mou et al., 2016]. In later chapters, we will frequently revisit Lemma 2 and apply it in the stability analysis for formation control systems with different properties.

We also refer to the paper [Anderson et al., 2008b] and the thesis [Hendrickx, 2008] for a detailed survey on using graph rigidity theory in modelling autonomous formations, and the extensions of graph rigidity theory for undirected graphs to graph persistence theory for directed graphs.

\subsection{Gradient systems}

\subsubsection{Definitions and properties}

In this section we briefly review the definition and properties of gradient systems. Let $V: \mathbb{R}^{n} \rightarrow \mathbb{R}_{\geq 0}$ be a scalar valued function that is $C^{r}$ with $r \geq 2$. Consider the following continuous-time system

$$
\dot{x}=-\nabla V(x)
$$

The above system is usually called a gradient system, and the corresponding function $V(x)$ is referred to as a potential function.

Gradient system enjoys several convergence properties due to the special structure of the gradient vector field in the right-hand side. Firstly, it should be clear that equilibrium points of (2.8) are critical points of $V(x)$. Moreover, at any point except for an equilibrium point, the vector field (2.8) is perpendicular to the level sets of $V(x)$. In fact, it is obvious to observe that $\dot{V}(x)=\nabla V(x)^{\top} \dot{x}=-\|\nabla V(x)\|^{2} \leq 0$, which indicates that the potential $V(x)$ is always nonincreasing along the trajectory of (2.8). The following results are also obvious.

Lemma 3. Consider the gradient system (2.8) with the associated potential $V(x)$.

- $\dot{V}(x) \leq 0$ and $\dot{V}(x)=0$ if and only if $x$ is an equilibrium point of (2.8). 
- Suppose $\bar{x}$ is an isolated minimum of a real analytic $V(x)$, i.e., there is a neighborhood of $\bar{x}$ that contains no other minima of $V(x)$. Then $\bar{x}$ is an asymptotically stable equilibrium point of (2.8).

The proof of the above lemma can be found in e.g. [Wiggins, 2003, Chapter 15]. Note that in the second statement we have emphasized the condition isolated minimum in the convergence property. As we will see in later chapters, for most formation control systems, their equilibrium points are not isolated but are continuum equilibria that form equilibrium orbits, which complicates the stability analysis. These will be made clear in the context.

We also refer the readers to the book [Wiggins, 2003, Chapter 15] and the thesis [Lageman, 2007] for more introductions and properties on gradient vector fields and gradient systems.

\subsubsection{Real analyticity and local minimum}

Note that a local minimum of $V$ is not necessarily a stable equilibrium point of (2.8), unless some more properties on the potential $V$ are imposed (while the smoothness of the potential $V$ is not enough). In [Absil and Kurdyka, 2006], several examples (and counterexamples) are carefully constructed to show the relationship between local minima of $V$ and stable equilibrium points of (2.8). In particular, it is shown in [Absil and Kurdyka, 2006] that with the analyticity ${ }^{2}$ of the potential $V$, local minimality becomes a necessary and sufficient condition for stability.

Theorem 2. ([Absil and Kurdyka, 2006]) Let $V$ be real analytic in a neighborhood of an equilibrium $\bar{x} \in \mathbb{R}^{n}$. Then, $\bar{x}$ is a stable equilibrium point of (2.8) if and only if it is a local minimum of $V$.

\subsection{Gradient descent control law for rigid formation stabi- lization}

The control goal in formation shape stabilization is to drive all the agents to reach a configuration such that a certain set of inter-agent distances can be achieved. We denote the target distance between agent $i$ and its neighboring agent $j$ as $d_{k_{i j}}$, for which the agent pair $i$ and $j$ should work cooperatively to achieve. The typical structure of the formation potential function is defined by

$$
V\left(p_{1}, \cdots, p_{n}\right)=\frac{1}{4} \sum_{\{i, j\} \in \mathcal{E}}\left(\left\|p_{i}-p_{j}\right\|^{2}-d_{k_{i j}}^{2}\right)^{2}
$$

It is obvious that the value of the above potential function for a formation is invariant if the given formation undergoes translation, rotation or reflection. By assuming

\footnotetext{
${ }^{2} \mathrm{~A}$ real function is analytic if it possesses derivatives of all orders and agrees with its Taylor series in the neighborhood of every point.
} 
that each agent's dynamics are described by a simple kinematic model in the form $\dot{p}_{i}=u_{i}, i \in\{1,2, \cdots, n\}$, where $u_{i} \in \mathbb{R}^{d}$ is the control input for agent $i$, an extensively studied formation control system is described by the following gradient descent control law

$$
\dot{p}_{i}=u_{i}=-\nabla_{p_{i}} V(p)=\sum_{j \in \mathcal{N}_{i}}\left(p_{j}-p_{i}\right)\left(\left\|p_{j}-p_{i}\right\|^{2}-d_{k_{i j}}^{2}\right)
$$

Note that in implementing the above control, each agent needs to measure the relative positions, denoted by $z_{k_{i j}}:=\left(p_{j}-p_{i}\right)$. We further define

$$
e_{k_{i j}}=\left\|p_{i}-p_{j}\right\|^{2}-\left(d_{k_{i j}}\right)^{2}
$$

to denote the squared distance error for edge $k$. In order to simplify the notation, we may use $e_{k}$ and $e_{k_{i j}}$ interchangeably in this thesis. This will also apply to $d_{k}$ and $d_{k_{i j}} z_{k}$ and $z_{k_{i j}}$ in later chapters when the dropping out of the dummy subscript $i j$ in each vector causes no confusion. The squared distance error vector is denoted by $e=\left[e_{1}, e_{2}, \cdots, e_{m}\right]^{\top}$.

This potential function (2.9) for rigid shape stabilization and the associated gradient flow (2.10) have been extensively studied in the literature; see e.g. [Krick et al., 2009; Anderson and Helmke, 2014; Cortés, 2009; Dörfler and Francis, 2010; Dimarogonas and Johansson, 2010; Cao et al., 2011; Anderson, 2011; Oh and Ahn, 2014a].

In several later chapters of this thesis, we will revisit the above formation control system (2.10), and investigate its convergence property as well as its variations. 


\section{Part I}

\section{Formation Control Systems: \\ Equilibrium and Stability Analysis}





\section{Invariance principles and equilibrium analysis for formation shape control systems}

\section{Chapter summary}

This chapter establishes a rank-invariance principle of the formation shape stabilization systems, which provides additional insights to the equilibrium analysis for such control systems. We further show some properties of the degenerate critical formations that live in a lower dimensional space, and prove that they are unstable. The implication of these results is that if all the agents start with generic initial positions, then their trajectories will be strictly bounded away from the set of degenerate formations at any finite or infinite time. These results are obtained from a joint analysis of rank-preserving flow theory, graph rigidity theory and invariant manifold theory.

\subsection{Introduction}

\subsubsection{Background and related work}

In the distance-based formation control framework, the global stability analysis beyond a local convergence for formation control systems with general shapes (other than the simple shapes such as triangular shapes or tetrahedral shapes) has been an open problem ([Krick et al., 2009; Anderson and Helmke, 2014]). The stability analysis of a formation control system usually involves an interplay of graph topology concepts and nonlinear analysis tools. On the one hand, there have been several papers focusing on the role of graph topology in formation control systems. In [Dimarogonas and Johansson, 2010] the authors discussed the convergence property for the distance-based formation control system when the underlying graph is a tree, and showed that with this assumption the commonly-used gradient descent control derived from a potential function can stabilize the formation system to the desired relative distances. However in this tree graph case the formation is not a rigid one and thus the shape is not well defined. Recently a vast amount of literature has 
emerged for analyzing formation control systems with certain simple and specific shapes, such as the 2-D triangular formation [Cao et al., 2011; Dörfler and Francis, 2010], the 2-D four-agent rectangular formation [Anderson et al., 2010] and the 3-D tetrahedral formation [Park et al., 2014]. For formation control systems with general shapes, however, a full understanding of the convergence properties for different equilibria has not yet been explored.

On the other hand, some other approaches have been reported recently to gain a further understanding of the bounds and properties of critical points of nonlinear formation control systems, including the semidefinite programming viewpoints for solving semialgebraic problems in [Summers et al., 2013] and Morse theory methods [Anderson, 2011; Helmke and Anderson, 2013; Anderson and Helmke, 2014]. The application of Morse theory to robot navigation control can be traced back to an early paper [Koditschek and Rimon, 1990]. It was recently shown in [Anderson, 2011] by using this theory that multiple equilibria, including incorrect equilibria, are a consequence of any formation shape control algorithm which evolves in a steepest descent direction using a smooth cost function that is invariant under translations and rotations. The existence of multiple equilibria of the potential function adds considerable complexity to the convergence analysis of formation control systems. We refer the readers to the recent thesis [Chen, 2014] for more discussions on applying Morse theory to the rigid formation control problem.

In this chapter, we aim to explore more invariance properties in formation control system and to provide additional insights on the stability analysis of critical formations. We will show a rank-preserving property for formation systems described by a gradient flow, which means that the dimension of the smallest affine space that contains all agents' positions is an invariant under the gradient flow. Furthermore, this rank-preserving property also indicates certain invariance properties for 2-D and 3-D formation systems.

In the second main part of this chapter, we will show that any incorrect equilibria which lie in a degenerate space (definitions will become clear later) are unstable. This is a consequence of the rank-preserving property of the formation gradient system and an eigenvalue result relating to the Hessian matrix of the potential function. The results hold for formation systems in any higher dimensions, which also have applications in other fields such as multidimensional scaling studies [Borg and Groenen, 2005]. Actually, the optimization problem involving minimizing certain stress functions in multidimensional scaling has a similar problem description as in the formation control field with similar forms of cost functions.

\subsubsection{Chapter organization}

The rest of this chapter is organized as follows. Section 3.2 presents formation equations and the problem setup. The rank-preserving property for formation systems in arbitrary dimensions is proved in Section 3.3. In Section 3.4, we discuss the stability property of degenerate equilibria (definitions will be clear in that section) and show they are unstable. Conclusions are provided in Section 3.5, which closes this chapter. 
In the Appendix 3.6, we briefly review some background on rank-preserving flow theory.

\subsection{Problem setup and motion equations}

We consider the standard formation control system for stabilizing a rigid target formation shape, as reviewed in Section 2.5 in Chapter 2. For the convenience of analysis we rewrite the formation control system as follows

$$
\dot{p}_{i}=-\sum_{j \in \mathcal{N}_{i}}\left(\left\|p_{i}-p_{j}\right\|^{2}-d_{k_{i j}}^{2}\right)\left(p_{i}-p_{j}\right), \quad i=1, \ldots, n
$$

which defines the steepest descent gradient flow of the potential function

$$
V(p)=\frac{1}{4} \sum_{(i, j) \in \mathcal{E}}\left(\left\|p_{i}-p_{j}\right\|^{2}-d_{k_{i j}}^{2}\right)^{2}
$$

In this chapter, we will revisit this formation control system, and establish several novel results involving a rank-invariance principle ${ }^{1}$ and their applications in the equilibrium analysis.

\subsection{Rank-preserving property for formation systems}

This section aims to show a rank-preserving property of the gradient system (3.1). The results hold for formation systems in general dimensions, and we will later specialize them to $d \in\{2,3\}$ cases.

By the definition of the incidence matrix $H$ and the rigidity matrix $R$, we can write the position system (3.1) in a compact form

$$
\dot{p}(t)=-R^{\top}(z) e=-\left(H^{\top} \otimes I_{d \times d}\right) Z(z) e
$$

Then the dynamics for the relative position vector $z$ can be written as

$$
\begin{aligned}
\dot{z}(t) & =\left(H \otimes I_{d \times d}\right) \dot{p}=-\left(H \otimes I_{d \times d}\right) R^{\top} e \\
& =-\left(\left(H H^{\top}\right) \otimes I_{d \times d}\right) Z(z) e
\end{aligned}
$$

\subsubsection{Proof of the rank-preserving property}

In this section we aim to prove the following rank-preserving property of the formation system (3.3).

Theorem 3. Denote $P:=\left[p_{1}, p_{2}, \cdots, p_{n}\right] \in \mathbb{R}^{d \times n}$ with the $i$-th column being agent $i$ 's position vector $p_{i}$, and denote $Q:=\left[z_{1}, z_{2}, \cdots, z_{m}\right] \in \mathbb{R}^{d \times m}$ with the $k$-th column being

\footnotetext{
${ }^{1}$ In this chapter, the dimension of the ambient space can be arbitrary and is not confined to the case $d \in\{2,3\}$.
} 
the relative position vector $z_{k}$. Then $\operatorname{rank}(P)$ and $\operatorname{rank}(Q)$ are constant along any finite time solution $p(t)$ of (3.3).

Proof. Note that $R^{\top}(z) e=\left(H^{\top} \otimes I_{d \times d}\right) Z e$ according to the expression of the rigidity matrix (as shown in Section 2.3.2 of Chapter 2). By defining a diagonal matrix $\bar{E}=$ $\operatorname{diag}\left(e_{1}, e_{2}, \cdots, e_{m}\right) \in \mathbb{R}^{m \times m}$, one can show that

$$
\left(\bar{E} \otimes I_{d \times d}\right)\left(H \otimes I_{d \times d}\right) p=\left(\bar{E} \otimes I_{d \times d}\right) z=Z e
$$

Hence, there holds

$$
\begin{aligned}
R^{\top}(z) e & =\left(H^{\top} \otimes I_{d \times d}\right) Z e \\
& =\left(H^{\top} \otimes I_{d \times d}\right)\left(\bar{E} \otimes I_{d \times d}\right)\left(H \otimes I_{d \times d}\right) p \\
& :=\left(E \otimes I_{d \times d}\right) p
\end{aligned}
$$

where the matrix $E$ is defined as

$$
E=H^{\top} \bar{E} H
$$

Note that $E$ is a symmetric matrix, which has the same structure as the stress matrix in graph rigidity theory [Connelly, 2005]. In later sections we will see the matrix $E$ plays an important role in the stability analysis of equilibrium sets.

By using the above matrix operations, the gradient flow for the formation control system (3.1) can be rewritten as

$$
\dot{p}(t)=-R^{\top}(p) e(p)=-\left(E(p) \otimes I_{d \times d}\right) p
$$

The vector differential equation (3.8) can be equivalently stated as the following differential flow on the matrix space $\mathbb{R}^{d \times n}$ (without the Kronecker product term)

$$
\dot{P}(t)=-P(t) E^{\top}(p(t))=-P(t) E(p(t))
$$

Since the solution of (3.1) is well defined and can be extended to $t \rightarrow \infty$ which excludes the case of finite escape time (see e.g. Krick et al. [2009]), the existence and uniqueness of the solution to (3.9) is well guaranteed. Actually, the matrix $E$ can be written as $E(P)$, i.e. a smooth matrix-valued function $E(P)$ of the variable $P$. Then according to Lemma 8 (see the appendix in Section 3.6), the rank-preserving property of the matrix flow (3.9) follows by observing $B(t)=-E(p(t))$ and $A(t)=0$.

Similarly, the relative position $z$ system (3.4) can be written as

$$
\begin{aligned}
\dot{z}(t) & =-\left(\left(H H^{\top}\right) \otimes I_{d \times d}\right) Z e \\
& =-\left(\left(H H^{\top}\right) \otimes I_{d \times d}\right)\left(\bar{E} \otimes I_{d \times d}\right) z \\
& =-\left(\left(H H^{\top} \bar{E}\right) \otimes I_{d \times d}\right) z
\end{aligned}
$$

The vector differential equation (3.10) can be equivalently stated as the following 
differential flow on the matrix space $\mathbb{R}^{d \times m}$ (without the Kronecker product term)

$$
\dot{Q}(t)=-Q(t)\left(H H^{\top} \bar{E}(t)\right)^{\top}=-Q(t)\left(\bar{E}(t) H H^{\top}\right)
$$

The existence and uniqueness of the solution to (3.11) is also guaranteed by the solution property of (3.4). Then according to Lemma 8 (see the appendix in Section 3.6), the rank-preserving property relating to $\operatorname{rank}(Q(t))$ is proved by observing $B(t)=-\bar{E}(t) H H^{\top}$ and $A(t)=0$.

\subsubsection{Consequences of the rank-preserving property}

If one restricts the discussion for the case of $d=2,3$ (i.e. the 2-D formation control and the 3-D formation control), one can obtain the following invariance properties which are direct consequences of Theorem 3.

Corollary 1. The set of collinear positions is invariant for 2-D formation systems, and the sets of collinear or coplanar positions are invariant for 3-D formation systems. That is, for 2-D formations, if all the agents start with collinear positions, they will always be in collinear positions under the gradient flow (3.3). Similarly, for 3-D formations, if all the agents start with coplanar (resp. collinear) positions, then they will always be in coplanar (resp. collinear) positions under the gradient flow (3.3).

For the formation control system in the presence of distance mismatches (which is termed a mismatched formation flow [Mou et al., 2016]), the rank-preserving property still holds; see the proof in [Helmke et al., 2014].

Remark 1. A similar result on a dimension-invariant property for formation control systems is shown in a recent paper [Chen and Brockett, 2014], which is termed a path-connected property. The authors in [Chen and Brockett, 2014] used Lie group and Lie algebra ideas for proving the property. Here we are using a simpler method based on rank-preserving flow for the proof.

Note that the limiting rank-invariant property of the gradient flow (3.3) for $t \rightarrow \infty$ is not guaranteed. Since any solution $p(t)$ of the real analytic gradient flow converges to an equilibrium point $\bar{p}$, the limit $P(\infty)$ (as well as $Q(p(\infty))$ ) exists with $\operatorname{rank}(P(\infty)) \leq \operatorname{rank}(P(0))$ and $\operatorname{rank}(Q(\infty)) \leq \operatorname{rank}(Q(0)) .{ }^{2}$ In the case of $d=2,3$, this corresponds to the convergence analysis of collinear/coplanar equilibria for the 2-D (and 3-D) formations. These will be discussed in the following sections.

\footnotetext{
${ }^{2}$ One typical example of $\operatorname{rank}(Q(\infty))<\operatorname{rank}(Q(0))$ comes from the formation control problem with unrealizable shapes [Sun et al., 2014c]: If the triangle inequality does not hold for a triangular shape control, then all the agents will converge to a stable collinear equilibrium for which $\operatorname{rank}(Q(\infty))=1$, even if they start with non-collinear positions with $\operatorname{rank}(Q(0))=2$. Note that for such flows the rank-preserving property still holds for any finite time but at the limit $t=\infty$ the rank is lost.
} 


\subsection{Stability analysis for degenerate equilibria}

The results in this section are extended from some previous works [Cao et al., 2011] and [Park et al., 2014]. In [Cao et al., 2011] it shows that for a 2-D triangular formation with achievable shapes, all the agents will globally converge to the correct shape if they start with non-collinear positions. In another paper [Park et al., 2014] we proved that for the 3-D tetrahedral formation, if all the agents start with non-coplanar positions, then they will globally converge to the correct shape. In this section we will continue the analysis to show the convergence property of collinear/coplanar equilibria for general 2-D and 3-D formations when $t \rightarrow \infty$, with the intention to obtain more general results for formation systems with general target shapes that live in an arbitrary dimension.

In this section, we suppose that the formation control system under discussions lives in a $d$-dimensional space in which the target formation shape can be realized in the ambient $d$-dimensional space but not at an ambient space with a lower dimension. We call an equilibrium point $\bar{p}$ with $\operatorname{rank}(\bar{Q}(\bar{p}))<d$ an incorrect degenerate equilibrium, according to the fact that the affine space that embeds $\bar{p}$ has dimension less than $d$ and at such a degenerate equilibrium the potential function is not zero (i.e. the target formation shape is not achieved). First we show a result concerning the existence of such incorrect degenerate equilibria.

Lemma 4. For a formation control system (3.3) with a desired target shape achievable in the $d$-dimensional space but not in an ambient space with a dimension less than $d$, there exist incorrect equilibria $\bar{p}$ for which rank $(\bar{Q}(\bar{p}))<d$. Consequently, there always exist collinear equilibria for 2-D formation systems and collinear/coplanar equilibria for 3-D formation systems.

Proof. Note that in Theorem 3 and Corollary 1, we have shown the rank-invariant properties of the trajectories which start in an affine space with lower dimensions. Also note that the potential function is lower bounded (because the formation centroid is invariant and the potential function is always non-increasing along the solution of the gradient flow). Thus, there exist limit values ${ }^{3}$ of $\bar{p}(\infty)$ which lies in an affine space with lower dimensions, which proves the existence of incorrect degenerate equilibria. For $d=2$, this indicates the existence of collinear equilibria for the formation control system; for $d=3$, this indicates the existence of collinear/coplanar equilibria for the formation control system.

Note that the above Lemma 4 also shows that degenerate equilibria $\bar{p}$ for the $d$ dimensional formation system can be attractive when agents start at degenerate initial positions $p(0)$ with $\operatorname{rank}(Q(p(0)))<d$. The aim of this section is to show that such equilibria are generically unstable when agents start with generic initial positions with $\operatorname{rank}(Q(p(0)))=d$.

Remark 2. One needs to distinguish the set of degenerate equilibria and the set of flip/flex formations. According to the definition of degenerate equilibrium in (3.15), the set of such

\footnotetext{
${ }^{3}$ A gradient system cannot have limit cycles or periodic orbits; see e.g. [Wiggins, 2003, Chapter 15].
} 


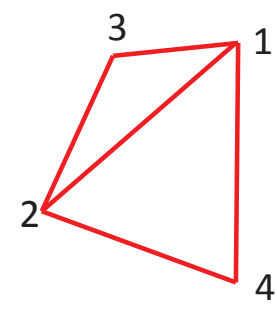

(a)

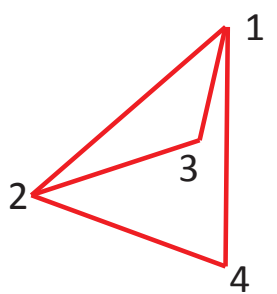

(b)

Figure 3.1: An example of a pair of flip/flex formations. (a) A target formation defined by five inter-agent distances. (b) A flip version of the target formation with the same set of five desired distances. In rigid formation control, they are both considered as correct equilibria for a target formation specified by given desired distances.

equilibrium points is defined by the condition that the agents' positions at such an equilibrium point span an affine space of less than full dimension of its containing space. However, the term flip/flex formations refers to the existence of different realizations (up to translation, rotation and reflection) of a target formation with a given set of distances. Thus, these two concepts are not related. We provide two examples in Figure 3.1 and Figure 3.2 to illustrate these two concepts. Actually, in the distance-based formation setup, a target formation is defined by a set of inter-agent distances, which do not distinguish flip/flex formations. This is because the sets of edges and corresponding distance constraints do not necessarily uniquely define the relative positions of the agents. In other words, the distance constraints do not specify the formation shape up to a rotation, translation, or reflection [Krick et al., 2009; Anderson et al., 2008b]. Since we only focus on local convergence of a gradient-type system, flip/flex ambiguity can be avoided by assuming that the initial formation is closer to the desired shape than the flip/flex version; this guarantees that the formation will converge to the desired shape instead of a flip/flex formation due to the gradient-descent property (see relevant discussions in [Krick et al., 2009]). If one wants to always avoid the flip/flex ambiguity, then the target formation should be globally rigid (for definitions, see e.g. [Anderson et al., 2008b]), or the potential function should be defined in a different way by including constraints on flip/flex formations. Consequently, the corresponding formation controllers should also include additional terms involving the avoidance of flip/flex ambiguity. 


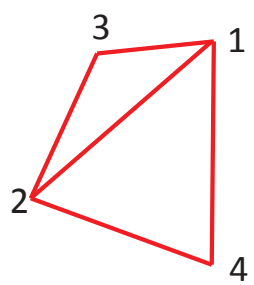

(a)

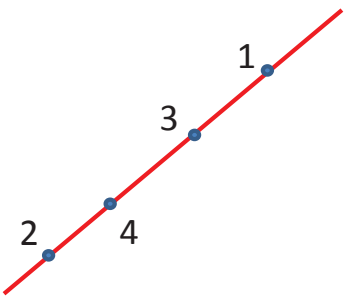

(b)

Figure 3.2: An example of a correct equilibrium and a degenerate equilibrium for a four-agent formation shape with five given distances. (a) The correct formation shape, in which all the five distances are achieved. (b) An example of collinear degenerate equilibrium in $\mathbb{R}^{2}$, in which all the four agents converge to a line formation.

\subsubsection{The equilibrium set}

In this subsection we provide notations to denote different sets of equilibria of (3.3) (i.e. the critical points of $V$ ). ${ }^{4}$ The set for all equilibria is described as

$$
\mathcal{M}=\left\{p \in \mathbb{R}^{d n} \mid R(p)^{\top} e(p)=\left(E(p) \otimes I_{d \times d}\right) p=0\right\}
$$

while the set of correct equilibria is denoted by

$$
\mathcal{M}_{c}=\left\{p \in \mathbb{R}^{d n} \mid e=0\right\}
$$

and the incorrect equilibria set is denoted by

$$
\mathcal{M}_{i}=\left\{p \in \mathbb{R}^{d n} \mid R(p)^{\top} e(p)=\left(E(p) \otimes I_{d \times d}\right) p=0, e \neq 0\right\}
$$

with $\mathcal{M}=\mathcal{M}_{c} \cup \mathcal{M}_{i}$.

According to Lemma 4, a subset of $\mathcal{M}_{i}$ is the set of degenerate equilibria denoted by

$$
\begin{gathered}
\mathcal{M}_{\mathrm{d}}=\left\{p \in \mathbb{R}^{d n} \mid R(p)^{\top} e(p)=\left(E(p) \otimes I_{d \times d}\right) p=0,\right. \\
\operatorname{rank}(P)<d\}
\end{gathered}
$$

Our particular interest will be the case of $d \in\{2,3\}$, i.e. 2-D and 3-D formations. In this case, the set of collinear equilibria (for both 2-D and 3-D formations) is

\footnotetext{
${ }^{4}$ The equilibria form sets, with each set obtainable by translation and rotation of any point in the set (see discussions in e.g. [Summers et al., 2013; Helmke and Anderson, 2013]). For example, for all equilibria for the 2-D formations, there will always be three zero eigenvalues of the Hessian of the potential $V$, corresponding to the rotational and translational invariance. This point will be presented in more detail in Chapter 8.
} 
denoted by

$$
\begin{gathered}
\mathcal{M}_{\text {collinear }}=\left\{p \in \mathbb{R}^{d n} \mid R(p)^{\top} e(p)=\left(E(p) \otimes I_{d \times d}\right) p=0,\right. \\
\operatorname{rank}(Q)=1\}
\end{gathered}
$$

and the set of incorrect coplanar equilibria (for 3-D formations) is denoted by

$$
\begin{gathered}
\mathcal{M}_{\text {coplanar }}=\left\{p \in \mathbb{R}^{d n} \mid R(p)^{\top} e(p)=\left(E(p) \otimes I_{d \times d}\right) p=0,\right. \\
\operatorname{rank}(Q)=2\}
\end{gathered}
$$

Note that for a general 2-D formation with $n \geq 4$ agents, one has $\mathcal{M}_{\text {collinear }} \subset$ $\mathcal{M}_{i}$, and for a general 3-D formation with $n \geq 5$ agents, there holds $\left(\mathcal{M}_{\text {collinear }} \cup\right.$ $\left.\mathcal{M}_{\text {coplanar }}\right) \subset \mathcal{M}_{i} \subset \mathcal{M}$. Since we have assumed that the target formation shape is realized in the given $d$-dimensional ambient space but cannot be realized in an ambient space with a dimension less than $d$, the above analysis means that for a 2-D/3$\mathrm{D}$ general formation there exists an additional incorrect equilibrium set containing the collinear/coplanar equilibria (see [Helmke and Anderson, 2013; Anderson and Helmke, 2014]). It is an open problem to determine analytically the existence and properties of stable incorrect equilibria in rigid formation control. ${ }^{5}$ The following analysis can be seen as a further step towards tackling this problem, and we will show that all the degenerate equilibria are unstable.

\subsubsection{Eigenvalue property of $E$ and the Hessian}

We first show a result on the eigenvalue property of $E$ which holds for formation systems in arbitrary dimensions.

Lemma 5. The matrix $E(\bar{p})$ has at least one negative eigenvalue at an incorrect equilibrium $\bar{p} \in \mathcal{M}_{i}$.

Proof. Suppose to obtain a contradiction that $E(\bar{p})$ is positive semidefinite, which means that $p^{\top}\left(E(\bar{p}) \otimes I_{d \times d}\right) p \geq 0$ for all $p \in \mathbb{R}^{d n}$. According to the structure of the matrix $E$, there holds

$$
\begin{aligned}
p^{\top}\left(E(\bar{p}) \otimes I_{d \times d}\right) p & =p^{\top}\left(H^{\top} \otimes I_{d \times d}\right)\left(\bar{E} \otimes I_{d \times d}\right)\left(H \otimes I_{d \times d}\right) p \\
& =\sum_{(i, j) \in \mathcal{E}} e_{k_{i j}}(\bar{p})\left\|p_{i}-p_{j}\right\|^{2}
\end{aligned}
$$

Note that

$$
\bar{p}^{\top}\left(E(\bar{p}) \otimes I_{d \times d}\right) \bar{p}=0
$$

because $\left(E(\bar{p}) \otimes I_{d \times d}\right) \bar{p}=0$ at an equilibrium $\bar{p}$. Choose $p=p^{*} \in \mathcal{M}_{c}$. Then there

\footnotetext{
${ }^{5}$ Numerical examples on stable incorrect equilibria indeed exist; [Park et al., 2016].
} 
holds

$$
\left\|p_{i}^{*}-p_{j}^{*}\right\|^{2}-\left\|\bar{p}_{i}-\bar{p}_{j}\right\|^{2}=-e_{k_{i j}}(\bar{p})
$$

Thus, one can obtain

$$
\begin{aligned}
p^{* \top}\left(E(\bar{p}) \otimes I_{d \times d}\right) p^{*} & =p^{* \top}\left(E(\bar{p}) \otimes I_{d \times d}\right) p^{*}-\bar{p}^{\top}\left(E(\bar{p}) \otimes I_{d \times d}\right) \bar{p} \\
& =\sum_{(i, j) \in \mathcal{E}} e_{k_{i j}}(\bar{p})\left(\left\|p_{i}^{*}-p_{j}^{*}\right\|^{2}-\left\|\bar{p}_{i}-\bar{p}_{j}\right\|^{2}\right) \\
& =-\sum_{(i, j) \in \mathcal{E}}\left|e_{k_{i j}}(\bar{p})\right|^{2}<0
\end{aligned}
$$

which contradicts the assumption that $E(\bar{p})$ is positive semidefinite. Note that the above strict inequality holds because $\bar{p}$ is an incorrect equilibrium point resulting in at least one nonzero distance error. Hence the statement is proved.

Some further properties of $E$ can be obtained for other particular equilibrium sets. For example, $E\left(p^{*}\right)$ is a zero matrix for $p^{*} \in \mathcal{M}_{c}$. Also at the local maximum (where all the agents are collocated at one point), $E$ is negative semidefinite.

In the sequel, we will use Lemma 5 to study the stability of degenerate equilibria for a general formation system, which includes the stability properties of collinear equilibria for 2-D formations and collinear/coplanar equilibria for 3-D formations. Note that the closed-loop formation control system (3.3) describes a gradient descent flow of the potential function $V$ defined in (3.2), and the equilibrium points of (3.3) are the same as the critical points of $V$. The Jacobian of the right-hand side of the formation system (3.3) is the same as the negative of the Hessian of $V$, which is denoted as $H_{V}(p)$. We show that there is a nice and very useful expression for the Hessian $H_{V}(p)$ (see e.g. [Anderson and Helmke, 2014; Sun et al., 2014c]):

$$
H_{V}(p)=2 R^{\top}(p) R(p)+\left(E(p) \otimes I_{d \times d}\right)
$$

The core idea is the eigenstructure analysis of the Hessian $H_{V}(p)$ of the potential function $V$, which will be detailed in next subsection.

\subsubsection{Degenerate equilibria are unstable}

The nature of an equilibrium (of being a local minimum, a saddle point or a local maximum) for system (3.3) can be determined by the signs of the eigenvalues of the Hessian at that equilibrium. Firstly, we introduce a transformation matrix $T \in$ $\mathbb{R}^{d n \times d n}$, which is used to transform the rigidity matrix into another form:

$$
R T=\left[R_{1} R_{2} \cdots R_{l} \cdots R_{d}\right]=\bar{R}
$$

where $R_{l} \in \mathbb{R}^{m \times n}$ is the sub-matrix of $R$ whose columns consist of the columns of $R$ corresponding to the $l$-th coordinate. Taking 2 -D formation case as an example, $T$ 
is constructed to transform the rigidity matrix: $R T=\left[R_{x}, R_{y}\right]=\bar{R}$, where $R_{x}$ and $R_{y}$ are the sub-matrices whose columns consist of the columns of $R$ corresponding to the coordinates $x$ and $y$, respectively. By doing this, one can obtain a transformed Hessian matrix $\bar{H}_{V}(p)$ as

$$
\begin{aligned}
\bar{H}_{V}(p) & =T^{\top} H_{V}(p) T \\
& =2\left[\begin{array}{c}
R_{1}^{\top} \\
R_{2}^{\top} \\
\vdots \\
R_{d}^{\top}
\end{array}\right]\left[\begin{array}{lll}
R_{1} & R_{2} \cdots & R_{d}
\end{array}\right]+I_{d \times d} \otimes E(p)
\end{aligned}
$$

Since $T$ is a permutation matrix which is orthogonal, the eigenvalues of $H_{V}(p)$ and $\bar{H}_{V}(p)$ are the same; thus in the following we shall consider the eigenvalues of $\bar{H}_{V}(p)$.

In the following analysis, without loss of generality, we will study the stability of the degenerate equilibria $\bar{p}$ with $\operatorname{rank}(\bar{Q})=d-1$; the case for $\operatorname{rank}(\bar{Q})<d-1$ can be handled similarly. Further note that the stability of an equilibrium point is independent of the action of $S E(d)$. In particular, only relative positions matter. We further suppose, again without loss of generality, that the degenerate equilibrium formation lives in the $(d-1)$-dimensional subspace spanned by the basis of the first $d-1$ coordinates. That is, $R_{d}=0$ and one obtains the special expression of the Hessian as follows:

$$
\bar{H}_{V}=\left[\begin{array}{ccccc}
2 R_{1}^{\top} R_{1}+E & 2 R_{1}^{\top} R_{2} & \cdots & 2 R_{1}^{\top} R_{d-1} & \mathbf{0} \\
2 R_{2}^{\top} R_{1} & 2 R_{2}^{\top} R_{2}+E & \cdots & 2 R_{2}^{\top} R_{d-1} & \mathbf{0} \\
\vdots & \vdots & \ddots & \vdots & \vdots \\
2 R_{d-1}^{\top} R_{1} & 2 R_{d-1}^{\top} R_{2} & \cdots & 2 R_{d-1}^{\top} R_{d-1}+E & \mathbf{0} \\
\mathbf{0} & \mathbf{0} & \cdots & \mathbf{0} & E
\end{array}\right]
$$

which will be the key to identify the property of degenerate equilibria.

The following result is a consequence of Lemma 5.

Lemma 6. The matrix $\bar{H}_{V}(\bar{p})$ and consequently the Hessian $H_{V}(\bar{p})$ have at least one negative eigenvalue at an incorrect degenerate equilibrium $\bar{p} \in \mathcal{M}_{d}$. Thus the degenerate equilibria are unstable saddle points.

Proof. It can be seen from the block-diagonal structure of the matrix $\bar{H}_{V}$ in (3.24) that the eigenvalues of $E$ are a part of the eigenvalue set of $\bar{H}_{V}$, and the eigenvalues of $\bar{H}_{V}$ are the same to those of $H_{V}$. Since in Lemma 5 we have proved that $E$ has at least one negative eigenvalue at any incorrect equilibrium in the set $\mathcal{M}_{i}$ which includes the set of (incorrect) degenerate equilibria $\mathcal{M}_{d}, H_{V}(\bar{p})$ has at least one negative eigenvalue at the (incorrect) degenerate equilibrium $\bar{p} \in \mathcal{M}_{d}$. ${ }^{6}$ Thus the incorrect degenerate equilibria are actually saddle points which are unstable.

\footnotetext{
${ }^{6}$ The case for $\operatorname{rank}(\bar{Q})<d-1$ can be handled similarly and the results remain unchanged. This is because the degenerate equilibria with $\operatorname{rank}(\bar{Q})<d-1$ will introduce more negative eigenvalues in the Hessian, and the dimension for the unstable subspaces associated with these negative eigenvalues of the linearized system at such degenerate equilibria will be increasing.
} 
Similar to the definition of degenerate equilibrium, we define agents' degenerate positions in the ambient $d$-dimensional space as positions satisfying $\operatorname{rank}(Q(p))<d$, i.e., the set of agents' positions that span an affine space with dimension less than $d$. All the above lemmas then lead to the following main result.

Theorem 4. Starting with generic initial positions in the d-dimensional space, agents' trajectories will not converge to any degenerate equilibria and their positions will be bounded away from any degenerate positions.

Proof. The linearized version of the formation system (3.8) at an equilibrium point $\bar{p}$ can be described as

$$
\begin{aligned}
\dot{p} & =-H_{V}(\bar{p}) p \\
& =-T \bar{H}_{V}(\bar{p}) T^{\top} p
\end{aligned}
$$

In Lemma 6 it was proved that there exists at least one negative eigenvalue for the Hessian $H_{V}(\bar{p})$ at a degenerate equilibrium $\bar{p}$. We denote the corresponding eigenvector as $v^{-}\left(H_{V}\right)$, where the unstable subspace of the linearized system (3.25) at $\bar{p}$ associated with the eigenvector $v^{-}\left(H_{V}\right)$ can be described as $p=\bar{p}+\operatorname{span}\left(v^{-}\left(H_{V}\right)\right)$. This indicates that the stable subspace, with initial positions chosen in this subspace converging to $\bar{p}$, will have dimension at most $d-1$, let alone the fact that there could be more negative eigenvalues in $H_{V}(\bar{p})$. It follows that such a set of initial positions is a thin set and actually has measure zero in the $d$-dimensional space. In fact, the Center Manifold Theorem [Carr, 1981] implies for the nonlinear system (3.8) that in a small open neighborhood of a degenerate equilibrium $\bar{p}$ there exists an invariant and locally stable (resp. unstable) manifold tangent to the stable (resp. unstable) subspace of (3.25). The set of initial points which lie in the invariant stable manifold and converge to $\bar{p}$ does not contain an interior point of $\mathbb{R}^{d}$ and is a set of first category. By the Baire category theorem [Abraham et al., 1988, Page 34], the union of the stable manifolds of the finitely many incorrect degenerate equilibria is nowhere dense, i.e. it is contained in the complement of a residual set. This shows that the gradient flow (3.8) for generic initial conditions does not converge to an incorrect degenerate equilibrium point.

By combining the results from Theorem 3 and the above arguments, one can conclude that a generic trajectory that starts with a generic initial position $p(0)$ in $\mathbb{R}^{d}$ will always be bounded away from any degenerate positions for which $\operatorname{rank}(Q(t))<$ $d$.

The following results are direct consequences of the above main theorem.

Corollary 2. For 2-D formations, agents' trajectories with generic initial positions in the 2-D space will not approach an incorrect collinear equilibrium.

Corollary 3. For 3-D formations, agents' trajectories with generic initial positions in the 3-D space will not approach an incorrect collinear/coplanar equilibrium.

Figures 3.3 and 3.4 show intuitive explanations of the above two corollaries. 


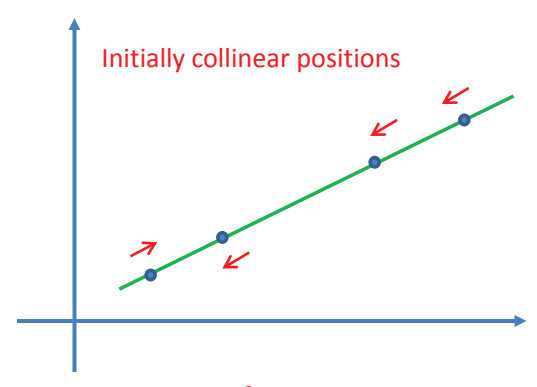

A 2-D formation

(a)

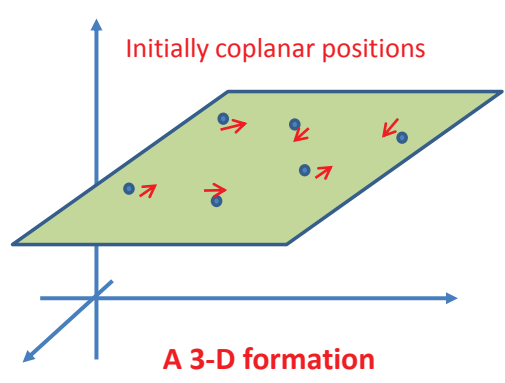

(b)

Figure 3.3: The sets of collinear or coplanar positions are invariant for 2-D/3-D rigid formation systems.

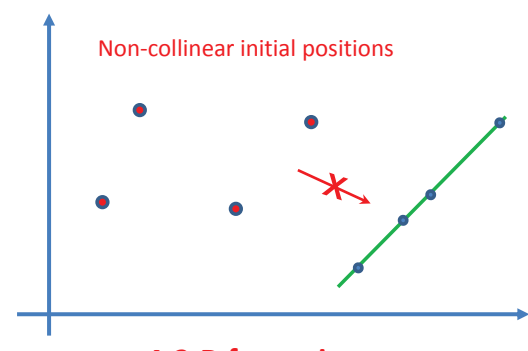

A 2-D formation

(a)

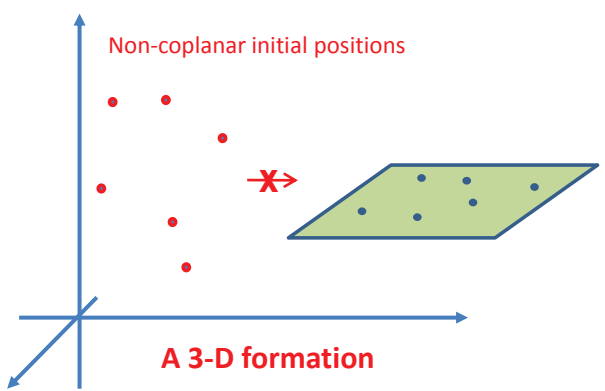

(b)

Figure 3.4: If all agents start at non-collinear (non-coplanar) positions, then their positions will be non-collinear (non-coplanar) at any finite or infinite time. 
Remark 3. One may be concerned with the physical interpretations and applications of the above result for the case of $d>3$, as the real-life implementable formation systems are always in the physical space that has $d=1,2,3$ dimension(s). Here we mention that the results may have implications in other fields. One closely related area is the multidimensional scaling research using an s-stress function [Borg and Groenen, 2005]. Multidimensional scaling problems aim to find a desired configuration with a specific embedding dimension to minimize a stress function, where the stress function usually takes the same form of potential function described in (3.2) as what people in control community often use for formation control. Thus, the results obtained in this chapter can also be employed to determine some equilibrium properties in multidimensional scaling problems.

\subsection{Concluding remarks}

In this chapter we have shown two main results for rigid formation control systems in arbitrary dimensions. The first one is a rank-preserving property, which indicates that if all the agents' positions start in an affine space with a specific dimension, then their trajectories will live in that space with the same dimension. The second main result is on the stability of the degenerate equilibria, which are proven to be unstable. The consequence is that if all the agents start with generic positions in the full dimensional space, then their trajectories will be strictly bounded away from the set of degenerate formations at any finite or infinite time. The results established in this chapter also have applications in other fields, such as equilibrium analysis in multidimensional scaling research.

\subsection{Appendix: background on rank-preserving matrix flow}

In this section we will briefly review some background on rank-preserving flow theory [Helmke and Moore, 1994, Chapter 5].

For integers $1 \leq n \leq \min (M, N)$, let

$$
\mathbb{M}(n, M \times N)=\left\{X \in \mathbb{R}^{M \times N} \mid \operatorname{rank}(X)=n\right\}
$$

denote the set of real $M \times N$ matrices of fixed rank $n$. The following results will be useful in the analysis of some main results in this chapter.

Lemma 7. $\mathbb{M}(n, M \times N)$ is a smooth and connected manifold of dimension $n(M+N-n)$, if $\max (M, N)>1$. The tangent space of $\mathbb{M}(n, M \times N)$ at an element $X$ is

$$
T_{X} \mathbb{M}(n, M \times N)=\left\{\Delta_{1} X+X \Delta_{2} \mid \Delta_{1} \in \mathbb{R}^{M \times M}, \Delta_{2} \in \mathbb{R}^{N \times N}\right\}
$$

A matrix differential equation $\dot{X}=F(t, X)$ evolving on the matrix space $\mathbb{R}^{M \times N}$ is said to be rank-preserving if the rank of every solution $X(t)$ is constant as a function of finite $t$, that is, $\operatorname{rank}(X(t))=\operatorname{rank}(X(0))$ for all $t \geq 0$. The following lemma characterizes such rank-preserving flows (cf. Lemma 1.22 in Chapter 5 of [Helmke and Moore, 1994]). 
Lemma 8. Let $I \subset \mathbb{R}$ be an interval and let $A(t) \in \mathbb{R}^{M \times M}, B(t) \in \mathbb{R}^{N \times N}, t \in I$, be a continuous time-varying family of matrices. Then

$$
\dot{X}(t)=A(t) X(t)+X(t) B(t), \quad X(0) \in \mathbb{R}^{M \times N}
$$

is rank-preserving. Conversely, every rank-preserving differential equation on $\mathbb{R}^{M \times N}$ is of the form (3.27) for matrices $A(t)$ and $B(t)$.

The proof of Lemma 8 is based on the fact that (3.27) defines a time varying vector field on the subset of the tangent space of $\mathbb{M}(n, M \times N)$ described by (3.26). The full proof can be found in [Helmke and Moore, 1994, Page 139].

Remark 4. Note that the above lemma on rank-preserving flows also implies that the limit value $X(\infty)$ (if it exists) has rank less than or equal to $\operatorname{rank}(X(0)$ ). 
34 Invariance principles and equilibrium analysis for formation shape control systems 


\section{Exponential stability for formation control systems with generalized controllers}

\section{Chapter summary}

This chapter discusses generalized controllers for distance-based rigid formation shape stabilization and aims to provide a unified approach for the convergence analysis. We consider two types of formation control systems according to different characterizations of target formations: minimally rigid target formations and nonminimally rigid target formations. For the former case, we firstly prove the exponential stability for rigid formation systems when using a general form of shape controller with certain properties. From this viewpoint, different formation controllers proposed in the previous literature can be included in a unified framework. We then extend the result to the case that the target formation is non-minimally rigid, and show that exponential stability of the formation system is still guaranteed with generalized controllers.

\subsection{Introduction}

\subsubsection{Background and related work}

As reviewed in [Oh et al., 2015], the existing results on formation control can be characterized into position-, displacement-, and distance-based control strategies according to different types of sensed and controlled variables. Among these formation control strategies, distance-based formation control receives particular interest as it does not require all the agents to have the global knowledge of a common coordinate frame and they can measure the relative position to neighboring agents using their own coordinate frames. Thus, in this chapter we focus on distance-based formation control. In particular, we confine our attention to undirected rigid formations, while relevant discussions on directed formation control can be found in e.g. [Mou et al., 2015]. 
With underpinnings from rigid graph theory (see Chapter 2), it has been established that the formation shape can be achieved by controlling a certain set of interagent distances [Olfati-Saber and Murray, 2002; Anderson et al., 2008b; Krick et al., 2009]. In the rigid formation stabilization problem, one typical controller that has been studied extensively in the literature takes the following form (see e.g. [Krick et al., 2009]):

$$
\dot{p}_{i}=\sum_{j \in \mathcal{N}_{i}}\left(p_{j}-p_{i}\right)\left(\left\|p_{j}-p_{i}\right\|^{2}-d_{i j}^{2}\right)
$$

The above controller (4.1), which is derived from a well-defined potential function shown in (2.9), serves as a standard control law for stabilizing rigid formations. The dynamics of the formation control system (4.1) have been investigated in several succeeding papers, e.g. [Dörfler and Francis, 2010; Belabbas et al., 2012; Anderson and Helmke, 2014]. We mention that alternative kinds of formation controllers other than the one in (4.1) are also available, which have been reported sparsely in the literature (see e.g. [Smith et al., 2006; Anderson et al., 2007; Dimarogonas and Johansson, 2010; Tian and Wang, 2013]).

The main objective of this chapter is to analyze general forms of formation controllers to stabilize rigid shapes. The main contributions of this chapter include a unified approach to discuss the convergence and controller performance of generalized formation controllers, and the associated exponential stability of general formation systems when certain properties of the potential function are satisfied. We show that for a large family of formation control systems which generalizes most existing formation controllers proposed in the literature, the exponential stability of the distance error system can be guaranteed. As is well-known in the control field, exponential stability has a robustness property against small perturbations [Khalil, 2002]. Such a robustness property has been employed in recent papers [Mou et al., 2016; Sun et al., 2015b; Garcia de Marina et al., 2015] to study the behavior of rigid undirected formations when there are mismatches or discrepancies between neighbor agents' distance measurements. Exponential stability ensures that the derived distance error system under controller (4.1) with small distance perturbations still converges to a bounded neighbor set of the origin. Note that in the analysis of the formation robustness issue in [Mou et al., 2016; Sun et al., 2015b; Garcia de Marina et al., 2015], the formation control system is limited to the case of the controller in (4.1). To this end, this chapter shows that formation distance error systems with generalized controllers, which include many special forms of formation controllers studied in the literature, also inherit this robustness property as a consequence of the exponential stability.

In this chapter, we first consider formation stabilization control when the target formation is minimally rigid, which is a common assumption in the literature. We list several requirements of the potential function and its gradient function associated with the distributed control for each agent which render an exponential convergence of the formation system. We give an explicit lower bound on the convergence rate, and also discuss several properties of the generalized formation control systems. By deriving a reduced distance error system via the decomposition principle of a rigid 
framework, we then extend the analysis from the minimally rigid case to the nonminimally rigid case, and further prove that the exponential convergence still holds for non-minimally rigid formation control system.

\subsubsection{Chapter organization}

The rest of this chapter is organized as follows. In Section 4.2, we provide detailed analysis on generalized controllers and prove the exponential stability property for minimally rigid formations. Section 4.3 discusses exponential convergence for formation control systems when the target formation is non-minimally rigid. In Section 4.4, two sets of simulation examples are provided to demonstrate the controller performance. Finally, Section 4.5 concludes this chapter.

\subsection{Exponential stability for minimally rigid formations}

In the coordination control of multi-agent systems, the potential-function-based gradient control law is a popular approach to generate distributed controllers for each individual agent for achieving a global task [Sakurama et al., 2015]. We also follow this framework and construct distance potential functions for stabilizing rigid formation shapes. This section aims to provide a unified analysis for the convergence property when generalized controllers are constructed for stabilizing minimally rigid formations. One of the key results in this section is that exponential convergence can be derived for a large family of distributed formation controllers if certain properties are satisfied. To this end, most current results in the literature on formation shape control with different controllers can be included as special cases and studied in a unified framework. In this section, we assume that the target formation is minimally rigid, while this minimal rigidity assumption will be relaxed in the next section.

Let us define a general potential function $V_{k}:\left(-d_{k_{i j}}^{2}, \infty\right) \rightarrow \mathbb{R}_{\geq 0}$ with the following properties:

1. $V_{k}(\varphi)$ is non-negative and continuously differentiable on $\left(-d_{k}^{2}, \infty\right)$;

2. $V_{k}(\varphi)$ and its gradient $g_{k}(\varphi):=\frac{\partial V_{k}(\varphi)}{\partial \varphi}$ are zero if and only if $\varphi=0$;

3. $g_{k}(\varphi)$ is continuously differentiable over its domain and its first derivative is strictly positive around a small neighborhood of the origin ${ }^{1}$.

\footnotetext{
${ }^{1}$ In the journal paper Sun et al. [2016a], we have used the term strictly monotone increasing function to refer to a scalar valued function whose first derivative is strictly positive over its domain. We later realized this terminology is not appropriate and is not consistent with the usual definition of strictly monotone increasing functions. For example, the function $g(x)=x^{3}$ is strictly monotone increasing but its derivative at $x=0$ is zero. We thank Raik Suttner from University of Würzburg for pointing out this terminology issue.
} 
For each agent $i$, the local potential is constructed as

$$
\Theta_{i}=\frac{1}{2} \sum_{j \in \mathcal{N}_{i}} V_{k}\left(e_{k_{i j}}\right)
$$

We assume each agent is modelled by a single integrator, in the form of $\dot{p}_{i}=u_{i}$ where $u_{i}$ is the control input to be designed. According to the gradient control law, each agent moves via the following gradient flow to minimize its local potential function:

$$
\dot{p}_{i}=u_{i}=-\nabla_{p_{i}} \Theta_{i}=-\sum_{j \in \mathcal{N}_{i}}\left(p_{i}-p_{j}\right) g_{k}\left(e_{k_{i j}}\right)
$$

We note that for stabilizing different inter-agent distances, the function $g_{k}$ in the controller can take different forms.

\subsubsection{Review of special controllers in the literature}

The general potential function and the gradient-based controller (4.3) defined above include many formation control strategies proposed in the literature. For example, in [Anderson et al., 2007] the controller is a special case of (4.3) which takes the following form ${ }^{2}$

$$
\dot{p}_{i}=-\sum_{j \in \mathcal{N}_{i}} \frac{p_{i}-p_{j}}{\left\|p_{i}-p_{j}\right\|}\left(\left\|p_{i}-p_{j}\right\|-d_{k}\right)
$$

where the gradient function $g_{k}\left(e_{k}\right)$ is described as

$$
g_{k}\left(e_{k}\right)=1-\frac{d_{k}}{\sqrt{\left(e_{k}+d_{k}^{2}\right)}}
$$

In [Smith et al., 2006], the designed controller takes the following form

$$
\dot{p}_{i}=-\sum_{j \in \mathcal{N}_{i}} \frac{p_{i}-p_{j}}{\left\|p_{i}-p_{j}\right\|^{2}}\left(\left\|p_{i}-p_{j}\right\|^{2}-d_{k}^{2}\right)
$$

where $g_{k}\left(e_{k}\right)$ is

$$
g_{k}\left(e_{k}\right)=1-\frac{d_{k}^{2}}{\left(e_{k}+d_{k}^{2}\right)}
$$

\footnotetext{
${ }^{2}$ We note that the paper [Anderson et al., 2007] considered a control problem of stabilizing a threecoleader formation, which is modelled by a directed cyclic triangle graph. As proved in [Dörfler and Francis, 2009], such a formation graph is a special case of the so-called cooperative graphs, in which all agents act cooperatively and optimize the same potential function in the sense that they behave as if the sensor graph were undirected. One of the main results of [Dörfler and Francis, 2009] states as follows: "(in formation control) every undirected graph, every directed cycle, and every directed open chain is a cooperative graph".
} 
In [Dimarogonas and Johansson, 2008, 2010] and [Tian and Wang, 2013], the following formation system was studied

$$
\dot{p}_{i}=-\sum_{j \in \mathcal{N}_{i}} \frac{p_{i}-p_{j}}{\left\|p_{i}-p_{j}\right\|^{4}}\left(\left\|p_{i}-p_{j}\right\|^{4}-d_{k}^{4}\right)
$$

where $g_{k}\left(e_{k}\right)$ in this case is described by

$$
g_{k}\left(e_{k}\right)=1-\frac{d_{k}^{4}}{\left(e_{k}+d_{k}^{2}\right)^{2}}
$$

Note that the above control functions (4.5), (4.7) and (4.9) have the attractive property of ensuring collision avoidance for the formation system, provided the agents are not collocated initially.

The most commonly-used gradient control law for stabilizing rigid formations involves the potential function in the form of $V_{k}=1 / 2 e_{k}^{2}$, with the derived $g_{k}$ in a simple form:

$$
g_{k}\left(e_{k}\right)=e_{k}
$$

This controller was proposed in [Krick et al., 2009] and was further studied in e.g. [Dörfler and Francis, 2010; Belabbas et al., 2012; Anderson and Helmke, 2014; Cai and de Queiroz, 2014; Cai and De Queiroz, 2015].

Figure 4.1 shows the curves of several typical controller functions mentioned above. As can be observed from Figure 4.1, all the functions $g_{k}\left(e_{k}\right)$ satisfy the desired properties: they are continuously differentiable and have strictly positive first derivative over the domain, and are zero if and only if $e_{k}$ is zero.

In this section we will show that all such controller functions including those mentioned above guarantee an exponential convergence of the formation control system.

\subsubsection{Convergence analysis}

Define a vector $\Phi$ to denote the gradients of the potential functions in each edge:

$$
\Phi(e)=\left[g_{1}\left(e_{1}\right), g_{2}\left(e_{2}\right), \cdots, g_{m}\left(e_{m}\right)\right]^{\top}
$$

By noting that $\frac{\partial V_{k}\left(e_{k}\right)}{\partial p_{i}}=g_{k}\left(e_{k}\right) \frac{\partial e_{k}}{\partial p_{i}}$ and comparing the expression of the single agent system (4.3) with the definition of rigidity matrix (2.3) in Chapter 2, one can derive the following compact form of the overall formation system:

$$
\dot{p}=-R^{\top}(z) \Phi(e)
$$

where $R(z)$ is the rigidity matrix. We first note that because the right-hand side of the formation control system (4.11) with generalized controllers is Lipschitz continuous due to the continuous differentiability of both $\left(p_{i}-p_{j}\right)$ for all $(i, j) \in \mathcal{E}$ and $g_{k}\left(e_{k}(p)\right)$ for all $k$, the solution of (4.11) exists globally.

According to the definition of the error vector $e$ and the derivation of $R$, one can 

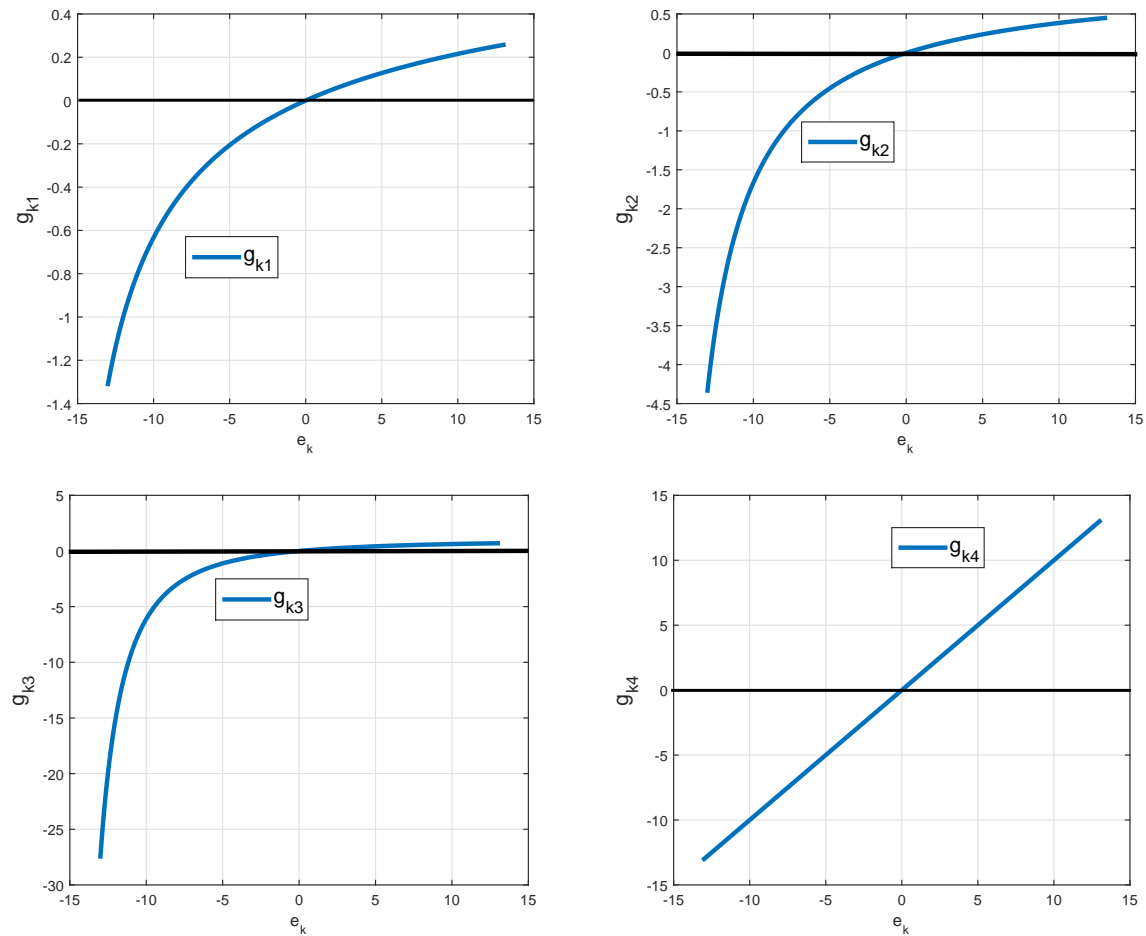

Figure 4.1: Several examples of controller functions $g_{k}$ studied in the literature (which correspond to the controller functions (4.5), (4.7), (4.9) and (4.10) discussed above). In all plots, we let $d_{k}=4$ and thus $e_{k} \in(-16, \infty)$. 
obtain the following compact equation for the distance error vector

$$
\dot{e}=\frac{\partial e}{\partial p} \dot{p}=2 R(z) \dot{p}=-2 R(z) R^{\top}(z) \Phi(e)
$$

Remark 5. It has been proved in [Mou et al., 2016] that when the formation shape is close to the desired one, the entries of the matrix $R(z) R^{\top}(z)$ are continuously differentiable functions of e (see Lemma 2 in Chapter 2). We also denote $Q(e):=R(z) R^{\top}(z)$ to reflect this fact. So the system described in (4.12) is a self-contained system, and we will call it the distance error system in the sequel.

The main result in this section is stated in the following theorem which concerns the exponential stability of the distance error system (4.12).

Theorem 5. Consider the gradient control law (4.3) derived from the general potential functions with the listed properties. Then the distance error system (4.12) has an exponentially stable equilibrium at the origin.

Proof. We divide the whole proof into three main steps.

(I) Proof of asymptotic stability. Define the overall potential function: $V=$ $\frac{1}{2} \sum_{k=1}^{m} V_{k}\left(\left\|z_{k}\right\|^{2}-d_{k}^{2}\right)$ which also serves as a Lyapunov function candidate. Further define a sub-level set $\Psi(\rho)=\{e: V(e) \leq \rho\}$ for some sufficiently small $\rho$, such that for all the points in the set $\Psi(\rho)$ the formation is infinitesimally minimally rigid and close to the target formation. Thus the rigidity matrix is of full row rank, and further $Q(e)=R(z) R^{\top}(z)$ is positive definite (see Lemma 1). By calculating the derivative of $V$ along the trajectories of the distance error system (4.12), one can show

$$
\dot{V}(e)=-\Phi^{\top}(e) Q(e) \Phi(e)
$$

Further let $\lambda$ denote the minimum singular value of the rigidity matrix when $e$ is in the set $\Psi: \lambda=\min _{e \in \Psi} \operatorname{eig}(Q(e))>0$. Note that the set $\Psi(\rho)$ is compact and the existence of such $\lambda$ is guaranteed. Then one has

$$
\dot{V}(e) \leq-\lambda\|\Phi(e)\|^{2}
$$

which indicates that $\dot{V}(e)$ is negative definite for $e \in \Psi \backslash\{0\}$. Thus the asymptotic stability of the equilibrium $e=0$ in the error system (4.12) is proved.

(II) Proof of two inequalities. To show the exponential convergence of the error system (4.12), it suffices to prove the following comparison inequalities (see e.g. Theorem 4.10 of [Khalil, 2002]):

$$
\begin{gathered}
\|\Phi(e)\|^{2} \geq \varrho\|e\|^{2} \\
c_{1}\|e\|^{2} \leq V(e) \leq c_{2}\|e\|^{2}
\end{gathered}
$$

where $\varrho, c_{1}$ and $c_{2}$ are positive constants which need to be determined. The key properties to derive the above inequalities are that the gradient function $g_{k}\left(e_{k}\right)$ is locally 
Lipschitz continuous (due to its continuous differentiability) and its first derivative is strictly positive on $\left(-d_{k}^{2}, \infty\right)$. We follow a similar approach as that in the previous paper [Cao et al., 2011] to show the above inequalities (4.15) and (4.16).

Recall the definition of the Lyapunov function candidate:

$$
V=\frac{1}{2} \sum_{k=1}^{m} V_{k}\left(\left\|z_{k}\right\|^{2}-d_{k}^{2}\right)=\frac{1}{2} \sum_{k=1}^{m} \int_{0}^{e_{k}} g_{k}(s) \mathrm{d} s
$$

Let $\epsilon$ be a positive number, which should satisfy

$$
\epsilon \leq \min \left\{d_{1}^{2}, d_{2}^{2}, \cdots, d_{m}^{2}\right\}
$$

We note that for all $e$ which satisfies $e \in \Psi(\rho) \backslash\{0\}$, the existence for such $\epsilon$ can be guaranteed.

Let

$$
\zeta_{k}=\inf _{|s| \leq \sqrt{\epsilon}} \frac{\mathrm{d} g_{k}(s)}{\mathrm{d} s}, \quad k \in\{1,2, \cdots, m\}
$$

Each $\zeta_{k}$ is positive because each $g_{k}$ has strictly positive first derivative on a neighbor of the origin, which is a subset of the interval $\left(-d_{k}^{2}, \infty\right)$. From this and the fact that $g_{k}(0)=0$ it follows that

$$
\left|g_{k}(s)\right| \geq \zeta_{k}|s|, \quad|s| \leq \sqrt{\epsilon}, \quad k \in\{1,2, \cdots, m\}
$$

Therefore

$$
\int_{0}^{e_{k}} g_{k}(s) \mathrm{d} s \geq \frac{\zeta_{k}}{2} e_{k}^{2}, \quad\left|e_{k}\right| \leq \sqrt{\epsilon}
$$

Choose

$$
2 c_{1}=\min \left\{\frac{\zeta_{1}}{2}, \frac{\zeta_{2}}{2}, \ldots, \frac{\zeta_{m}}{2}\right\}
$$

One has $V(e) \geq c_{1}\|e\|^{2}$. Then the left inequality of (4.16) is true.

Since each $g_{k}$ is Lipschitz continuous and satisfies $g_{k}(0)=0$, there exist positive constants $\delta_{k}$ such that $\left|g_{k}(s)\right| \leq \delta_{k}|s|$ for $|s| \leq \sqrt{\epsilon}$. Then it follows that

$$
\int_{0}^{e_{k}} g_{k}(s) \mathrm{d} s \leq \frac{\delta_{k}}{2} e_{k}^{2}, \quad\left|e_{k}\right| \leq \sqrt{\epsilon}
$$

Choose

$$
2 c_{2}=\max \left\{\frac{\delta_{1}}{2}, \frac{\delta_{2}}{2}, \cdots, \frac{\delta_{m}}{2}\right\}
$$

One has $V(e) \leq c_{2}\|e\|^{2}$. Then the right part of (4.16) is proved.

From (4.19) it is obvious that $\left|g_{k}\left(e_{k}\right)\right|^{2} \geq \zeta_{k}^{2}\left|e_{k}\right|^{2}$. By choosing

$$
\zeta^{2}=\min \left\{\zeta_{1}^{2}, \zeta_{2}^{2}, \cdots, \zeta_{m}^{2}\right\}
$$

one has $\|\Phi(e)\|^{2} \geq \zeta^{2}\|e\|^{2}$. Let $\varrho=\zeta^{2}$, which proves the inequality in (4.15).

(III) Proof of exponential stability. This step is standard from the results of the 
above two steps. By combining the inequalities in (4.14), (4.15) and (4.16), one obtains that

$$
\dot{V}(e) \leq-\lambda \varrho\|e\|^{2} \leq-\frac{\lambda \varrho}{c_{2}} V(e)
$$

Thus, it follows that

$$
\begin{aligned}
\|e(t)\| \leq\left(\frac{V(e(t))}{c_{1}}\right)^{\frac{1}{2}} & \leq\left(\frac{V(e(0))}{c_{1}} \exp \left(-\frac{\lambda \varrho}{c_{2}} t\right)\right)^{\frac{1}{2}} \\
& \leq\left(\frac{c_{2}\|e(0)\|^{2}}{c_{1}} \exp \left(-\frac{\lambda \varrho}{c_{2}} t\right)\right)^{\frac{1}{2}}
\end{aligned}
$$

which indicates the following

$$
\|e(t)\| \leq\left(\frac{c_{2}}{c_{1}}\right)^{\frac{1}{2}} \exp (-\gamma t)\|e(0)\|
$$

with the exponential convergence rate no less than $\gamma=\frac{\lambda \varrho}{2 c_{2}}$.

Remark 6. A recent paper [Oh and Ahn, 2014a] also discussed general controllers to stabilize rigid formation shapes. In [Oh and Ahn, 2014a], the potential function is assumed to be positive definite and analytic (see Assumption 3.1 in [Oh and Ahn, 2014a]), from which the authors proved the local asymptotic convergence of the formation system by exploring the Łojasiewicz inequality [Chill, 2003] for gradient systems. We also note that finite-time convergence can be obtained for formation control systems with another set of potential functions and gradient controllers. Relevant discussions on finite-time convergence can be found in [Sun et al., 2016b].

Note that the convergence of inter-agent distance errors of itself does not directly guarantee the convergence of agents' positions $p(t)$ to some fixed points, even though it does guarantee convergence to a correct formation shape. A sufficient condition for this strong convergence of the position system (4.11) to a stationary formation is guaranteed by the boundedness of the control function and the exponential convergence as proved above, which indicates that the right-hand side of (4.11) is exponentially decaying. This ensures that the solution of (4.11) exists and is finite when $t \rightarrow \infty$. To sum up, one has the following lemma on the convergence of (4.11) as a consequence of Theorem 5.

Lemma 9. Suppose the initial formation shape is close to the target formation shape. The generalized control law (4.3) guarantees the convergence of $p(t)$ to a fixed point.

The above Theorem 5 and Lemma 9 show local convergence to the target formation shape, by assuming that the initial formation shape is close to the target formation shape. In general, the formation control system (4.11) exhibits multiple equilibria. The set for all equilibria is described as $\mathcal{M}=\left\{p \in \mathbb{R}^{d n} \mid R(p)^{\top} g(e(p))=0\right\}$. We call the set of equilibria at which the correct shape is attained the correct equilibrium 
set ${ }^{3}$, and we denote it by $\mathcal{M}_{c}=\left\{p \in \mathbb{R}^{d n} \mid g(e(p))=0\right\}$. Correspondingly, the set of equilibria at which the correct shape is not attained is referred to as incorrect equilibrium set, and it is denoted by $\mathcal{M}_{i}=\left\{p \in \mathbb{R}^{d n} \mid R(p)^{\top} g(e(p))=0, g(e(p)) \neq 0\right\}$.

In the case that the target formation shape is minimally and infinitesimally rigid, one can show the following result which partly characterizes a converged formation at an equilibrium.

Lemma 10. Suppose the target formation is minimally and infinitesimally rigid. Then the formation system (4.3) with generalized controllers converges to either a correct equilibrium in $\mathcal{M}_{c}$, or an incorrect equilibrium in $\mathcal{M}_{i}$ at which the converged formation shape is not infinitesimally rigid.

Proof. Note that $\mathcal{M}=\mathcal{M}_{c} \cup \mathcal{M}_{i}$. Theorem 5 actually proves that an equilibrium point in the set of correct equilibrium $\mathcal{M}_{c}$ is locally exponentially convergent. Note that according to the property of the gradient control function $g(e)$, the condition $g(e(p))=0$ (resp. $g(e(p)) \neq 0$ ) to characterize a correct equilibrium (resp. an incorrect equilibrium) is equivalent to the condition $e(p)=0$ (resp. $e(p) \neq 0)$. At an incorrect equilibrium in $\mathcal{M}_{i}$, the transpose of the rigidity matrix, $R^{\top}$, has a non-trivial null vector $g(e(p)) \neq 0$. Thus, at such an equilibrium $R$ is not of full row rank, which violates the rank condition shown in Theorem 1 on infinitesimal rigidity in Chapter 2. Hence, at an incorrect equilibrium characterized by $e(p) \neq 0$ (or equivalently, $g(e(p)) \neq 0)$, the formation shape is not infinitesimally rigid.

Remark 7. We mention here three significant aspects of the above result stated in Theorem 5. First, it generalizes many existing controllers reviewed in Subsection 4.2.1 in a unified framework and for the first time it proves the exponential convergence for a large family of formation control systems via a unified approach. Second, the exponential stability obtained from the generalized controller has important robustness consequences in case of distance perturbations, which extends the robustness behavior analysis in formation systems observed in [Mou et al., 2016; Sun et al., 2015b], where the controllers were confined to be the one of (4.10). In the next chapter, we will then discuss in detail the robustness issue for 3-D rigid formation systems. Third, we note that for stabilizing different inter-agent distances, the function $g_{k}$ in the controller can take different forms. This implies one can use a group of heterogeneous agents for implementing a formation control strategy in the sense that each agent may have different sensing/controlling performances for controlling different interagent distances as reflected by different $g_{k}$.

\subsubsection{Properties of the formation control system with generalized con- trollers}

We show some intrinsic properties of the formation system with the generalized controllers.

\footnotetext{
${ }^{3}$ Note that the realization of a target formation may not be unique due to e.g. flip ambiguity [Hendrickson, 1992]. Thus the correct formation shape may not be unique.
} 
Lemma 11. The formation stabilization system designed in (4.3) (and in the compact form (4.11)) with the generalized controller has the following properties:

1. The controller is distributed and each agent requires only relative position measurements with respect to its neighboring agents.

2. The center of the mass of the formation is stationary.

3. The measurement and control for each agent are independent of any global coordinates. That is, each agent can use its own coordinate system to measure the necessary relative positions and to implement the control.

The proof is omitted here, as it follows similar ideas to [Cao et al., 2011] and in particular to the proof of [Sun et al., 2014b, Lemma 4]. Note that Part 3 of Lemma 11 implies the proposed formation system (4.3) with generalized controllers guarantees the $S E(N)$ invariance (i.e. translational and rotational invariance) of the controller, which is a nice property to enable convenient implementation for networked control systems without coordinate alignment for each individual agent [Vasile et al., 2015].

Remark 8. The result in this section also provides insights to design other types of formation controllers with guaranteed exponential stability which are not covered in the literature. For example, we can show that by using the following controller

$$
\begin{aligned}
\dot{p}_{i}(t) & =u_{i}(t) \\
& =\sum_{j \in \mathcal{N}_{i}} \frac{p_{j}(t)-p_{i}(t)}{\left\|p_{j}(t)-p_{i}(t)\right\|}\left(\left\|p_{j}(t)-p_{i}(t)\right\|^{2}-d_{k}^{2}\right)
\end{aligned}
$$

the distance error system is (locally) exponentially stable. The formation control system (4.28) has the property that the bearing information and range information are decoupled in the controller. In this case, the function $g_{k}$ is constructed as

$$
g_{k}\left(e_{k}\right)=\frac{e_{k}}{\left(e_{k}+d_{k}^{2}\right)^{\frac{1}{2}}}=\left(e_{k}+d_{k}^{2}\right)^{\frac{1}{2}}-\frac{d_{k}^{2}}{\left(e_{k}+d_{k}^{2}\right)^{\frac{1}{2}}}
$$

which is continuously differentiable and has strictly positive first derivative on $\left(-d_{k}^{2}, \infty\right)$. Thus all the conditions stated in the beginning of Section 4.2 are satisfied, and the exponential convergence then follows.

As another example, one can consider the following distributed formation controller

$$
\dot{p}_{i}(t)=u_{i}(t)=\sum_{j \in \mathcal{N}_{i}}\left(p_{j}(t)-p_{i}(t)\right)\left(\left\|p_{j}(t)-p_{i}(t)\right\|-d_{k}\right)
$$

which also guarantees that the distance error system is (locally) exponentially stable. In this case, the function $g_{k}$ is constructed as

$$
g_{k}\left(e_{k}\right)=\frac{e_{k}}{\left\|p_{j}(t)-p_{i}(t)\right\|+d_{k}}=\frac{e_{k}}{\sqrt{\left(e_{k}+d_{k}^{2}\right)}+d_{k}}
$$



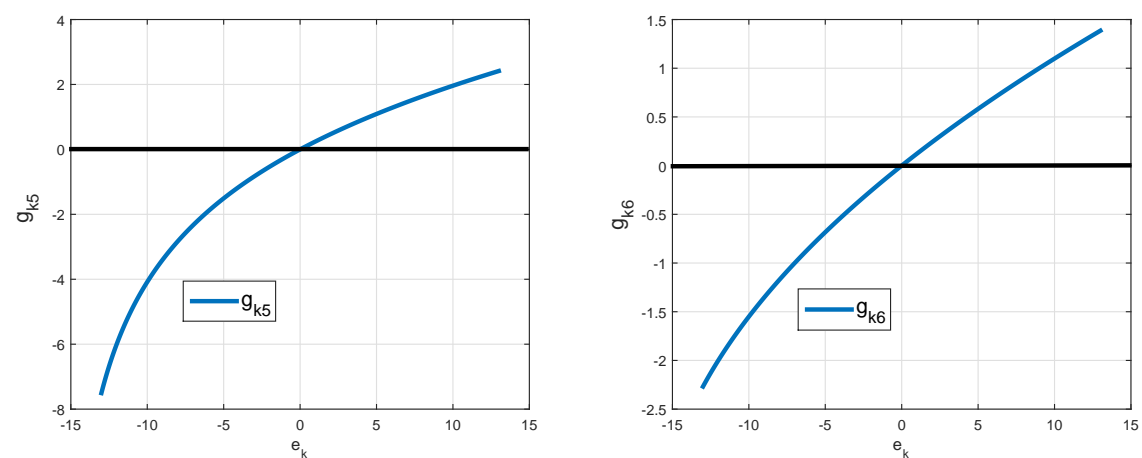

Figure 4.2: Two novel controller functions $g_{k}$ with guaranteed exponential convergence of the distance error system. In both plots, we let $d_{k}=4$ and thus $e_{k} \in(-16, \infty)$. The left figure shows the proposed function in (4.29), and the right figure shows the proposed function in (4.31).

which satisfies all the conditions stated above. The curves of these two novel control functions are depicted in Figure 4.2.

Remark 9. We note that there are also other forms of formation controllers that cannot be described by (4.3). For example, in [Bishop et al., 2015], the controller takes the form of

$$
\dot{p}_{i}=-\Omega\left(\theta_{i}\right) \sum_{j \in \mathcal{N}_{i}}\left(p_{i}-p_{j}\right) e_{k_{i j}}
$$

where $\Omega\left(\theta_{i}\right)$ is a matrix (constructed from some rotation matrices) which should be positive definite, and this relaxed controller also leads to exponential convergence for the formation system.

\subsection{Exponential stability for non-minimally rigid formations}

In this section we aim to extend the results from minimally rigid case to non-minimally rigid case, and prove that the exponential convergence of the distance error system still holds. The analysis in this section is partly motivated by [Mou et al., 2016] and [Park and Ahn, 2014].

Before starting the analysis, we recall the following result which is rephrased from Theorem 2.3 of [Hendrickson, 1992].

Lemma 12. ([Hendrickson, 1992]) Every rigid framework $(\mathcal{G}, p)$ has a rigid subframework with exactly $d n-d(d+1) / 2$ edges.

An example for the graph decomposition of a 4-agent non-minimally rigid framework is shown in Figure 4.3.

Suppose the framework $(\mathcal{G}, p)$ is infinitesimally rigid but not minimally rigid. According to Lemma 12, there exists a subframework which is minimally rigid with 


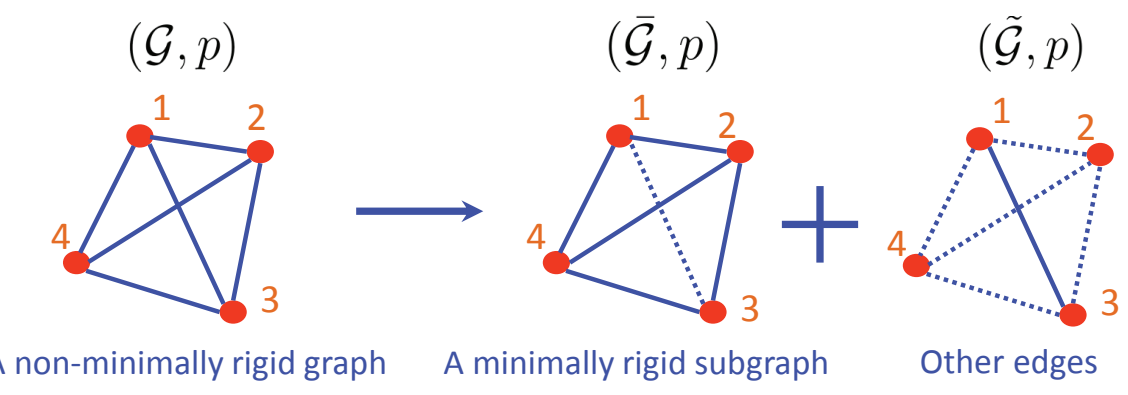

Figure 4.3: An example of graph decomposition of a non-minimally rigid framework.

The dashed lines in the decomposed subgraphs indicate the removed edges.

exactly $d n-d(d+1) / 2$ edges. We choose one such minimally rigid subframeworks and denote it by $(\overline{\mathcal{G}}, p)$, where $\overline{\mathcal{G}}=(\mathcal{V}, \overline{\mathcal{E}})$ with $|\overline{\mathcal{E}}|=d n-d(d+1) / 2$ is a subgraph of $\mathcal{G}$. The remaining part of the framework with $m-(d n-d(d+1) / 2)$ edges is denoted as $(\tilde{\mathcal{G}}, p)$ where $\tilde{\mathcal{G}}=(\mathcal{V}, \tilde{\mathcal{E}})$ with $|\tilde{\mathcal{E}}|=m-(d n-d(d+1) / 2)$. Note that $\mathcal{E}=\overline{\mathcal{E}} \cup \tilde{\mathcal{E}}$.

We denote $\bar{e} \in \mathbb{R}^{|\overline{\mathcal{E}}|}$ as the sub-vector of $e$ whose $|\overline{\mathcal{E}}|$ entries are the entries in $e$ corresponding to the edges in $\overline{\mathcal{G}}$. Similarly, we write $\tilde{e} \in \mathbb{R}^{|\tilde{\mathcal{E}}|}$ as the sub-vector of $e$ whose $|\tilde{\mathcal{E}}|$ entries are the entries in $e$ corresponding to the edges in $\tilde{\mathcal{G}}$. There exist two selection matrices, $\bar{P} \in \mathbb{R}^{|\overline{\mathcal{E}}| \times m}$ and $\tilde{P} \in \mathbb{R}^{|\tilde{\mathcal{E}}| \times m}$, such that the partition can be described as

$$
\bar{e}=\bar{P} e, \quad \tilde{e}=\tilde{P} e
$$

Note that according to the structure of $\bar{P}$ and $\tilde{P}$, the matrix $P:=\left[\bar{P}^{\top} \tilde{P}^{\top}\right]$ is a permutation matrix. Therefore, there hold $\bar{P} \bar{P}^{\top}=I_{|\overline{\mathcal{E}}| \times|\overline{\mathcal{E}}|}, \tilde{P} \tilde{P}^{\top}=I_{|\tilde{\mathcal{E}}| \times|\tilde{\mathcal{E}}|}, \bar{P} \tilde{P}^{\top}=0_{|\overline{\mathcal{E}}| \times|\tilde{\mathcal{E}}|}$ and $e=\bar{P}^{\top} \bar{e}+\tilde{P}^{\top} \tilde{e}$ because $\bar{P}^{\top} \bar{P}+\tilde{P}^{\top} \tilde{P}=I_{m \times m}$. Following a similar procedure, we can also partition the gradient vector $\Phi$ into two sub-vectors $\bar{\Phi}(\bar{e}):=\bar{P} \Phi$ and $\tilde{\Phi}(\tilde{e}):=\tilde{P} \Phi$, corresponding to the subframeworks $(\overline{\mathcal{G}}, p)$ and $(\tilde{\mathcal{G}}, p)$, respectively.

We also denote the rigidity matrix for the subframework $(\overline{\mathcal{G}}, p)$ as $\bar{R} \in \mathbb{R}^{\overline{\mathcal{E}} \times d n}$, and the rigidity matrix for $(\tilde{\mathcal{G}}, p)$ as $\tilde{R} \in \mathbb{R}^{\tilde{\mathcal{E}} \times d n}$. By following similar arguments as above, there holds $\bar{R}=\bar{P} R$ and $\tilde{R}=\tilde{P} R$. Then one can derive the dynamical equation for $\bar{e}$ :

$$
\begin{aligned}
\dot{\bar{e}}=\bar{P} \dot{e} & =-2 \bar{P} R R^{\top}\left(\bar{P}^{\top} \bar{\Phi}(\bar{e})+\tilde{P}^{\top} \tilde{\Phi}(\tilde{e})\right) \\
& =-2 \bar{R} \bar{R}^{\top} \bar{\Phi}(\bar{e})-2 \bar{R} \tilde{R}^{\top} \tilde{\Phi}(\tilde{e})
\end{aligned}
$$

According to Lemma 2 in Chapter 2, there exists a smooth function $\psi$ such that $\tilde{e}=\psi(\bar{e})$ holds locally around $\bar{e}=0$. Note that $\psi(\bar{e})=0$ if and only if $\bar{e}=0$. Also $\tilde{R}=\frac{\partial \tilde{e}}{\partial p}=\frac{\partial \psi}{\partial \bar{e}} \frac{\partial \bar{e}}{\partial p}=F \bar{R}$ where $F:=\frac{\partial \psi}{\partial \bar{e}}$. Furthermore, Lemma 2 implies that all entries of $\bar{R} \bar{R}^{\top}$ and $\bar{R} \tilde{R}^{\top}=\bar{R} \bar{R}^{\top} F^{\top}$ are functions of $\bar{e}$. Thus, the reduced-order distance error system (4.33) is self-contained, and can be rewritten as

$$
\dot{\bar{e}}=-2 \bar{Q}(\bar{e}) \bar{\Phi}(\bar{e})-2 \bar{Q}(\bar{e}) F^{\top} \tilde{\Phi}(\psi(\bar{e}))
$$


where $\bar{Q}(\bar{e})=\bar{R} \bar{R}^{\top}$.

The main result in this section concerns the following exponential stability statement.

Theorem 6. Consider the gradient control (4.3) derived from the general potential functions with the listed properties. The distance error system (4.34) for the non-minimally rigid formation has an exponential stable equilibrium at the origin.

Proof. The proof for the local exponential stability of the equilibrium point $\bar{e}=0$ is through the linearization approach, which follows similarly to the proof of [Mou et al., 2016, Theorem 2].

The calculation of the Jacobian matrix of the reduced-order error system (4.34) at the equilibrium $\bar{e}=0$ reveals

$$
\begin{aligned}
J(\bar{e}):= & \left.\frac{\partial\left(-2 \bar{Q}(\bar{e}) \bar{\Phi}(\bar{e})-2 \bar{Q}(\bar{e}) F^{\top} \tilde{\Phi}(\psi(\bar{e}))\right)}{\partial \bar{e}}\right|_{\bar{e}=0} \\
= & -\left.2\left(\frac{\partial \bar{Q}(\bar{e})}{\partial \bar{e}} \bar{\Phi}(\bar{e})+\frac{\partial\left(\bar{Q}(\bar{e}) F^{\top}\right)}{\partial \bar{e}} \tilde{\Phi}(\psi(\bar{e}))\right)\right|_{\bar{e}=0} \\
& -\left.2\left(\bar{Q}(\bar{e}) \frac{\partial \bar{\Phi}(\bar{e})}{\partial \bar{e}}+\bar{Q}(\bar{e}) F^{\top} \frac{\partial \tilde{\Phi}(\psi(\bar{e}))}{\partial \bar{e}}\right)\right|_{\bar{e}=0}
\end{aligned}
$$

Let $\Psi=\left\{\bar{e} \in \mathbb{R}^{|\overline{\mathcal{E}}|} \mid\|\bar{e}\|^{2}<r\right\}$ with a small positive $r>0$ denote a small neighborhood set around the desired equilibrium $\bar{e}=0$. We first note in $\Psi$ the solution to the system (4.34) is bounded. Furthermore, the Jacobian matrix $J(\bar{e})$ is bounded and Lipschitz continuous on the same set $\Psi$ (due to the smoothness of the function $\psi$, and continuous differentiability of the gradient function $g_{k}$ ). These system properties allow us to employ the Linearization Theorem [Khalil, 2002, Theorem 4.13] to prove the local exponential convergence of (4.34).

By noting that $\left.\bar{\Phi}(\bar{e})\right|_{\bar{e}=0}=0$ and $\left.\tilde{\Phi}(\psi(\bar{e}))\right|_{\bar{e}=0}=0$, the expression of the above Jacobian matrix can be simplified to

$$
J(\bar{e}):=-\left.2 \bar{Q}(\bar{e})\left(\frac{\partial \bar{\Phi}(\bar{e})}{\partial \bar{e}}+F^{\top} \frac{\partial \tilde{\Phi}(\psi(\bar{e}))}{\partial \psi} F\right)\right|_{\bar{e}=0}
$$

Note that $\left.\frac{\partial \bar{\Phi}(\bar{e})}{\partial \bar{e}}\right|_{\bar{e}=0}$ is a diagonal matrix, with the $k$ th diagonal entry being $\frac{\mathrm{d} g_{k}(s)}{\mathrm{d} s}$. Because of the third property of the potential listed in the beginning of Section 4.2, the derivative $\frac{\mathrm{d} g_{k}(s)}{\mathrm{d} s}$ evaluated at $s=0$ is positive. Thus $\left.\frac{\partial \bar{\Phi}(\bar{e})}{\partial \bar{e}}\right|_{\bar{e}=0}$ is a positive definite diagonal matrix. The same reasoning implies that $\left.\frac{\partial \tilde{\Phi}(\psi(\bar{e}))}{\partial \psi}\right|_{\bar{e}=0}$ is also a positive definite diagonal matrix. Thus $\frac{\partial \bar{\Phi}(\bar{e})}{\partial \bar{e}}+F^{\top} \frac{\partial \tilde{\Phi}(\psi(\bar{e}))}{\partial \psi} F$ is positive definite evaluated at $\bar{e}=0$. Note that $\bar{Q}(0)$ is positive definite due to the infinitesimal and minimal rigidity of the subframework $(\overline{\mathcal{G}}, p)$ of the target formation. According to [Horn and Johnson, 2012, Corollary 7.6.2, Page 486], all eigenvalues of the product ma- 
$\left.\operatorname{trix} \bar{Q}(\bar{e})\left(\frac{\partial \bar{\Phi}(\bar{e})}{\partial \bar{e}}+F^{\top} \frac{\partial \tilde{\Phi}(\psi(\bar{e}))}{\partial \psi} F\right)\right|_{\bar{e}=0}$ are real and positive. Thus, the Jacobian matrix $\left.J(\bar{e})\right|_{\bar{e}=0}$ is a stability matrix with all of its eigenvalues being negative real, which implies that $\bar{e}=0$ is an exponentially stable equilibrium for the linearized system $\dot{\bar{e}}=J(\bar{e}) \bar{e}$ of (4.34). According to [Khalil, 2002, Theorem 4.13], the equilibrium point $\bar{e}=0$ is locally exponentially stable for the nonlinear system (4.34). The proof is thus complete.

\subsection{Simulation examples}

In this section we provide two simulations to show the behavior of certain formation systems with generalized controllers. Firstly consider a 4-agent formation shape in the 2-D space modelled by a complete graph. The desired distances are given by $d_{12}=d_{34}=3, d_{23}=d_{14}=4, d_{13}=d_{24}=5$, which correspond to a rectangular target shape. Note that in this case the underlying graph for the target formation has 6 edges, while the maximum rank of the associated rigidity matrix is 5 . Thus the formation graph is not minimally rigid, but it contains a minimally rigid subgraph. By the analysis in Section 4.3, the local exponential convergence of the formation system with generalized controllers can be shown.

In the simulation, the initial conditions for each agent are chosen as $p_{1}(0)=$ $[0,0]^{\top}, p_{2}(0)=[-1,4]^{\top}, p_{3}(0)=[5,3]^{\top}$ and $p_{4}(0)=[3,0]^{\top}$, so that the initial formation is close to the target one. We assume that for different edges the agents are using different controller forms. Specifically, for the edges $(1,3)$ and $(2,4)$ the controller contributions in the related agents are based on $(4.5)$, for the edges $(1,2)$ and $(3,4)$ the controller contributions are based on $(4.7)$, and for edges $(2,3)$ and $(1,4)$ the agents use the control function of (4.10). The trajectories of each agent and the final shape are depicted in Figure 4.4. The trajectories of each distance error are depicted in Figure 4.5, which shows an exponential convergence to the origin.

We then consider a formation control example in the 3-D space. The target formation shape is supposed to be a double tetrahedron formation with 9 edges, with the desired distances for each edge being 2. Note that in this case the underlying graph for the target formation shape is minimally rigid, since the maximum rank of the associated rigidity matrix, which equals the number of edges, can be attained by a realization of the target formation. For the controller design, we assume that all the agents adopt the novel control function of (4.30) proposed in Remark 8. In the simulation, the initial conditions for each agent are chosen as $p_{1}(0)=[0,-1.0,0.5]^{\top}, p_{2}(0)=[1.8,1.6,-0.1]^{\top}, p_{3}(0)=[-0.2,1.8,0.05]^{\top}$, $p_{4}(0)=[1.2,1.9,1.7]^{\top}$ and $p_{5}(0)=[-1.0,-1.5,-1.2]^{\top}$, so that the initial formation is close to the target one. Figure 4.6 illustrates the trajectories of each agent, together with the initial shape and final shape. The trajectories of each distance error are depicted in Figure 4.7, which also shows an exponential convergence to the origin. 


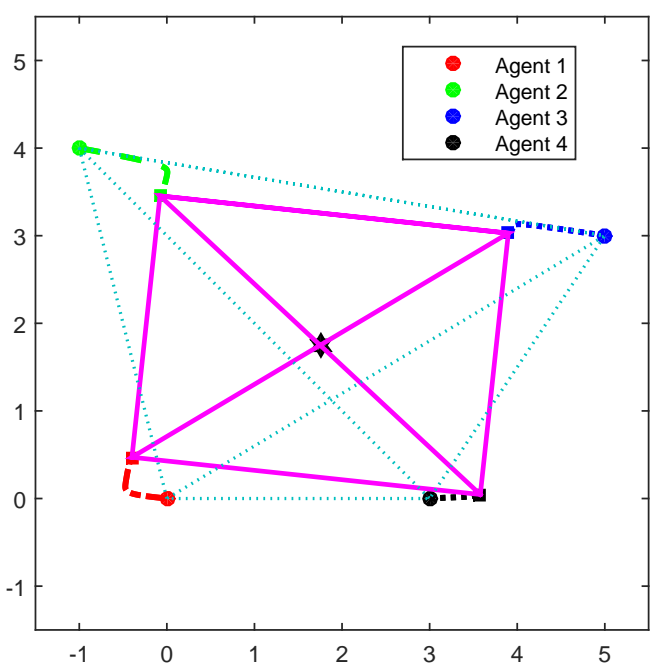

Figure 4.4: Simulation on stabilization control of an 4-agent rectangular formation shape. The initial and final positions are denoted by circles and squares, respectively. The initial formation is denoted by dotted blue lines, and the final formation is denoted by red solid lines. The black star denotes the formation centroid, which is stationary.

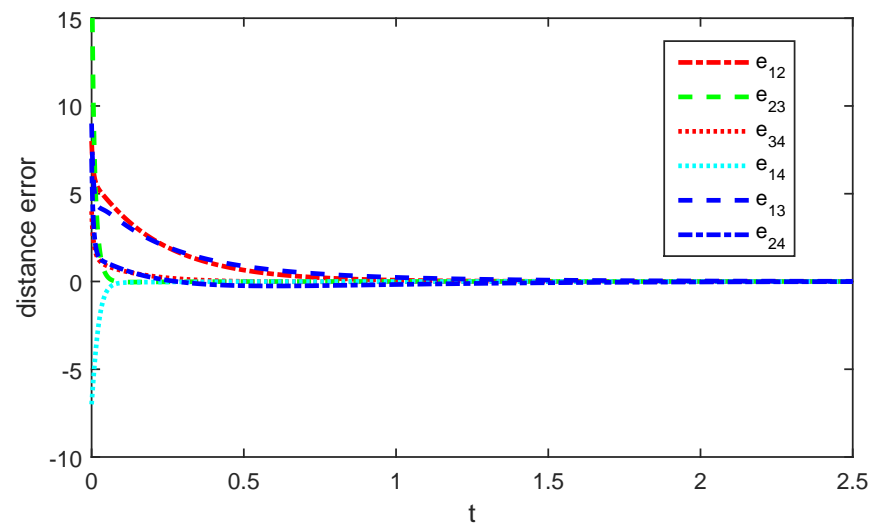

Figure 4.5: Exponential convergence of the distance errors for 2-D rectangular formation control. 


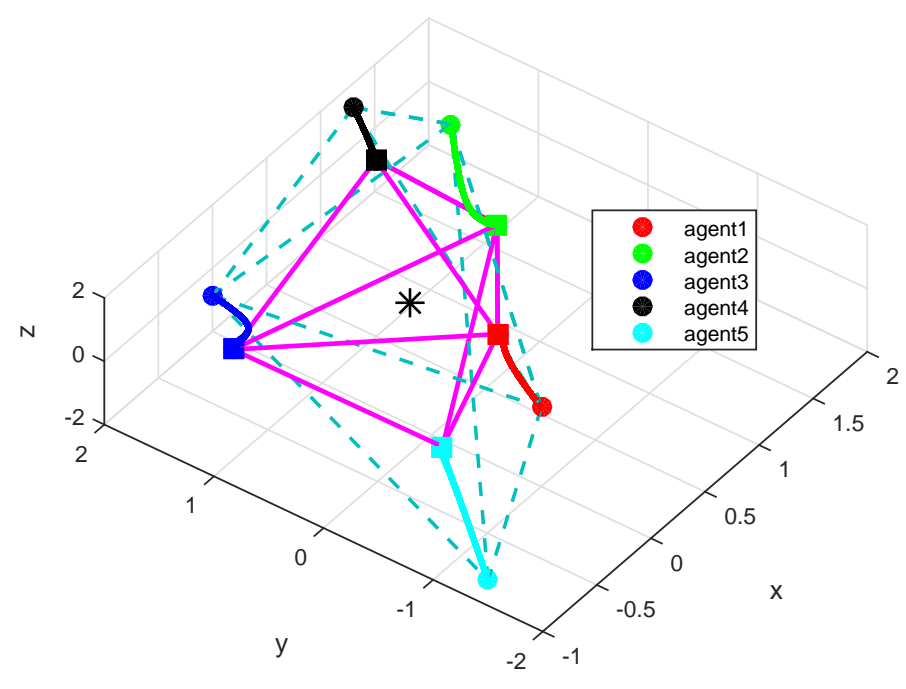

Figure 4.6: Simulation on stabilization control of a double tetrahedron formation in 3-D space. The initial and final positions are denoted by circles and squares, respectively. The initial formation is denoted by dashed lines, and the final formation is denoted by red solid lines. The black star denotes the formation centroid, which is stationary.

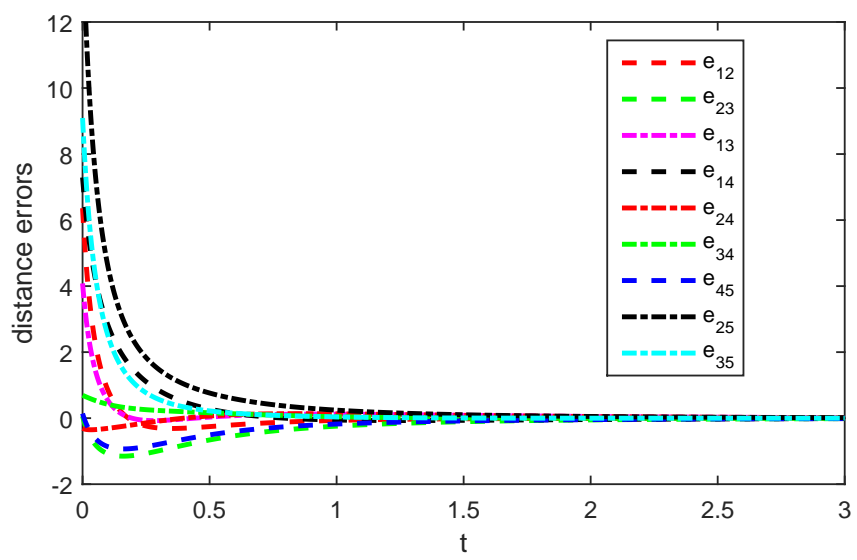

Figure 4.7: Exponential convergence of the distance errors for 3-D tetrahedron formation control. 


\subsection{Concluding remarks}

In this chapter we show a unified approach to analyze the convergence of distancebased rigid formation control systems with distributed formation controllers. The general forms of the formation controllers encompass most controllers with special forms proposed in the literature for stabilizing formation shapes. Furthermore, in the framework studied in this chapter, the stabilization control of each distance can be assigned with different distance potentials which also enables an implementation with heterogenous agents in formation control. Two cases of target formations are discussed in detail: minimally rigid formations and non-minimally rigid formations. For both cases, we prove the exponential convergence property of the formation system with generalized controllers. One significant consequence of the exponential stability is that the robustness properties of formation control analyzed in [Mou et al., 2016; Sun et al., 2015b] can be extended to a large family of rigid formation systems with generalized controllers obtained in this chapter. The robustness issue for 3-D rigid formation control systems in the presence of mismatched distances will be discussed in the next chapter. 


\section{Part II}

\section{Formation Control Systems: Some Practical Considerations}





\section{Robustness issues for 3-D undirected formations with distance mismatches}

\section{Chapter summary}

This chapter discusses a key robustness issue of 3-D undirected rigid formations: what happens in the case that neighboring agent pairs have slight biases in their distance measurements or have slightly differing views of the desired inter-agent distances they are tasked to maintain? The main aim in this chapter is to examine the collective motion behavior induced by mismatched distances. We show that the formation shape will converge exponentially fast to a rigid one, while additional rigid helical motions of the final formation will occur. We further discuss the convergence to the equilibrium motions, and derive certain motion parameter formulas to describe the rigid formation movements by employing the angular momentum concept from classical mechanics. Finally, we explain how the idea can be used for steering a rigid formation to move as a rigid body.

\subsection{Introduction}

\subsubsection{Background and related work}

The stabilization control of multi-agent rigid formation shapes is a typical distributed and cooperative task, in which each agent pair associated with one prescribed interagent distance needs to work cooperatively to achieve that desired distance. This cooperative task requires that agent pairs should have the same view of the desired distance and need to measure consistently, if not correctly, the actual distance between them. The accuracy in measuring some key variables is crucial for achieving the desired formations. In many cases if sensors are located on each agent, they may produce measurement errors or biases, which may result in discrepancies between the estimates of the same actual distance. This problem is also equivalent to one arising when one or more agent pairs may have differing views of the desired inter- 
agent distance that they are tasked to maintain. We use the word mismatch, to refer to the inconsistence of the desired distances between any two adjacent (i.e. neighboring) agents, or the occurrence of differing systematic biases between the actual distances and the measured distances.

It has been briefly mentioned in [Baillieul and Suri, 2003] that such distance mismatches may lead to formation control failure. The concept information-based instability was introduced in [Baillieul and Suri, 2003] to illustrate such a control scenario with conflicting interpretation of information arising in distributed control; see also the review in [Baillieul and Antsaklis, 2007]. Recently, the papers [Belabbas et al., 2012; Sun et al., 2013; Helmke et al., 2014; Mou et al., 2016] have presented more elaborate discussions on these robustness issues in the context of undirected rigid formation shape control in the presence of distance mismatches. It has been shown that the formation shape will converge but additional motions will occur due to mismatched distances. Certain interesting formation movements for 2-D triangular formations and 3-D tetrahedral formations have been discussed in [Belabbas et al., 2012] and [Sun et al., 2013], respectively. In this chapter, we first show, by extending the exponential stability result obtained in [Belabbas et al., 2012] and [Mou et al., 2016], that a 3-D infinitesimally rigid formation system with distance mismatches will still converge to a rigid one. We then give detailed analysis to show the properties of rigid motions induced by small distance mismatches. In this respect, we further generalize the discussions raised in [Baillieul and Suri, 2003], [Baillieul and Antsaklis, 2007] and address the problem of what form of information-based instability would result for the shape stabilization problem of a 3-D rigid formation.

The first focus of this chapter is on the stability issues of 3-D rigid formations with mismatched distances, which partially extend the results in [Belabbas et al., 2012; Mou et al., 2016; Sun et al., 2013] in several aspects. On the one hand, this chapter focuses on rigid motions in 3-D undirected rigid formations, which can be seen as parallel work to the robustness issue work for 2-D rigid formations discussed in [Belabbas et al., 2012] and [Mou et al., 2016]. Note that the extension from 2-D to 3-D is non-trivial and presents totally different outcomes on the rigid motions induced by mismatched distances. Furthermore, compared with [Belabbas et al., 2012; Mou et al., 2016], we develop new methodologies in this chapter based on rigid body dynamics and elementary differential geometry to explain the properties of such rigid motions. On the other hand, this chapter also generalizes the discussions and results from the preliminary version [Sun et al., 2013] which only considered the tetrahedron formation shape.

The second focus of this chapter is to identify the properties and parameters of the rigid formation movements caused by distance mismatches. We note that this formula derivation method for characterizing rigid motions is applicable for both 2$\mathrm{D}$ formations and 3-D formations, while this issue was not discussed in the parallel work [Mou et al., 2016] on 2-D formations. Collective movement for a formation in a 2-D ambient space as discussed in [Mou et al., 2016] means that in steady state the formation exhibits either circular motion around a fixed point common to all 
agents, or translation with each agent moving at the same velocity ${ }^{1}$. Two examples of such planar collective motions have also been studied in [Sepulchre et al., 2008] (for a different problem) in which agents are assumed to have constant unit speed. The collective motion in the 3-D space has an additional degree of freedom, and thus a helical motion becomes possible.

A similar collective helical motion (with parallel motion or circular motion as special cases) for a group of unit-speed agents was discussed in some previous papers; see e.g. [Justh and Krishnaprasad, 2005; Scardovi et al., 2008]. However the problem formulation and motion generation mechanism discussed in [Justh and $\mathrm{Kr}-$ ishnaprasad, 2005; Scardovi et al., 2008] are very different to that arising in rigid formation control to be discussed here. These differences include (i) that in contrast to the system model used in [Justh and Krishnaprasad, 2005; Scardovi et al., 2008], we do not assume constant unit speed in agents' kinematics; (ii) that the collective helical motion discussed here must be consistent with the existence of a rigid formation shape; and (iii) that the rigid motion discussed in this chapter is caused by distance mismatches.

Also, the results in this chapter indicate one interesting mechanism on how to generate rigid motions with specified rigid formation shapes, which may have potential applications for controlling and generating rigid motions for undirected rigid formations with inter-agent distance constraints. We also note that in the literature, helical and spiral motions have been considered as useful motions with particular applications, e.g. for gliding robotic fish [Zhang et al., 2014] and [Zhang et al., 2016] (although the mechanism for generating helical motions discussed in [Zhang et al., 2014] and [Zhang et al., 2016] is different to the result in this chapter).

\subsubsection{Chapter organization}

The remaining parts of this chapter are organized as follows. Section 5.2 presents the problem description and then sets up some key equations of agent motions. In Section 5.3 we focus on the property and convergence of formation shapes, and a relative equilibrium analysis for the additional rigid motion. Section 5.4 shows motion formulas to describe the formation movements in terms of distance mismatches and their applications on steering and controlling rigid formation motions. Section 5.5 concludes this chapter.

\subsection{Motion equations with distance mismatches}

We recall some notations from Chapter 2. Let $d_{k_{i j}}$ denote the desired distance of edge $k$ which links agent $i$ and $j$. The control goal in formation shape stabilization is to drive all the agents to reach a configuration such that a certain set of inter-agent distances can be achieved. We assume that from agent $i$ 's perspective, the specified

\footnotetext{
${ }^{1}$ The research group led by Prof. Ming Cao at the University of Groningen performed several experiments using a group of ground robots to verify such rigid motions in rigid formation control. Videos are available at https://www.youtube.com/user/noeth3r performed by Héctor Garcia de Marina.
} 


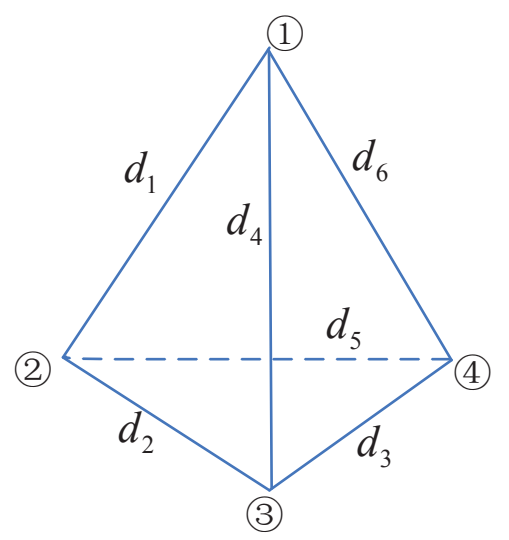

Figure 5.1: An undirected rigid tetrahedron formation.

target distance between agent $i$ and neighbor $j$ is $d_{i j}$ where $d_{i j}$ is a positive number which is approximately equal to $d_{k_{i j}}$. Following the gradient descent control law in Section 2.5 of Chapter 2, we consider the following formation control system in which the control law for agent $i$ is described as ${ }^{2}$

$$
\dot{x}_{i}=u_{i}=\sum_{j \in \mathcal{N}_{i}}\left(x_{j}-x_{i}\right)\left(\left\|x_{j}-x_{i}\right\|^{2}-d_{i j}^{2}\right)
$$

Note that the above gradient control is distributed in the sense that its implementation only requires measurements of relative positions of neighboring agents, denoted by $x_{j}-x_{i}$.

Example: We show an example of a 3-D tetrahedron formation to illustrate the derivation of the system equations described above. Consider a tetrahedron formation in the 3-D space, which consists of four agents labeled by 1,2,3,4. For the purpose of writing an oriented incidence matrix, suppose that the edges are oriented from $i$ to $j$ just when $i<j$. Then we can number the edges in the following order: 12, 23,34,13,24,14; see Figure 5.1. Thus, the following oriented incidence matrix for the undirected graph in Figure 5.1 can be obtained

$$
H=\left[\begin{array}{cccc}
-1 & 1 & 0 & 0 \\
0 & -1 & 1 & 0 \\
0 & 0 & -1 & 1 \\
-1 & 0 & 1 & 0 \\
0 & -1 & 0 & 1 \\
-1 & 0 & 0 & 1
\end{array}\right]
$$

The relative position vector $z$ is then defined according to (2.1) (in Chapter 2). As an example, one has $z_{1}=x_{2}-x_{1}$, i.e., the vector $z_{1}$ at edge 1 is defined by the relative position between agent 2 and agent 1 . Also, from (5.1) one can further obtain

\footnotetext{
${ }^{2}$ Note that in this chapter, we use $x$ instead of $p$ to denote agents' positions. This is for the consistency with the notations used in the paper [Mou et al., 2016] which studied the same topic.
} 
the dynamical system for each agent in the tetrahedron formation control. Again, as an example, the dynamical system for agent 1 can be written as

$$
\dot{x}_{1}=u_{1}=\sum_{j \in \mathcal{N}_{1}}\left(x_{j}-x_{1}\right)\left(\left\|x_{j}-x_{1}\right\|^{2}-d_{1 j}^{2}\right), j=2,3,4 .
$$

and the equations for other agents can be obtained similarly.

Unlike the problem settings in [Krick et al., 2009; Dimarogonas and Johansson, 2010; Cortés, 2009; Dörfler and Francis, 2010; Oh and Ahn, 2014a], we assume in this chapter that the perceived distances $d_{i j}$ and $d_{j i}$ for neighboring agents $i$ and $j$, respectively, are not necessarily equal. The following formulation follows similarly from [Mou et al., 2016]. The distance inconsistency is assumed to satisfy $\left|d_{i j}-d_{j i}\right| \leq$ $\beta_{j i}$ where $\beta_{j i}$ is a small nonnegative number bounding the discrepancy from the two agents' understanding of what the desired distance between them should be. Furthermore, the misbehavior actually stems from the mismatch (the difference, or discrepancy) between $d_{i j}$ and $d_{j i}$ rather than the assumption that both $d_{i j}$ and $d_{j i}$ are only approximately equal to $d_{k_{i j}}$. In other words, only the difference between mutual distances in each edge matters in the modelling of distance mismatch. Without loss of generality and to simplify the equations in the sequel, we will henceforth assume that $d_{i j}$ exactly equals $d_{k_{i j}}$ for all adjacent vertex pairs $(i, j)$ for which $i$ is the head of edge $k_{i j}$. Next, denote $\mu_{k_{i j}}=d_{i j}^{2}-d_{j i}^{2}$ as the constant distance mismatch corresponding to edge $k_{i j}$; clearly, one has

$$
d_{i j}^{2}=d_{k_{i j}}^{2} d_{j i}^{2}=d_{k_{i j}}^{2}-\mu_{k_{i j}}
$$

Let $e_{k_{i j}}$ denote the distance error of the $k$-th edge:

$$
e_{k_{i j}}(z)=\left\|z_{k_{i j}}\right\|^{2}-d_{k_{i j}}^{2}
$$

We denote by $\mathcal{N}_{i}^{+}$the set of all $j \in \mathcal{N}_{i}$ for which vertex $i$ is the head of the oriented edge $k_{i j}$, and denote by $\mathcal{N}_{i}^{-}$the complement of $\mathcal{N}_{i}^{+}$in $\mathcal{N}_{i}$. Then the equation for agent $i^{\prime}$ s motion in the presence of distance mismatch can be written as (see also [Sun et al., 2013; Mou et al., 2016])

$$
\begin{aligned}
\dot{x}_{i} & =-\sum_{j \in \mathcal{N}_{i}}\left(x_{i}-x_{j}\right) e_{k_{i j}}(z) \\
& =-\sum_{j \in \mathcal{N}_{i}^{+}} z_{k_{i j}} e_{k_{i j}}(z)+\sum_{j \in \mathcal{N}_{i}^{-}} z_{k_{i j}}\left(e_{k_{i j}}(z)+\mu_{k_{i j}}\right)
\end{aligned}
$$

where $z_{k_{i j}}$ refers to the $k$ th block entry of the relative position vector $z$ for the edge $k_{i j}$. As noted earlier, for ease of notation we will occasionally use $z_{k_{i j}}$ and $z_{k}$ interchangeably; this will apply to $d_{k_{i j}}$ and $d_{k}, \mu_{k_{i j}}$ and $\mu_{k}, e_{k_{i j}}$ and $e_{k}$ in the following context when the dropping out of the dummy double subscript $i j$ in each vector causes no confusion. The error vector, distance vector and mismatched value vector are constructed as $e=\left[e_{1}, e_{2}, \cdots, e_{m}\right]^{\top}, d=\left[d_{1}, d_{2}, \cdots, d_{m}\right]^{\top}$ and $\mu=\left[\mu_{1}, \mu_{2}, \cdots, \mu_{m}\right]^{\top}$, respectively. In the following, we will use similar techniques as in [Sun et al., 2013] to obtain some 
compact forms of the system equations. First note that the rigidity matrix is given as $R(z)=Z^{\top} \bar{H}$, where $Z=\operatorname{diag}\left\{z_{1}, z_{2}, \cdots, z_{m}\right\}$ (for the derivation, see Section 2.3 in Chapter 2). Define $J$ and $\bar{J}$ to be the matrices obtained from $-H$ and $-\bar{H}$ by replacing all -1 entries by zeros, which also means that $\bar{J}=J \otimes I_{3}$. With the definition of $\bar{J}$, we can define a $m \times 3 n$ matrix $S(z)$ by $S(z)=Z^{\top} \bar{J}$. By doing this, we are led to the following compact equation:

$$
\dot{x}=-R(z)^{\top} e+S^{\top}(z) \mu
$$

which, together with (2.1), implies

$$
\dot{z}=-\bar{H} R^{\top}(z) e(z)+\bar{H} S^{\top}(z) \mu
$$

Note that $\dot{e}=2 R \dot{x}$. In conjunction with (5.6), one obtains

$$
\dot{e}=-2 R(z) R^{\top}(z) e+2 R(z) S^{\top}(z) \mu
$$

In the sequel, we shall refer to (5.6) as the overall system, (5.7) as the $z$ system, and (5.8) as the error system.

Example continued: Following the example of a 3-D tetrahedron formation and the discussions above, we now derive the motion equation for the tetrahedron formation case in the presence of distance mismatches. As an example, the dynamical system for agent 1 in (5.3) with mismatched distances in edges 1, 4 and 6 can be modified as

$$
\begin{gathered}
\dot{x}_{1}=u_{1}=\sum_{j \in \mathcal{N}_{1}}\left(x_{j}-x_{1}\right)\left(\left\|x_{j}-x_{1}\right\|^{2}-d_{1 j}^{2}+\mu_{k}\right), \\
j=2,3,4 ; k=1,4,6 .
\end{gathered}
$$

where the edge index $k=1,4,6$ is associated with adjacent agent pairs $(1,2),(1,3),(1,4)$, respectively; see Figure 5.1. The matrix $J$ in this example can be obtained by replacing all -1 entries of $-H$ in (5.2) by zeros, and the rigidity matrix $R$ and the matrix $S(z)$ can be written as

$$
R(z)=\left[\begin{array}{cccc}
-z_{1} & z_{1} & 0 & 0 \\
0 & -z_{2} & z_{2} & 0 \\
0 & 0 & -z_{3} & z_{3} \\
-z_{4} & 0 & z_{4} & 0 \\
0 & -z_{5} & 0 & z_{5} \\
-z_{6} & 0 & 0 & z_{6}
\end{array}\right], S(z)=\left[\begin{array}{cccc}
z_{1} & 0 & 0 & 0 \\
0 & z_{2} & 0 & 0 \\
0 & 0 & z_{3} & 0 \\
z_{4} & 0 & 0 & 0 \\
0 & z_{5} & 0 & 0 \\
z_{6} & 0 & 0 & 0
\end{array}\right]
$$

By doing this, one can obtain compact equations of system dynamics in the compact form of (5.6) and (5.8), respectively. 


\subsection{Analysis of convergence and formation movements}

\subsubsection{Self-contained distance error equation and exponential convergence}

This subsection aims to show that the distance error system (5.8) for a 3-D formation is self-contained, as is the case for a 2-D formation discussed in [Mou et al., 2016].

Firstly, one can show that the entries of both $R(z) R^{\top}(z)$ and $R(z) S^{\top}(z)$ are linear functions of the entries of the Gramian $\left[z_{1}, z_{2}, \cdots, z_{m}\right]^{\top}\left[z_{1}, z_{2}, \cdots, z_{m}\right]$. By the construction of the relative position vector $z$ as shown in (2.1), it is then obvious that the entries of $\left.R(z) R^{\top}(z)\right|_{z=\bar{H} x}$ and $\left.R(z) S^{\top}(z)\right|_{z=\bar{H} x}$ can be written as a linear combination of inner product terms of the form $\left(x_{i}-x_{j}\right)^{\top}\left(x_{k}-x_{l}\right)$ for $i, j, k, l \in \mathcal{V}$. Let $\{\mathcal{G}, y\}$ be a target formation. Then there exists an open subset $\mathcal{A} \subset \mathbb{R}^{3 n}$ containing $y$ for which the following is true. For each function $f(x)$ defined as $x \mapsto R(z(x)) R^{\top}(z(x))$, there exists a smooth function $\eta_{f}$ with domain $e(\bar{H} \mathcal{A})$ such that $f(x)=\eta_{f}(e(\bar{H} \mathcal{A})), x \in \mathcal{A}$ and the formation $\{\mathcal{G}, x\}$ with $x \in \mathcal{A}$ is infinitesimally rigid and there are values of $x \in \mathcal{A}$ for which $e(x)=0$. A proof for triangular formations and tetrahedral formations can be found in [Belabbas et al., 2012] and [Sun et al., 2013], respectively. The more involved proof for infinitesimally rigid formations with four or more vertices can be found in [Mou et al., 2016]. Note that the proof in [Mou et al., 2016] assumes an underlying 2-D ambient space, but this result can be readily extended to 3-D infinitesimally rigid formations by following the same line of argument, which makes no essential use of the fact that the ambient space is $\mathbb{R}^{2}$ as opposed to $\mathbb{R}^{3}$.

A key step in the convergence analysis is to show that along trajectories of the overall system (5.6), the error system (5.8) satisfies a self-contained differential equation. Let the set $\mathcal{A}$ be the open set as mentioned above. Arguing just as in [Mou et al., 2016], it follows that for the distance error system (5.8), there exists a smooth function $g$ for which $g(e, \mu)=-2 R R^{\top} e+2 R S^{\top} \mu, x \in \mathcal{A}$. Moreover, if $x(t)$ is a solution to the overall system (5.6) for which $x(t) \in \mathcal{A}$ on some interval $\left[t_{0}, t_{1}\right]$, then on the same time interval, the error vector $e=e(\bar{H} x(t))$ satisfies a self-contained differential equation $\dot{e}=g(e, \mu)$. This self-contained error system has an equilibrium close to $e=0$ for each $\mu$ which takes values from a sufficiently small open neighborhood of $\mu=0$ in $\mathbb{R}^{m}$. We refer the readers to [Mou et al., 2016, Section III.A] for a more detailed and rigorous analysis of the above argument. The following results can be seen as direct extensions from the 2-D case to the 3-D case.

Lemma 13. The equilibrium state $e=0$ of the unperturbed error system $\dot{e}=g(e, 0)$ is locally exponentially stable.

Theorem 7. Let $\{\mathcal{G}, y\}$ be a target formation and let $\mathcal{A}$ be the open set referred to above. For each value of $\mu$ in any sufficiently small open neighborhood of $\mu=0$ in $\mathbb{R}^{m}$, and initial state $x(0) \in \mathcal{A}$ for which the error $e(\bar{H} x(0))$ is sufficiently close to the equilibrium $\bar{e}(\mu)$ of the error system $\dot{e}=g(e, \mu)$, the following statements hold:

1. The trajectory of the overall system starting at $x(0)$ exists for all time and lies in $\mathcal{A}$.

2. The error $e=e(\bar{H} x(0))$ converges exponentially fast to $\bar{e}(\mu)$. 
The proofs of Lemma 13 and Theorem 7 are special cases and extensions of the exponential stability result discussed in Chapter 4, obtained by simply choosing the controller function $g_{k}\left(e_{k}\right)=e_{k}$ (see Section 4.2.1 in Chapter 4). We denote the equilibrium of the error system as $\bar{e}(\mu)$, or shortly as $\bar{e}$, which is a continuously differentiable function of $\mu$. Thus, for small $\mu$, all the agents will form a formation shape which is close to the desired one. We also note that, as proved in Chapter 4, the exponential stability still holds for a large family of formation controllers derived from general forms of potential functions, and the following analysis of the robustness issues and motion properties can also be extended to other formation control systems with general controllers. In the sequel, we will still focus on the formation control system (5.6) to present the main results on rigid motion properties.

\subsubsection{Convergence to a rigid formation}

From the convergence of the error vector $e$ to $\bar{e}$, it follows by the argument in Section 5.3.1 that all inner products with the form $\left(x_{i}-x_{j}\right)^{\top}\left(x_{k}-x_{l}\right)$, where $i, j, k, l$ are agent labels, also converge to limits which are continuously differentiable functions of $\bar{e} .^{3}$ Hence the distance between any pair of agents, $i$ and $j$ say, whether or not there is an edge between them, converges to a constant. We summarize the results as below.

Lemma 14. Given the convergence of the distance error $e(t)$ to the equilibrium state $\bar{e}$, the inner product term, $z_{k}^{\top} z_{k}$ for all $k$ and $z_{i}^{\top} z_{j}$ for $i \neq j$, will also converge to constants.

We emphasize here that the convergence of $z_{k}^{\top} z_{k}$ for all $k$ and $z_{i}^{\top} z_{j}$ for $i \neq j$ does not mean that each $z_{k}$ itself converges to a constant. Also, in general the formation will not actually come to rest when the error system converges to $\bar{e}$. We call the formation motion at the equilibrium state $e(\bar{H} x(t))=\bar{e}$ an equilibrium motion. We further denote by $\bar{x}$ and $\bar{z}$ the solutions to the overall system and to the $z$ system, respectively, when the equilibrium state $\bar{e}$ is reached. The study of the dynamics of the $z$ system (5.7) will reveal quite unexpected motions for a mismatched rigid formation, which will be discussed in later sections.

\subsubsection{Rigid motions induced by distance mismatches}

The starting point for the analysis is that all inter-agent distances have reached a steady state, and the formation, given its rigidity property, therefore moves as a rigid body. As such, elementary kinematic principles allow one to define a unique instantaneous angular velocity for the rigid body (and applicable to all points in the rigid body), which indicates how agents move relative to any reference point of the rigid body [Gregory, 2006, Chapter 16].

\footnotetext{
${ }^{3}$ Note that from Section 5.3.1 one concludes that any inner product of the form $z_{i}^{\top} z_{j}$ converges to limits when $e(\bar{H} x(t))=\bar{e}$. Thus due to the infinitesimal rigidity of the target formation, all the inner products with the form $\left(x_{i}-x_{j}\right)^{\top}\left(x_{k}-x_{l}\right)$ will also converge to limits, whether or not there exists an actual edge between agents $i$ and $j$ (or between agents $k$ and $l$ ). A detailed proof for this statement in the 2-D case can be found in [Mou et al., 2016].
} 
In the following analysis we pick the centroid of the formation, denoted as $\bar{x}_{c} \in$ $\mathbb{R}^{3}$ (i.e. $\bar{x}_{c}=\frac{1}{n} \sum_{i=1}^{n} \bar{x}_{i}$ ), as the reference point in the rigid body. Denote by $\bar{r}_{i}$ the vector $\bar{r}_{i}=\bar{x}_{i}-\bar{x}_{c}$, and by $\omega$ the unique instantaneous angular velocity vector of the rigid formation. With this definition and according to rigid body kinematics [Gregory, 2006], one has

$$
\dot{\bar{x}}_{i}=\dot{\bar{x}}_{c}+\omega \times\left(\bar{x}_{i}-\bar{x}_{c}\right)=\dot{\bar{x}}_{c}+\omega \times \bar{r}_{i}
$$

where $\times$ denotes the cross product operation.

We will now show that $\omega$ is constant in the additional motion caused by distance mismatches. We use the fact observed earlier that since $e(\bar{H} x(t))=\bar{e}$, the norm of each relative position vector, $\left\|\bar{z}_{k}\right\|$, and the inner product terms, $\bar{z}_{i}^{\top} \bar{z}_{j}$, are constants. Firstly, we show two results in the following two lemmas dealing with the norm of the velocity for each agent and for the formation centroid.

Lemma 15. The norm of each agent's velocity, i.e. $\left\|\dot{\bar{x}}_{i}\right\|$, is constant when $e(\bar{H} x(t))=\bar{e}$.

Proof. To prove this statement, we rewrite (5.5) by replacing $e$ and $z$ as $\bar{e}$ and $\bar{z}$ at the equilibrium motion:

$$
\dot{\bar{x}}_{i}=-\sum_{j \in \mathcal{N}_{i}^{+}} \bar{z}_{k_{i j}} \bar{e}_{k_{i j}}(z)+\sum_{j \in \mathcal{N}_{i}^{-}} \bar{z}_{k_{i j}}\left(\bar{e}_{k_{i j}}(z)+\mu_{k_{i j}}\right)
$$

One can verify that $\dot{\bar{x}}_{i}^{\top} \dot{\bar{x}}_{i}$ involves the terms of $\mu, \bar{e}, \bar{z}_{k}^{\top} \bar{z}_{k}, \bar{z}_{i}^{\top} \bar{z}_{j}$ for $i \neq j$ and their linear combinations and a certain set of products. According to Lemma 14, these terms are all constant at the equilibrium motion $e(t)=\bar{e}$. These facts lead to $\dot{\bar{x}}_{i}^{\top} \dot{\bar{x}}_{i}$ and $\left\|\dot{\bar{x}}_{i}\right\|$ being constant when $e(t)=\bar{e}$.

Lemma 16. The norm of the formation centroid's velocity, i.e. $\left\|\dot{\bar{x}}_{c}\right\|$, is constant at the equilibrium motion when $e(\bar{H} x(t))=\bar{e}$.

Proof. Since $\dot{\bar{x}}_{c}=\frac{1}{n} \sum_{i=1}^{n} \dot{\bar{x}}_{i}$, we obtain easily the expression of $\dot{\bar{x}}_{c}$ from (5.5):

$$
\dot{\bar{x}}_{c}=\frac{1}{n} \sum_{i=1}^{n} \sum_{j \in \mathcal{N}_{i}^{-}} \bar{z}_{k_{i j}} \mu_{k_{i j}}
$$

Then the same argument as in the proof of the previous lemma applies.

By using the above two lemmas, in the following we will show that $\omega$ is constant at the equilibrium motion. For notational convenience, we introduce a 3 by 3 skewsymmetric matrix $\Omega$ to perform the cross product operation. That is, $\Omega \bar{r}_{i}:=\omega \times \bar{r}_{i}$.

Lemma 17. The angular velocity vector $\omega$ in the 3-D rigid body motions is constant when $e(\bar{H} x(t))=\bar{e}$.

Proof. First observe that

$$
\dot{\bar{z}}_{k_{i j}}=\dot{\bar{x}}_{i}-\dot{\bar{x}}_{j}=\omega \times\left(\bar{r}_{i}-\bar{r}_{j}\right)=\Omega \bar{z}_{k_{i j}}
$$


We recall from (2.1) that the usual expression for the relative position system is $\dot{\bar{z}}=$ $\bar{H} \dot{\bar{x}}$. From the expression of $\dot{\bar{x}}_{i}$ in (5.11), one knows that the expression of $\dot{\bar{z}}_{k_{i j}}$ involves the linear combinations of different edges $\bar{z}_{l}, l \in\{1,2, \cdots, m\}$, with constant weights. Let us consider two arbitrary relative position vectors, say $\bar{z}_{\alpha}$ and $\bar{z}_{\beta}$. From Lemma 14 and the observation just made about the expression of $\dot{\bar{z}}_{k_{i j}}$, it is obvious that $\bar{z}_{\alpha}^{\top} \dot{\bar{z}}_{\beta}=$ constant $=\bar{z}_{\alpha}^{\top} \Omega \bar{z}_{\beta}$. Then one has

$$
\dot{\bar{z}}_{\alpha}^{\top} \Omega \bar{z}_{\beta}+\bar{z}_{\alpha}^{\top} \dot{\Omega}_{\beta}+\bar{z}_{\alpha}^{\top} \Omega \dot{\bar{z}}_{\beta}=0
$$

Note that

$$
\begin{aligned}
\dot{\bar{z}}_{\alpha}^{\top} \Omega \bar{z}_{\beta}+\bar{z}_{\alpha}^{\top} \Omega \dot{z}_{\beta} & =\bar{z}_{\alpha}^{\top} \Omega^{\top} \Omega \bar{z}_{\beta}+\bar{z}_{\alpha}^{\top} \Omega \Omega \bar{z}_{\beta} \\
& =\bar{z}_{\alpha}^{\top}\left(\Omega^{\top} \Omega+\Omega \Omega\right) \bar{z}_{\beta}=0
\end{aligned}
$$

which implies

$$
\bar{z}_{\alpha}^{\top} \dot{\Omega} \bar{z}_{\beta}=0
$$

In any 3-D rigid formations, one can always choose three different vectors $\bar{z}_{\alpha}$ such that they span the $\mathbb{R}^{3}$ space. Similarly, a certain set of $\bar{z}_{\beta}$ can be chosen such that they can also span the $\mathbb{R}^{3}$ space. Hence the only circumstance in which the above equation (5.16) holds for all relative position vectors $\bar{z}_{\alpha}, \bar{z}_{\beta}$ is $\dot{\Omega}=0$, or equivalently, $\Omega$ and $\omega$ are constant.

In the following we further show that the trajectory for each moving agent and the whole rigid formation is in general a helix. In special cases, the rotation-only movement and translation-only movement can also occur, which will be discussed in Section 5.4.

Lemma 18. The motion of each agent, and the motion of the whole rigid formation, will undergo a helical movement.

Proof. We prove the claim of the helical movement by showing that the tangent of each agent's trajectory curve makes a constant angle with a fixed vector, and the fixed vector is in fact the rotational axis $\omega$. To prove the statement in this lemma, it suffices to show

$$
\dot{\bar{x}}_{i}^{\top} \omega=\dot{\bar{x}}_{c}^{\top} \omega+\left(\omega \times \bar{r}_{i}\right)^{\top} \omega=\dot{\bar{x}}_{c}^{\top} \omega=\text { constant }, i=1,2, \cdots, n
$$

By using (5.12), one can restate the above equation (5.17) as

$$
\frac{1}{n}\left(\sum_{i=1}^{n} \sum_{j \in \mathcal{N}_{i}^{-}} \mu_{k_{i j}} \bar{z}_{k_{i j}}\right)^{\top} \omega=\text { constant }
$$

which is equivalent to showing that

$$
\mu_{k_{i j}} \dot{\bar{z}}_{k_{i j}}^{\top} \omega+\mu_{k_{i j}} \bar{z}_{k_{i j}}^{\top} \dot{\omega}=0
$$


To prove that (5.19) holds, first note that $\mu_{k_{i j}} \bar{z}_{k_{i j}}^{\top} \dot{\omega}=0$ because $\dot{\omega}=0$ from Lemma 17. Furthermore, from (5.13) it is obvious that $\dot{\bar{z}}_{k_{i j}}^{\top} \omega=\left(\omega \times\left(\bar{r}_{i}-\bar{r}_{j}\right)\right)^{\top} \omega=0$. Hence (5.19) is proved, and further (5.18) and (5.17) hold.

In [Sun et al., 2013], we proved the helical motion property for a mismatched tetrahedron formation via a long analysis involving the overall system. We note that the above approach for proving the rigid motion property for any mismatched rigid 3-D formation is much simpler and more general.

\subsubsection{Convergence of the equilibrium motion}

This section aims to establish some convergence results based on a relative equilibrium analysis. In the following, we shall rewrite the $z$ system, which was originally stated in (5.7), in another compact form to facilitate the stability analysis. Define $E=\operatorname{diag}\left[e_{1}, e_{2}, \ldots, e_{m}\right], U=\operatorname{diag}\left[\mu_{1}, \mu_{2}, \ldots, \mu_{m}\right]$ and observe that $Z e=\left(E \otimes I_{3}\right) z$ and likewise $Z \mu=\left(U \otimes I_{3}\right) z$. One has

$$
\begin{aligned}
\dot{z} & =-\bar{H} R^{\top}(z) e(z)+\bar{H} S^{\top}(z) \mu \\
& =-\bar{H} \bar{H}^{\top} Z e(z)+\bar{H} \bar{J}^{\top} Z \mu \\
& =-\left(H H^{\top} E \otimes I_{3}\right) z+\left(H J^{\top} U \otimes I_{3}\right) z \\
& =\left(\left(-H H^{\top} E+H J^{\top} U\right) \otimes I_{3}\right) z
\end{aligned}
$$

For ease of notation, we define $F(t):=\left(\left(-H H^{\top} E+H J^{\top} U\right) \otimes I_{3}\right)$. Observe that there holds

$$
\dot{z}=F(t) z \quad \text { and } \quad \dot{\bar{z}}(t)=\left(I_{m} \otimes \Omega\right) \bar{z}(t)
$$

In Section 5.3.3 we have shown that the relative position vector $z_{i}(t)$ in the nonequilibrium system will asymptotically obey the equation $\dot{\bar{z}}_{i}=\omega \times \bar{z}_{i}$ which describes the equilibrium system, when the rigid formation converges exponentially fast to a rigid body. To be precise, we have

$$
M(t):=F(t)-I_{m} \otimes \Omega \rightarrow 0
$$

with convergence at an exponential rate. It is now straightforward to identify a relation between the initial conditions for the two equations in (5.21) that ensures the two solutions approach one another.

Lemma 19. Suppose initial conditions for the equilibrium equation for the relative positions, viz. (5.21), are chosen so that

$$
\bar{z}(0)=z(0)+\int_{0}^{\infty} \exp \left(-\left(I_{m} \otimes \Omega\right) s\right) M(s) z(s) d s
$$

Then $\|z(t)-\bar{z}(t)\| \rightarrow 0$ exponentially fast. 
Proof. Note that because the solution of the non-equilibrium equations is bounded and $M(t)$ is exponentially decaying, the integral in (5.23) is well defined. Let $\tilde{z}(t)=$ $z(t)-\bar{z}(t)$ and observe that

$$
\dot{\tilde{z}}(t)=\left(I_{m} \otimes \Omega\right) \tilde{z}(t)+M(t) z(t)
$$

We shall exhibit exponential convergence to zero of $\tilde{z}(t)$. The solution can be expressed as

$$
\begin{aligned}
\tilde{z}(t)= & \exp \left(\left(I_{m} \otimes \Omega\right) t\right) \tilde{z}(0) \\
& +\int_{0}^{t} \exp \left(\left(I_{m} \otimes \Omega\right)(t-s)\right) M(s) z(s) d s
\end{aligned}
$$

Given the initial condition in (5.23), we see that the above equation can be rewritten as

$$
\tilde{z}(t)=-\int_{t}^{\infty} \exp \left(\left(I_{m} \otimes \Omega\right)(t-s)\right) M(s) z(s) d s
$$

The exponential convergence is immediate, given the boundedness of the trajectory $z(s)$ and the exponential decay of $M(s)$.

The above lemma parallels the non-equilibrium analysis conducted in [Mou et al., 2016] for mismatched 2-D rigid formations. Just as one can carefully select an initial condition to ensure that the non-equilibrium relative position trajectory converges to an equilibrium relative position trajectory, so one can do the same thing for the equations for the overall systems (5.5). This is omitted here.

By combining the results from the above lemmas, we summarize the following theorem which is the second main result of this chapter.

Theorem 8. In the presence of small and constant $\mu$ in the modified distributed gradient control law (5.5), the formation shape converges exponentially fast to a rigid one, and $x(t)$ converges exponentially fast to a helical orbit of the overall system along which $e(\bar{H} x(t))=$ $\bar{e}$.

Example continued: We use numerical simulations on the tetrahedron formation as an example to demonstrate rigid motions induced by distance mismatches. For ease of demonstration we consider a regular tetrahedron shape with the target distance for each edge as 4 . However, there exist small mismatch values in perceived distances (or equivalently in target distances, as defined in (5.4)) for some edges, which are $\mu_{1}=\mu_{2}=0$ (i.e., no distance mismatch in edges 1 and 2), $\mu_{3}=0.05, \mu_{4}=$ $0.05, \mu_{5}=-0.1, \mu_{6}=0.05$. The convergence of the distance errors is depicted in Figure 5.2, which shows that the six distance errors do not actually converge to zero, but converge quickly to some small values very close to zero. This implies that an approximate formation is obtained which is close to the target one. Figure 5.3 shows that all agents quickly form an approximate regular tetrahedron shape and then perform a rigid helical motion as a whole (i.e. moving like a rigid body). 


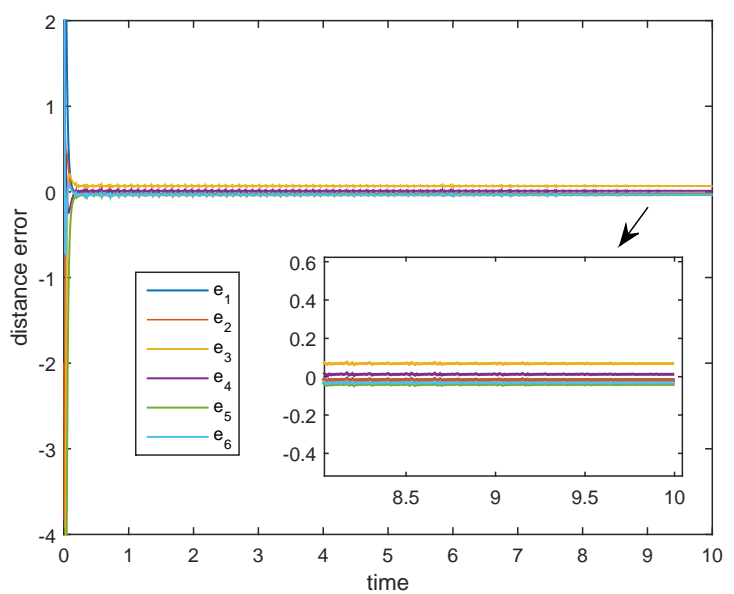

Figure 5.2: Convergence of distance errors.

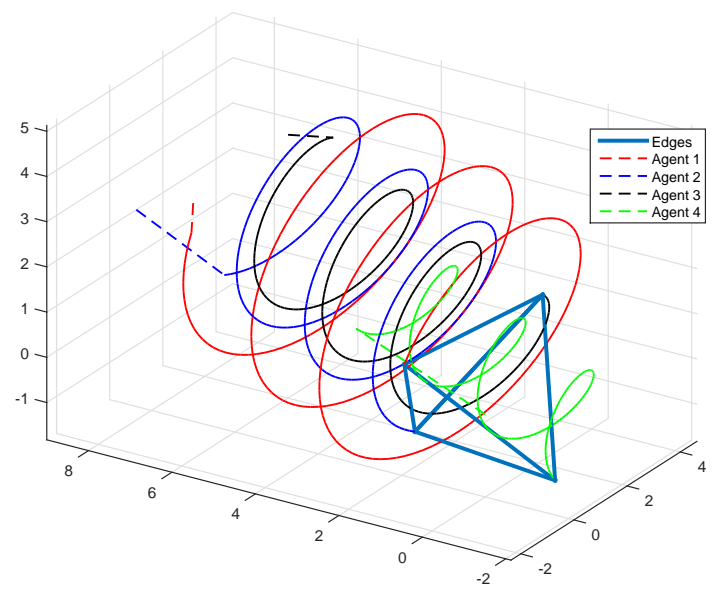

Figure 5.3: Rigid motions (helical motions) induced by distance mismatches in a tetrahedron formation. 


\subsection{Further analysis of the rigid helical motions}

This section aims to provide another perspective of the rigid helical motions caused by distance mismatches. By assuming that $\mu_{k}$ for each edge $k$ is known and constant, we will derive some formulas for the motion parameters including the angular velocity and the rotational radius in terms of $\mu$. The derivation of motion formulas in this section is inspired by the angular momentum concept in rigid body dynamics [Gregory, 2006]. This may have implications in understanding how to actively steer a rigid formation (change of orientation, control of rotation motion, etc) by using a small number of inputs.

\subsubsection{Determining helical motion parameters}

Our starting equation is (5.10). By using basic formulas from rigid body motions involving (5.10) and attributing a unit mass to each agent, the angular momentum of the converged rigid formation measured relative to the formation centroid can be obtained as

$$
\sum_{i=1}^{n} \bar{r}_{i} \times \dot{\bar{x}}_{i}=\sum_{i=1}^{n} \bar{r}_{i} \times \dot{\bar{x}}_{c}+\sum_{i=1}^{n} \bar{r}_{i} \times\left(\omega \times \bar{r}_{i}\right)=\sum_{i=1}^{n} \bar{r}_{i} \times\left(\omega \times \bar{r}_{i}\right)
$$

(Note that $\sum_{i=1}^{n} \bar{r}_{i}=0$ by the definition of the centroid). An important observation is that the left-hand side of (5.27) does not involve the error vector $e$. In fact, one can easily verify using (5.11) that

$$
\sum_{i=1}^{n} \bar{r}_{i} \times \dot{\bar{x}}_{i}=\sum_{i=1}^{n} \sum_{j \in \mathcal{N}_{i}^{-}} \bar{r}_{i} \times \bar{z}_{k_{i j}} \mu_{k_{i j}}
$$

We will examine the last term in the right-hand side of (5.27). To this end we follow a typical approach to define the inertia matrix [Gregory, 2006, Chapter 18] by introducing a skew-symmetric matrix $P_{i}$, which is constructed from the vector $\bar{r}_{i}=\left[\bar{r}_{i, 1}, \bar{r}_{i, 2}, \bar{r}_{i, 3}\right]^{\top}$ :

$$
P_{i}=\left[\begin{array}{ccc}
0 & -\bar{r}_{i, 3} & \bar{r}_{i, 2} \\
\bar{r}_{i, 3} & 0 & -\bar{r}_{i, 1} \\
-\bar{r}_{i, 2} & \bar{r}_{i, 1} & 0
\end{array}\right]
$$

The above skew-symmetric matrix $P_{i}$ is used to perform the cross product operation: $\bar{r}_{i} \times \omega=P_{i} \omega$. Thus one has

$$
\sum_{i=1}^{n} \bar{r}_{i} \times\left(\omega \times \bar{r}_{i}\right)=-\sum_{i=1}^{n} P_{i}\left(\bar{r}_{i} \times \omega\right)=\sum_{i=1}^{n} \mathcal{I}_{i} \omega
$$

where $\mathcal{I}_{i}=P_{i}^{\top} P_{i}$. The following lemma shows the non-singularity of the matrix $\mathcal{I}:=\sum_{i=1}^{n} \mathcal{I}_{i}$.

Lemma 20. The matrix $\mathcal{I}$ is a positive definite symmetric matrix and its inverse exists for generic 3-D rigid formations. 
Proof. The proof is based on the property of the null space of the matrix $P_{i}^{2}=$ $-P_{i}^{\top} P_{i}$. Note that the matrix $P_{i}^{2}$ is negative semidefinite and $\operatorname{null}\left(P_{i}^{2}\right)=\operatorname{null}\left(P_{i}^{\top} P_{i}\right)=$ null $\left(P_{i}\right)$. Note that the null space of the skew symmetric matrix $P_{i}$ is spanned by the vector $\bar{r}_{i}$. Further note that the matrices $P_{i}^{2}$ and $P_{j}^{2}$ do not share the same null space if the vectors $\bar{r}_{i}$ and $\bar{r}_{j}$ are linearly independent. In any generic 3-D rigid formation that can form a rigid body with positive volume, there always exist three linearly independent vectors $\bar{r}_{i}, \bar{r}_{j}, \bar{r}_{k}$ which span the whole 3 -D space. Hence the null space of the matrix $\mathcal{I}$ is trivial, i.e., $\mathcal{I}$ is positive definite and its inverse exists.

By using the above results, the angular velocity at the equilibrium motion can be calculated as

$$
\omega=\mathcal{I}^{-1}\left(\sum_{i=1}^{n} \sum_{j \in \mathcal{N}_{i}^{-}} \bar{r}_{i} \times \bar{z}_{k_{i j}} \mu_{k_{i j}}\right)
$$

which involves the shape geometrical information and mismatch terms $\mu$, but not $e$, as shown in (5.28).

Remark 10. The above equations (5.27) and (5.30) resemble the angular-momentum formula in mechanics, while these two different calculations, (5.27) and (5.30), for the angular momentum are equated. Here each agent can be seen as a particle with unit mass. The left-hand side of (5.27) sums the contribution from each of the point masses to the overall angular momentum, and the right-hand side of (5.30) is the usual expression of the angular momentum involving the inertia matrix $\mathcal{I}$. This provides the interpretation of (5.31) from a physics point of view.

In a helical motion, since the axis along which the agents rotate is also the same direction in which all the agents translate, we can find the translational part of the velocity by projecting the velocity onto the rotational axis. By recalling the velocity for the centroid in (5.12), the translational velocity component can be obtained as $v_{\text {translation }}=\frac{\omega \omega^{\top}}{\omega^{\top} \omega} \dot{\bar{x}}_{c}$ ( and if $\omega=0$, then $v_{\text {translation }}=\dot{\bar{x}}_{c}$ ). The tangential rotational velocity $v_{\text {rotation }}$ and the radius $r_{\text {radius }}^{c}$ of the rotation with respect to the centroid are $v_{\text {rotation }}=\dot{\bar{x}}_{c}-v_{\text {translation, }} r_{\text {radius }}^{c}=\frac{\left\|v_{\text {rotation }}\right\|}{\|\omega\|}$.

Remark 11. (Special motions: translation-only movement) The helical motion also includes the rotation-only movement and translation-only movement as special cases. To guarantee a translation-only movement, the values of $\mu$ should be chosen such that $\omega=0$ which corresponds to requiring $\mu$ to satisfy the following constraints

$$
\sum_{i=1}^{n} \sum_{j \in \mathcal{N}_{i}^{-}} \bar{r}_{i} \times \bar{z}_{k_{i j}} \mu_{k_{i j}}=0
$$

where $\bar{r}_{i}$ and $\bar{z}_{k_{i j}}$ are also smooth functions of $\mu$. It is clear that if $\omega=0$, then $\bar{z}_{k}$ for all $k$ will be constant. By observing that

$$
\begin{aligned}
\bar{r}_{i} \times \bar{z}_{k_{i j}} & =\bar{r}_{i} \times\left(\bar{x}_{j}-\bar{x}_{i}\right)=\bar{r}_{i} \times\left(\left(\bar{x}_{j}-\bar{x}_{c}\right)-\left(\bar{x}_{i}-\bar{x}_{c}\right)\right) \\
& =\bar{r}_{i} \times\left(\bar{r}_{j}-\bar{r}_{i}\right)=\bar{r}_{i} \times \bar{r}_{j}
\end{aligned}
$$


one can see that the condition for translation-only movement shown in (5.32) is equivalent to the statement of Lemma 4 in [Mou et al., 2016]. By using the regular value theorem [Lee, 2009, Proposition 2.42] and following similar arguments as in Lemma 6 of [Mou et al., 2016], one can show that such values of $\mu$ ensuring the special translational motions lie in a thin set.

Remark 12. (Special motions: rotation-only movement) To guarantee a rotation-only movement, the values of $\mu$ should be chosen such that $\omega \neq 0$ and

$$
\dot{\bar{x}}_{c}^{\top} \omega=0
$$

where $\dot{\bar{x}}_{c}$ and $\omega$ depend smoothly on $\mu$ as can be seen from (5.12) and (5.31). To guarantee a rotation-only motion, one needs to either ensure $h(\mu):=\dot{\bar{x}}_{c}^{\top}=0$ (equivalently $\left.\sum_{i=1}^{n} \sum_{j \in \mathcal{N}_{i}^{-}} \bar{z}_{k_{i j}} \mu_{k_{i j}}=0\right)$, or choose values of $\mu$ such that the two vectors $\dot{\bar{x}}_{c} \neq 0$ and $\omega \neq 0$ are orthogonal. By following the same argument as that in Lemma 6 of [Mou et al., 2016], the values of $\mu$ ensuring $\sum_{i=1}^{n} \sum_{j \in \mathcal{N}_{i}^{-}} \bar{z}_{k_{i j}} \mu_{k_{i j}}=0$ can be seen to lie in a thin set, i.e., for generic values of $\mu$ there holds $\dot{\bar{x}}_{c}^{\top} \neq 0$. By inserting the equations from (5.12) and (5.31), the second (orthogonality) condition is rewritten as $h(\mu) \mathcal{I}^{-1}\left(\sum_{i=1}^{n} \sum_{j \in \mathcal{N}_{i}^{-}} \bar{r}_{i} \times \bar{z}_{k_{i j}} \mu_{k_{i j}}\right)=0$. Again, by following the same argument as that in Lemma 6 of [Mou et al., 2016], the non-zero solutions $\mu$ for the above equation can be shown to lie in a thin set. We conclude that such values of $\mu$ ensuring the special translational motions also lie in a thin set. For a simple tetrahedron formation, the conditions for guaranteeing rotation-only or translation-only movement require certain integer-weighted combinations of $\mu$ to be zero; see e.g. [Sun et al., 2013, 2014a].

\subsubsection{Steering formations by manipulating mismatches}

From the above analysis, one can conclude that the final rigid motion with a specific rigid formation shape can be determined by the values of $\mu$. Furthermore, some special choices of $\mu$ are possible which ensure rotation-only movements or translation-only movements. These observations suggest that if indeed values of $\mu$ can be deliberately manipulated, then one can assign different values of $\mu$ to achieve control objectives in relation to formation orientations, angular velocity, translation direction, etc.

We give some brief and intuitive ideas here. In generic 3-D rigid formations, every agent has at least three edges connecting to its neighbors. Let us pick one agent $i$ and its three non-coplanar adjacent relative position vectors, denoted by $\bar{z}_{k_{i 1}}, \bar{z}_{k_{i 2}}, \bar{z}_{k_{i 3}}$ (see Lemma 22 in Chapter 6 for the existence of such vectors). We choose three $\mu_{k_{i 1}}, \mu_{k_{i 2}}, \mu_{k_{i 3}}$ corresponding to these three edges and set all other $\mu_{k}$ in other edges to be zero. Then according to (5.31), one has

$$
\begin{aligned}
\omega & =\mathcal{I}^{-1}\left(\mu_{k_{i 1}} \bar{r}_{i} \times \bar{z}_{k_{i 1}}+\mu_{k_{i 2}} \bar{r}_{i} \times \bar{z}_{k_{i 2}}+\mu_{k_{i 3}} \bar{r}_{i} \times \bar{z}_{k_{i 3}}\right) \\
& =\mathcal{I}^{-1}\left(\bar{r}_{i} \times\left(\mu_{k_{i 1}} \bar{z}_{k_{i 1}}+\mu_{k_{i 2}} \bar{z}_{k_{i 2}}+\mu_{k_{i 3}} \bar{z}_{k_{i 3}}\right)\right)
\end{aligned}
$$

The three vectors $\bar{r}_{i} \times \bar{z}_{k_{i j}}$ where $j=1,2,3$ can be proven to be linearly independent; thus one can always choose the values of three $\mu_{k}$ to achieve any desired vector 
$\omega \in \mathbb{R}^{3}$. A special case is that the sum of the three vectors $\mu_{k_{i j}} \bar{z}_{k_{i j}}$ i.e. $\mu_{k_{i 1}} \bar{z}_{k_{i 1}}+$ $\mu_{k_{i 2}} \bar{z}_{k_{i 2}}+\mu_{k_{i 3}} \bar{z}_{k_{i 3}}$, can be determined to be parallel to the vector $\bar{r}_{i}$, which results in $\omega=0$ and the whole formation will be translating along the direction of $\bar{r}_{i}$ defined in the rigid body coordinates. The same reasoning can also be applied to achieve specific translational and rotational velocities, but we note that because of the linear independence of the three vectors $\bar{r}_{i} \times \bar{z}_{k_{i j}}$, at least four edges will be needed for obtaining a desired vector $\omega$ and a translational velocity. Of course, changing $\mu$ can be expected to change the values of the $\bar{z}_{k_{i j}}$. However, for small $\mu$, these values are approximatable by those of the target formation. Hence a linear equation calculation will deliver values of $\mu$ which at least approximately achieve a desired $\omega$. Similar considerations apply in respect of translation. Thus by using a single agent and its adjacent edges by manipulating mismatches, one can achieve a variety of movements of the formation.

A recent paper [Garcia de Marina et al., 2016a] has provided detailed discussions to explore the application of distance mismatches as motion design parameters to generate desired rigid motions in rigid formation control ${ }^{4}$. We also remark that in [Garcia de Marina et al., 2016a] the formation and motion control can achieve an accurate formation shape (i.e. $e=\bar{e}=0$ ) with desired rigid motions, at the price of carefully choosing mismatch parameters in both adjacent agents for each edge.

\subsection{Concluding remarks}

The popular gradient descent law for stabilizing rigid formation shapes is a typical distributed control approach which requires that neighboring agents should share non-conflicting local information to work cooperatively for a global goal. In this chapter, we have shown some unexpected motion behaviors for this gradient flow when there exist distance mismatches between neighboring agent pairs. We have examined in detail the motion behavior in the 3-D rigid formation shape control problem in the presence of distance mismatches when the gradient control law is employed. The main result shows that in general the formation trajectory at the steady state is a helix, which is a combination of a rotation movement with fixed rotational vector and a translation movement in the direction parallel to that vector. We further show movement properties and formulas to characterize such rigid motions caused by distance mismatches, which reveals an interesting mechanism for generating desired formation movements.

In the case that such rigid motions are regarded as undesirable in formation control, the standard gradient control law should be combined with additional mitigating approaches, e.g. introduction of integral control or inclusion of an estimation loop, to secure a robust formation system. Some recent efforts toward this direction have been proposed in [Mou et al., 2014; Garcia de Marina et al., 2015].

\footnotetext{
${ }^{4}$ Intuitive explanation on how this works can be found in a news report from University of Groningen titled Sensor noise guides flexible robots; see http://www.rug.nl/sciencelinx/nieuws/2016/06/ 20160621_robots.
} 
$72 \quad$ Robustness issues for 3-D undirected formations with distance mismatches 


\section{Distributed stabilization control of rigid formations with prescribed orientations}

\section{Chapter summary}

Most rigid formation controllers reported in the literature aim to only stabilize a rigid formation shape, while the formation orientation is not controlled. This chapter studies the problem of controlling rigid formations with prescribed orientations in both 2-D and 3-D spaces. The proposed controllers involve the commonly-used gradient descent control for shape stabilization, and an additional term to control the directions of certain relative position vectors associated with certain chosen agents. In this control framework, we show the minimal number of agents which should have knowledge of a global coordinate system (2 agents for a 2-D rigid formation and 3 agents for a 3-D rigid formation), while all other agents do not require any global coordinate knowledge or any coordinate frame alignment to implement the proposed control. The exponential convergence to the desired rigid shape and formation orientation is also proved. Typical simulation examples are shown to support the analysis and performance of the proposed formation controllers.

\subsection{Introduction}

\subsubsection{Background and related work}

Rigid formation control has been discussed extensively in the literature, most of which have focused on the convergence analysis of formation shapes (see e.g. [Krick et al., 2009], [Anderson and Helmke, 2014], [Cortés, 2009], [Dörfler and Francis, 2010], [Oh and Ahn, 2011], [Tian and Wang, 2013], [Cai and De Queiroz, 2015]). Note however that in many applications involving multi-agent coordination, a formation with both a desired shape and a particular orientation is required. For distance-based rigid formation control, the orientation of the final formation is not controlled and actu- 
ally not well defined, ${ }^{1}$ which may limit the practical application of shape controllers discussed in these previous works. In this chapter, we aim to design distributed formation controllers to achieve a desired rigid formation with a prescribed formation orientation.

The stabilization control of rigid formations with a desired orientation was discussed in [Pais et al., 2009] by using tensegrity theory and a projected collinear structure. However, the approach, which is essentially adopted from the displacementbased control framework, requires all the agents to have knowledge of the orientation of a common reference frame. The problem of stabilizing only the orientation of rigid objects subject to distance constraints was studied in [Wang et al., 2011], [Markdahl et al., 2012], by assuming that the rigid shapes have been achieved before applying the orientation control. Thus, the approaches in [Wang et al., 2011] and [Markdahl et al., 2012] cannot be applied to solve the formation stabilization control task in question. In our previous paper [Sun et al., 2014a] we showed a feasible approach to move or re-orient a rigid formation to a desired orientation by introducing distance mismatches; however, such an orientation control approach, which is a by-product of the mismatched formation control problem, indicates that the final formation is slightly distorted compared to the desired formation. Furthermore, the orientation control in [Sun et al., 2014a] also requires global information in terms of all other agents' positions, which is contrary to the aim of using a distributed approach for the formation control task.

In this chapter, we propose feasible and distributed controllers to achieve both rigid shape stabilization and formation orientation control with minimal knowledge of global coordinate orientation for the agent group. The basic idea underlying the controller design is to choose certain agents as orientation agents (definitions will become clear in the context), for which some of the associated relative position vectors should achieve both desired distances and directions specified in the global coordinate frame. We note that a very general control framework for stabilizing an affine formation was recently proposed in [Lin et al., 2016a], in which a strict assumption that the target formation should be globally rigid was imposed to generate a rigid shape with orientation constraints. Such an assumption is not required in the control strategy proposed in this chapter.

Furthermore, by exploring several novel observations concerning the rigidity matrix from graph rigidity theory, we will also prove an exponential convergence to the desired formation shape with specified orientations. Note that the exponential stability implies the robustness property of the proposed formation control system in the presence of small measurement errors or perturbations. ${ }^{2}$ Also note that the

\footnotetext{
${ }^{1}$ We need to distinguish different meanings of orientation in the context of formation control. By regarding a rigid formation as a rigid body, the formation orientation relates to the overall rigid formation. The orientation concept in e.g. [Oh and Ahn, 2014b; Montijano et al., 2014] refers to the orientation of the local coordinate frame for each agent. We will distinguish different meanings by referring explicitly to either formation orientation or coordinate orientation. Another orientation concept refers to the definition of signed area for a closed curve formed by a formation shape with a specific ordering of all agents (e.g. a triangle with positive/negative area). This concept will not be used in this chapter.

${ }^{2}$ The problem of securing robustness given massive measurement errors induced by sensor failure
} 
formation orientation problem discussed here is a stabilization control problem (i.e., the aim is to achieve a static formation with a desired orientation), while a motion generation problem involving rigid formation orientation was discussed in [Garcia de Marina et al., 2016a] with a totally different control architecture.

\subsubsection{Chapter organization}

The remaining parts of this chapter are organized as follows. In Section 6.2, we introduce some results relating to the null space of a rigidity matrix as well as the problem formulation. Section 6.3 provides the main result. Typical simulation results are shown in Section 6.4. Finally, Section 6.5 concludes this chapter. Proofs for some key lemmas are given in the Appendix Section 6.6.

\subsection{Preliminaries and problem setup}

\subsubsection{Rigidity matrix and its null space}

We refer the readers to Section 2.3 of Chapter 2 for background on graph rigidity theory. From Theorem 1 in Chapter 2, one knows that the dimension of the null space of $R(p)$ for an infinitesimally rigid framework $(\mathcal{G}, p)$ in the $d$-dimensional space is $d(d+1) / 2$. The following lemma characterizes the structure of its null space.

Lemma 21. (Null space of the rigidity matrix) Suppose the framework $(\mathcal{G}, p)$ is infinitesimally rigid with the associated rigidity matrix denoted as $R(p)$.

- The $d=2$ case: The null space of $R(p)$ is of dimension 3 and is described as null $(R(p))=$ $\operatorname{span}\left(q_{1}, q_{2}, q_{3}\right)$, where

$$
\begin{aligned}
& q_{1}=\mathbf{1}_{n} \otimes\left[\begin{array}{l}
1 \\
0
\end{array}\right] ; q_{2}=\mathbf{1}_{n} \otimes\left[\begin{array}{l}
0 \\
1
\end{array}\right] ; \\
& q_{3}=\left[\left(K_{3} p_{1}\right)^{\top},\left(K_{3} p_{2}\right)^{\top}, \ldots,\left(K_{3} p_{n}\right)^{\top}\right]^{\top},
\end{aligned}
$$

and the matrix $K_{3}$ is defined as

$$
K_{3}=\left[\begin{array}{cc}
0 & 1 \\
-1 & 0
\end{array}\right]
$$

- The $d=3$ case: The null space of $R(p)$ is of dimension 6 and is described as null $(R(p))=$ $\operatorname{span}\left(q_{1}, q_{2}, q_{3}, q_{4}, q_{5}, q_{6}\right)$, where

$$
\begin{gathered}
q_{1}=\mathbf{1}_{n} \otimes\left[\begin{array}{l}
1 \\
0 \\
0
\end{array}\right] ; q_{2}=\mathbf{1}_{n} \otimes\left[\begin{array}{l}
0 \\
1 \\
0
\end{array}\right] ; q_{3}=\mathbf{1}_{n} \otimes\left[\begin{array}{l}
0 \\
0 \\
1
\end{array}\right] ; \\
q_{i}=\left[\left(K_{i} p_{1}\right)^{\top},\left(K_{i} p_{2}\right)^{\top}, \ldots,\left(K_{i} p_{n}\right)^{\top}\right]^{\top}, i=4,5,6 ;
\end{gathered}
$$

is different, and is discussed in [Yu and Anderson, 2009]. 
and the matrix $K_{i}$ is defined as

$$
K_{4}=\left[\begin{array}{ccc}
0 & 0 & 0 \\
0 & 0 & -1 \\
0 & 1 & 0
\end{array}\right] ; K_{5}=\left[\begin{array}{ccc}
0 & 0 & 1 \\
0 & 0 & 0 \\
-1 & 0 & 0
\end{array}\right] ; K_{6}=\left[\begin{array}{ccc}
0 & -1 & 0 \\
1 & 0 & 0 \\
0 & 0 & 0
\end{array}\right]
$$

Lemma 21 is a reformulation of some well known results on the rigidity matrix discussed in Tay and Whiteley's early paper (see [Tay and Whiteley, 1985, Page 31]). We also note that the structure of the null space of a rigidity matrix for 3-D formations was re-stated in [Zelazo et al., 2015, Theorem 2.16]. The clear structure on the null space analysis, as shown in Lemma 21 , will be particularly helpful in the controller design and the stability analysis, as will be shown in the main part of this chapter.

The infinitesimal rigidity also guarantees the following property for a framework.

Lemma 22. Suppose the framework $(\mathcal{G}, p)$ is infinitesimally rigid. Then for any node $i$, the set of relative position vectors $p_{j}-p_{i}, j \in \mathcal{N}_{i}$ cannot all be collinear (in the 2-D case) or all be coplanar (in the 3-D case).

The proof can be found in the online version of the related paper [Sun et al., 2017]. Lemma 22 will be useful for defining a feasible target formation by choosing some adjacent edges associated with certain agents (which will be discussed in Section 6.3.1).

\subsubsection{Gradient-based formation controller and problem formulation}

We recall the following formation control system from Chapter 2:

$$
\dot{p}_{i}=-\sum_{j \in \mathcal{N}_{i}}\left(\left\|p_{i}-p_{j}\right\|^{2}-d_{k_{i j}}^{2}\right)\left(p_{i}-p_{j}\right), i=1, \ldots, n
$$

As mentioned before, the above control and its variations studied in these previous papers only stabilize a rigid formation shape, while the orientation of the formation is not specified. In this chapter we will consider the problem of how to simultaneously stabilize a rigid shape and achieve a desired orientation for a target formation.

\subsection{Main result}

\subsubsection{Target formation and control framework}

Before describing the controller design, we first discuss how to define a target formation with given inter-agent distances and formation orientation constraints. As mentioned in the above section, the commonly-used gradient-based controller (6.1) does not control the orientation and there are certain degrees of freedom relating to rotations for a converged formation. Intuitively, by regarding the rigid formation as a rigid body and specifying certain directions of some chosen edges in a global coordinate frame, the orientation of the overall rigid formation can be fixed. This 
will be the basic idea in the definition of a target formation and the controller design discussed in the sequel.

For simplifying the controller design and implementation, we choose one agent and a certain number of its neighboring agents as the specified agents to implement the additional orientation control task, with the associated edges between them being assigned with both distance constraints and orientation constraints. We term these agents with the additional orientation control task as orientation agents, and other agents as non-orientation agents. Thus, the target formation is defined with interagent distance constraints applying to all the agents, and orientation constraints only for the chosen edges between orientation agents.

For the convenience in later analysis, we denote $\mathcal{G}_{0}$ as the underlying graph of the orientation control to distinguish it from the underlying graph $\mathcal{G}$ of the formation shape control. If the edge $(i, j)$ associated with agent $i$ and $j$ is chosen in the orientation control in $\mathcal{G}_{0}$, we denote it as $(i, j) \in \mathcal{E}_{0}$. The set of neighboring agents for orientation agent $i$ chosen in the orientation control is defined as $\mathcal{N}_{i}^{o}:=\left\{j \in \mathcal{V}:(i, j) \in \mathcal{E}_{0}\right\}$. Note that $\mathcal{G}_{0}$ includes all vertices as $\mathcal{G}$, but it only contains the edges $\mathcal{E}_{0}$. The desired direction for the relative position vector $p_{j}-p_{i}$ for edge $(i, j) \in \mathcal{E}_{o}$ is described by a given vector $\hat{p}_{j i}$. For later analysis, we also introduce some associated fake position vectors $\hat{p}_{j}, \hat{p}_{i} \in \mathbb{R}^{d}$ that realize the specified relative position vector $\hat{p}_{j i}$, i.e. $\hat{p}_{j i}:=\hat{p}_{j}-\hat{p}_{i} .{ }^{3}$ The relative position constraint should also be consistent with the distance constraint associated with the chosen edge (see Definition 5). Thus, the orientation control is used to additionally stabilize the relative position $p_{j}-p_{i}$ to the desired one $\hat{p}_{j}-\hat{p}_{i}$ with $(i, j) \in \mathcal{E}_{0}$. Due to the rigid body property of a desired rigid formation, the formation orientation can be determined by the directions of a certain set of desired relative position vectors. We show two examples, a 2-D fouragent rectangular formation and a 3-D tetrahedral formation depicted in Figure 6.1, to illustrate the formation control framework.

Note that any two agents associated with one edge can be chosen as orientation agents, and there is no need to design a centralized algorithm for the selection of the orientation agents. To define a target formation with prescribed orientation, one can first choose one agent and then select one of its non-collinear relative position vectors (for 2-D formations) or two of its non-coplanar relative position vectors (for 3-D formations) to specify the desired formation orientation. According to Lemma 22 , such non-collinear or non-coplanar adjacent edges are guaranteed to exist for any agent to define a target formation. To sum up, we give a formal definition of a target formation.

Definition 5. (Target formation) The target formation is defined as $(\mathcal{G}, \tilde{p})$ which satisfies the following constraints

- Distance constraints: $\left\|\tilde{p}_{i}-\tilde{p}_{j}\right\|=d_{k_{i j}}, \forall(i, j) \in \mathcal{E}$;

\footnotetext{
${ }^{3}$ The introduction of such vectors is for the convenience of analysis and for writing a compact form of formation system equations, which will be given later in (6.3). This is a commonly-used approach in the analysis of displacement-based formation control; see e.g. [Mesbahi and Egerstedt, 2010, Chapter 6] and [Oh et al., 2015].
} 


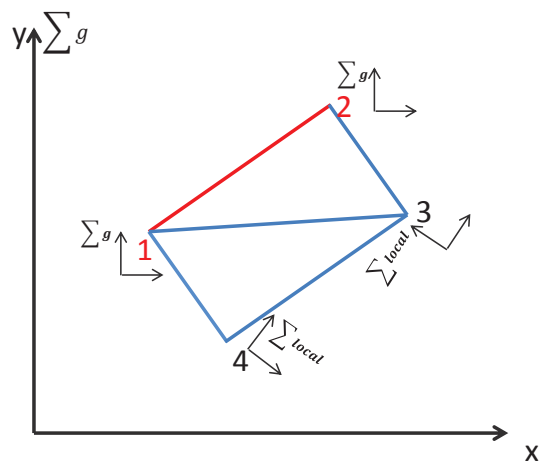

(a)

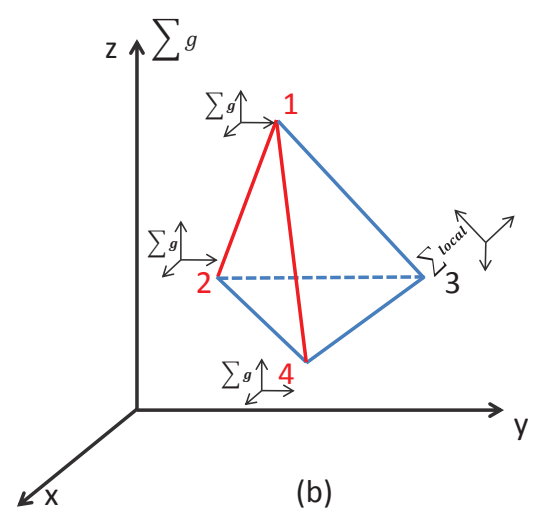

(b)

Figure 6.1: Example of controlling a 2-D rectangular formation and a 3-D tetrahedral formation with prescribed orientation. (a) Agent 1 and one of its neighbors, agent 2, are chosen as orientation agents. The relative position vector $p_{2}-p_{1}$ associated with edge $(1,2)$ is used to describe the desired orientation, which is denoted by red color (in this example $(1,2) \in \mathcal{E}_{0}$ ). (b) Agent 1 and two of its neighbors, agents 2 and 4 , are chosen as orientation agents. The relative position vectors $p_{2}-p_{1}$ and $p_{4}-p_{1}$ associated with edges $(1,2)$ and $(1,4)$ are used to describe the desired orientation, which are denoted by red color (in this example $(1,2),(1,4) \in \mathcal{E}_{0}$ ).

- Orientation constraints: $\tilde{p}_{i}-\tilde{p}_{j}=\hat{p}_{i}-\hat{p}_{j}, \forall(i, j) \in \mathcal{E}_{0}$;

Note that there should hold $\left\|\left(\hat{p}_{j}-\hat{p}_{i}\right)\right\|=d_{k_{i j}}$ so that the orientation constraint is consistent with the formation shape constraint.

In order to well define the orientation constraint, we need the following alignment assumption.

Assumption 1. All orientation agents should be equipped with coordinate systems with the same coordinate axis directions parallel with those of the global coordinate system.

Take the formation control formulation in Figure 6.1(a) as an example. Since agents 1 and 2 are chosen as orientation agents, their coordinate systems should be aligned with the global coordinate system denoted by $\sum_{g}$. Such a global coordinate system is required to define the desired relative position vector $\left(\hat{p}_{j}-\hat{p}_{i}\right)$ for $(i, j) \in \mathcal{E}_{0}$. Thus Assumption 1 provides a necessary condition for the controller design and implementation. ${ }^{4}$

\footnotetext{
${ }^{4}$ We comment that, as shown with more details in [Sun et al., 2017], specification of a single orientation edge does not deal with the problem of reflection ambiguity in determining the formation using a set of prescribed distances.
} 


\subsubsection{Controller design}

We propose the following formation stabilization controller:

$$
\begin{aligned}
\dot{p}_{i}(t)= & \underbrace{\sum_{j \in \mathcal{N}_{i}}\left(p_{j}(t)-p_{i}(t)\right)\left(\left\|p_{j}(t)-p_{i}(t)\right\|^{2}-d_{k_{i j}}^{2}\right)}_{\text {shape control term, if }(i, j) \in \mathcal{E}} \\
& +\underbrace{\sum_{j \in \mathcal{N}_{i}^{o}}\left(\left(p_{j}(t)-p_{i}(t)\right)-\left(\hat{p}_{j}-\hat{p}_{i}\right)\right)}_{\text {orientation control term, if }(i, j) \in \mathcal{E}_{o}}
\end{aligned}
$$

It is obvious from Eq. (6.2) that the proposed control is distributed since only local information from neighboring agents in terms of relative positions is needed. In the later analysis we will also show that the overall system consisting of $n$ agents described by (6.2) is a gradient system associated with a cost function.

The above formation control system (6.2) can be written in a compact form

$$
\dot{p}=-R^{\top} e-\left(L_{o} \otimes I_{d}\right) \bar{p}
$$

where $L_{o}$ is the Laplacian matrix of the underlying undirected graph $\mathcal{G}_{0}$ for the orientation control, and the vector $\bar{p}=\left[\bar{p}_{1}^{\top}, \bar{p}_{2}^{\top}, \ldots, \bar{p}_{n}^{\top}\right]^{\top}$ is defined as $\bar{p}_{i}=p_{i}-\hat{p}_{i}$ if $i$ is a chosen orientation agent ${ }^{5}$, or $\bar{p}_{i}=\mathbf{0}$ otherwise.

For the formation control system (6.3), the set of the desired equilibria is described as

$$
\mathcal{M}=\left\{p \in \mathbb{R}^{d n} \mid e(p)=\mathbf{0}, p_{i}-p_{j}=\hat{p}_{i}-\hat{p}_{j}, \forall(i, j) \in \mathcal{E}_{0}\right\}
$$

which satisfies the constraints in Definition 5.

Example: We show an example to illustrate the above controller design. Suppose a group of four agents is tasked to achieve a rigid shape, with the additional orientation control assigned to edge $(1,2) \in \mathcal{E}_{0}$, which is illustrated in Figure 6.1(a). The formation control system takes the following form

$$
\begin{aligned}
\dot{p}_{1}= & e_{12}\left(p_{2}-p_{1}\right)+e_{13}\left(p_{3}-p_{1}\right)+e_{14}\left(p_{4}-p_{1}\right) \\
& +\left(p_{2}-p_{1}\right)-\left(\hat{p}_{2}-\hat{p}_{1}\right) \\
\dot{p}_{2}= & e_{12}\left(p_{1}-p_{2}\right)+e_{23}\left(p_{3}-p_{2}\right)+\left(p_{1}-p_{2}\right)-\left(\hat{p}_{1}-\hat{p}_{2}\right) \\
\dot{p}_{3}= & e_{13}\left(p_{1}-p_{3}\right)+e_{23}\left(p_{2}-p_{3}\right)+e_{34}\left(p_{4}-p_{3}\right) \\
\dot{p}_{4}= & e_{14}\left(p_{1}-p_{4}\right)+e_{34}\left(p_{3}-p_{4}\right)
\end{aligned}
$$

(note that in the above equations the subscript notation for $e$ is slightly different to previous sections, in that $e_{i j}$ here denotes the squared distance error associated with the edge $(i, j) \in \mathcal{E})$. The Laplacian matrix $L_{o}$ for the underlying graph of orientation

\footnotetext{
${ }^{5}$ Note that the vector $\bar{p}_{i}$ is not an actual control input as $\hat{p}_{i}$ may not be available for agent $i$ (the actual control term is $\hat{p}_{j i}:=\hat{p}_{j}-\hat{p}_{i}$ ). The introduction of $\bar{p}_{i}$ is for the convenience of analysis and for writing a compact form of the formation system as in (6.3).
} 
control is constructed as

$$
L_{o}=\left[\begin{array}{cccc}
1 & -1 & 0 & 0 \\
-1 & 1 & 0 & 0 \\
0 & 0 & 0 & 0 \\
0 & 0 & 0 & 0
\end{array}\right]
$$

and the vector $\bar{p}$ is constructed as $\bar{p}=\left[\left(p_{1}-\hat{p}_{1}\right)^{\top},\left(p_{2}-\hat{p}_{2}\right)^{\top}, \mathbf{0}^{\top}, \mathbf{0}^{\top}\right]^{\top}$. The formation system (6.5) can then be written in the compact form shown in (6.3).

\subsubsection{Properties of the formation control system}

In the following, we show several properties of the proposed control (6.2).

Lemma 23. The position of the formation centroid is preserved by the above control law (6.2).

The proof can be found in the appendix. Lemma 23 corresponds to the statement of invariant centroid in rigid formation control without orientation control, as proved in e.g. [Krick et al., 2009].

Lemma 24. For all non-orientation agents, their local coordinate systems are sufficient to implement the control law.

The proof is omitted here as it follows similarly to the proof in e.g. [Cao et al., 2011; Oh and Ahn, 2014a]. This controller property has been illustrated in Figure 6.1. In the example shown in Figure 6.1(a), agent 3 or 4 is not an orientation agent and its coordinate system orientation does not need to be aligned with the global coordinate system. As a consequence of Lemma 24, the minimum number of orientation agents is 2 for a 2-D rigid formation and 3 for a 3-D rigid formation. Hence the minimum number of agents requiring knowledge of the global coordinate frame for the multiagent formation group is 2 or 3 , according as the 2-D or 3-D case being considered.

\subsubsection{Convergence analysis}

We will first show the gradient property of the proposed controller and a general result on the convergence.

Theorem 9. The formation control system with the proposed controller (6.2) describes a gradient control system and the formation system converges to the largest invariant set in the set $\mathcal{O}(z)$ defined as

$$
\mathcal{O}(z)=\left\{z \mid R(z)^{\top} e(z)+\left(L_{o} \otimes I_{d}\right) \bar{p}(z)=0\right\}
$$

Proof. We choose the same potential function in (2.9), denoted by $V_{1}$, as the potential 
for the shape control, and the following potential function

$$
\begin{aligned}
V_{2} & =\frac{1}{2} \sum_{(i, j) \in \mathcal{E}_{o}}\left\|\left(p_{j}(t)-p_{i}(t)\right)-\left(\hat{p}_{j}-\hat{p}_{i}\right)\right\|^{2} \\
& =\frac{1}{2} \bar{p}^{\top}\left(L_{o} \otimes I_{d}\right) \bar{p}
\end{aligned}
$$

for the orientation control. The composite potential function is then defined as $V(p)=V_{1}(p)+V_{2}(p)$. Note also that both $V_{1}$ and $V_{2}$ can be regarded as functions of $z$, and we will also write them for later analysis as $V_{1}(z)$ and $V_{2}(z)$, as well as $V(z)$.

The dynamical system for the relative position $z$ is described as

$$
\begin{aligned}
\dot{z} & =\left(H \otimes I_{d}\right) \dot{p} \\
& =-\left(H \otimes I_{d}\right) R^{\top} e-\left(H \otimes I_{d}\right)\left(L_{o} \otimes I_{d}\right) \bar{p}
\end{aligned}
$$

and the distance error system is described by

$$
\dot{e}=2 R \dot{p}=-2 R R^{\top} e-2 R\left(L_{o} \otimes I_{d}\right) \bar{p}
$$

Note that (6.8) is a self-contained system since both $e$ and $\bar{p}$ are expressible as functions of $z$. Note further that the potential functions $V_{1}$ and $V_{2}$ are functions involving only relative position vectors in terms of $z$ and $e$ rather than the absolute position vector $p .{ }^{6}$ Thus, we can write the potential as $V(z)$ for the self-contained $z$ system (6.8). We then calculate the derivative of the potential $V_{1}$ and $V_{2}$ along the trajectories of system (6.8) and (6.9):

$$
\begin{aligned}
\dot{V}_{1} & =\frac{1}{2} e^{\top} \dot{e}=e^{\top}\left(-R R^{\top} e-R\left(L_{o} \otimes I_{d}\right) \bar{p}\right) \\
& =-e^{\top} R R^{\top} e-e^{\top} R\left(L_{o} \otimes I_{d}\right) \bar{p}
\end{aligned}
$$

and

$$
\begin{aligned}
\dot{V}_{2} & =\bar{p}^{\top}\left(L_{o} \otimes I_{d}\right) \dot{\bar{p}} \\
& =\bar{p}^{\top}\left(L_{o} \otimes I_{d}\right)\left(-R^{\top} e-\left(L_{o} \otimes I_{d}\right) \bar{p}\right) \\
& =-\bar{p}^{\top}\left(L_{o} \otimes I_{d}\right) R^{\top} e-\bar{p}^{\top}\left(L_{o} \otimes I_{d}\right)\left(L_{o} \otimes I_{d}\right) \bar{p}
\end{aligned}
$$

where in the second equality we have used the non-trivial result $\left(L_{o} \otimes I_{d}\right) \dot{\bar{p}}=\left(L_{o} \otimes\right.$

\footnotetext{
${ }^{6}$ Also note that the distance error vector $e$ can be written in terms of $z$ according to the definition of $e$ in (2.11).
} 
$\left.I_{d}\right) \dot{p}$. The derivative of $V$ can be computed as

$$
\begin{aligned}
\dot{V}= & \dot{V}_{1}+\dot{V}_{2} \\
= & -e^{\top} R R^{\top} e-e^{\top} R\left(L_{o} \otimes I_{d}\right) \bar{p} \\
& -\bar{p}^{\top}\left(L_{o} \otimes I_{d}\right) R^{\top} e-\bar{p}^{\top}\left(L_{o} \otimes I_{d}\right)\left(L_{o} \otimes I_{d}\right) \bar{p} \\
= & -e^{\top} R R^{\top} e-2 e^{\top} R\left(L_{o} \otimes I_{d}\right) \bar{p} \\
& -\bar{p}^{\top}\left(L_{o} \otimes I_{d}\right)\left(L_{o} \otimes I_{d}\right) \bar{p} \\
= & -\left(R^{\top} e+\left(L_{o} \otimes I_{d}\right) \bar{p}\right)^{\top}\left(R^{\top} e+\left(L_{o} \otimes I_{d}\right) \bar{p}\right) \\
\leq & 0
\end{aligned}
$$

The above derivative calculation thus implies that the formation system (6.2) describes a gradient descent flow for the composite potential $V$. Furthermore, the sub-levels set of the potential $V(z)$ are compact with respect to the self-contained $z$ system (6.8). By the LaSalle Invariance Principle [Khalil, 2002], the solution of the formation system (6.8) converges to the largest invariant set in the set $\mathcal{O}(z)=\{z \mid \dot{V}=0\}$ described in (6.6).

The above Theorem 9 focuses on the convergence of the $z$ system (6.8) and its invariant set property, while the convergence of the $z$ system (6.8) to the invariant set (6.6) does not directly guarantee the convergence of the position system (6.3) to a point. We remark that this stronger convergence result (the convergence of the position system (6.3) to a point) can be shown by using the convergence property of the gradient system (see Theorem 2 of Chapter 2, or [Absil and Kurdyka, 2006]) and the real analyticity of the potential $V(p)$, considered as a function of $p$.

Remark 13. In general, a global picture of the convergence analysis for a rigid formation control system is hard to obtain due to the frequent existence of multiple equilibria corresponding to different formation shapes (see discussions in e.g. [Anderson and Helmke, 2014]). Because the proposed control is a gradient descent law, the set $\mathcal{O}(z)$ also describes the set of equilibrium points for (6.2). One may consider a subset of the equilibrium set $\mathcal{O}(z)$, described by

$$
\overline{\mathcal{O}}(z)=\left\{z \mid R(z)^{\top} e(z)=0,\left(L_{o} \otimes I_{d}\right) \bar{p}=0\right\}
$$

In the equilibrium subset $\overline{\mathcal{O}}(z)$ the orientation(s) of the chosen edge(s) is(are) achieved but the overall rigid formation is only described by the equation $R(z)^{\top} e(z)=0$. If one assumes that the target formation is minimally and infinitesimally rigid, and the formation converges to an equilibrium formation in $\overline{\mathcal{O}}(z)$ with an incorrect shape (i.e. $e \neq 0$ ), then the converged incorrect formation is not infinitesimally rigid since $R(z)$ at that equilibrium point has nonzero left null vectors (i.e. the e vector) and thus loses maximum rank (for a detailed discussion on this issue, see [Sun et al., 2016a, Lemma 4]). Since we assume only infinitesimal rigidity of the target formation, one cannot say much about the formation property in the equilibrium set $\overline{\mathcal{O}}(z)$ or $\mathcal{O}(z)$. 
Note that the desired equilibria set $\mathcal{M}$ is a subset of $\overline{\mathcal{O}}(z)$ (and accordingly a subset of $\mathcal{O}(z)$ ). Similar to most works on rigid formation stabilization, in the following we will focus on local convergence analysis. In particular, we aim to show that the convergence to the target formation with desired distances and the prescribed orientation is exponentially fast. The analysis is based on the linearization technique. We first compute the Jacobian of the vector field in the right-hand side of (6.3) around a desired equilibrium $\tilde{p} \in \mathcal{M}$ :

$$
\begin{aligned}
J_{f} & =\left.\frac{\partial\left(-R^{\top} e-\left(L_{o} \otimes I_{d}\right) \bar{p}\right)}{\partial p}\right|_{p=\tilde{p}} \\
& =-\left.\frac{\partial R^{\top}}{\partial p} e\right|_{p=\tilde{p}}-\left.R^{\top} \frac{\partial e}{\partial p}\right|_{p=\tilde{p}}-\left.\frac{\partial\left(L_{o} \otimes I_{d}\right) \bar{p}}{\partial p}\right|_{p=\tilde{p}} \\
& =-\left.\left(R^{\top} R+\left(L_{o} \otimes I_{d}\right)\right)\right|_{p=\tilde{p}}
\end{aligned}
$$

where $\left.\frac{\partial R^{\top}}{\partial p} e(p)\right|_{p=\tilde{p}}=\mathbf{0}$ due to the fact that $e(p)=\mathbf{0}$ for a point $p$ in the equilibrium set $\mathcal{M}$, and $\left.\frac{\partial e}{\partial p}\right|_{p=\tilde{p}}=R$ according to the definition of the rigidity matrix.

Thus, the linearization equation of (6.3) is described as

$$
\delta \dot{p}=-\left(R^{\top} R+L_{o} \otimes I_{d}\right) \delta p
$$

In the following, we prove that the convergence is exponentially fast.

Theorem 10. Suppose the target formation is infinitesimally rigid and initial positions of all the agents are chosen such that the initial formation is close to the desired formation. With the proposed control law (6.2), the convergence to the correct formation shape and orientation is exponentially fast.

Before giving the proof of the above result, we first show a key lemma on the property of the linearization matrix.

Lemma 25. Suppose the target formation is infinitesimally rigid and the orientation edges are selected according to Section 6.3.1. Then the linearization matrix $\mathcal{F}:=R^{\top} R+L_{o} \otimes I_{d}$ is positive semidefinite and has $d$ zero eigenvalues. Furthermore, there holds $\operatorname{null}(\mathcal{F})=$ $\operatorname{null}\left(H \otimes I_{d}\right)=\operatorname{span}\left(\mathbf{1}_{n} \otimes I_{d}\right)$.

The proof can be found in the appendix.

Proof of Theorem 10 As shown in Lemma 23, the formation centroid is stationary. We construct an orthogonal matrix $Q \in \mathbb{R}^{d n \times d n}$ whose first $d$ rows are $\frac{1}{\sqrt{n}}\left(\mathbf{1}_{n} \otimes I_{d}\right)^{\top}$. With $Q$, one can perform the coordinate transform on $p$ as

$$
\tilde{p}=Q p=\left[\begin{array}{l}
p^{o} \\
p_{r}
\end{array}\right]
$$

where $p^{0}=\sqrt{n} p_{c}$ according to the definition of $p_{c}$ in (6.20) and the structure of $Q$. From Lemma 23, one has $\dot{p}^{0}=\sqrt{n} \dot{p}_{c}=\mathbf{0}$. We also define a reduced transformation 
matrix $Q_{r} \in \mathbb{R}^{d(n-1) \times d n}$, obtained from $Q$ by removing the first $d$ rows. Note that there holds $p=Q^{-1} \tilde{p}=Q^{T} \tilde{p}$ and $p_{r}=Q_{r} p$. For the linearized system (6.15), one can obtain the following coordinate-transformed system

$$
\begin{aligned}
{\left[\begin{array}{c}
\delta \dot{p}^{0} \\
\delta \dot{p}_{r}
\end{array}\right]=Q \dot{\delta} p } & =-Q\left(R^{\top} R+L_{o} \otimes I_{d}\right) \delta p \\
& :=-Q \mathcal{F} Q^{-1} \delta \tilde{p}
\end{aligned}
$$

According to the structure of the matrix $Q$, there holds

$$
\begin{aligned}
& Q \mathcal{F} Q^{-1}=\left[\begin{array}{c}
\frac{1}{\sqrt{n}}\left(\mathbf{1}_{n} \otimes I_{d}\right)^{\top} \\
Q_{r}
\end{array}\right] \mathcal{F}\left[\frac{1}{\sqrt{n}}\left(\mathbf{1}_{n} \otimes I_{d}\right) \quad Q_{r}^{\top}\right] \\
& =\left[\begin{array}{cc}
\mathbf{0} & \mathbf{0} \\
\mathbf{0} & Q_{r} \mathcal{F} Q_{r}^{\top}
\end{array}\right]
\end{aligned}
$$

Therefore,

$$
\begin{aligned}
& \delta \dot{p}^{o}=\mathbf{0} \\
& \delta \dot{p}_{r}=-Q_{r} \mathcal{F} Q_{r}^{\top} \delta p_{r}
\end{aligned}
$$

According to the definition of $Q_{r}$, the range space of $Q_{r}^{\top}$ is the orthogonal complement of the subspace $\operatorname{span}\left(\mathbf{1}_{n} \otimes I_{d}\right)$. This, together with Lemma 25, implies that the linearization matrix $-Q_{r} \mathcal{F} Q_{r}^{\top}$ is negative definite. Thus the convergence to the origin for the system (6.19) is locally exponentially fast. Since the system (6.19) is obtained from the system (6.15) by a linear coordinate transformation described in (6.16), the above statement also implies that the convergence to a point in the desired equilibrium $\mathcal{M}$ for the original system (6.3) is locally exponentially fast ([Khalil, 2002, Theorem 4.13]). For the linearized system, the guaranteed exponential convergence rate obtained in the linearization analysis is $\gamma=\lambda_{\min }\left(Q_{r} \mathcal{F} Q_{r}^{\top}\right)$. Note that by the Courant-Fischer Theorem [Zhang, 2011, Theorem 8.9], the rate $\gamma=\lambda_{\min }\left(Q_{r} \mathcal{F} Q_{r}^{\top}\right)$ is the same as the smallest positive eigenvalue of $\mathcal{F}$.

Remark 14. Here we do not confine the discussion to the minimal rigidity case (which is a commonly-used assumption in most literature on rigid formation control). Also we prove the local exponential convergence if the target formation is infinitesimally rigid (a more relaxed assumption than minimal rigidity). It is obvious from the above proof that choosing different orientation edges indeed directly affects the convergence rate, as shown in the expression of the rate $\gamma$. An interesting future problem is to optimize the selection of orientation edges/agents so as to speed up the convergence rate.

Remark 15. Exponential stability brings about several nice properties such as the robustness to small system perturbations (e.g. measurement errors) (similar research direction on robustness issues in rigid formation control was discussed in [Mou et al., 2016]). Note that the exponential convergence cannot be directly extended to the general convergence to a set 
$\mathcal{O}(z)$ stated in Theorem 9. This is because, as indicated in the proof of Theorem 10, the local exponential convergence to a target formation depends on the maximum rank condition of the rigidity matrix of a target formation, and for other formations defined in the set (6.6) one cannot guarantee that they are infinitesimally rigid.

\subsection{Illustrative examples}

In this section we provide several simulations to show formation behaviors and controller performance of the proposed control. Consider a four-agent formation system, with the desired distances given as $d_{12}^{*}=d_{34}^{*}=3, d_{23}^{*}=d_{14}^{*}=4, d_{13}^{*}=5$ corresponding to a rectangular shape. The initial positions for each agent are chosen as $p_{1}(0)=[0,0]^{\top}, p_{2}(0)=[-1,4]^{\top}, p_{3}(0)=[5,3]^{\top}$ and $p_{4}(0)=[3,0]^{\top}$, so that the initial formation shape is close to the target shape. When the conventional controller (6.1) is used, the trajectories of each agent and the final shape are depicted in Figure 6.2, from which it can be seen that although the desired shape is achieved, the formation orientation is undefined.

We then consider the simulation using the proposed controller (6.2). We suppose the target formation should be the one with the rigid rectangular shape as above, in addition that the relative position vector $p_{2}-p_{1}$ associated to edge $(1,2)$ should be aligned with the direction of the $y$-axis and the relative position vector $p_{4}-p_{1}$ associated with the edge $(1,4)$ should be aligned with the direction of the $x$-axis in the global coordinate (which thus defines the unique formation orientation). The desired relative vector for edge $(1,2)$ is set as $\hat{p}_{2}-\hat{p}_{1}=(0,3)^{\top}$ and the initial positions are chosen as the same as the above simulation setting, which can avoid the reflected formation. The trajectories of each agent and the final shape are depicted in Figure 6.3, which clearly show that the desired formation shape with the correct orientation is achieved and the formation centroid is preserved. The trajectories of each distance error and the orientation error for the edge $(1,2)$ are depicted in Figure 6.4, which shows an exponential convergence to the desired formation shape.

Lastly we show an example of stabilizing a rigid 3-D formation with a desired orientation. The target formation is a tetrahedron, with the desired distances given by $d_{12}^{*}=d_{13}^{*}=d_{14}^{*}=2, d_{23}^{*}=d_{34}^{*}=d_{24}^{*}=2 \sqrt{2}$. The desired orientation is that the edges $(1,3)$ and $(1,4)$ should be aligned with the the $x$-axis and the $z$-axis, respectively, which defines the orientation for the target tetrahedron formation. Following the control strategy in Section 6.3.2, the desired relative position vectors for edges $(1,3)$ and $(1,4)$ are defined as $\hat{p}_{1}-\hat{p}_{2}=(2,0,0)^{\top}$ and $\hat{p}_{1}-\hat{p}_{4}=(0,0,2)^{\top}$, in which agents 1,2 and 4 are chosen as orientation agents. The formation convergence is depicted in Figure 6.5, which shows the successful achievement of the formation control task with both desired rigid shape and formation orientation. 


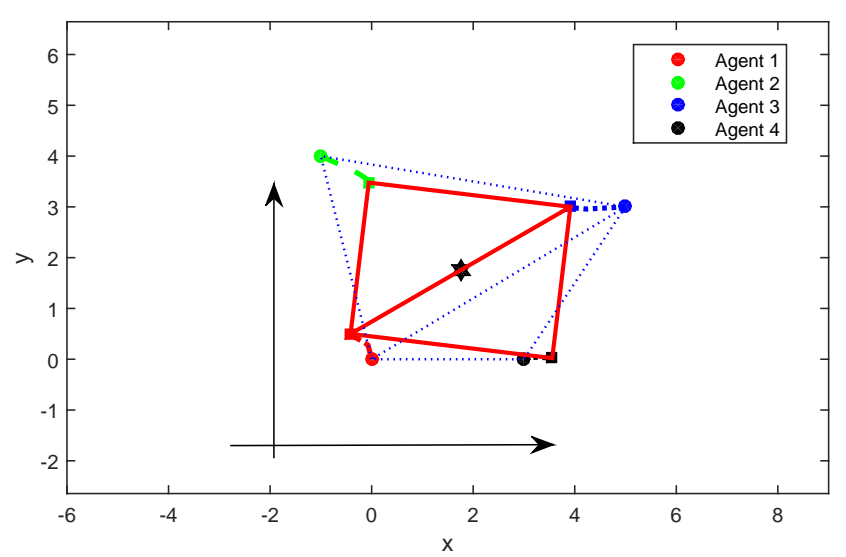

Figure 6.2: Stabilization of a rigid rectangular formation without orientation control. The initial and final positions are denoted by circles and squares, respectively. The initial formation is denoted by dotted blue lines, and the final formation is denoted by red solid lines. The black star denotes the formation centroid.

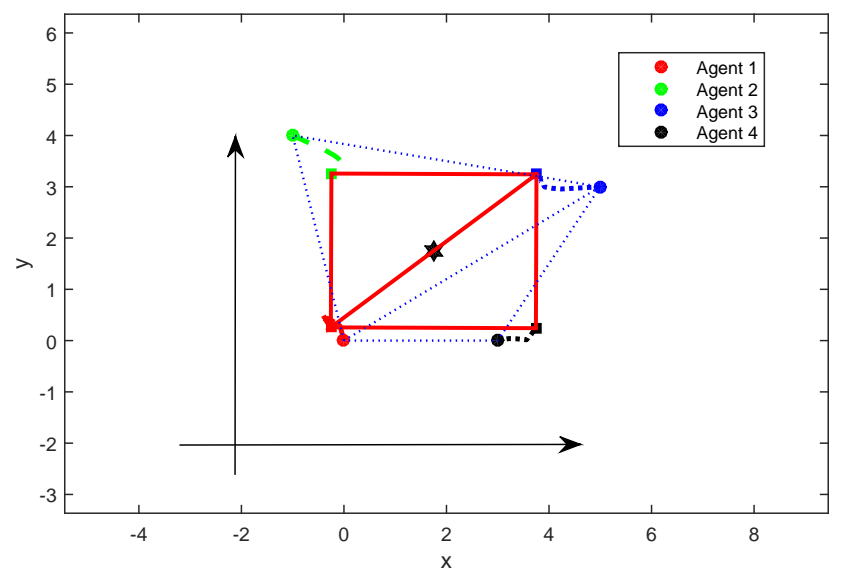

Figure 6.3: Stabilization of a rigid rectangular formation with prescribed orientation. The initial and final positions are denoted by circles and squares, respectively. The initial formation is denoted by dotted blue lines, and the final formation is denoted by red solid lines. The black star denotes the formation centroid. 


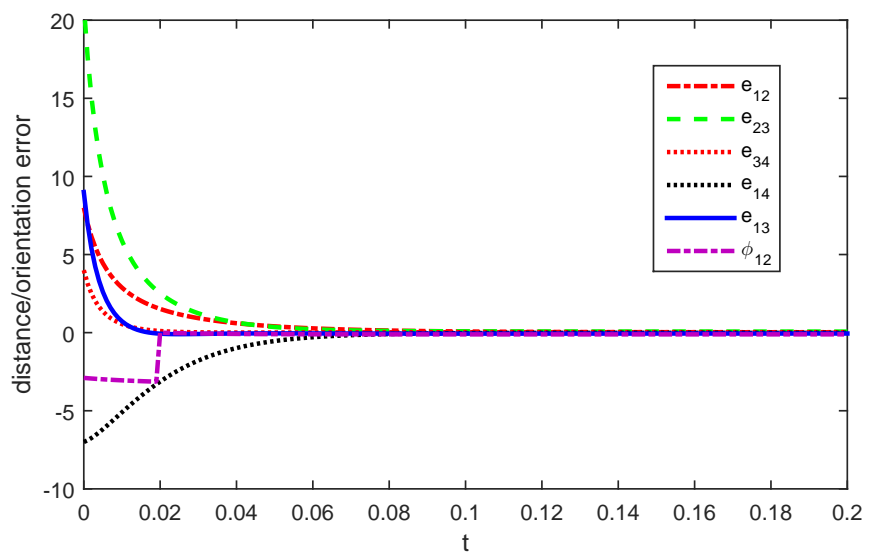

Figure 6.4: Convergence of the distance/orentation errors with the proposed controller (6.2). The orientation error $\phi_{12}$ is defined as $\phi_{12}=\arctan \left(\left(p_{2, y}-p_{1, y}\right) /\left(p_{2, x}-\right.\right.$ $\left.\left.p_{1, x}\right)\right)-\pi / 2$.

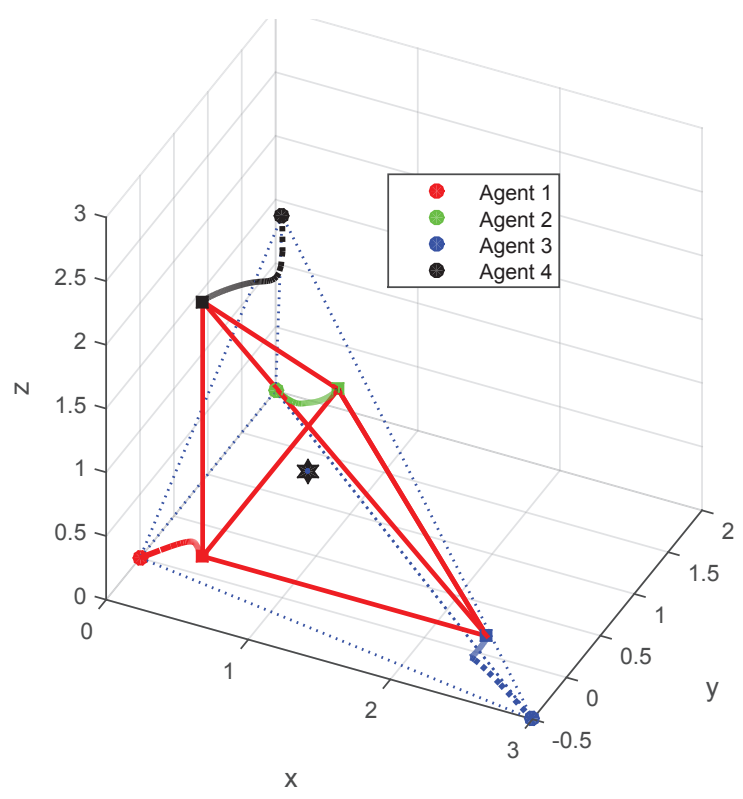

Figure 6.5: Stabilization of a 3-D rigid formation with prescribed orientation. The initial and final positions are denoted by circles and squares, respectively. The initial formation is denoted by dotted blue lines, and the final formation is denoted by red solid lines. The black star denotes the formation centroid. 


\subsection{Concluding remarks}

In this chapter we have discussed the formation control problem to achieve both a desired rigid shape and a formation orientation in ambient 2-D and 3-D spaces. The designed controller combines the advantages of the displacement-based approach and the distance-based approach, by specifying a small number of agents as orientation agents which are tasked to control associated relative position vectors to desired directions. The proposed controllers are distributed in that only relative position measurements from neighboring agents are required. For all non-orientation agents, no information about the global coordinate system is required for them to implement their respective controls, which guarantees a minimal requirement of the global knowledge of the global coordinate system. Certain simulation examples are provided to demonstrate the effectiveness of the proposed formation controllers.

\subsection{Appendix: proofs of several lemmas}

We first show a useful result on the dimension of the null space for two matrices and their product.

Lemma 26. Consider two matrices $A \in \mathbb{R}^{m \times n}$ and $B \in \mathbb{R}^{n \times k}$ and the matrix product $C:=A B$. Then there holds $\operatorname{dim}(\operatorname{null}(C))=\operatorname{dim}(\operatorname{null}(B))+\operatorname{dim}((\operatorname{null}(A) \cap \operatorname{Im}(B))$.

The above lemma can be easily proved via the Sylvester Rank Theorem [Zhang, 2011, Theorem 2.6]. The following corollary is a consequence of Lemma 26 which will be used in the proof of Lemma 25.

Corollary 4. Consider two matrices $A \in \mathbb{R}^{m \times n}$ and $B \in \mathbb{R}^{n \times k}$ and the matrix product $C:=A B$. If $\operatorname{dim}((\operatorname{null}(A) \cap \operatorname{Im}(B))=0$, then null $(C)=\operatorname{null}(B)$.

Proof of Lemma 23 Denote by $p_{c} \in \mathbb{R}^{d}$ the center of the mass of the formation, i.e.,

$$
p_{c}=\frac{1}{n} \sum_{i=1}^{n} p_{i}=\frac{1}{n}\left(\mathbf{1}_{n} \otimes I_{d}\right)^{\top} p
$$

One has

$$
\begin{aligned}
\dot{p}_{c}= & \frac{1}{n}\left(\mathbf{1}_{n} \otimes I_{d}\right)^{\top} \dot{p} \\
= & -\frac{1}{n}\left(\mathbf{1}_{n} \otimes I_{d}\right)^{\top}\left(R^{\top} e+\left(L_{o} \otimes I_{d}\right) \bar{p}\right) \\
= & -\frac{1}{n}\left(\mathbf{1}_{n} \otimes I_{d}\right)^{\top}\left(L_{o} \otimes I_{d}\right) \bar{p} \\
& -\frac{1}{n}\left(Z^{\top}\left(H \otimes I_{d}\right)\left(\mathbf{1}_{n} \otimes I_{d}\right)\right)^{\top} e
\end{aligned}
$$

Note that $\left(\mathbf{1}_{n} \otimes I_{d}\right)^{\top}\left(L_{o} \otimes I_{d}\right) \bar{p}=\left(\left(\mathbf{1}_{n}^{\top} L_{o}\right) \otimes I_{d}\right) \bar{p}=0$ and $\left(Z^{\top}\left(H \otimes I_{d}\right)\left(\mathbf{1}_{n} \otimes I_{d}\right)\right)^{\top} e=$ 0 because null $(H)=\operatorname{span}\left\{\mathbf{1}_{n}\right\}$. Thus $\dot{p}_{c}=0$, which indicates that the position of the formation centroid remains constant. 
Proof of Lemma 25 First note that both $R^{\top} R$ and $L_{o} \otimes I_{d}$ are symmetric and positive semidefinite. From Lemma 21 one knows that $\operatorname{span}\left(\mathbf{1}_{n} \otimes I_{d}\right)$ is a subspace of the null space of $R$. Also, $\operatorname{span}\left(\mathbf{1}_{n} \otimes I_{d}\right)$ is a subspace of the null space of $L_{o} \otimes I_{d}$. Thus, there holds $\operatorname{span}\left(\mathbf{1}_{n} \otimes I_{d}\right) \subset \operatorname{null}(\mathcal{F})$. We then show that there does not exist other null vectors in $\operatorname{null}(\mathcal{F})$.

We introduce a selection matrix, denoted by $J \in \mathbb{R}^{m \times m}$, whose $k$-th row is a row vector with 1 in the $k$-th position and 0 in every other position if the $k$-th edge is selected as an orientation edge, or the $k$-th row is an all-zero vector otherwise. Note that $J^{\top}=J$. Denote the incidence matrix for the underlying graph of orientation control as $H_{o}$. By doing this, there holds $H_{o}=J H$ and thus $L_{o}=H_{o}^{\top} H_{o}=H^{\top} J J H$ since the underlying graph of orientation control is assumed to be undirected. Thus $\mathcal{F}=\left(H \otimes I_{d}\right)^{\top} Z Z^{\top}\left(H \otimes I_{d}\right)+\left(H \otimes I_{d}\right)^{\top}\left(J J \otimes I_{d}\right)\left(H \otimes I_{d}\right)=\left(H \otimes I_{d}\right)^{\top}\left(Z Z^{\top}+J J \otimes\right.$ $\left.I_{d}\right)\left(H \otimes I_{d}\right)$. Following the proof of Lemma 21 (in [Sun et al., 2017]), we define

$$
Q_{j}:=\left[\left(K_{j} z_{1}\right)^{\top},\left(K_{j} z_{2}\right)^{\top}, \cdots,\left(K_{j} z_{m}\right)^{\top}\right]^{\top}
$$

where $j=3$ for the 2-D formation case and $j=4,5,6$ for the 3-D formation case. It is obvious that $Q_{j}$ is a null vector of $Z^{\top}$. Also note that

$$
\begin{aligned}
Q_{j} & =\left(I_{m} \otimes K_{j}\right) z=\left(I_{m} \otimes K_{j}\right)\left(H \otimes I_{d}\right) p \\
& =\left(H \otimes K_{j}\right) p=\left(H \otimes I_{d}\right)\left(I_{n} \otimes K_{j}\right) p
\end{aligned}
$$

We now divide the proof into the following two parts, according to the space dimensions:

- The case of $d=2$ :

From Lemma 21, $Q_{3}$ is a null vector of $Z^{\top}$ and $q_{3}$ is a null vector of $R$. By direct calculation, it holds $\left(Z Z^{\top}+J J \otimes I_{2}\right) Q_{3} \neq 0$, i.e. $Q_{3}$ is not a null vector of the matrix $\left(Z Z^{\top}+J J \otimes I_{2}\right)$, which together with Corollary 4 implies that $q_{3}$ is not a null vector to the matrix $\mathcal{F}$. Thus, there holds $\operatorname{null}(\mathcal{F})=\operatorname{span}\left(\mathbf{1} \otimes I_{2}\right)$, which implies that the null space of $\mathcal{F}$ is of dimension 2 and $\mathcal{F}$ has 2 zero eigenvalues.

- The case of $d=3$ :

We fix $j=4,5,6$. In this case, there are at least two non-zero rows in $J$, corresponding to at least two adjacent edges selected in the orientation control. From Lemma 21, each $Q_{j}$ is a null vector of $Z^{\top}$ and each $q_{j}$ is also a null vector of $R$. Following similar steps as above for the 2-D case and by direct calculation, it holds that $\left(Z Z^{\top}+J J \otimes I_{3}\right) Q_{j} \neq 0$. Thus $Q_{j}{ }^{\prime}$ s are not null vectors of $\left(Z Z^{\top}+J J \otimes I_{3}\right)$, which together with Corollary 4 implies $q_{j}^{\prime}$ s are not null vectors of the matrix $\mathcal{F}$. Thus, there holds $\operatorname{null}(\mathcal{F})=\operatorname{span}\left(\mathbf{1} \otimes I_{3}\right)$, which implies that the null space of $\mathcal{F}$ is of dimension 3 and $\mathcal{F}$ has 3 zero eigenvalues.

By summarizing the above arguments, the statements in the lemma are proved. 


\section{Quantization effects in rigid formation control}

\section{Chapter summary}

In this chapter we discuss quantization effects in rigid formation control systems when target formations are described by inter-agent distances. Because of practical sensing and measurement constraints, we consider in this chapter distance measurements in their quantized forms. We show that under gradient-based formation control, in the case of uniform quantization, the distance errors converge locally to a bounded set whose size depends on the quantization error, while in the case of logarithmic quantization, all distance errors converge locally to zero. A special quantizer involving the signum function is then considered with which all agents can only measure coarse distances in terms of binary information. In this case the formation converges locally to a target formation within a finite time. Lastly, we also discuss the effect of asymmetric uniform quantization on rigid formation control.

\subsection{Introduction}

\subsubsection{Background, motivation and related work}

Quantized control has been an active research topic in the recent decade, motivated by the fact that digital sensors and numerous industrial controllers can only generate quantized measurements or feedback signals [Brockett and Liberzon, 2000; Liberzon, 2003]. Recent years have also witnessed extensive discussions on quantized control for networked control systems. This is because data exchange and transmission over networks often occurs in a digitally quantized manner, thus giving rise to coarse and imperfect information; see e.g., [Kashyap et al., 2007; Cai and Ishii, 2011; Ceragioli et al., 2011; Liu et al., 2012; Frasca, 2012; Guo and Dimarogonas, 2013; Liu et al., 2015].

In this chapter, we aim to discuss the quantization effect on rigid formation control. Formation control based on graph rigidity is a typical networked control problem involving inter-agent measurements and cooperations. There have been many 
papers in the literature focusing on control performance and convergence analysis for rigid formation control systems (see e.g. [Krick et al., 2009; Oh et al., 2015; Sun et al., 2016a]), with virtually all assuming that all agents can acquire the relative position measurements to their neighbors perfectly. We remark that there are some recent works on linear-consensus-based formation control with quantized measurements. An exemplary paper along this line of research is [Jafarian and De Persis, 2015], which showed that by using very coarse measurements (i.e., measurements in terms of binary information) the formation stabilization task can still be achieved. The case of coarse measurements can be seen as a special (or extreme) quantizer, which generates quantized feedbacks in the form of binary signals. However, in [Jafarian and De Persis, 2015] and similar works on linear-consensus-based formation control, a common knowledge of the global coordinate frame orientation is required for all the agents to implement the control law. This is a strict assumption and is not always desirable in practical formation control systems. Actually, it has been shown in [Meng et al., 2016] that coordinate orientation mismatch may also cause undesired formation motions in linear-consensus-based formation systems. All these restrictions and disadvantages can be avoided in rigid formation control systems, in which any common knowledge of the global coordinate system is not required.

In the framework of quantized formation control, we also consider in the latter part of this chapter a special quantizer described by the signum function. This part is motivated by the previous work [Liu et al., 2014] which discussed triangular formation control with coarse distance measurements involving the signum function. In this chapter we will consider a more general setting, which extends the discussions from the triangular case in [Liu et al., 2014] to more general rigid formations.

The aim of this chapter is to explore whether the introduction of quantized measurement and feedback can still guarantee the success of formation control, and to what extent the controller performance limits exist. Our broad conclusion is that quantization is not fatal, but may reduce performance in achieving a target formation.

\subsubsection{Chapter organization}

The remaining parts are organized as follows. Section 7.2 introduces additional notations for this chapter and briefly reviews some background on quantizer functions and non-smooth analysis. Section 7.3 discusses the convergence of the formation systems under two quantized formation controllers. In Section 7.4 we show a special quantized formation controller with binary distance information. Section 7.5 focuses on the case of asymmetric uniform quantizer and its performance. Some illustrative examples are provided in Section 7.6. Section 7.7 concludes this chapter. More tools on non-smooth analysis are provided as appendix in Section 7.8. 


\subsection{Preliminaries}

\subsubsection{Additional notations in this chapter}

In addition to the notations presented in Chapter 2, we now introduce some extra notations that will be solely used throughout this chapter. For a given matrix $A \in$ $\mathbb{R}^{n \times m}$, define $\bar{A}:=A \otimes I_{d \times d} \in \mathbb{R}^{n d \times m d}$, where the symbol $\otimes$ denotes the Kronecker product and $I_{d \times d}$ is the $d$-dimensional identity matrix with $d=\{2,3\}$. We denote by $\|x\|$ the Euclidean norm of a vector $x$, by $\hat{x}:=\frac{x}{\|x\|}$ the unit vector of $x \neq 0$, and by $\tilde{x}:=\frac{1}{\|x\|}$ the reciprocal of the norm of $x \neq 0$. For a stacked vector $x:=$ $\left[x_{1}^{\top}, x_{2}^{\top}, \ldots, x_{k}^{\top}\right]^{\top}$ with $x_{i} \in \mathbb{R}^{l}, i \in\{1, \ldots, k\}$, we define the block diagonal matrix $D_{x}:=\operatorname{diag}\left\{x_{i}\right\}_{i \in\{1, \ldots, k\}} \in \mathbb{R}^{k l \times k}$. The symbol $\operatorname{col}(\cdot)$ defines the column vector by collecting all its arguments as the vector's components.

We define an orientated incidence matrix $B \in \mathbb{R}^{n \times m}$ for the undirected graph $\mathcal{G}$ by assigning an arbitrary orientation for each edge. Note that for a rigid formation modelled by an undirected graph considered here, the orientation of each edge for writing the incidence matrix can be defined arbitrarily and the stability analysis in the next sections remains unchanged. By doing this, we define the entries of $B$ as

$$
b_{i k}:=\left\{\begin{array}{l}
+1, \quad \text { if } \quad i=\mathcal{E}_{k}^{\text {tail }} \\
-1, \quad \text { if } i=\mathcal{E}_{k}^{\text {head }} \\
0, \quad \text { otherwise }
\end{array}\right.
$$

where $\mathcal{E}_{k}^{\text {tail }}$ and $\mathcal{E}_{k}^{\text {head }}$ denote the tail and head nodes, respectively, of the edge $\mathcal{E}_{k}$, i.e. $\mathcal{E}_{k}=\left(\mathcal{E}_{k}^{\text {tail }}, \mathcal{E}_{k}^{\text {head }}\right)$.

\subsubsection{Quantizer functions}

In this chapter, we mainly consider two types of quantizers: the uniform quantizer and the logarithmic quantizer ([Carli et al., 2010; Cai and Ishii, 2011; Frasca, 2012; Guo and Dimarogonas, 2013; Liu et al., 2012; Ceragioli et al., 2011]).

\subsubsection{Definition of the quantizers}

The symmetric uniform quantizer is a map $q_{u}: \mathbb{R} \rightarrow \mathbb{R}$ such that

$$
q_{u}(x)=\delta_{u}\left(\left\lfloor\frac{x}{\delta_{u}}\right\rceil\right)
$$

where $\delta_{u}$ is a positive number and $\lfloor a\rceil, a \in \mathbb{R}$ denotes the nearest integer to $a$. We also define $\left\lfloor\frac{1}{2}+h\right\rceil=h$ for any $h \in \mathbb{Z}$. 


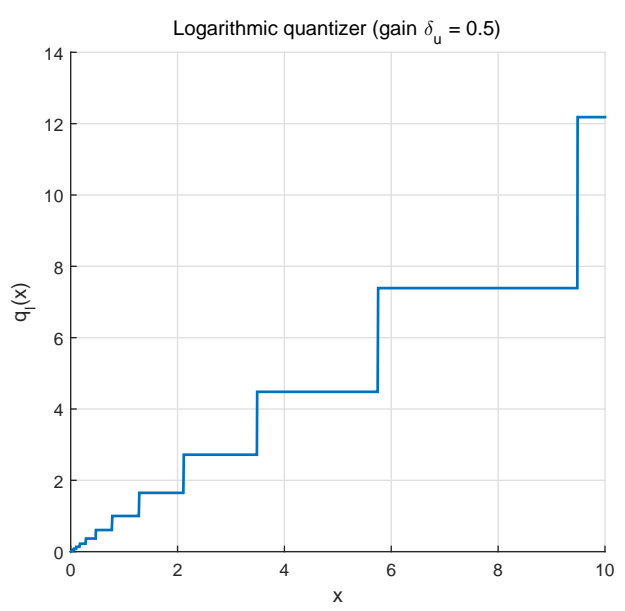

Figure 7.1: Logarithmic quantizer function with the gain $\delta_{u}=0.5$, defined in (7.3).

The logarithmic quantizer is an odd map $q_{l}: \mathbb{R} \rightarrow \mathbb{R}$ such that

$$
q_{l}(x)=\left\{\begin{array}{cc}
\exp \left(q_{u}(\ln x)\right) & \text { when } x>0 \\
0 & \text { when } x=0 \\
-\exp \left(q_{u}(\ln (-x))\right) & \text { when } x<0
\end{array}\right.
$$

where $\exp (\cdot)$ denotes the exponential function.

\subsubsection{Properties of the quantizers}

For the uniform quantizer, the quantization error is always bounded by $\delta_{u} / 2$, namely $\left|q_{u}(x)-x\right| \leq \frac{\delta_{u}}{2}$ for all $x \in \mathbb{R}$.

For the logarithmic quantizer, it holds that $q_{l}(x) x \geq 0$, for all $x \in \mathbb{R}$, and the equality holds if and only if $x=0$. The quantization error for the logarithmic quantizer is bounded by $\left|q_{l}(x)-x\right| \leq \delta_{l}|x|$, where the parameter $\delta_{l}$ is determined by $\delta_{l}=\exp \left(\frac{\delta_{u}}{2}\right)-1$ (note that $\delta_{l}>0$ because $\delta_{u}>0$ ).

The above definitions for scalar-valued uniform and logarithmic quantizers can be generalized to vector-valued quantizers for a vector in a component-wise manner.

For a graph illustration of a logarithmic quantizer function, see Figure 7.1. Note that in Section 7.5 we will further consider an asymmetric uniform quantizer, and will provide some comparisons between symmetric uniform quantizer and asymmetric uniform quantizer. Thus, for the reason of comparisons we will defer the graph illustration of a symmetric uniform quantizer function in Section 7.5 (which is given in Figure 7.2). We also refer the readers to [Carli et al., 2010] for more motivations, comparisons as well as graph illustrations of these two quantizers. 


\subsubsection{Nonsmooth analysis}

Consider a differential equation

$$
\dot{x}(t)=X(x(t)),
$$

where $X: \mathbb{R}^{d} \rightarrow \mathbb{R}^{d}$ is a vector field which is measurable but discontinuous. The existence of a continuously differentiable solution to (7.4) cannot be guaranteed due to the discontinuity of $X(x(t))$. Also, as shown in [Ceragioli et al., 2011], the Caratheodory solutions (for definitions, see [Cortés, 2008]) may not exist from a set of initial conditions of measure zero in quantized control systems. Therefore, we understand the solutions to the quantized rigid formation system in the sense of Filippov ([Filippov, 1988]). We first introduce the Filippov set-valued map.

Definition 6. Let $\mathcal{D}\left(\mathbb{R}^{d}\right)$ denote the collection of all subsets of $\mathbb{R}^{d}$. The Filippov set-valued map $F[X]: \mathbb{R}^{d} \rightarrow \mathcal{D}\left(\mathbb{R}^{d}\right)$ is defined by

$$
\mathcal{F}[X](x) \triangleq \bigcap_{\delta>0} \bigcap_{\mu(\mathcal{S})=0} \overline{\operatorname{co}}\{X(\mathbb{B}(x, \delta) \backslash \mathcal{S})\}, \quad x \in \mathbb{R}^{d}
$$

where $\overline{\mathrm{co}}$ denotes convex closure, $\mathcal{S}$ is the set of $x$ at which $X(x)$ is discontinuous, $\mathrm{B}(x, \delta)$ is the open ball of radius $\delta$ centered at $x$, and $\bigcap_{\mu(\mathcal{S})=0}$ denotes the intersection over all sets $\mathcal{S}$ of Lebesgue measure zero.

Because of the way the Filippov set-valued map is defined, the value of $\mathcal{F}[X]$ at a discontinuous point $x$ is independent of the value of the vector field $X$ at $x$. Filippov solutions are absolutely continuous curves that satisfy almost everywhere the differential inclusion $\dot{x}(t) \in \mathcal{F}[X](x)$ defined above. Some properties of the Filippov solution and examples of how to compute a Filippov set-valued map can be found in the review [Cortés, 2008]. In the appendix, we also list some useful rules relevant to the computation of a Filippov set-valued map.

\subsection{Formation control with quantized measurements}

\section{7·3.1 Quantized formation controllers}

In rigid formation control each agent is required to measure the relative position (i.e. bearing and range) to its neighbors via a bearing sensor and a range sensor. If one assumes perfect measurements, a commonly-used formation controller can be written as (see e.g. [Anderson et al., 2007; Garcia de Marina et al., 2016b] and discussions in Section 4.2.1 of Chapter 4)

$$
\dot{p}_{i}=-\sum_{k=1}^{m} b_{i k}\left(\left\|z_{k}\right\|-d_{k}\right) \hat{z}_{k}
$$

while more general forms of formation controllers to stabilize rigid formations are discussed in Chapter 4 and [Sun et al., 2016a]. 
In the presence of quantized sensing and measurement, the right-hand side of the above formation control system (7.6) needs modification. Here we assume that the distance measurement (with an offset, see the following equation (7.7)) is quantized, and the bearing measurement is unquantized. This assumption is reasonable because the bearing measurement is always bounded (described by a unit vector or by an angle in $[-\pi, \pi)$ in the 2-D case). A normal digital sensor, say a 10-bit uniform quantizer, applying to bearing measurements gives rather accurate measurement with very small error to the true bearing. This is not the case for distance measurements which may have larger magnitudes. We use quantized distance measurement in the formation controller design, while in the future work this may be relaxed by considering both quantized range and bearing measurements. A quantized formation control system can be written as

$$
\dot{p}_{i}=-\sum_{k=1}^{m} b_{i k} q\left(\left\|z_{k}\right\|-d_{k}\right) \hat{z}_{k}
$$

where $q$ is a quantization function, which can be the uniform quantizer or the logarithmic quantizer. We also assume that all the agents use the same quantizer $q(\cdot)$, and their initial positions start with non-collocated positions (i.e. $z_{k}(0) \neq 0$ for all $k$ ).

Remark 16. One may wonder why there is not use of the quantization feedback in the form of $q\left(\| z_{k}||\right)$, i.e. the direct quantized distance measurement, in the control (7.7). We note three reasons for choosing $q\left(\left\|z_{k}\right\|-d_{k}\right)$ instead of $q\left(\| z_{k}||\right)$ :

- In rigid formation control, the control objective is to stabilize the actual distances between neighboring agents to prescribed values. If one chooses the quantization strategy in the form of $q\left(\| z_{k}||\right)$, then this control objective may not be achieved. To this end, the quantization strategy $q\left(\left\|z_{k}\right\|-d_{k}\right)$ used in (7.7) can be interpreted as a digital distance sensor with an embedded or prescribed offset (where the offset is the desired distance $d_{k}$ ).

- In the case of non-uniform quantizers (e.g., logarithmic quantizer), the quantization accuracy usually increases when the quantizer input approaches the desired state (which is the origin in this case). Thus, when the formation approaches closer to the target formation, a higher quantization accuracy (if possible) is required, and this cannot be achieved if one uses the quantization function (e.g., logarithmic quantization) on the actual distance in the form of $q\left(\left\|z_{k}\right\|\right)$.

- We will further show in Section 7.4 that the chosen quantization strategy $q\left(\left\|z_{k}\right\|-\right.$ $d_{k}$ ) will specialize to a simple and effective quantizer with coarse binary distance measurement.

In the presence of quantized measurement and feedback, the right-hand side of (7.7) is discontinuous and we will consider the following differential inclusion

$$
\dot{p}_{i} \in \mathcal{F}\left[\sum_{k=1}^{m} b_{i k} q\left(\left\|z_{k}\right\|-d_{k}\right) \hat{z}_{k}\right] .
$$


In the following, we define the distance error for edge $k$ as $e_{k}=\left\|z_{k}\right\|-d_{k} .{ }^{1}$ We then calculate the differential inclusion $\mathcal{F}\left(q\left(e_{k}\right)\right)$ which will be used in later analysis. In the case of a symmetric uniform quantizer, the differential inclusion $\mathcal{F}\left(q_{u}\left(e_{k}\right)\right)$ can be calculated as

$$
\mathcal{F}\left(q_{u}\left(e_{k}\right)\right)=\left\{\begin{array}{cc}
h \delta_{u}, & e_{k} \in\left(\left(h-\frac{1}{2}\right) \delta_{u},\left(h+\frac{1}{2}\right) \delta_{u}\right), h \in \mathbb{Z} ; \\
{\left[h \delta_{u},(h+1) \delta_{u}\right],} & e_{k}=\left(h+\frac{1}{2}\right) \delta_{u}, h \in \mathbb{Z} .
\end{array}\right.
$$

Note that $e_{k} \mathcal{F}\left(q_{u}\left(e_{k}\right)\right) \geq 0$ for all $e_{k}$, and $e_{k} \mathcal{F}\left(q_{u}\left(e_{k}\right)\right)=0$ if and only if $e_{k} \in\left[-\frac{\delta_{u}}{2}, \frac{\delta_{u}}{2}\right]$.

In the case of a logarithmic quantizer, the differential inclusion $\mathcal{F}\left(q_{l}\left(e_{k}\right)\right)$ can be calculated as

$$
\mathcal{F}\left(q_{l}\left(e_{k}\right)\right)=\left\{\begin{array}{cl}
\operatorname{sign}\left(e_{k}\right) \exp \left(q_{u}\left(\ln \left|e_{k}\right|\right)\right), & e_{k} \neq e^{\left(h+\frac{1}{2}\right) \delta_{u}}, h \in \mathbb{Z} \\
{\left[\exp \left(h \delta_{u}\right), \exp \left((h+1) \delta_{u}\right)\right],} & e_{k}=e^{\left(h+\frac{1}{2}\right) \delta_{u}}, h \in \mathbb{Z} .
\end{array}\right.
$$

Also note that $e_{k} \mathcal{F}\left(q_{l}\left(e_{k}\right)\right)>0$ for all $e_{k} \neq 0$, and $e_{k} \mathcal{F}\left(q_{u}\left(e_{k}\right)\right)=0$ if and only if $e_{k}=0$.

We define the distance error vector as $e=\left[e_{1}, e_{2}, \ldots, e_{m}\right]^{\top}$. Then in a compact form, one can rewrite the dynamics of (7.8) as

$$
\dot{p} \in \mathcal{F}\left[-\bar{B} D_{\hat{z}} q\left(e\left(\underset{k}{\operatorname{col}}\left(\left\|z_{k}\right\|\right)\right)\right] .\right.
$$

In order not to overload the notation, here by $\hat{z}$ we exclusively mean the vector-wise normalization of $z$, therefore $D_{\hat{z}}$ in the above equation and in the sequel is defined as $D_{\hat{z}}=\operatorname{diag}\left\{\hat{z}_{1}, \ldots, \hat{z}_{m}\right\}$. This notation rule will also be applied to $\tilde{z}$ in the sequel. Note that the differential inclusion $\mathcal{F}(q(e))$ with the vector $e$ is defined according to the product rule of Filippov's calculus properties; see Appendix 7.8.

\section{7·3.2 Properties of quantized formation control systems}

We first discuss the solution issue of the formation control system (7.8). However, it is more convenient to focus on the dynamics of the relative position vector $z$, which can be derived from (7.8) as follows

$$
\dot{z}=\bar{B}^{\top} \dot{p} \in \mathcal{F}\left[-\bar{B}^{\top} \bar{B} D_{\hat{z}} q\left(e \underset{k}{\operatorname{col}}\left(\left\|z_{k}\right\|\right)\right)\right] .
$$

First note that at any non-collocated finite initial point $p(0)$, the right-hand side of (7.8) and of (7.10) is measurable and locally essentially bounded. Thus, the existence of a local Filippov solution of (7.8) and of (7.10) starting at such initial points is guaranteed.

We then derive a dynamical system from (7.9) to describe the evolution of the distance error vector $e$. According to the definition of the distance error $e_{k}, e_{k}$ is a smooth function of $z_{k}$. Thus, by using the calculus property shown in (7.30) and

\footnotetext{
${ }^{1}$ Note that the definition of the distance error is different to the one defined in Chapter 2.
} 
Theorem 14 (in the Appendix), one can show $\dot{e}_{k}$ exists and $\dot{e}_{k}=\frac{1}{\left\|z_{k}\right\|} z_{k}^{\top} \dot{z}_{k}$ holds almost everywhere. The dynamics for the distance error vector $e$ can be obtained in a compact form as

$$
\begin{aligned}
\dot{e} & =-D_{\tilde{z}} R(z) \dot{p}, \text { a. e. } \\
& \in-\mathcal{F}\left[D_{\tilde{z}} R(z) R^{\top}(z) D_{\tilde{z}} q(e)\right], \text { a. e. }
\end{aligned}
$$

Again, the existence of a local Filippov solution of (7.11) starting with a non-collocated finite initial point $p(0)$ is guaranteed. In the next section, we will also show that the solutions to (7.11) (as well as the solutions to (7.8) and (7.10)) are bounded and can be extended to $t \rightarrow \infty$ when agents' initial positions are chosen non-collocated and close to a target formation shape. Also, as shown in Lemma 2 in Chapter 2, when the formation shape is close to the desired one, the entries of the matrix $R(z) R^{\top}(z)$ are continuously differentiable functions of $e$. Since the nonzero entries of the diagonal matrix $D_{\tilde{z}}$ are of the form $\frac{1}{\left\|z_{k}\right\|}$ which are also continuously differentiable functions of $e$, we conclude that the system described in (7.11) is a self-contained system, and we will call it the distance error system in the sequel.

Finally, we show some additional properties of the formation control system with quantized information. Note that through this chapter we assume that the underlying graph modelling inter-agent interactions is undirected.

Lemma 27. In the presence of the uniform/logarithmic quantizer, the formation centroid remains stationary.

Proof. Denote by $p_{c} \in \mathbb{R}^{d}$ the center of the mass of the formation, i.e., $p_{c}=\frac{1}{n} \sum_{i=1}^{n} p_{i}=$ $\frac{1}{n}\left(\mathbf{1}_{n} \otimes I_{d \times d}\right)^{\top} p$. By applying the calculus property shown in (7.30) (in the Appendix), one has

$$
\begin{aligned}
\dot{p}_{c} & =\frac{1}{n}\left(\mathbf{1}_{n} \otimes I_{d \times d}\right)^{\top} \dot{p} \\
& \in-\frac{1}{n}\left(\mathbf{1}_{n} \otimes I_{d \times d}\right)^{\top} R^{\top}(z) D_{z} \mathcal{F}[q(e(z))] \text { for a.e. } t
\end{aligned}
$$

Note that $\left(\mathbf{1}_{n} \otimes I_{d \times d}\right)^{\top} R^{\top}(z)=\mathbf{0}$. Therefore,

$$
\dot{p}_{c} \in-\frac{1}{n}\left(\mathbf{1}_{n} \otimes I_{d \times d}\right)^{\top} R^{\top}(z) D_{\tilde{z}} \mathcal{F}[q(e(z))]=\{0\} \text { for a.e. } t .
$$

Thus $\dot{p}_{c}=0$ for a.e. $t$, which indicates that the position of the formation centroid remains constant.

Lemma 28. To implement the control, each agent can use its own coordinate system to measure the relative position (quantized distance and unquantized bearing) and a global coordinate system is not involved.

Proof. The proof is omitted but it can be inferred by following similarly the proof in e.g. [Krick et al., 2009], [Sun et al., 2016a] (or the $S E(d)$ invariance principle in [Vasile et al., 2015]) 


\subsubsection{Convergence analysis}

In this section we aim to prove the following convergence result.

Theorem 11. Suppose the target formation is infinitesimally and minimally rigid and the initial formation shape is close to the target formation shape.

- In the case of a uniform quantizer, the formation converges locally to an approximate and static shape defined by the set $F_{a p p r o x}=\left\{e \mid e_{k} \in\left[-\frac{\delta_{u}}{2}, \frac{\delta_{u}}{2}\right], k \in\{1, \ldots, m\}\right\}$;

- In the case of a logarithmic quantizer, the formation converges locally to a static target formation shape.

In the proof we will use the Lyapunov theory of nonsmooth analysis, for which we construct a Lyapunov function candidate as

$$
V(e)=\sum_{k=1}^{m} V_{k}\left(e_{k}\right), \text { with } V_{k}\left(e_{k}\right)=\int_{0}^{e_{k}} q(s) \mathrm{d} s .
$$

Before giving the proof of Theorem 11, we first show some properties of the function $V$ defined in (7.14). For the definition of function regularity in nonsmooth analysis, see Appendix 7.8.

Lemma 29. The function $V$ constructed in (7.14) is positive semidefinite, and is regular everywhere.

Proof. The positive semidefiniteness of $V$ is obvious from the property of the quantization functions $q_{u}$ and $q_{l}$. Note that $V(e)=0$ if and only if $e \in\left\{e \mid e_{k} \in\left[-\frac{\delta_{u}}{2}, \frac{\delta_{u}}{2}\right], k \in\right.$ $\{1, \ldots, m\}\}$ for a uniform quantizer $q_{u}$, or when $e=0$ for a logarithmic quantizer $q_{l}$. The proof for the regularity is quite long; it is omitted here but follows similarly to the proof of [Liu et al., 2012, Lemma 6].

An observation that supports the regularity statement of $V$ is that $V$ is continuously differentiable almost everywhere, while at the nondifferentiable points $V$ has corners of convex type (see discussions in [Clarke, 2013, Page 200]). Furthermore, according to the definition of generalized derivative in the appendix Section 7.8, one can calculate the generalized derivative of $V_{k}$ (for the case of a uniform quantizer) as

$$
\partial V_{k}=\left\{\begin{array}{cc}
{\left[h \delta_{u},(h+1) \delta_{u}\right],} & e_{k}=\left(h+\frac{1}{2}\right) \delta_{u}, h \in \mathbb{Z} \\
q\left(e_{k}\right), & \text { elsewise }
\end{array}\right.
$$

Similarly, one can also calculate the generalized derivative of $V_{k}\left(e_{k}\right)$ for the case of a logarithmic quantizer (which is omitted here). The generalized derivative of $V(e)$ can be obtained by the product rule (see [Cortés, 2008]). Now we are ready to prove Theorem 11.

Proof. We choose the Lyapunov function constructed in (7.14) for the distance error system (7.11) with discontinuous right-hand side. Note that $R(z) R^{\top}(z)$ and $D_{z}$ are 
positive definite matrices at the desired formation shape. Similarly to the analysis in [Sun et al., 2016a] (or in [Garcia de Marina et al., 2016b]), we define a sub-level set $\mathcal{B}(\rho)=\{e: V(e) \leq \rho\}$ for some suitably small $\rho$, such that when $e \in \mathcal{B}(\rho)$ the formation is infinitesimally minimally rigid, and $R(z) R^{\top}(z)$ and $D_{\tilde{z}}$ are positive definite. Note that the defined sub-level set $\mathcal{B}(\rho)$ is compact. Note also that the matrix $Q(e):=D_{\tilde{z}} R(z) R^{\top}(z) D_{\tilde{z}}$ is also positive definite when $e \in \mathcal{B}(\rho)$, and we rewrite the distance error system as $\dot{e} \in \mathcal{F}[-Q(e) q(e)]$.

The regularity of $V$ shown in Lemma 29 allows us to employ the nonsmooth Lyapunov theorem (shown in Appendix 7.8) to develop the stability analysis. We calculate the set-valued derivative of $V$ along the trajectory of the distance error system (7.11). By applying (7.30), one can obtain

$$
\begin{gathered}
\dot{V}(e)_{(7.11)} \in \tilde{\mathcal{L}}_{(7.11)} V(e)=\left\{a \in \mathbb{R} \mid \exists v \in \dot{e}_{(7.11)},\right. \\
\text { such that } \left.\zeta^{\top} v=a, \forall \zeta \in \partial V(e)\right\} .
\end{gathered}
$$

Note that the set $\tilde{\mathcal{L}}_{(7.11)} V(e)$ could be empty, and in this case we adopt the convention that $\max (\varnothing)=-\infty$. When it is not empty, there exists $v \in-Q(e) q(e)$ such that $\zeta^{\top} v=a$ for all $\zeta \in \partial V(e)$. A natural choice of $v$ is to set $v \in-Q(e) \zeta$, with which one can obtain $a=-q^{\top}(e) Q(e) q(e)$. Let $\bar{\lambda}_{\text {min }}$ denote the smallest eigenvalue of $Q(e)$ when $e(p)$ is in the compact set $\mathcal{B}$ (i.e. $\bar{\lambda}_{\text {min }}=\min _{e \in \mathcal{B}} \lambda(Q(e))>0$ ). Then if the set $\tilde{\mathcal{L}}_{(7.11)} V(e)$ is not empty, one can show

$$
\max \left(\tilde{\mathcal{L}}_{(7.11)} V(e)\right) \leq-\bar{\lambda}_{\min } q(e)^{\top} q(e)
$$

and if the set $\tilde{\mathcal{L}}_{(7.11)} V(e)$ is empty, one has $\max \left(\tilde{\mathcal{L}}_{(7.11)} V(e)\right)=-\infty$. Note that both cases imply that $V$ is non-increasing, and consequently the Filippov solution $e(t)$ of (7.11) is bounded. Thus, all solutions to (7.11) (as well as the solutions to (7.8)) are bounded and can be extended to $t=\infty$ (i.e., there is no finite escape time).

We now divide the rest of the proof into two parts, according to different quantizers:

- The case of uniform quantizers: it can be seen that $\max \left(\tilde{\mathcal{L}}_{(7.11)} V(e)\right) \leq 0$ for all $e \in \mathcal{B}(\rho)$ and $0 \in \max \left(\tilde{\mathcal{L}}_{(7.11)} V(e)\right)$ if and only if $e \in F_{\text {approx. }}$. Also note that $F_{\text {approx }}$ is compact, and is positively invariant for the distance error system (7.11) (i.e. if the initial formation is such that $e(0) \in F_{\text {approx }}$ then all agents are static and $e(t) \in F_{\text {approx }}$ for all $t$ ). According to the nonsmooth invariance principle shown in Theorem 15 (in Appendix 7.8), the first part of the convergence result is proved. Since this is a convergence to a bounded set, the convergence is achieved within a finite time. Note from (7.7) the final formation is stationary.

- The case of logarithmic quantizers: it can be seen that $\max \left(\tilde{\mathcal{L}}_{(7.11)} V(e)\right) \leq 0$ for all $e \in \mathcal{B}(\rho)$ and $0 \in \max \left(\tilde{\mathcal{L}}_{(7.11)} V(e)\right)$ if and only if $e=0$. According to the nonsmooth invariance principle shown in Theorem 15, the second part of the convergence result is proved. Also note from (7.7) the final formation is stationary. 
The proof is thus completed.

\subsection{A special quantizer: formation control with binary dis- tance information}

\section{7·4.1 Rigid formation control with coarse measurements}

In this section we consider the special case in which each agent uses very coarse distance measurements, in the sense that it only needs to detect whether the current distance to each of its neighbors is greater or smaller than the desired distance. This gives rise to a special quantizer defined by the following signum function:

$$
\operatorname{sign}(x)=\left\{\begin{array}{cc}
1 & \text { when } x>0 \\
0 & \text { when } x=0 \\
-1 & \text { when } x<0
\end{array}\right.
$$

Accordingly, we obtain the following rigid formation control system with binary distance measurements:

$$
\dot{p}_{i}=-\sum_{k=1}^{m} b_{i k} \operatorname{sign}\left(\left\|z_{k}\right\|-d_{k}\right) \hat{z}_{k}
$$

Remark 17. Formation control using the signum function has been discussed in several previous papers. In [Zhao et al., 2014], a finite-time convergence was established for stabilization of cyclic formations using binary bearing-only measurements. The paper [Liu et al., 2014] studied the stabilization control of a cyclic triangular formation with the controller (7.17). Here we extend such controllers to stabilize general undirected formation which are minimally and infinitesimally rigid. The above controller (7.17) can also be seen as a highdimensional extension of the one-dimensional formation controller studied in [De Persis et al., 2010]. Also, note that the right-hand side of (7.17) is composed of the sum of a unit vector multiplied by a signum function. This implies that the formation controller (7.17) is of special interest in practice since the control action is explicitly upper bounded by the cardinality of the set of neighbors for each agent $i$, which prevents potential implementation problems due to saturation.

Again, we consider the Filippov solution to the formation control system (7.17). The differential inclusion $\mathcal{F}\left(\operatorname{sign}\left(e_{k}\right)\right)$ can be calculated as

$$
\mathcal{F}\left(\operatorname{sign}\left(e_{k}\right)\right)=\left\{\begin{array}{cl}
1 & \left\|z_{k}\right\|>d_{k}, \\
{[-1,1],} & \left\|z_{k}\right\|=d_{k}, \\
-1 & \left\|z_{k}\right\|<d_{k} .
\end{array}\right.
$$

In a compact form, the rigid formation system (7.17) can be rewritten as

$$
\dot{p} \in \mathcal{F}\left[-R^{\top}(z) D_{\tilde{z}} \operatorname{sign}(e)\right],
$$


where $\operatorname{sign}(e)$ is defined in a component-wise way.

Note that the right-hand side of (7.18) is measurable and essentially bounded at any non-collocated and finite point $p$, and the existence of a local Filippov solution to (7.18) is guaranteed from such an initial point $p(0)$. In the following analysis we will also show that the solutions are bounded and complete.

Similar to the analysis in deriving the distance error system shown in Section 7.3.2, the distance error system with binary distance information can be obtained as

$$
\dot{e} \in \mathcal{F}\left[-D_{\tilde{z}} R(z) R^{\top}(z) D_{\tilde{z}} \operatorname{sign}(e)\right], \text { a. e. }
$$

Again, similar to the analysis for (7.11), one can also show that (7.19) is a selfcontained system when $e$ takes values locally around the origin.

\subsubsection{Convergence analysis}

The main result in this section is stated in the following convergence theorem for the formation controller (7.18) with binary distance information.

Theorem 12. Suppose the target formation is infinitesimally and minimally rigid, the initial formation shape is close to the target formation shape, and the formation controller (7.17) with binary distance information is applied.

- The formation converges locally to a static target formation shape;

- The convergence is achieved within a finite time upper bounded by $T^{*}=\frac{\|e(0)\|_{1}}{\bar{\lambda}_{\min }}$ where $\bar{\lambda}_{\min }$ is defined in the proof.

Proof. Part of the proof for this theorem is similar to the proof of Theorem 11. Choose the Lyapunov function defined as $V=\sum_{k=1}^{m} V_{k}\left(e_{k}\right)$ with $V_{k}\left(e_{k}\right)=\left|e_{k}\right|$ for the distance error system (7.19). Note that $V$ is a convex and regular function of $e$. Also $V$ is locally Lipschitz at $e=0$ and is continuously differentiable at all other points. The generalized derivative of $V_{k}\left(e_{k}\right)$ can be calculated as

$$
\partial V_{k}=\left\{\begin{array}{cc}
1, & e_{k}>0 \\
{[-1,1],} & e_{k}=0 \\
-1, & e_{k}<0
\end{array}\right.
$$

and the generalized derivative of $V$ can be calculated similarly via the product rule (see [Cortés, 2008]). We define a sub-level set $\mathcal{B}(\rho)=\{e: V(e) \leq \rho\}$ for some suitably small $\rho$, such that when $e \in \mathcal{B}(\rho)$ the formation is infinitesimally minimally rigid and $R(z) R^{\top}(z)$ and $D_{\tilde{z}}$ are positive definite. Now the matrix $Q(e):=D_{\tilde{z}} R(z) R^{\top}(z) D_{\tilde{z}}$ is also positive definite when $e \in \mathcal{B}(\rho)$. Let $\bar{\lambda}_{\text {min }}$ denote the smallest eigenvalue of $Q(e)$ when $e(p)$ is in the compact set $\mathcal{B}$ (i.e. $\bar{\lambda}_{\min }=\min _{e \in \mathcal{B}} \lambda(Q(e))>0$ ).

In the following, we calculate the set-valued derivative of $V$ along the trajectory described by the differential inclusion (7.19). The argument follows similarly to the 
analysis in the proof of Theorem 11. By applying (7.30), the set-valued derivative is described by

$$
\begin{gathered}
\dot{V}(e)_{(7.19)} \in \tilde{\mathcal{L}}_{(7.19)} V(e)=\left\{a \in \mathbb{R} \mid \exists v \in \dot{e}_{(7.19)},\right. \\
\text { such that } \left.\zeta^{\top} v=a, \forall \zeta \in \partial V(e)\right\} .
\end{gathered}
$$

If the set $\tilde{\mathcal{L}}_{(7.19)} V(e)$ is not empty, there exists $v \in-Q(e) \operatorname{sign}(e)$ such that $\zeta^{\top} v=a$ for all $\zeta \in \partial V(e)$. A natural choice of $v$ is to set $v \in-Q(e) \zeta$, with which one can obtain $a=-\operatorname{sign}^{\top}(e) Q(e) \operatorname{sign}(e)$. Then one can further show

$$
\max \left(\tilde{\mathcal{L}}_{(7.19)} V(e)\right) \leq-\bar{\lambda}_{\min } \operatorname{sign}(e)^{\top} \operatorname{sign}(e),
$$

if the set is not empty, while if it is empty we adopt the convention $\max \left(\tilde{\mathcal{L}}_{(7.19)} V(e)\right)=$ $-\infty$. Note that this implies that $V$ is non-increasing, and consequently the Filippov solution $e(t)$ is bounded. Thus, all solutions to (7.19) (as well as the solutions to (7.18)) are complete and can be extended to $t=\infty$ (i.e., there is no finite escape time). It can be seen that $\max \left(\tilde{\mathcal{L}}_{(7.19)} V(e)\right) \leq 0$ for all $e \in \mathcal{B}(\rho)$ and $0 \in \max \left(\tilde{\mathcal{L}}_{(7.19)} V(e)\right)$ if and only if $e=0$. According to the nonsmooth invariance principle shown in Theorem 15, the asymptotic convergence is proved.

We then prove the stronger convergence result, i.e., the finite-time convergence. From the definition of the sign function in (7.17), there holds $\operatorname{sign}(e)^{\top} \operatorname{sign}(e)>1$ for any $e \neq 0$, which implies

$$
\max \left(\tilde{\mathcal{L}}_{(7.19)} V(e)\right) \leq-\bar{\lambda}_{\min }
$$

for any $e \neq 0$. Thus, by applying Theorem 17 (in the Appendix), any solution starting at $e(0) \in \mathcal{B}(\rho)$ reaches the origin in finite time, and the convergence time is upper bounded by $T^{*}=V(e(0)) / \bar{\lambda}_{\min }=\|e(0)\|_{1} / \bar{\lambda}_{\min }$.

Remark 18. (Dealing with chattering) In the controller (7.17) the sign function is used, which may cause chattering when the formation is very close to the desired one (i.e. when $e$ is very close to the origin). Possible solutions to eliminate the chattering include the following:

- Add deadzone to the sign function around the origin (similar to the case of uniform quantizers; see Part 1 of Theorem 11). This will give rise to a trade-off in the convergence, i.e., the distance error does not converge to the origin but to a bounded set, the size of which depends on how large the deadzone parameter is chosen;

- Use the hysteresis principle in the quantization function design;

- Use the self-triggering principle, as in [Persis and Frasca, 2013].

The adoption of the above techniques to avoid chattering will be discussed in future research. 


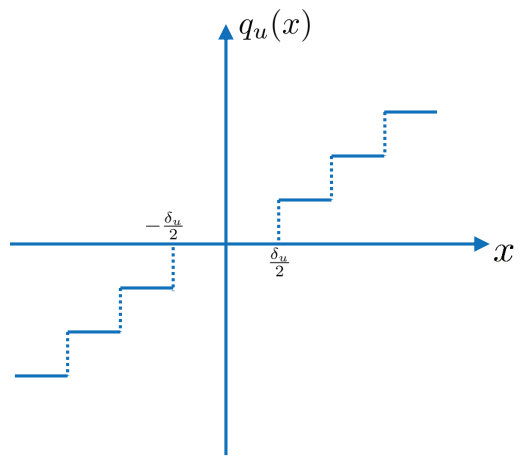

(a)

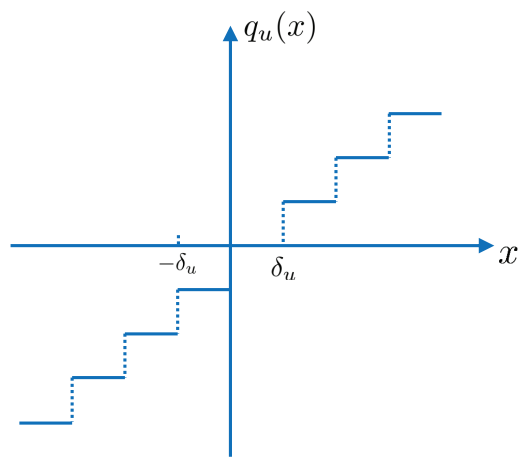

(b)

Figure 7.2: (a) Symmetric uniform quantizer function, defined in (7.2). (b) Asymmetric uniform quantizer function, defined in (7.23).

\subsection{Asymmetric uniform quantizer}

In [Liu et al., 2012], it has been shown that when an asymmetric uniform quantizer (defined below) is applied to double-integrator consensus dynamics some undesirable motions may occur. In this section we investigate whether there are undesired motions for rigid formation control in the presence of an asymmetric uniform quantizer.

We consider the following asymmetric uniform quantizer (the same as in [Liu et al., 2012]), defined by

$$
q_{u}^{*}(x)=\delta_{u}\left(\left\lfloor\frac{x}{\delta_{u}}\right\rfloor\right)
$$

where $\delta_{u}$ is a positive number and $\lfloor a\rfloor, a \in \mathbb{R}$ denotes the greatest integer that is less than or equal to $a$. For a comparison of the uniform quantizers defined in (7.2) and in (7.23), see Figure 7.2.

\section{7·5.1 Motivating example: two-agent formation case}

We first consider a two-agent formation case. Suppose two agents are controlled to achieve an inter-agent distance of $d_{12}$ with the quantization function (7.23). The system dynamics for agents 1 and 2 can be described, respectively, as

$$
\dot{p}_{1}=q_{u}^{*}\left(\left\|z_{1}\right\|-d_{12}\right) \hat{z}_{1}
$$

and

$$
\dot{p}_{2}=-q_{u}^{*}\left(\left\|z_{1}\right\|-d_{12}\right) \hat{z}_{1}
$$

where $z_{1}=p_{2}-p_{1}$, and $q_{u}^{*}(\cdot)$ denotes the asymmetric uniform quantizer in (7.23).

Lemma 30. Consider the two-agent formation control system (7.24) and (7.25) with the 
asymmetric quantization function (7.23).

- If the initial distance between agents 1 and 2 is greater than $d_{12}+\delta_{u}$, then the interagent distance $\|z\|$ will converge to $d_{12}+\delta_{u}$ and the final formation will be stationary;

- If the initial distance between agents 1 and 2 is smaller than the desired distance $d_{12}$, then the inter-agent distance $\|z\|$ will converge to the desired distance $d_{12}$ and the final formation will be stationary;

- If the initial distance between agents 1 and 2 is between $d_{12}$ and $d_{12}+\delta_{u}$, then both agents 1 and 2 are stationary and the inter-agent distance $\|z\|$ remains unchanged.

The proof is obvious and is omitted here as it can be inferred from previous proofs.

Remark 19. In the above example it can be seen that in the case of an asymmetric uniform quantizer, there exist no undesired motions, which is different to the result observed in [Liu et al., 2012] which showed unbounded velocities. Apart from the difference in system dynamics under discussions, the key difference that leads to the distinct behaviors is that when the asymmetric quantizer is applied to the consensus dynamics (which is to quantize a vector), there holds $\mathcal{F}\left[q_{u}^{*}\left(r_{i}-r_{j}\right)\right]+\mathcal{F}\left[q_{u}^{*}\left(r_{j}-r_{i}\right)\right]=-\delta_{u}$ when $r_{j}-r_{i} \neq k \delta_{u}$, where $r_{j}-r_{i}$ denotes the relative position vector (see Section 5 of [Liu et al., 2012]). Note that in the above formation controller, the quantization applies only to the distance error term (i.e. $\left.q_{u}^{*}\left(\left\|p_{2}-p_{1}\right\|-d_{12}\right)\right)$ which is a scalar, and the asymmetric property of the quantizer only affects the convergence of the distance term.

\subsubsection{General formation case}

We consider the general formation case with more than two agents, in which each agent employs asymmetric uniform quantizers in individual controllers.

Theorem 13. Suppose each individual agent takes the asymmetric uniform quantizer (7.23) in the quantized formation controller (7.7). Then the inter-agent distances converge to the set

$$
F_{\text {aym }}=\left\{e \mid e_{k} \in\left[0, \delta_{u}\right], k \in\{1, \ldots, m\}\right\} .
$$

The proof is omitted here as it can be directly inferred from previous proofs.

\subsection{Illustrative examples and simulations}

In this section we show several examples to illustrate the theoretical results obtained in previous sections. In the following illustrative examples we consider the stabilization control of a five-agent minimally rigid formation in the 3-D space. The underlying graph describes a double tetrahedron shape of nine edges, and the desired distances for all edges are set as $6 .{ }^{2}$ The initial positions are chosen such that the

\footnotetext{
${ }^{2}$ Note that the realization of a target formation with the given nine desired distances is not unique up to rotation and translation [Hendrickson, 1992].
} 

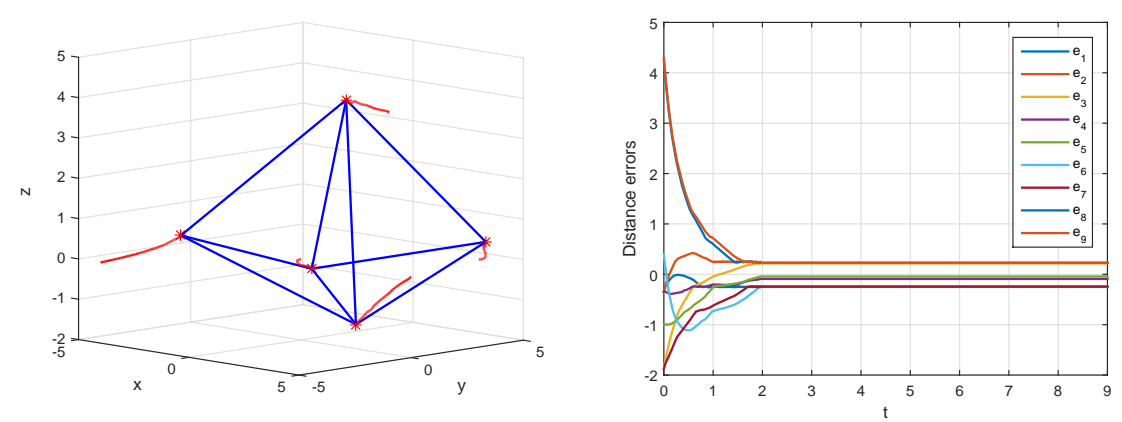

Figure 7.3: Stabilization control of a rigid formation: symmetric uniform quantization case. Left: the trajectories of five agents and the final formation shape. Right: Time evolutions of the distance errors.

initial formation is infinitesimally rigid and is close to a target formation shape. For all simulations, we set the quantization gain as $\delta_{u}=0.5$.

Agents trajectories, the final formation shape and the evolutions of nine distance errors under symmetric uniform quantization and under logarithmic quantization are shown in Figure 7.3 and Figure 7.4, respectively. It is obvious from these two figures that with symmetric uniform quantizer the formation errors converge to the bounded set $F_{\text {approx }}=\left\{e \mid e_{k} \in[-0.25,0.25], k \in\{1, \ldots, m\}\right\}$, and with the logarithmic quantizer the formation converges to the target shape, which are consistent with the theoretical results in Theorem 11.

The formation convergence behavior with binary distance measurements under the quantization strategy (7.17) is depicted in Figure 7.5. It can be seen from Figure 7.5 that with very coarsely quantized distance measurement via a simple signum function as in (7.17), the formation converges to the target shape within a finite time, but the price to be paid is the occurrence of chattering (as shown in the right part of Figure 7.5).

Finally, when the asymmetric uniform quantizer (7.23) is used in the formation control system (7.7), the formation converges to an approximate one with all distance errors converging to the bounded set $F_{\text {aym }}=\left\{e \mid e_{k} \in[0,0.5], k \in\{1, \ldots, m\}\right\}$, as shown in Figure 7.6. This supports the conclusion of Theorem 13.

\subsection{Concluding remarks}

In this chapter we consider the rigid formation control problem with quantized distance measurements. We have discussed in detail the quantization effect on the convergence of rigid formation shapes under two commonly-used quantizers. In the case of the symmetric uniform quantizer, all distances will converge locally to a bounded set, the size of which depends on the quantization error. In the case of the logarithmic quantizer, all distances converge locally to the desired values. We also consider a special quantizer with a signum function, which allows each agent to use very coarse distance measurements (i.e. binary information on whether it is close 

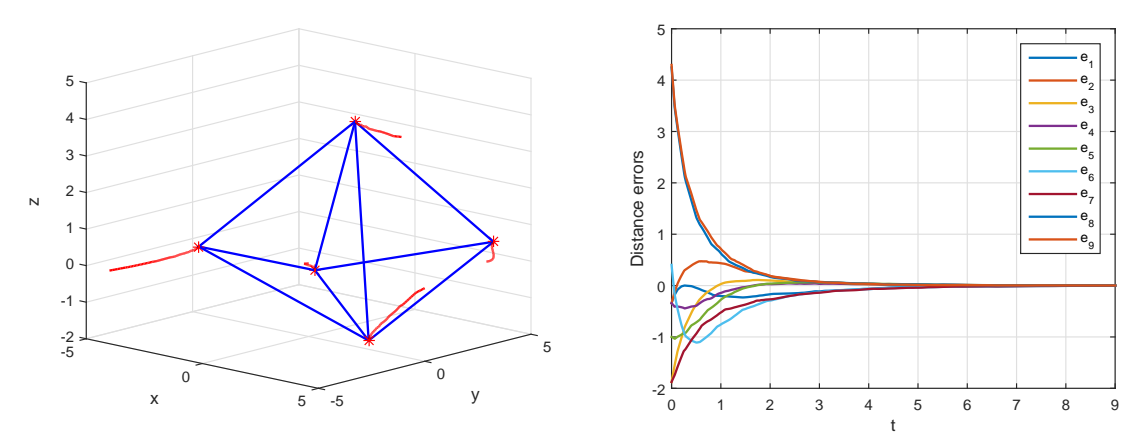

Figure 7.4: Stabilization control of a rigid formation: logarithmic quantization case. Left: the trajectories of five agents and the final formation shape. Right: Time evolutions of the distance errors.
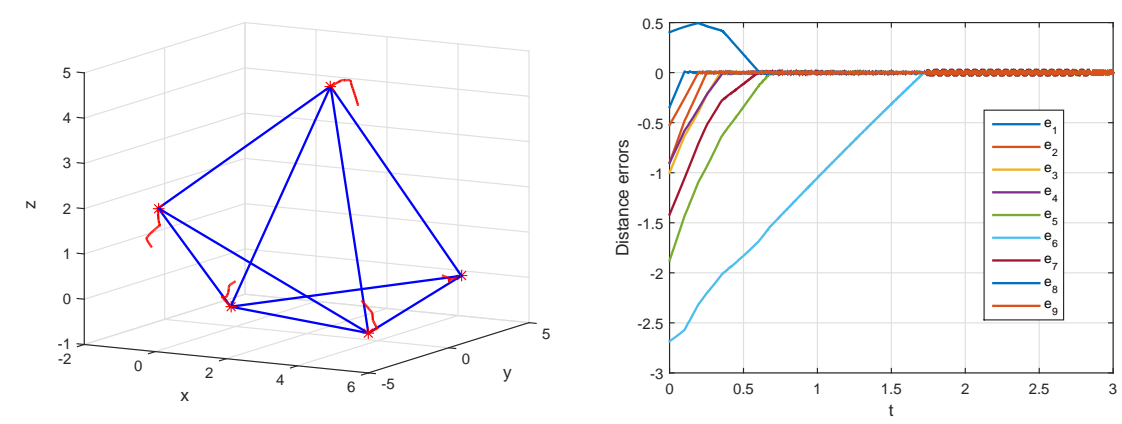

Figure 7.5: Stabilization control of a rigid formation: binary measurement case. Left: the trajectories of five agents and the final formation shape. Right: Time evolutions of the distance errors.
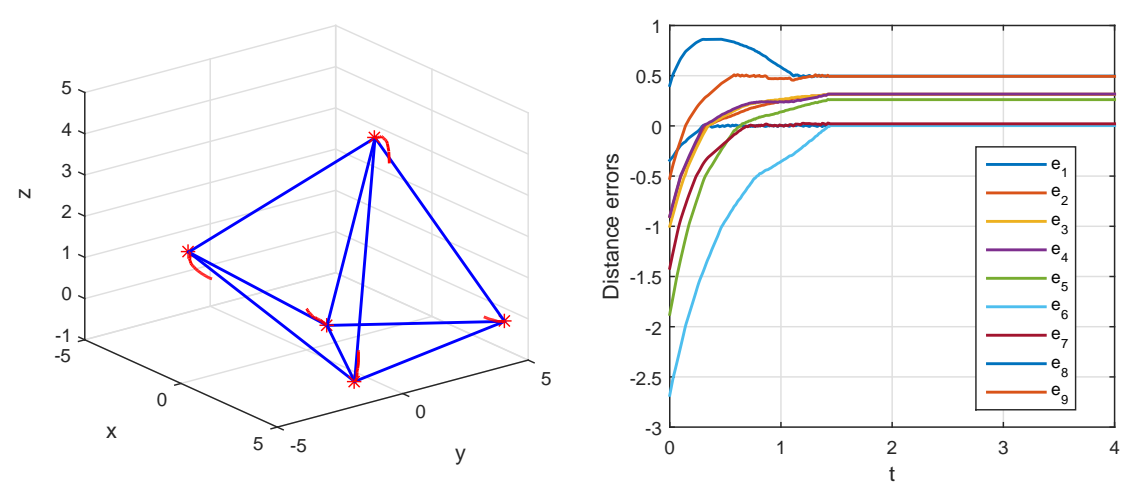

Figure 7.6: Stabilization control of a rigid formation: asymmetric uniform quantization case. Left: the trajectories of five agents and the final formation shape. Right: Time evolutions of the distance errors. 
or far away to neighboring agents with respect to the desired distances). We show in this case the formation shape can still be achieved within a finite time. We further discuss the case of an asymmetric quantizer applied in rigid formation control system, and analyze the convergence property of distance errors.

\subsection{Appendix: background and useful tools on nonsmooth analysis}

\section{Calculus for computing differential inclusion}

In the following we list some properties of calculus for computing Filippov's differential inclusion.

1. Assume that $f, g: \mathbb{R}^{m} \rightarrow \mathbb{R}^{n}$ are locally bounded; then $\mathcal{F}[f+g](x) \subset \mathcal{F}[f](x)+$ $\mathcal{F}[g](x)$.

2. Let $g: \mathbb{R}^{m} \rightarrow \mathbb{R}^{n}$ be $C^{1}$ (i.e. continuously differentiable function), $\operatorname{rank}(D g(x))=$ $n$ and $f: \mathbb{R}^{n} \rightarrow \mathbb{R}^{p}$ be locally bounded; then $\mathcal{F}[f \circ g](x)=\mathcal{F}[f](g(x))$.

3. Let $g: \mathbb{R}^{m} \rightarrow \mathbb{R}^{p \times n}$ be $C^{0}$ (i.e. continuous function) and $f: \mathbb{R}^{m} \rightarrow \mathbb{R}^{n}$ be locally bounded; then $\mathcal{F}[g f](x)=g(x) \mathcal{F}[f](x)$, where $g f(x):=g(x) f(x) \in \mathbb{R}^{p}$.

4. Assume that $f_{j}: \mathbb{R}^{m} \rightarrow \mathbb{R}^{n_{j}}, j \in\{1,2, \cdots, N\}$ are locally bounded; then $\mathcal{F}\left[\times_{j=1}^{N} f_{j}\right](x) \subset \times_{j=1}^{N} \mathcal{F}\left[f_{j}\right](x)$.

The proof for the above properties can be found in [Paden and Sastry, 1987].

\section{A collection of definitions: generalized derivative and gradient}

Definition 7. (see [Clarke, 1998]) Let $f: \mathbb{R}^{m} \rightarrow \mathbb{R}$. The right directional derivative $f^{\prime}(x ; v)$ of $f$ at $x$ in the direction of $v \in \mathbb{R}^{d}$ is defined as

$$
f^{\prime}(x ; v)=\lim _{h \rightarrow 0^{+}} \frac{f(x+h v)-f(x)}{h}
$$

Definition 8. (see [Clarke, 1998]) Let $f: \mathbb{R}^{m} \rightarrow \mathbb{R}$. The generalized derivative $f^{o}(x ; v)$ of $f$ at $x$ in the direction of $v \in \mathbb{R}^{d}$ is defined as

$$
\begin{aligned}
f^{o}(x ; v) & =\limsup _{\substack{y \rightarrow x \\
h \rightarrow 0^{+}}} \frac{f(y+h v)-f(y)}{h} \\
& =\lim _{\substack{\delta \rightarrow 0^{+} \\
\epsilon \rightarrow 0^{+}}} \sup _{\substack{y \in \mathcal{B}(x, \delta) \\
h \rightarrow[0, \epsilon)}} \frac{f(y+h v)-f(y)}{h}
\end{aligned}
$$

Definition 9. (see [Clarke, 1998]) A function $f$ is called regular at $x$ if $f^{o}(x ; v)=f^{\prime}(x ; v)$ for all $v \in \mathbb{R}^{d}$. 
Definition 10. (see [Clarke, 1998]) If $f: \mathbb{R}^{m} \rightarrow \mathbb{R}$ is locally Lipschitz, then its generalized gradient $\partial f: \mathbb{R}^{d} \rightarrow 2^{\mathbb{R}^{d}}$ is defined by

$$
\partial f(x)=\operatorname{co}\left\{\lim _{i \rightarrow \infty} \nabla f\left(x_{i}\right) \mid x_{i} \rightarrow x, x_{i} \notin S \cup \Omega_{f}\right\}
$$

where $\Omega_{f}$ denotes the set of points where $f$ fails to be differentiable and $S \subset \mathbb{R}^{d}$ is a set of measure zero.

We refer the readers to [Clarke, 1998] for more discussions on generalized derivative and function regularity in nonsmooth analysis.

\section{Lyapunov theory in nonsmooth analysis}

Definition 11. (see [Cortés, 2008]) Given a locally Lipschitz function $f: \mathbb{R}^{m} \rightarrow \mathbb{R}$, the set-valued Lie derivative of $f$ with respect to the Filippov set-valued map $\mathcal{F}[x]$ at $x$ is defined as

$$
\begin{aligned}
\tilde{\mathcal{L}}_{\mathcal{F}[X]} f(x)= & \{a \in \mathbb{R} \mid \exists v \in \mathcal{F}[X](x) \\
& \text { such that } \left.\zeta^{\top} v=a, \forall \zeta \in \partial f(x)\right\} .
\end{aligned}
$$

If $\mathcal{F}[X](x)$ takes convex and compact values, then for each $x \in \mathbb{R}^{d}, \tilde{\mathcal{L}}_{\mathcal{F}[X]} f(x)$ is a closed and bounded interval in $\mathbb{R}$, possibly empty. For the empty set, we adopt the convention $\max (\varnothing)=-\infty$. If $f$ is continuously differentiable at $x$, then

$$
\dot{\bar{V}}(x):=\tilde{\mathcal{L}}_{\mathcal{F}[X]} f(x)=\left\{(\nabla f)^{\top} \cdot v \mid v \in \mathcal{F}[X](x)\right\} .
$$

As discussed in [Cortés, 2008], the set-valued Lie derivative allows us to study how a function $f$ evolves along the solutions of a differential inclusion without having to explicitly obtain the solutions. The usefulness of the set-valued Lie derivative is highlighted by the following theorem.

Theorem 14. (see [Bacciotti and Ceragioli, 1999]) Let $x:\left[t_{0}, t_{1}\right] \rightarrow \mathbb{R}^{n}$ be a Filippov solution of $\dot{x}(t) \in \mathcal{F}[X] x(t)$. Let $V(x)$ be a locally Lipschitz and regular function. Then $(d / d t) V(x(t))=\nabla V^{\top} \dot{x}$ exists almost everywhere and

$$
(d / d t) V(x(t)) \in \dot{\bar{V}}(x)
$$

holds almost everywhere.

Theorem 15. (see [Bacciotti and Ceragioli, 1999]) Let $f: \mathbb{R}^{m} \rightarrow \mathbb{R}$ be a locally Lipschitz and regular function. Let $S$ be compact and strongly invariant for the differential inclusion $\dot{x}(t) \in \mathcal{F}[X] x(t)$, and assume that $\max \tilde{\mathcal{L}}_{\mathcal{F}[X]} f(x) \leq 0$ for each $x \in S$. Define $Z_{X, f}=$ $\left\{x \in \mathbb{R}^{d} \mid 0 \in \tilde{\mathcal{L}}_{\mathcal{F}[X]} f(x)\right\}$. Then all solutions $x:[0, \infty) \rightarrow \mathbb{R}^{d}$ of the differential inclusion converge to the largest weakly invariant set $M$ contained in

$$
S \cap \overline{Z_{X, f}}
$$


The word weakly, as opposed to the word strongly, is used in the above theorem to indicate that at least one solution (as opposed to all solutions) satisfies the invariant set property. The proof can be found in [Bacciotti and Ceragioli, 1999]. For more discussions on regular functions and weakly invariant sets, we refer the readers to [Cortés, 2008].

\section{Additional calculus rules}

The following theorem provides a convenient way to calculate the generalized derivative and to apply Lyapunov theory for nonsmooth systems.

Theorem 16. (see [Shevitz et al., 1994]) Let $x(\cdot)$ be a Filippov solution to $\dot{x}=f(x, t)$ on an interval containing $t$ and $V: \mathbb{R}^{n} \times \mathbb{R} \rightarrow \mathbb{R}$ be a Lipschitz and regular function. Then $V(x(t), t)$ is absolutely continuous, $(d / d t) V(x(t), t)$ exists almost everywhere and

$$
\frac{d}{d t} V(x(t), t) \in^{\text {a.e. }} \dot{\tilde{V}}(x(t), t)
$$

where

$$
\dot{\tilde{V}}(x(t), t):=\bigcap_{\xi \in \partial V(x(t), t)} \xi^{\top}\left(\begin{array}{c}
\mathcal{F}[f](x(t), t) \\
1
\end{array}\right)
$$

The proof can be found in [Shevitz et al., 1994]. Note there also holds $\dot{\bar{V}}(x) \subset$ $\dot{\tilde{V}}(x(t))$ (see e.g. [Bacciotti and Ceragioli, 1999]).

\section{Finite-time convergence}

The following theorem further characterizes finite time convergence under stronger conditions than the above Theorem 15.

Theorem 17. (see [Cortés, 2006]) Under the same assumptions of Theorem 15, further assume that there exists a neighborhood $U$ of $S \cap Z_{X, f}$ in $S$ such that max $\tilde{\mathcal{L}}_{\mathcal{F}[X]} f(x) \leq-\epsilon<0$ a.e. on $U\left(S \cap Z_{X, f}\right)$. Then any solution $x:[0, \infty) \rightarrow \mathbb{R}^{d}$ of the differential inclusion starting at $x_{0} \in S$ reaches $S \cap Z_{X, f}$ in finite time. Moreover, if $U=S$, then the convergence time is upper bounded by $\left(f\left(x_{0}\right)-\min _{x \in S} f(x)\right) / \epsilon$.

The proof can be found in [Cortés, 2006]. 
Part III

\section{Distributed Coordination Control: \\ General System Models}



Chapter 8

\section{Rigid formation control of double-integrator systems}

\section{Chapter summary}

In this chapter, we move our focus from formation control systems modelled by single integrators (e.g. those considered in previous chapters) to formation systems modelled by double integrators. Two kinds of double-integrator formation systems are considered, namely, formation stabilization systems and flocking control systems. Novel observations on the measurement requirement, the null space and eigenvalues of the system Jacobian matrix will be provided, which reveal important properties of system dynamics and the associated convergence results. We also establish some new links between single-integrator formation systems and double-integrator formation systems via a parameterized Hamiltonian system, which in addition provide novel stability criteria for different equilibria in double-integrator formation systems by using available results from single-integrator formation systems.

\subsection{Introduction}

\subsubsection{Background and related work}

In the literature, most results on rigid formation control are based on simple singleintegrator formation models; see e.g. [Dimarogonas and Johansson, 2008], [Krick et al., 2009], [Dörfler and Francis, 2010], [Helmke and Anderson, 2013], [Tian and Wang, 2013], [Anderson and Helmke, 2014], [Rozenheck et al., 2015] and the survey [Oh et al., 2015]. In this chapter, we will consider formation control systems modelled by double integrators, motivated by the fact that double-integrator systems serve as a somewhat more natural models to describe many real-life control systems.

Double-integrator models have been studied extensively for flocking control of multi-agent systems, partly originated by the pioneering works [Olfati-Saber, 2006] and [Tanner et al., 2007]. Another active research topic on double-integrator models which received particular interest in recent years is the linear consensus problem [Ren, 2008], [Cao et al., 2013]. However, for rigid formation control systems modelled 
by double integrators, the results appear rather sparsely in the literature, with some discussions in [Dimarogonas and Johansson, 2008], [Oh and Ahn, 2014a], [Sun et al., 2014b], [Ramazani et al., 2015; Cai and De Queiroz, 2014; Zhang et al., 2015]. A recent paper [Deghat et al., 2016] showed the possibility of combining a linear consensus algorithm and a nonlinear rigid shape controller to achieve a desired rigid flocking movement. Note that all these papers only focused on some local convergence analysis. Due to the nonlinear property of the formation controller for stabilizing rigid shapes, a full characterization of the convergence analysis is quite challenging. Actually, there are many open issues for rigid formation control systems when they are modelled by double integrators, which include equilibrium properties, convergence analysis, robustness issues, etc.

This chapter provides new results dealing with the system dynamics and convergence property of double-integrator rigid formation systems. We will consider two types of formation systems: formation stabilization systems and flocking control systems. The main contributions of this chapter are summarized as follows. First, we will characterize null spaces and zero eigenvalues of the Jacobian matrix for the vector function of double-integrator formation systems, which will reveal several system dynamical properties. Second, compared with the analysis and results in [Dimarogonas and Johansson, 2008], [Oh and Ahn, 2014a], [Sun et al., 2014b], [Ramazani et al., 2015; Cai and De Queiroz, 2014; Zhang et al., 2015] and [Deghat et al., 2016], we go well beyond the local convergence analysis of the correct equilibrium set (i.e., those equilibria corresponding to correct formation shapes). Instead, we aim to provide new characterizations for the convergence properties of any equilibrium set, including those that do not correspond to a desired equilibrium. Third, invariant properties and links between single-integrator formation systems and double-integrator formation systems will be established. This will be done by employing a parameterized Hamiltonian system, an idea which was also used for power network analysis [Chiang and $\mathrm{Wu}, 1988$ ], [Chiang and $\mathrm{Chu}, 1995]$, oscillator networks [Dörfler and Bullo, 2011], and was briefly mentioned in a recent paper [Oh and Ahn, 2014a] on formation control. We will show how available results on characterizing equilibrium properties in single-integrator rigid formation systems (e.g. [Krick et al., 2009; Dörfler and Francis, 2010; Park et al., 2014; Sun et al., 2015a]) can be readily extended to the stability analysis for double-integrator formation systems. In our opinion, this is the most revealing of any method for analyzing the stability of double-integrator systems, since it ties their properties so closely to those of the more easily understood single-integrator rigid formation systems.

We mention that in this chapter we do not focus on proposing novel controllers for stabilizing rigid formation shapes. Instead, we study double-integrator rigid formation systems with the popular formation stabilization controller shown in (2.10), while the main purposes of this chapter are to provide novel understanding and more insights on the system dynamics and convergence analysis which will help the implementation of such formation controllers in practice. 


\subsubsection{Chapter organization}

The remaining parts of this chapter are organized as follows. Section 8.2 briefly reviews some background and then introduces the relevant system equations. Section 8.3 presents results on local convergence and Jacobian matrix analysis using linearization technique. By exploring a parameterized Hamiltonian system, Section 8.4 establishes certain invariance principles, which help to characterize novel equilibrium properties on double-integrator formation systems by relating them to available results in single-integrator formation systems. Extensions of the results to flocking formation systems are discussed in Section 8.5. Some illustrative examples with simulation results are shown in Section 8.6. Finally, conclusions are provided in Section 8.7 which closes this chapter.

\subsection{Motion equations}

Let $d_{k_{i j}}$ denote the desired length of edge $k$ which links agents $i$ and $j$. We assume that the set of desired lengths is realizable in the given $d$-dimensional Euclidean space, i.e., there exists a formation in $\mathbb{R}^{d}$ whose inter-agent distances correspond to the desired values. ${ }^{1}$ In the following, the set of all formations $(\mathcal{G}, p)$ which satisfies the distance constraints in $\mathbb{R}^{d}$ is referred to as the set of target formations. ${ }^{2}$ We assume here that the target formation is infinitesimally rigid.

\subsubsection{Introduction of double-integrator formation control systems}

For the aim of comparisons and convenience of analysis, we recall the following formation control system modelled by a single integrator from Chapter 2:

$$
\dot{p}_{i}=-\sum_{j \in \mathcal{N}_{i}}\left(\left\|p_{i}-p_{j}\right\|^{2}-d_{k_{i j}}^{2}\right)\left(p_{i}-p_{j}\right), \quad i=1, \ldots, n
$$

which defines the steepest descent gradient flow of the distance potential function

$$
V(p)=\frac{1}{4} \sum_{(i, j) \in \mathcal{E}}\left(\left\|p_{i}-p_{j}\right\|^{2}-d_{k_{i j}}^{2}\right)^{2}
$$

In a compact form, we can rewrite (8.1) as

$$
\dot{p}(t)=-\nabla_{p} V=-R^{\top}(z) e(z)
$$

Two kinds of double-integrator rigid formation systems will be considered in this

\footnotetext{
${ }^{1}$ Suppose the target formation can be realized in an ambient space $\mathbb{R}^{d}$ (thus it can be realized in any ambient space with dimension $d^{\prime}>d$ ). Then there exists an ambient space $\mathbb{R}^{\tilde{d}}$ with a minimal dimension $\tilde{d}$, such that the target formation cannot be realized in $\mathbb{R}^{d^{\prime}}$ for any $d^{\prime}<\tilde{d}$. In later analysis of this chapter we will also occasionally use the concept minimal dimension when appropriate.

${ }^{2}$ Again, we remark that the realization of a target formation with the given desired distances may not be unique up to rotation and translation [Hendrickson, 1992].
} 
chapter. The first one is a model with velocity damping term, which aims to stabilize a desired rigid shape and to achieve a stationary formation (i.e. the final formation should come to a rest). The formation system can be described by the following form:

$$
\begin{aligned}
& \dot{p}_{i}=v_{i} \\
& \dot{v}_{i}=-k_{i} v_{i}-\sum_{j \in \mathcal{N}_{i}}\left(\left\|p_{i}-p_{j}\right\|^{2}-d_{k_{i j}}^{2}\right)\left(p_{i}-p_{j}\right)
\end{aligned}
$$

where $v_{i} \in \mathbb{R}^{d}$ is the velocity of agent $i$ and $k_{i}$ is a positive control gain which is freely selectable by the system designer. Define

$$
\psi(p, v):=\frac{1}{2} \sum_{i \in \mathcal{V}}\left\|v_{i}\right\|^{2}+V(p)
$$

Then in a compact form, we can rewrite the above double-integrator formation stabilization system as

$$
\begin{aligned}
\dot{p} & =\nabla_{v} \psi=v \\
\mathcal{H}_{\text {formation }}: \dot{v} & =-\mathcal{K} \nabla_{v} \psi-\nabla_{p} \psi \\
& =-\mathcal{K} v-R^{\top}(z) e(z)
\end{aligned}
$$

where $\mathcal{K}=K \otimes I_{d \times d}$ and $K$ is a diagonal gain matrix with the $i$-th diagonal entry being $k_{i}$.

The other model is for achieving a flocking behavior with both velocity consensus and shape stabilization. The overall system can be described by the following equations ${ }^{3}$

$$
\begin{aligned}
& \dot{p}_{i}=v_{i} \\
& \dot{v}_{i}=\sum_{j \in \mathcal{N}_{i}}\left(v_{j}-v_{i}\right)-\sum_{j \in \mathcal{N}_{i}}\left(\left\|p_{i}-p_{j}\right\|^{2}-d_{k_{i j}}^{2}\right)\left(p_{i}-p_{j}\right)
\end{aligned}
$$

In a compact form, we can rewrite the above double-integrator flocking control system as

$$
\mathcal{H}_{\text {flocking }}: \begin{aligned}
& \dot{p}=\nabla_{v} \psi=v \\
& \dot{v}=-\mathcal{L} v-R^{\top}(z) e(z)
\end{aligned}
$$

where $\mathcal{L}=L \otimes I_{d \times d}$ and $L$ is the Laplacian matrix for the underlying undirected and

\footnotetext{
${ }^{3}$ Note that there are two types of velocity consensus algorithms depending on different underlying graphs: one is based on undirected underlying graph and the other is based on directed graph (for achieving a leader-following control). Furthermore, the underlying graph for achieving velocity consensus can be different to the one of shape stabilization (see relevant discussions in [Deghat et al., 2016; Qin and $\mathrm{Yu}, 2013]$ ). In this chapter we focus on the first one (with undirected underlying graph for the velocity consensus) and assume the same underlying graph for both shape stabilization and velocity consensus.
} 
connected graph.

It is noted that different distance potential functions, other than the one in (8.2), can be chosen for stabilizing rigid formation shapes, which will give rise to different types of formation controllers. For the case of general potential functions, we have provided a unified approach for conducting convergence analysis in Chapter 4 (or see relevant discussions in [Sun et al., 2016a]). In this chapter, we will focus on the potential function (8.2) to model double-integrator formation systems, while more general potential functions can also be used by following the similar analysis to that in Chapter 4.

\subsubsection{Independence of global coordinate frame}

We state the following lemma on the coordinate system requirement for implementing the formation control law (8.6) and the flocking control law (8.8).

Lemma 31. Suppose each agent can access its own velocity (which can be measured in its own coordinate basis). Then the implementation of the double-integrator formation control system (8.6) does not require all agents' coordinate systems to be aligned. Furthermore, suppose each agent can measure the relative velocity to its neighbors in addition to its own velocity (which can be measured in its own coordinate basis). Then the implementation of the double-integrator flocking system (8.8) does not require all agents' coordinate systems to be aligned.

Proof. The proof for the first statement in the lemma can be handled in the same way as the proof of the second statement, so we will focus on the proof of the second statement.

Suppose agent $i^{\prime}$ s position and velocity in the global coordinate system $\sum_{g}$ are measured as $p_{i}^{g}$ and $v_{i}^{g}$, while $p_{i}^{i}, p_{j}^{i}, v_{i}^{i}$, and $v_{j}^{i}$ denote agent $i^{\prime}$ 's and its neighboring agent $j$ 's position and velocity, respectively, measured by agent $i$ 's local coordinate system. The controller for agent $i$ is implemented in its local coordinate as

$$
\ddot{p}_{i}^{i}=u_{i}^{i}=\sum_{j \in \mathcal{N}_{i}}\left(v_{j}^{i}-v_{i}^{i}\right)-\sum_{j \in \mathcal{N}_{i}}\left(\left\|p_{i}^{i}-p_{j}^{i}\right\|^{2}-d_{k_{i j}}^{2}\right)\left(p_{i}^{i}-p_{j}^{i}\right)
$$

Clearly, there exist a rotation matrix $\mathcal{Q}_{i} \in S O(d)$ and a translation vector $\vartheta_{i} \in \mathbb{R}^{d}$, such that $p_{j}^{i}=\mathcal{Q}_{i} p_{j}^{g}+\vartheta_{i}$ and $v_{j}^{i}-v_{i}^{i}=\mathcal{Q}_{i}\left(v_{j}^{g}-v_{i}^{g}\right)$. We rewrite the controller (8.9) for agent $i$ in the global coordinate system $\sum_{g}$ as follows

$$
\begin{aligned}
\ddot{p}_{i}^{g} & =\mathcal{Q}_{i}^{-1} u_{i}^{i}=\mathcal{Q}_{i}^{-1}\left(\sum_{j \in \mathcal{N}_{i}}\left(v_{j}^{i}-v_{i}^{i}\right)-\sum_{j \in \mathcal{N}_{i}}\left(\left\|p_{i}^{i}-p_{j}^{i}\right\|^{2}-d_{k_{i j}}^{2}\right)\left(p_{i}^{i}-p_{j}^{i}\right)\right) \\
& =\sum_{j \in \mathcal{N}_{i}}\left(v_{j}^{g}-v_{i}^{g}\right)-\sum_{j \in \mathcal{N}_{i}}\left(\left\|p_{i}^{g}-p_{j}^{g}\right\|^{2}-d_{k_{i j}}^{2}\right) \mathcal{Q}_{i}^{-1} \mathcal{Q}_{i}\left(p_{i}^{g}-p_{j}^{g}\right) \\
& =\sum_{j \in \mathcal{N}_{i}}\left(v_{j}^{g}-v_{i}^{g}\right)-\sum_{j \in \mathcal{N}_{i}}\left(\left\|p_{i}^{g}-p_{j}^{g}\right\|^{2}-d_{k_{i j}}^{2}\right)\left(p_{i}^{g}-p_{j}^{g}\right)
\end{aligned}
$$


which has the same form as (8.9). Since $\mathcal{Q}_{i}$ and $\vartheta_{i}$ are chosen arbitrarily, the above equation indicates that the designed controllers for all agents are independent of the global coordinate basis. The proof for the first statement can be handled by modifying $v_{j}^{i}-v_{i}^{i}$ as $v_{i}^{i}$ in the above equation, and a similar argument then follows.

In the remaining analysis in later sections, in order to avoid complicated notation, we will drop the superscript in the controller term (8.9) or (8.10) and still use the expression of (8.4) or (8.7) for the convenience of analysis.

Remark 20. The above controller property, termed the translational and rotational invariance property (or the SE(d) invariance property [Vasile et al., 2015]), has been well studied for single-integrator rigid formation control systems (see e.g. [Krick et al., 2009]). To the best of our knowledge, the corresponding property for double-integrator rigid formation systems has been largely ignored in the previous literature. Lemma 31 highlights the advantage of rigid formation controllers, in contrast to the displacement-based formation control strategy (see the survey [Oh et al., 2015]).

Remark 21. In practical formation control one needs to distinguish different ways of obtaining the required relative information for each individual agent (e.g., either by measurement solely at one agent or measurement additionally at neighboring agents with communication). In the statement of Lemma 31, we assume the relative velocity $v_{j}-v_{i}$ is measured using agent $i$ 's local coordinate system which is usually the case for implementing a formation control system in practice. If alternatively, the neighboring agents' velocities were transmitted to agent $i$, then adjacent agents would need to align their local coordinate systems or have the knowledge of the orientation of a common coordinate system so that the communicated velocity information can be correctly interpreted.

\subsection{System dynamics}

\subsubsection{Equilibrium sets for single- and double-integrator formation sys- tems}

We first describe the equilibrium set of each formation system. Suppose the distance potential (8.2) and the target formation are the same for the single-integrator system (8.3) and double-integrator systems (8.6) (used in the composite potential (8.5)) and

(8.8). Denote the set of all equilibria of the single-integrator system (8.3) as

$$
\mathcal{M}_{S}:=\left\{p^{*}: \nabla_{p} V\left(p^{*}\right)=\mathbf{0}\right\}
$$

First note that the distance potential $V$ defined in (8.2) for shape stabilization only depends on distances between agents and thus is invariant with respect to translation and rotation. Such symmetries imply that any formation system that involves the gradient of the distance potential $V$ in (8.2) does not have isolated equilibrium points. Instead, it possesses continuum equilibria which form orbits under the $S E(d)$ group 
action. To fix ideas, let us define the $S E(d)$ group action to a configuration $P=$ $\left[p_{1}, p_{2}, \cdots, p_{n}\right]$ of $n$ agents' positions with each $p_{i} \in \mathbb{R}^{d}$ :

$$
\mathcal{O}_{P}:=S E(d) \cdot P=\left[\mathcal{Q} p_{1}+\vartheta, \mathcal{Q} p_{2}+\vartheta, \cdots, \mathcal{Q} p_{n}+\vartheta\right]
$$

where $\mathcal{Q} \in S O(d)$ and $\vartheta \in \mathbb{R}^{d}$ are components of $S E(d)$. From this it is obvious to see that if $p^{*}$ is an equilibrium point of (8.3), then any point in the orbit generated by the group action $\mathcal{O}_{P^{*}}$ in (8.12) is also an equilibrium point. Similarly, according to the notion used in [Helmke and Anderson, 2013] and [Chen et al., 2015], we will call the orbit associated with an equilibrium point $p^{*}$ generated by the group action $\mathcal{O}_{P^{*}}$ an equilibrium orbit. As a consequence, the Hessian for the gradient-based rigid formation system (8.3) is always singular, with parts of the null spaces induced by the shape invariance under rotation and translation. If the Hessian at an equilibrium point $p^{*}$ of (8.3) is nonsingular in the normal direction to $\mathcal{O}_{P^{*}}$, then we will say it is normal hyperbolic.

Similarly, for the corresponding double-integrator formation system (8.6), the set of all equilibria can be described as

$$
\mathcal{M}_{D}:=\left\{\left(p^{*}, v^{*}\right): \nabla_{p} V\left(p^{*}\right)=\mathbf{0}, v^{*}=\mathbf{0} \in \mathbb{R}^{d n}\right\}
$$

Finally, for the double-integrator flocking system (8.8), we define the set

$$
\mathcal{M}_{F}:=\left\{\left(p^{*}, v^{*}\right): \nabla_{p} V\left(p^{*}\right)=\mathbf{0}, \mathcal{L} v^{*}=\mathbf{0}\right\}=\left\{\left(p^{*}, v^{*}\right): \nabla_{p} V\left(p^{*}\right)=\mathbf{0}, v^{*}=\mathbf{1}_{n} \otimes v_{c}\right\}
$$

where $v_{c}$ is the average velocity. In the later analysis, we will call the set (8.14) defined above the set of equilibrium orbits for the double-integrator flocking system, according to the fact that all agents will finally show a steady equilibrium motion which is a flocking motion. The above discussions on continuum equilibria and equilibrium orbits under $S E(d)$ group action for the single-integrator system (8.3) also apply to the property of the equilibrium set defined above for the double-integrator formation stabilization system (8.6) and flocking system (8.8).

By the definition in (8.14), $v_{c}$ is the velocity of the formation centroid. The existence of a constant $v_{c}$, readily computable from the initial conditions, is shown by the following lemma.

Lemma 32. For the double-integrator flocking system (8.8), the velocity of the formation centroid $v_{c}$ remains fixed throughout the motion.

Proof. Denote by $p_{c} \in \mathbb{R}^{d}$ the center of the mass of the formation, i.e., $p_{c}=\frac{1}{n} \sum_{i=1}^{n} p_{i}=$ $\frac{1}{n}\left(\mathbf{1}_{n} \otimes I_{d \times d}\right)^{\top} p$ and thus

$$
v_{c}=\dot{p}_{c}=\frac{1}{n}\left(\mathbf{1}_{n} \otimes I_{d \times d}\right)^{\top} \dot{p}
$$


From (8.8), one can show

$$
\begin{aligned}
\dot{v}_{c} & =\frac{1}{n}\left(\mathbf{1}_{n} \otimes I_{d \times d}\right)^{\top} \ddot{p} \\
& =\frac{1}{n}\left(\mathbf{1}_{n} \otimes I_{d \times d}\right)^{\top}\left(-\mathcal{L} v-R^{\top} e\right) \\
& =-\frac{1}{n}\left(\mathbf{1}_{n} \otimes I_{d \times d}\right)^{\top} \mathcal{L} v-\frac{1}{n}\left(Z^{\top}\left(H \otimes I_{d \times d}\right)\left(\mathbf{1}_{n} \otimes I_{d \times d}\right)\right)^{\top} e
\end{aligned}
$$

Note that $\left(\mathbf{1}_{n} \otimes I_{d \times d}\right)^{\top} \mathcal{L} v=\mathbf{0}$. Also because null $(H)=\operatorname{span}\left\{\mathbf{1}_{n}\right\}$ there holds

$$
\left(Z^{\top}\left(H \otimes I_{d \times d}\right)\left(\mathbf{1}_{n} \otimes I_{d \times d}\right)\right)^{\top} e=\mathbf{0} .
$$

Thus $\dot{v}_{c}=\mathbf{0}$, which indicates that the velocity of the formation centroid remains constant.

Based on Lemma 32, a commonly-used approach for stability analysis of the flocking control system is to perform a linear system transformation by separating the motion of the formation centroid (see e.g., [Deghat et al., 2016]). We will also discuss this point in Section 8.5.

In the following, we introduce the notion of set stability [Hahn and Baartz, 1967, Chapter V] which will be used later for the stability analysis of equilibrium sets or equilibrium orbits. Define the distance between a point $p$ and a set $S$ as

$$
\operatorname{dist}(p, S)=\inf _{y \in S}\|p-y\|
$$

This point-to-set distance allows us to define the stability for a set, which can be seen as a straightforward extension of the well known definition of that for an equilibrium point. We omit the detailed definitions here, and refer the readers to [Hahn and Baartz, 1967, Chapter V] for definitions and explanations on concepts such as set stability, asymptotic set stability and exponential set stability. The slight difference between the set stability and a closely related concept termed orbital stability (see [Wiggins, 2003, Chapter 1.2] for detailed discussions) was discussed in [Hahn and Baartz, 1967, Page 171], and there will be no confusion caused by this slight difference in the context of formation control (since an equilibrium orbit under $S E(d)$ group action is also defined as an equilibrium set). Therefore, when no confusion is expected, we will use these two terms orbital stability and set stability interchangeably.

In general, rigid formation control systems exhibit multiple equilibrium orbits (see discussions in [Anderson and Helmke, 2014], [Sun et al., 2015a]). We call the set of equilibria at which the correct shape is attained the correct equilibrium set (or correct equilibrium orbit), and we denote it by $\mathcal{M}_{S}^{c}:=\left\{p^{*}: e\left(p^{*}\right)=\mathbf{0}\right\}$ for the singleintegrator system (8.3), $\mathcal{M}_{D}^{c}=\left\{\left(p^{*}, \mathbf{0}\right): e\left(p^{*}\right)=\mathbf{0}\right\}$ for the double-integrator formation system (8.6), and $\mathcal{M}_{F}^{c}=\left\{\left(p^{*}, v^{*}\right): e\left(p^{*}\right)=\mathbf{0}, v^{*}=\mathbf{1}_{n} \otimes v_{c}\right\}$ for the doubleintegrator flocking system (8.8) ${ }^{4}$. Note that a correct equilibrium set is characterized

\footnotetext{
${ }^{4}$ In the set $\mathcal{M}_{F}^{c}$ all agents under the control law of (8.8) reach a steady flocking behavior in which
} 
by the condition $e\left(p^{*}\right)=\mathbf{0}$, i.e., the set of desired distances at such an equilibrium is achieved. Correspondingly, one can also define incorrect equilibrium set by following the property that at such an equilibrium the set of desired distances is not achieved (i.e. $e\left(p^{*}\right) \neq \mathbf{0}$ ).

Remark 22. We also note that the set of correct equilibria (up to translation, rotation and reflection) may not form a unique equilibrium orbit. This is because the realization of a target formation with given distances (up to translation, rotation and reflection) may not be unique due to e.g., flex or flip ambiguity [Hendrickson, 1992]. In other words, two correct equilibria may not correspond to congruent formations, since for minimally rigid formations with more than three agents, there always exist noncongruent equilibria pairs obtainable by either a flex or flip ambiguity [Anderson et al., 2008b].

\subsubsection{General results on convergence analysis}

The following convergence results for double-integrator formation systems (8.6) and (8.8) are well known, and are based on a standard Lyapunov argument and LaSalle's Invariance Principle.

Lemma 33. Each trajectory of the double-integrator formation system (8.6) converges to an invariant set in $\mathcal{M}_{D}$. Also, each trajectory of the double-integrator flocking system (8.8) converges to an invariant set in $\mathcal{M}_{F}$.

The proof can be shown by following similar steps from e.g. [Deghat et al., 2016], [Sun et al., 2016b] and is omitted here.

The following result characterizes the local convergence of the correct equilibrium set.

Corollary 5. There exists a neighborhood of $\mathcal{M}_{D}^{c}$ such that the trajectory of (8.6) converges locally to an equilibrium point in $\mathcal{M}_{D}^{c}$. Also, there exists a neighborhood of $\mathcal{M}_{F}^{c}$ such that the trajectory of (8.8) converges locally to $\mathcal{M}_{F}^{c}$.

We remark that the first statement in Corollary 5 indeed shows a stronger convergence result, i.e., the convergence to a point in the equilibrium set (rather than the convergence to the set).

The local convergence to the correct equilibrium set for double-integrator formation systems as shown in Corollary 5 has been proved in several previous papers based on different assumptions on graph topologies and formation characterizations. For example, in [Dimarogonas and Johansson, 2008], the local convergence for double-integrator formation stabilization system (8.6) was proved by assuming that the underlying topology is a tree (and in this case the local convergence to a correct equilibrium set can be extended to almost global convergence). In [Sun et al., 2014b], the local convergence for the double-integrator flocking system (8.8) was proved by

they also achieve a velocity consensus. The term equilibrium to describe the set $\mathcal{M}_{F}^{c}$ for (8.8) may seem unusual since at a point of $\mathcal{M}_{F}^{c}$ the right-hand side of (8.8) is not zero. However, we keep this terminology as it will be clear if one considers a reduced-order flocking system by factoring out the translational motion; see discussions in Section 8.4.3. 
assuming that the target formation is minimally and infinitesimally rigid, via Lyapunov arguments and LaSalle's Invariance Principle. A more recent paper [Deghat et al., 2016] presented a comprehensive study for the double-integrator formation flocking system (8.8), by employing the linearization approach and the Center Manifold Theorem [Carr, 1981]. Note that [Deghat et al., 2016] only assumed that the target formation is infinitesimally rigid, which is a more relaxed assumption than that in [Sun et al., 2014b]. Also note that [Deghat et al., 2016] mostly focused on the stability analysis for the formation flocking system (8.8), while the analysis and proof can be directly extended to the case of formation stabilization system (8.6). Hence, we omit the proof for Corollary 5 here and refer the readers to the above references.

Note that the above results only concern the local convergence property of the correct equilibrium set. In Section 8.4.2 we will prove the instability for a special set of incorrect equilibria called degenerate equilibria after we establish a link between single-integrator rigid formation systems and double-integrator rigid formation systems.

\subsubsection{Jacobian matrix analysis}

In this subsection we will show new results on system dynamics at a general equilibrium by analyzing the Jacobian matrix via the linearization technique. From this subsection we will focus on the double-integrator formation control system (8.6) for the convenience of analysis, but note that most results can be extended to the corresponding flocking model (8.8) with slight modifications. In Section 8.5, we will show how to extend the results from the formation control system (8.6) to the flocking control system (8.8).

Denote the Jacobian matrix of the right-hand vector function of (8.3) at an equilibrium point $p^{*}$ as

$$
\begin{aligned}
J_{p^{*}} & =\left.\frac{\partial\left(-R^{\top}(p) e(p)\right)}{\partial p}\right|_{p=p^{*}} \\
& =\left.\left(-\frac{\partial R^{\top}(p)}{\partial p} e(p)-R^{\top}(p) \frac{\partial e(p)}{\partial p}\right)\right|_{p=p^{*}}
\end{aligned}
$$

Note that because (8.3) describes a gradient descent flow of $V(p), J_{p^{*}}$ is actually the Hessian matrix of $-V(p)$ and thus is symmetric. Define a diagonal matrix $E=$ $\operatorname{diag}\left(e_{1}, e_{2}, \cdots, e_{m}\right) \in \mathbb{R}^{m \times m}$. Then an explicit expression of $J_{p^{*}}$ can be obtained as (see e.g. [Anderson and Helmke, 2014], [Sun et al., 2015a])

$$
\begin{aligned}
J_{p^{*}} & =-2 R\left(p^{*}\right)^{\top} R\left(p^{*}\right)-\left(H^{\top} E\left(p^{*}\right) H\right) \otimes I_{d \times d} \\
& =\bar{H}^{\top}\left(-2 Z\left(p^{*}\right) Z\left(p^{*}\right)^{\top}-E\left(p^{*}\right) \otimes I_{d \times d}\right) \bar{H}
\end{aligned}
$$

Note that, as can be seen from the above formula (8.18), the null space of $J_{p^{*}}$ is always non-empty. For example, in the 2-D case, the dimension of $\operatorname{null}\left(J_{p^{*}}\right)$ is at least three, with two dimensions reflecting the translation invariance and one dimension 
reflecting the rotation invariance of a rigid shape. In the general case, suppose the inter-agent distances defining a target formation are realized in a given ambient space with a minimal dimension $d$. Then at a correct equilibrium at which $E\left(p^{*}\right)$ is a zero matrix, there holds $J_{p^{*}}=-2 R\left(p^{*}\right)^{\top} R\left(p^{*}\right)$ and the dimension of the null space of $J_{p^{*}}$ is $d(d+1) / 2$ according to Theorem 1 in Chapter 2 .

The Jacobian matrix of the double-integrator formation system (8.6) at an equilibrium point $\left(p^{*}, \mathbf{0}\right)$ can be calculated as

$$
J\left(p^{*}, \mathbf{0}\right)=\left[\begin{array}{cc}
\mathbf{0}_{d n \times d n} & I_{d n \times d n} \\
J_{p^{*}} & -\mathcal{K}
\end{array}\right]
$$

where $J_{p^{*}}$ is the Jacobian matrix of (8.3) defined in (8.17), and $\mathcal{K}$ is the diagonal gain matrix defined in (8.6).

The following result characterizes the null space of $J\left(p^{*}, \mathbf{0}\right)$.

Theorem 18. Suppose the null space of $J_{p^{*}}$ is spanned by a set of linearly independent vectors $v_{j}$ and denote the null space by null $\left(J_{p^{*}}\right)=\operatorname{span}\left\{v_{1}, \cdots, v_{j}, \cdots, v_{l}\right\}$. Define the corresponding vector $v_{j}=\left[v_{j}^{\top}, \mathbf{0}^{\top}\right]^{\top}$. Then there holds null $\left(J\left(p^{*}, \mathbf{0}\right)\right)=\operatorname{span}\left\{v_{1}, \cdots, v_{j}, \cdots, v_{l}\right\}$.

Proof. Suppose $v_{j}=\left[v_{j p}^{\top}, v_{j v}^{\top}\right]^{\top}$ is a vector in the null space of $J\left(p^{*}, \mathbf{0}\right)$, that is

$$
\left[\begin{array}{cc}
\mathbf{0}_{d n \times d n} & I_{d n \times d n} \\
J_{p^{*}} & -\mathcal{K}
\end{array}\right]\left[\begin{array}{c}
v_{j p} \\
v_{j v}
\end{array}\right]=0
$$

which can be simplified as

$$
\begin{aligned}
v_{j v} & =\mathbf{0} \\
J_{p^{*}} v_{j p}-\mathcal{K} v_{j v} & =\mathbf{0}
\end{aligned}
$$

The above equation implies that $v_{j p}$ is the corresponding vector in the null space of $J_{p^{*}}$. Thus, there holds $v_{j}:=v_{j p}$. Also note that each vector $v_{j}$ in the null space of $J_{p^{*}}$ gives rise to one vector $v_{j}$ in the null space of $J\left(p^{*}, \mathbf{0}\right)$. Since each $v_{j}$ is linearly independent, it follows that each $v_{j}$ is also linearly independent which serves as a valid basis for the null space of $J\left(p^{*}, \mathbf{0}\right)$. Hence the statement is proved.

The above analysis further leads to the following lemma, which can be easily proved by observing the size of the matrices and the dimensions of the null spaces.

Lemma 34. Suppose at an equilibrium $p^{*}$, the rank of the Jacobian matrix $J_{p^{*}}$ for the singleintegrator system (8.3) is $k$. Then at the corresponding equilibrium $\left(p^{*}, \mathbf{0}\right)$ the rank of the Jacobian matrix $J\left(p^{*}, \mathbf{0}\right)$ for the double-integrator system (8.6) is $d n+k$.

We next show a result on an eigenvalue property of $J\left(p^{*}, \mathbf{0}\right)$.

Lemma 35. With the positive definite gain matrix $\mathcal{K}$ as defined previously, the eigenvalues of $J\left(p^{*}, \mathbf{0}\right)$ cannot be purely imaginary. 
Proof. Let $\lambda$ be an eigenvalue of $J\left(p^{*}, \mathbf{0}\right)$ with the corresponding eigenvector denoted by $v_{\lambda}=\left[v_{\lambda, p}^{\top}, v_{\lambda, v}^{\top}\right]^{\top}$,

$$
\left[\begin{array}{cc}
\mathbf{0}_{d n \times d n} & I_{d n \times d n} \\
J_{p^{*}} & -\mathcal{K}
\end{array}\right]\left[\begin{array}{l}
v_{\lambda, p} \\
v_{\lambda, v}
\end{array}\right]=\lambda\left[\begin{array}{l}
v_{\lambda, p} \\
v_{\lambda, v}
\end{array}\right]
$$

The above equation can be further rewritten as

$$
\begin{aligned}
v_{\lambda, v} & =\lambda v_{\lambda, p} \\
J_{p^{*}} v_{\lambda, p}-\mathcal{K} v_{\lambda, v} & =\lambda v_{\lambda, v}
\end{aligned}
$$

Multiplying $v_{\lambda, p}^{*}$ (i.e. the complex conjugate transpose of $v_{\lambda, p}$ ) to both sides of the above equation, one can get

$$
\lambda^{2} v_{\lambda, p}^{*} v_{\lambda, p}+\lambda v_{\lambda, p}^{*} \mathcal{K} v_{\lambda, p}-v_{\lambda, p}^{*} J_{p^{*}} v_{\lambda, p}=0
$$

Note that $v_{\lambda, p}$ cannot be a zero vector (otherwise $v_{\lambda}$ will be a zero vector which then contradicts the fact that $v_{\lambda}$ is an eigenvector). Also note that the coefficient $v_{\lambda, p}^{*} \mathcal{K} v_{\lambda, p}$ is positive because $\mathcal{K}$ is positive definite. Hence there cannot exist purely imaginary roots to the above quadratic equation (8.24), which implies that the eigenvalues of $J\left(p^{*}, \mathbf{0}\right)$ cannot be purely imaginary at any equilibrium.

Remark 23. Note that when $\mathcal{K}=0$ (the damping term is zero) then the above doubleintegrator system (8.6) describes a Hamiltonian system. We note one consequence of the above Lemma 35: The non-existence of purely imaginary eigenvalues of the Jacobian $J\left(p^{*}, \mathbf{0}\right)$ implies that Hopf bifurcation in the double-integrator formation system (8.6) cannot occur.

In the next section, we will interpret these various properties (including the null space properties) of the Hessian and of the Jacobian in the light of convergence of rigid formation systems.

\subsection{Perspectives from a parameterized double-integrator for- mation system}

One of the main aims of this chapter is to characterize the convergence properties of the double-integrator system (8.6) (and (8.8)) in terms of those of the reduced-order single-integrator formation system (8.3). In this section, we will consider a family of parameterized formation systems which provides a bridge between the singleintegrator gradient formation system (8.3) and the double-integrator formation system (8.6) (and further the flocking control system (8.8)). Two extreme values of the relevant parameter effectively correspond to single- and double-integrator formation systems. Intermediate values of the parameter do not correspond to formations, but are relevant to the definition of a sort of homotopy between the two types of formation. This parameterization-based idea is inspired by [Chiang and $\mathrm{Wu}, 1988$ ], [Chiang 
and Chu, 1995] and [Dörfler and Bullo, 2011], in which similar approaches were employed for the stability and convergence analysis of Hamiltonian-like power systems ([Chiang and $\mathrm{Wu}, 1988]$, [Chiang and Chu, 1995]) and oscillator networks ([Dörfler and Bullo, 2011]). A recent paper [Oh and Ahn, 2014a] has briefly discussed this idea in the rigid formation shape control with double-integrator systems. The use of this idea in the context of formation control with shape stabilization and flocking is however novel, which will reveal several important insights on the stability analysis of different types of equilibrium points for double-integrator formation systems.

Consider the double-integrator system with a parameter $\lambda \in[0,1]$ in the following form

$$
\mathcal{H}_{\lambda}:\left[\begin{array}{c}
\dot{p} \\
\dot{v}
\end{array}\right]=\underbrace{\left[\begin{array}{cc}
-\lambda I & (1-\lambda) I \\
-(1-\lambda) I & -\mathcal{K}
\end{array}\right]}_{=: S_{\lambda}}\left[\begin{array}{c}
\nabla_{p} \psi \\
\nabla_{v} \psi
\end{array}\right]
$$

In the case that $\lambda=0$, the above system (8.25) reduces to the double-integrator formation stabilization system shown in (8.6). In the case that $\lambda=1$, the above system (8.25) then reduces to the following uncoupled gradient system:

$$
\begin{aligned}
& \dot{p}=-\nabla_{p} \psi \\
& \dot{v}=-\mathcal{K} \nabla_{v} \psi
\end{aligned}
$$

\subsubsection{Invariance principles}

In the following, we will prove an invariance result which represents a substantial extension of that in [Oh and Ahn, 2014a]. This result helps to relate a gradient system (8.26) and a double-integrator formation stabilization system (8.6) via the parameterized Hamiltonian system (8.25).

Theorem 19. For the one-parameter family of dynamical systems $\mathcal{H}_{\lambda}$ in (8.25), the following statements hold:

- Invariance of equilibrium sets: For all $\lambda \in[0,1]$, the equilibrium set of $\mathcal{H}_{\lambda}$ is given by the set of critical points of the potential function $\psi$ (i.e. the critical points of (8.26)) and is independent of $\lambda$;

- Invariance of the inertia: For any equilibrium of $\mathcal{H}_{\lambda}$ for all $\lambda \in[0,1]$, the inertia of the Jacobian of the vector function of $\mathcal{H}_{\lambda}$ is equal to that of the Hessian of $\psi$ and is independent of $\lambda$.

Proof. Note that $\mathcal{K}$ is positive definite and is invertible. By the Schur Determinant Formula [Zhang, 2006, Theorem 1.1] it follows from the definition of $S_{\lambda}$ in (8.25) that $\operatorname{det}\left(S_{\lambda}\right)=\operatorname{det}(-\mathcal{K}) \operatorname{det}\left(-\lambda I+(1-\lambda)^{2}(-\mathcal{K})^{-1}\right)$, which is non-zero for all $\lambda \in$ $[0,1]$. Hence, the matrix $S_{\lambda}$ is nonsingular for all $\lambda \in[0,1]$, which implies that the equilibrium set of $\mathcal{H}_{\lambda}$ for which the right-hand side of (8.25) is zero is identical with the equilibrium set for which the right-hand side of (8.26) is zero, irrespective of the 
value of $\lambda \in[0,1]$. That is, the equilibrium set of $\mathcal{H}_{\lambda}$ is given by the critical points of $\psi: \mathcal{M}_{\mathcal{H}_{\lambda}}=\left\{\left(p^{*}, v^{*}\right): \nabla_{p} \psi\left(p^{*}\right)=\mathbf{0}, \nabla_{v} \psi\left(v^{*}\right)=\mathbf{0}\right\}$ which is independent of the value of $\lambda$. Thus the first statement is proved.

The proof of the second statement is inspired by [Dörfler and Bullo, 2011]. Denote the Hessian of $\psi$ as $\nabla_{p, v}^{2} \psi$. The Jacobian of the vector function on the right-hand side of (8.25) can be written as $J_{\mathcal{H}_{\lambda}}=S_{\lambda} \nabla_{p, v}^{2} \psi$. Denote $A:=-J_{\mathcal{H}_{\lambda}^{\prime}}^{\top} P:=\nabla_{p, v}^{2} \psi$. By noting that $P$ is symmetric, one has

$$
\begin{aligned}
Q: & =\left(A P+P A^{\top}\right)=P\left(-S_{\lambda}-S_{\lambda}^{\top}\right) P \\
& =P\left[\begin{array}{cc}
2 \lambda I_{d n \times d n} & \mathbf{0}_{d n \times d n} \\
\mathbf{0}_{d n \times d n} & 2 \mathcal{K}
\end{array}\right] P
\end{aligned}
$$

It is obvious that $Q \succeq 0$ for $\lambda \geq 0$, and for $\lambda \neq 0$ we have $\operatorname{null}(Q)=\operatorname{null}(P)$. Hence, by [Carlson and Schneider, 1963, Theorem 5] one can conclude that the non-zero inertia (i.e. the number of $\left\{v_{+}, v_{-}\right\}$) of $A$ is the same as the non-zero inertia of $P$. Also note that $-J_{\mathcal{H}_{\lambda}}$ and $-J_{\mathcal{H}_{\lambda}}^{\top}$ have the same set of eigenvalues. These facts further imply that the non-zero inertia of $-J_{\mathcal{H}_{\lambda}}$ is determined by $\nabla_{p, v}^{2} \psi$ and is independent of $\lambda \in(0,1]$. We then consider the case of $\lambda=0$. Note that the eigenspace corresponding to the eigenvalues with zero real parts of $-J_{\mathcal{H}_{\lambda}}$ equals the eigenspace of zero eigenvalues of $\nabla_{p, v}^{2} \psi$ for all $\lambda \in[0,1]$, which implies that the non-zero inertia of $J_{\mathcal{H}_{\lambda}}$ cannot change when $\lambda=0$. In summary, the inertia of $-J_{\mathcal{H}_{\lambda}}$ equals the inertia of $\nabla_{p, v}^{2} \psi$ for all $\lambda \in[0,1]$, and thus the stability property of an equilibrium for (8.25) is determined by the inertia of the Hessian $\nabla_{p, v}^{2} \psi$ at that equilibrium and is independent of $\lambda$.

Remark 24. The above Theorem 19 can be seen as a generalization of [Oh and Ahn, 2014a, Theorem 4.1] (which again is an extension of [Dörfler and Bullo, 2011, Theorem 5.1]). This theorem was presented and used in [Oh and Ahn, 2014a] to show the local asymptotic convergence of a formation stabilization system with double integrators which focused on two special cases: $\lambda=1$ and $\lambda=0$. However, no proof was shown in [Oh and Ahn, 2014a]. We note that a proof for the above result is non-trivial, and we also extend the invariance results to the more general case for all $\lambda \in[0,1]$. Furthermore, a by-product of this Theorem 19 shows the equivalence between the eigenspace of the eigenvalues with zero real parts of $J\left(p^{*}, \mathbf{0}\right)$ and the null space of $S_{\lambda} \nabla_{p, v}^{2} \psi$, which generalizes the result in Theorem 18. In the following analysis in Section 8.4.3, we will show that Theorem 19 offers us additional insights to show the local exponential convergence.

\subsubsection{Relating double-integrator formation systems to single-integrator for- mation systems}

We now show more results to characterize different equilibrium sets of the doubleintegrator formation systems, by relating them to the available results in singleintegrator formation systems (e.g. the results discussed in Chapter 3). The following results are direct consequences of Theorem 19. 
Corollary 6. Suppose at an equilibrium $p^{*}$ in the equilibrium set $\mathcal{M}_{S}$, the Jacobian matrix $J_{p^{*}}$ for the single-integrator system (8.3) has $k$ (resp. $j$ ) positive (resp. negative) eigenvalues. Then at the corresponding equilibrium $\left(p^{*}, \mathbf{0}\right)$ in the equilibrium set $\mathcal{M}_{D}$ for the doubleintegrator system (8.6), the Jacobian matrix $J\left(p^{*}, \mathbf{0}\right)$ has $k(r e s p . d n+j)$ eigenvalues with positive (resp. negative) real parts.

As shown in Theorem 18, the Jacobian matrix $J\left(p^{*}, \mathbf{0}\right)$ for $(8.6)$ is always singular at every equilibrium point. Also, the Jacobian matrix $J_{p^{*}}$ (which is the Hessian of the negative potential $-V$ ) is always singular, with parts of the null space induced by the rotation and translation invariance of rigid formation shapes.

By assuming that the Hessian is normal hyperbolic (i.e. there is no extra zero eigenvalue for the Hessian other than those induced by shape invariance), Part II of Theorem 19 allows us to employ the inertia information of the Jacobian and of the Hessian to determine the local stability of an equilibrium set.

Corollary 7. (Property of equilibria in single- and double-integrator formation systems) Suppose the Hessian of the distance potential is normal hyperbolic at a particular equilibrium orbit, and for the single-integrator formation system (8.3), that particular equilibrium set is stable (resp. unstable). Then for the double-integrator formation system (8.6), the corresponding equilibrium set is stable (resp. unstable).

Theorem 19 and Corollary 7 imply that available results on stability analysis in single-integrator systems (e.g. [Krick et al., 2009], [Dörfler and Francis, 2010; Park et al., 2014; Sun et al., 2015a] and Chapter 3) can be applied here to determine whether an equilibrium set $\mathcal{M}_{D}\left(p^{*}, 0\right)$ is stable or not for the double-integrator formation stabilization system under question. In the following we will show an example.

Since the formation control system is a nonlinear system and it exhibits multiple equilibrium orbits, it is challenging to give a complete picture of the convergence property for different equilibrium sets. The following theorem borrows an existing result for single-integrator formation systems from Chapter 3 and confirms the instability for a particular set of incorrect equilibria.

In the following analysis, we assume that the target formation is realizable by a nondegenerate configuration in $\mathbb{R}^{d} .{ }^{5}$ Given an equilibrium $p^{*} \in \mathbb{R}^{n d}$ for the singleintegrator formation system (8.3), we construct a matrix $Z^{*}=\left[z_{1}^{*}, z_{2}^{*}, \cdots, z_{m}^{*}\right] \in \mathbb{R}^{d \times m}$ with the $i$-th column being the $i$-th relative position vector $z_{i}^{*}$ (see Chapter 2 for the definition and construction of relative position vectors $z$ ). We call an equilibrium point $p^{*}$ with $\operatorname{rank}\left(Z^{*}\right)<d$ an incorrect degenerate equilibrium for the single-integrator formation system (8.3), according to the fact that the affine space that embeds $p^{*}$ has dimension less than $d$ and at such a degenerate equilibrium the distance potential function $V(p)$ is not zero (i.e. the target shape is not achieved at $p^{*}$ ). For more properties such as existence of such equilibrium points, see Chapter 3. Accordingly, one

\footnotetext{
${ }^{5}$ For example, a target triangle formation shape with three given distances satisfying the triangle inequality can be realized in $\mathbb{R}^{2}$ but not in $\mathbb{R}^{1}$. In this case, any collinear equilibrium that only spans a $d=1$-dimensional affine space is a degenerate equilibrium.
} 
can define the degenerate equilibrium set for the double-integrator formation system (8.6) as

$$
\tilde{\mathcal{M}}_{D}:=\left\{\left(p^{*}, v^{*}\right): \nabla_{p} V\left(p^{*}\right)=\mathbf{0}, \operatorname{rank}\left(Z^{*}\right)<d, v^{*}=\mathbf{0} \in \mathbb{R}^{d n}\right\}
$$

Examples of degenerate equilibria include collinear equilibria (i.e. the equilibrium positions that span a one-dimensional affine space) for formations when agents live in $\mathbb{R}^{2}$, or coplanar equilibria (i.e. the equilibrium positions that span a two-dimensional affine space) for formations when agents live in $\mathbb{R}^{3}$. In the previous literature, properties of degenerate equilibria have been studied for single-integrator formation systems with some special formation shapes. For example, [Dörfler and Francis, 2010] and [Cao et al., 2011] studied in detail the collinear equilibria for a triangular formation target shape, while [Park et al., 2013] studied the properties of collinear equilibria and coplanar equilibria for a regular tetrahedron target shape. Note under the assumption that the target formation is realizable by a nondegenerate configuration in $\mathbb{R}^{d}$, the set of degenerate equilibria is a subset of the set of incorrect equilibria at which the formation has an incorrect shape.

According to the above invariance principle, one can obtain the following stability result.

Theorem 20. (Instability of degenerate equilibria) Suppose the target formation is realizable by a nondegenerate configuration in $\mathbb{R}^{d}$. A degenerate equilibrium set $\tilde{\mathcal{M}}_{D}$ for the double-integrator formation stabilization system (8.6) is unstable.

Proof. In Chapter 3, it has been proved that any degenerate equilibrium $p^{*}$ is unstable for the single-integrator formation system (8.3). As a consequence of Theorem 19, the degenerate equilibrium set $\tilde{\mathcal{M}}_{D}$ characterized by the rank condition $\operatorname{rank}\left(Z^{*}\right)<d$ is unstable for the corresponding double-integrator formation stabilization system (8.6).

Remark 25. Note that in Theorem 20 we do not need to assume that the Hessian of $-V$ (and the associated Jacobian $J\left(p^{*}, \mathbf{0}\right)$ ) are normal hyperbolic. This is because the existence of at least one negative eigenvalue ensures the existence of unstable manifold, which can be uniquely determined and is tangent to the unstable subspaces corresponding to the negative eigenvalue (c.f. the Nonhyperbolic Hartman-Grobman Theorem [Meiss, 2007, Page 189, Theorem 5.9]). As proved in [Sun et al., 2015a], unstable degenerate equilibria for singleintegrator formation systems are actually either local maximum or saddle points, and for saddle points there exist initial positions (the set of which has measure zero) forming an affine space of lower dimensions such that the single-integrator formation system cannot escape from that lower dimensional space and will converge to one of such degenerate equilibria. We will verify in simulations that, for double-integrator formation systems, even if agents start with initial positions that span an affine space with a dimension lower than the minimal dimension, the formation system can still converge to a correct formation shape instead of a degenerate equilibrium with an incorrect shape.

Remark 26. In many published papers on rigid formation control (see e.g. [Krick et al., 2009; Dörfler and Francis, 2010; Deghat et al., 2016; Sun et al., 2015a; Oh et al., 2015]), 
it is a standard assumption that the target formation is infinitesimally rigid, and during the convergence process the instantaneous formation shape at an arbitrary instant of time is assumed also to be infinitesimally rigid (i.e., the rigidity matrix always attains its maximum rank at any time during the convergence process). This cannot be achieved for arbitrary initial conditions, and for this reason most papers only consider local convergence, that is, the initial formation is chosen to be infinitesimally rigid and close to the target formation. Under this assumption, the infinitesimal rigidity of the instantaneous formation shape during the convergence can be guaranteed due to the gradient property of the dynamical system (which ensures that the norm of the distance error vector is non-increasing) and the continuous dependence of the rigidity matrix on agents' positions. In Section 8.4 .3 we will also use this assumption to ensure local exponential convergence. The rank-preserving property for singleintegrator rigid formation control systems, established in Chapter 3 and [Sun et al., 2015a], is however a different concept, which in most cases is not related to the rank of the rigidity matrix $R$. This can be seen from the definition of degenerate equilibria in (8.28) with the rank condition of the matrix $P$. The rank of $P$ is the dimension of the ambient affine space containing all agents, and is not the same to the rank of $R$ (or the infinitesimal rigidity as shown in Theorem 1). We have tried to apply the rank-preserving theory (see [Helmke and Moore, 1994, Chapter 5]) to determine whether the global preservation of the infinitesimal rigidity holds during the convergence under the gradient flow (8.3), but cannot obtain the result. This is because one cannot transform the matrix differential system involving $R$ into the standard form of a rank-preserving matrix differential equation shown in [Helmke and Moore, 1994, Lemma 1.22, Chapter 5]. There are some results on the global preservation of infinitesimal rigidity during the convergence for special formations (e.g. triangular formation [Dörfler and Francis, 2010], or tetrahedral formation [Park et al., 2014]), since in these special cases the rank of $P$ can be determined from the rank of $R$.

The following result further clarifies the topological conjugacy ${ }^{6}$ of the two trajectories from (8.6) and (8.26), which indicates that they can be continuously deformed to match each other while preserving the parameterization of time.

Theorem 21. (Topological conjugacy) Consider the double-integrator formation system (8.6) and the uncoupled single-integrator formation gradient system (8.26) modelled by the same undirected and rigid underlying graph and the same target formation. Then locally near a correct equilibrium set, their trajectories are topologically conjugate.

Proof. The proof is inspired by [Dörfler and Bullo, 2011, Theorem 5.3]. As discussed above, any correct equilibrium in the set of correct equilibria $\left(p^{*}, \mathbf{0}\right)$ for $(8.6)$ is not isolated but forms an equilibrium manifold with dimension $d(d+1) / 2$, which is induced by translational and rotational invariance. However, by factoring out the translational and rotational invariance, the correct equilibrium corresponds to an isolated equilibrium in a quotient space. Such a quotient space for the linearized system of (8.6) can be identified with the orthogonal complement of the null space induced by

\footnotetext{
${ }^{6}$ Two flows $\varphi_{t}: A \rightarrow A$ and $\psi_{t}: B \rightarrow B$ are conjugate if there exists a homeomorphism $h: A \rightarrow B$ such that for each $x \in A$ and $t \in \mathbb{R}$, there holds $h\left(\varphi_{t}(x)\right)=\psi_{t}(h(x))$. For more discussions on topological conjugacy, the readers are referred to [Meiss, 2007, Chapter 4.7].
} 
shape invariance, and the Jacobian matrix $J\left(p^{*}, \mathbf{0}\right)$ is normal hyperbolic (i.e. hyperbolic in that quotient space). According to the Hartman-Grobman Theorem [Meiss, 2007, Theorem 4.13], the trajectories of the vector fields (8.6) and (8.26) are locally topologically conjugate to the flow generated by their respective linearized vector fields near the correct equilibrium. Also as proved in Theorem 19, the linearized Jacobian matrix for each flow (8.6) and (8.26) has the same hyperbolic inertia (besides the same common center eigenspace of the same dimension induced by shape invariance). According to [Meiss, 2007, Theorem 4.12], this indicates that the corresponding linearized dynamics are topologically conjugate. In summary, the trajectories generated by the vector fields (8.6) and (8.26) are locally topologically conjugate near the correct equilibrium set.

We note that such a topological conjugacy property has been observed in several other practical systems modelled by double integrators, including power systems [Chiang and Chu, 1995] and oscillator synchronization [Dörfler and Bullo, 2011]. Here we show that it also holds for rigid formation control systems.

\subsubsection{Exponential convergence of double-integrator formation systems}

The following analysis in particular shows the convergence to a point in a correct equilibrium set and its convergence rate in double-integrator formation control systems under discussion. With a slight abuse of terminology, we will call the matrix $J_{p^{*}}$ normal negative definite if its null space only consists of the subspace induced by shape invariance and all non-zero eigenvalues are positive.

Lemma 36. (Local exponential convergence) The trajectory of (8.6) converges locally exponentially fast to an equilibrium point $\left(p^{*}, \mathbf{0}\right)$ in an equilibrium set, if and only if $J_{p^{*}}$ is normal negative definite at $p^{*}$ in the vector space orthogonal to the null space induced by shape invariance.

The proof for Lemma 36 is omitted but can be easily inferred from Theorem 19 as well as Corollary 6 and Corollary 7. By the assumption that the target formation is infinitesimally rigid, the above Lemma 36 indicates that the correct equilibrium is exponentially stable. The following gives a more detailed explanation on this exponential convergence statement.

Let us take 2-D formation systems as an example to interpret the above Lemma 36 and to determine the exponential convergence of double-integrator formation systems. From the characterization of the null space of the Jacobian matrix, the inertia of the Jacobian matrix at a point in that particular equilibrium orbit is independent of the choice of that point. If at an equilibrium point $p^{*}$ in a particular equilibrium set, the Jacobian $J_{p^{*}}$ of a 2-D single-integrator system (8.3) has inertia as $\left\{v_{+}, v_{-}, v_{0}\right\}=\{0,2 n-3,3\}$, then such equilibrium set is locally exponentially stable for (8.3). Actually, by invoking the Center Manifold Theorem [Carr, 1981] and by assuming that the target formation is infinitesimally rigid, it has been proven in [Krick et al., 2009] that the position of each agent in the gradient system $\dot{p}=-\nabla_{p} \psi$ in (8.26) 
converges locally exponentially fast to an equilibrium point in the correct equilibrium set, with the equilibrium point dependent on the initial condition. ${ }^{7}$

By combining this result with the statement in Lemma 36, one can show that if at an equilibrium point $\left(p^{*}, \mathbf{0}\right)$ in a particular equilibrium set $\mathcal{M}_{D}\left(p^{*}, \mathbf{0}\right)$, the Jacobian $J\left(p^{*}, \mathbf{0}\right)$ of a 2-D double-integrator system (8.6) has inertia as $\left\{v_{+}, v_{-}, v_{0}\right\}=$ $\{0,4 n-3,3\}$, then such an equilibrium set is locally exponentially stable and the trajectory of (8.6) converges locally exponentially fast to an equilibrium point in this equilibrium set. In particular, if the target formation is infinitesimally rigid, then the double-integrator formation system (8.6) converges locally exponentially fast to an equilibrium point in the correct equilibrium set with a desired formation shape.

Furthermore, according to the definition of the distance error vector $e$ in (2.11), we can conclude from the above discussion that inter-agent distances also converge locally exponentially fast to the desired values. It is worth mentioning that there exist other ways for proving local convergence for double-integrator formation systems (see e.g. [Oh and Ahn, 2014a] by using Łojasiewicz's inequality for a gradient flow, [Jiang et al., 2017] with the usage of the Malkin theorem, and [Dimarogonas and Johansson, 2008], [Sun et al., 2014b], [Zhang et al., 2015] by invoking Barbalat's Lemma and Lyapunov argument), all without however showing how fast the convergence is. Note that the exponential convergence delivered by our arguments is a crucial property for studying robustness issues in rigid formation control systems [Mou et al., 2016]. We mention that the key idea of Theorem 19 and Lemma 36 in this section has been employed in a recent paper [Garcia de Marina et al., 2016b] to study a double-integrator formation stabilization system with constant distance mismatches. In our future research, we will show that the exponential convergence established in this chapter is a key factor in explaining the robustness property of double-integrator rigid formation system and flocking system in the presence of more general perturbations.

\subsection{Extensions to double-integrator flocking systems}

At the beginning of Section 8.3 we have established explicit forms and some properties of equilibrium points for double-integrator flocking systems (8.8). In this section we will provide brief discussions to show that the obtained results in previous sections on double-integrator formation stabilization systems (8.6) can be extended to formation flocking systems (8.8). Note that in the formation flocking system, the motion of the whole formation can be broken into a combination of two motions [Deghat et al., 2016], i.e., that of the centroid, and that of the formation relative to the centroid. The latter motion is like that already analyzed in the previous sections. The former motion (that of the centroid) is simple to analyze. We now show how this works.

\footnotetext{
${ }^{7}$ Another way for proving the exponential convergence is to focus on the relative position dynamics or distance error dynamics; see e.g. [Dörfler and Francis, 2010; Sun et al., 2016a] and the exponential stability analysis in Chapter 4.
} 
Recalling the notations in Section 8.3.1, we have used $p_{c}$ to denote the formation centroid, and $v_{c}$ to denote the average velocity of all the agents, i.e., $v_{c}(t)=\dot{p}_{c}=$ $\frac{1}{n} \sum_{i=1}^{n} v_{i}(t)$. Observe that $v_{\mathcal{c}}(t)$ is time-invariant as shown in Lemma 32. We then define the relative position vector to the formation centroid as $\bar{p}_{i}=p_{i}-p_{c}$, and the relative velocity vector $\bar{v}_{i}=\dot{\bar{p}}_{i}=\dot{p}_{i}-\dot{p}_{c}$. Note that there holds $\bar{p}_{i}-\bar{p}_{j}=p_{i}-p_{j}$ and $\bar{v}_{i}-\bar{v}_{j}=v_{i}-v_{j}$. Also note there holds $\dot{\bar{v}}_{i}=\ddot{\bar{p}}_{i}$ since $v_{c}$ is constant. Hence, one can further transform (8.8) into the following equivalent system by factoring out the formation centroid dynamics:

$$
\begin{aligned}
& \dot{\bar{p}}_{i}=\bar{v}_{i} \\
& \dot{\bar{v}}_{i}=\sum_{j \in \mathcal{N}_{i}}\left(\bar{v}_{j}-\bar{v}_{i}\right)+\sum_{j \in \mathcal{N}_{i}} e_{k_{i j}}\left(\bar{p}_{j}-\bar{p}_{i}\right)
\end{aligned}
$$

By introducing the composite relative position vector $\bar{p}=\left[\bar{p}_{1}, \bar{p}_{2}, \cdots, \bar{p}_{n}\right]^{\top}$ and the velocity disagreement vector as $\bar{v}=\left[\bar{v}_{1}, \bar{v}_{2}, \cdots, \bar{v}_{n}\right]^{\top}$, one can rewrite the above system (8.29) in a compact form

$$
\begin{aligned}
& \dot{\bar{p}}=\bar{v} \\
& \dot{\bar{v}}=-\mathcal{L} \bar{v}-R^{\top}(\bar{p}) e(\bar{p})
\end{aligned}
$$

Since $\operatorname{null}(L)=\operatorname{span}\left\{\mathbf{1}_{n}\right\}$, there holds $\operatorname{null}(\mathcal{L})=\operatorname{span}\left\{\mathbf{1}_{n} \otimes I_{d \times d}\right\}$. Thus $\mathcal{L}$ is positive definite by restricting it to the vector space of $\operatorname{span}\left(\mathbf{1}_{n} \otimes I_{d \times d}\right)^{\perp}$ (where the superscript ${ }^{\perp}$ denotes orthogonal complement) in which the velocity disagreement vector $\bar{v}$ lives.

${ }^{8}$ From this viewpoint, $\mathcal{L}$ serves the same role as the positive definite gain matrix $\mathcal{K}$ in (8.6). Note that the equilibrium set $\mathcal{M}_{F}$ of (8.8) can be re-stated in the new coordinates as $\overline{\mathcal{M}}_{F,(8.30)}=\left\{\left(\bar{p}^{*}, \bar{v}^{*}\right): \nabla_{\bar{p}} V\left(\bar{p}^{*}\right)=\mathbf{0}, \bar{v}^{*}=\mathbf{0}\right\}$ for the transformed system (8.30). Thus, the transformed system (8.30) has the same structure as the doubleintegrator formation system (8.6), and all previous results on the equilibrium properties and parameterized system analysis for the double-integrator formation system (8.6) can be readily extended to (8.30).

\subsection{Illustrative examples on convergence}

In this section we consider several simulation examples on rigid formation control in the 3-D space. The target formation shape is supposed to be a double tetrahedron formation with 9 edges, with the desired distances for each edge being 6 . The initial positions and initial velocities for each agent are chosen randomly, but we also ensure that the initial formation shape is close to the target one. The formation stabilization result is shown in Figure 8.1, which illustrates the trajectories of each agent, together with the initial shape and final shape, and the trajectories of each distance error. The formation flocking result is shown in Figure 8.2, which illustrates the trajectories of

\footnotetext{
${ }^{8}$ Another way to perform the system transformation is to define the velocity disagreement vector with respect to the formation centroid velocity. Such a system transformation also gives rise to a reducedorder double-integrator formation system, as discussed in [Deghat et al., 2016].
} 

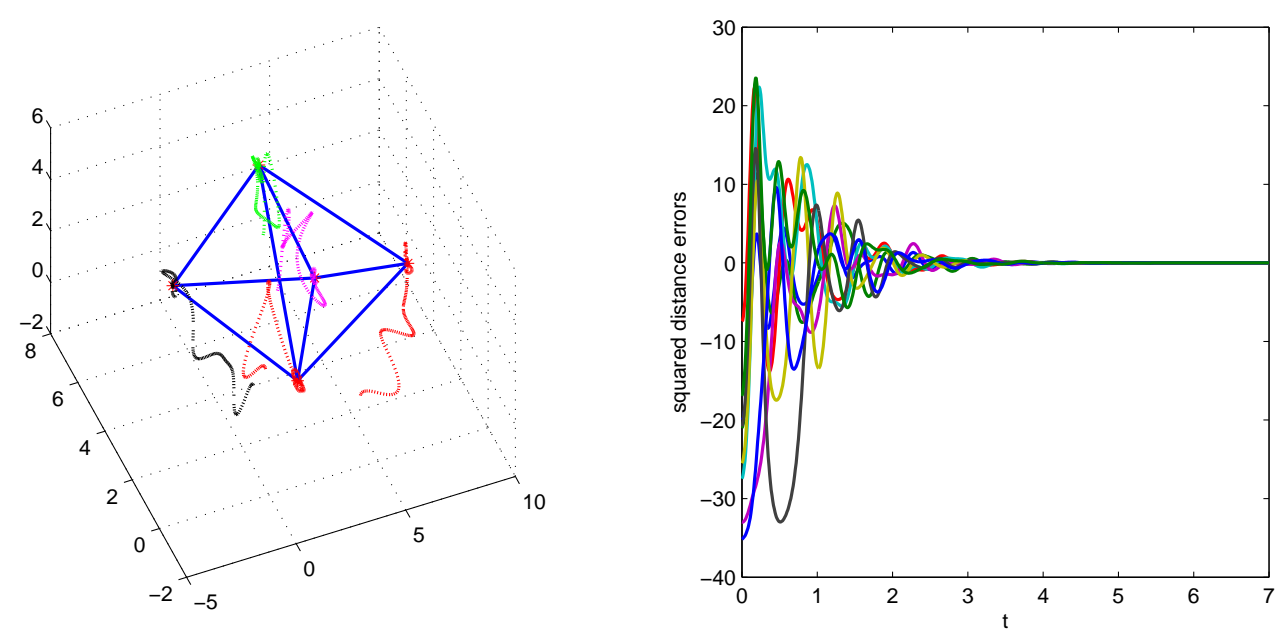

Figure 8.1: Simulation on shape stabilization control of a double tetrahedron formation in the 3-D space with double-integrator systems.

each agent, the flocking behavior, and the trajectories of each distance error. It can also be seen from simulations that the convergence of each distance error vector in both cases is exponentially fast.

Note that the above examples only show local convergence to the target formation shape. The following simulation shows a comparison of convergence results between single-integrator formation models and double-integrator formation models. We assume the same target formation shape as described above, and choose the initial positions for all agents as $p_{1}(0)=[4,4,0]^{\top}, p_{2}(0)=[0,0,0]^{\top}, p_{3}(0)=[4,0,0]^{\top}$, $p_{4}(0)=[5,0,0]^{\top}$ and $p_{5}(0)=[-4,-10,0]^{\top}$. The simulation result with singleintegrator model is depicted in Figure 8.3, which shows the convergence to an incorrect formation shape in a degenerate 2-D plane when agents' initial positions are chosen in that plane (which has measure zero in the whole space $\mathbb{R}^{3}$ ). The converged equilibrium in this case is $p_{1}^{*}=[3.2992,3.8112,0]^{\top}, p_{2}^{*}=[-1.5078,0.2206,0]^{\top}, p_{3}^{*}=$ $[4.0052,-2.1471,0]^{\top}, p_{4}^{*}=[4.0052,-2.1471,0]^{\top}$ and $p_{5}^{*}=[-0.8018,-5.7376,0]^{\top}$, which span the same plane that contains the initial positions. The convergence to an incorrect equilibrium (more specifically, a degenerate incorrect equilibrium) is due to the rank-preserving property of single-integrator rigid formation systems as discussed in [Sun et al., 2015a].

We then use the same initial positions (and random initial velocities) to perform the simulation with double-integrator models, and observe the convergence to the correct formation shape as shown in Figure 8.4. Actually, as proved in [Sun et al., 2015a], the incorrect equilibrium formation illustrated in Figure 8.3 is a saddle point. Such an equilibrium point is unstable for both single-integrator formation systems and double-integrator formation systems (see Theorem 20). However, this simulation also shows one advantage of using double-integrator formation models against 

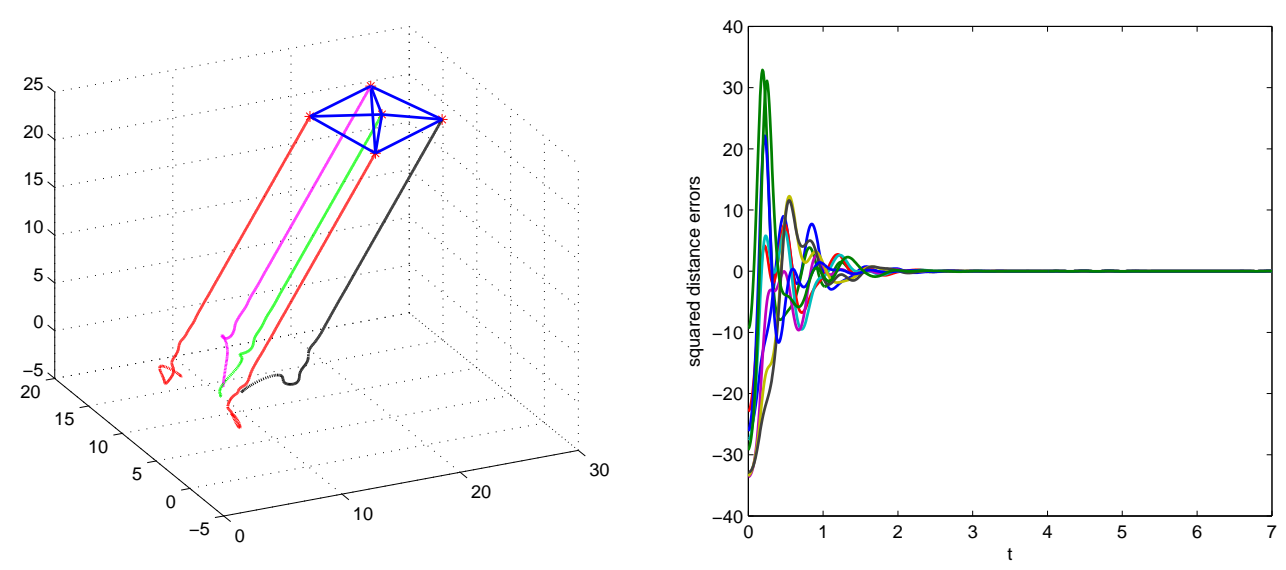

Figure 8.2: Simulation on formation flocking control of a double tetrahedron formation in the 3-D space with double-integrator systems.

single-integrator formation models. For double-integrator formation systems, even if one chooses initial positions that live in a lower-dimensional space, the formation system will not always live in that degenerate space. That is, double-integrator formation systems can escape from degenerate positions (e.g., collinear positions or coplanar positions) and thus will avoid the convergence to an incorrect degenerate equilibrium even if agents' positions are initially placed in a lower dimensional space. This is due to the fact that the rank-preserving property does not hold for rigid formation systems modelled by double-integrator dynamics.

\subsection{Concluding remarks}

In this chapter, we have considered formation control systems modelled by double integrators, which include the formation stabilization model and flocking control model. Due to the multi-equilibrium property caused by the nonlinear formation controller, a complete analysis of the convergence is quite challenging. This chapter serves as a further step to understand the dynamical behavior of such formation control systems. Novel properties of the system dynamics and convergence analysis for different equilibria are discussed by analyzing certain properties of the linearized systems and a parameterized Hamiltonian-like system. Some key results are summarized as follows:

- We discuss the measurement requirement for individual agents and establish the independence of a global coordinate frame for implementing the formation controller.

- Certain properties of the Jacobian matrix (e.g. the rank, null spaces and eigenvalues) for double-integrator formation systems are characterized. 

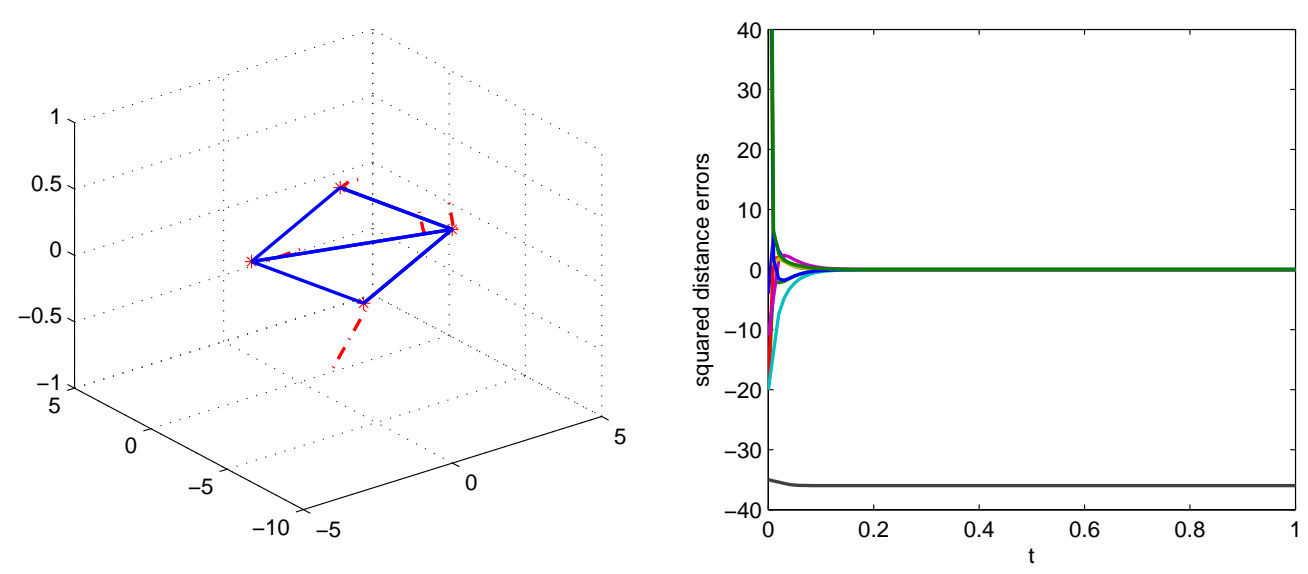

Figure 8.3: Simulation on formation shape control of a double tetrahedron formation in the 3-D space with single-integrator systems. The initial conditions are chosen to be in a plane in the 3-D space, and the formation converges to an incorrect formation shape that lives in that plane (i.e. an incorrect planar equilibrium formation).
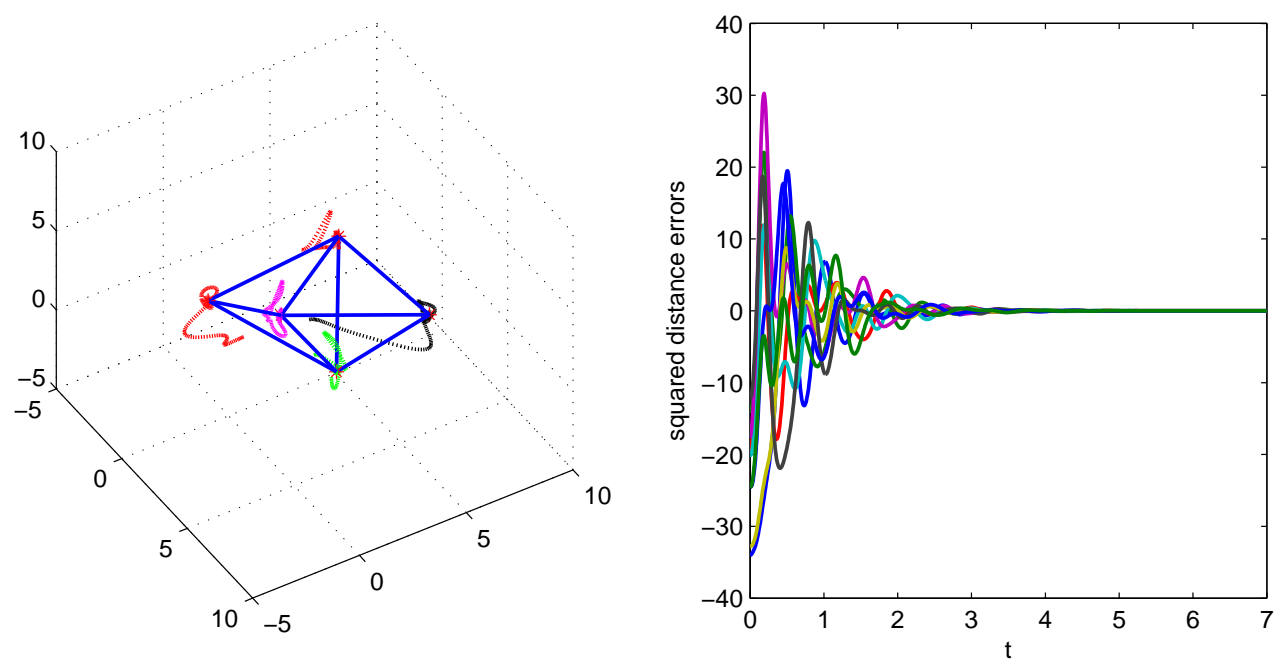

Figure 8.4: Simulation on formation shape control of a double tetrahedron formation in the 3-D space with double-integrator systems. The formation converges to a correct formation shape, even if one chooses degenerate coplanar initial positions. 
- Invariance principles concerning the equilibrium set and local stability are established for a family of parameterized Hamiltonian-like system, which builds the link between single-integrator formation systems and double-integrator formation systems. Thus, available results on equilibrium analysis in singleintegrator rigid formation systems reported in the vast literature can be extended to double-integrator formation systems. Note that the rank-preserving property, as discussed in Chapter 3 for single-integrator formation systems, does not hold for double-integrator formation systems.

- Several criteria for determining the stability and convergence of equilibrium sets for double-integrator formation systems are proposed. 


\section{Formation feasibility and motion generation of networked heterogeneous systems}

\section{Chapter summary}

In this chapter, we discuss a general problem of formation feasibility for multi-agent coordination control when individual agents have kinematics constraints modelled by affine nonlinear control systems with possible drift terms. Such dynamics models include the single-integrator model and double-integrator model considered in previous chapters, as well as other commonly-used models such as unicycle models discussed in many papers. In this problem, all agents need to work cooperatively to maintain a global formation task described by edge constraints. For such a multiagent group, we assume that different agents may have totally different dynamics, which brings the problem of coordination control of networked heterogeneous systems. Based on the concepts of (affine) distribution and codistribution, we propose a unified framework and an algebraic condition to determine the existence of feasible motions under both kinematic constraints and formation constraints. In the case that feasible motions exist, we propose a systematic procedure to obtain an equivalent dynamical system which generates all types of feasible motions. Several examples involving coordination control of constant-speed agents and heterogeneous agents are provided to demonstrate the application of this coordination control framework.

\subsection{Introduction}

\subsubsection{Background, motivation and related work}

Collective coordination control of networked multi-agent systems has received considerable attention in recent years, partly motivated by its applications in many areas [Cao et al., 2013; Knorn et al., 2016]. A particular class of cooperative tasks for multiagent coordination is formation control, in which the control objective is to form or maintain a prescribed geometric relationship for a group of spatially distributed 
agents [Oh et al., 2015]. Maintaining a formation is important in multi-agent coordination and in some cases would be a prerequisite for agents to perform additional tasks such as surveillance, coverage, target detection, etc ([Egerstedt and $\mathrm{Hu}, 2001$; Mesbahi and Egerstedt, 2010]).

Given a predefined formation task assigned to a group of distributed agents, a fundamental problem is to determine whether there exists a feasible trajectory for such an agent group to maintain the formation task subject to both kinematics and formation constraints. Formation feasibility was firstly discussed by P. Tabuada et. al. in [Tabuada et al., 2005]. They employed tools in differential geometry and derived an elegant criterion to analyze whether a networked agent group has feasible motions to maintain the formation constraint. Later papers along this direction include [Maithripala et al., 2008], which proposed a similar geometric approach to discuss some real-time formation control problems, including radar deception, formation keeping and formation reconfiguration. As revealed in [Tabuada et al., 2005] and [Maithripala et al., 2008], the central idea in the formation feasibility analysis is the interplay of agents' kinematic constraints and formation task constraints in controlling a formation and in generating a feasible trajectory for all the agents.

The concept of formation feasibility in this chapter builds on the analysis in [Tabuada et al., 2005], while here we present several extensions and generalizations to this fundamental networked control problem. First, in contrast to the control system model discussed in [Tabuada et al., 2005], in this chapter we consider agents' dynamics modelled by affine nonlinear control systems with possibly additional drift terms. This is motivated by the fact that most real-life control systems have drift terms. We note that affine nonlinear control systems with drift terms are very general in system modelling and are also popular choices for nonlinear control system analysis in the control community [Isidori, 1995]. One motivating example to consider agent dynamics with drift terms is the coordination control of constant-speed agents. Coordination control and collective circular motion with unit-speed agents or non-identical constant-speed agents was reported in e. g. [Sepulchre et al., 2008], [Seyboth et al., 2014]. More recently, the tracking control problem for multiple constant-speed agents was discussed in [Sun et al., 2015c] which also revealed the performance limitations in this coordination control problem. However, a general analysis on performance feasibility and its relationship to the speed constraint is still lacking. The results in this chapter will shed new insights to this coordination control problem.

Secondly, we further consider the case that individual agents in the networked system may have totally different dynamics, a possibility which includes fully actuated agents, under-actuated agents, or agents with a nonholonomic constraint and/or drift terms. Under this framework, we develop a fairly checkable condition for the existence of feasible motions by including both heterogeneous agent dynamics and formation constraints. Thus, the coordination control framework developed in this chapter is general enough to encompass many coordination control problems and presents a unified approach in the coordination feasibility analysis of networked heterogeneous systems.

When the feasibility condition is satisfied and there exist feasible motions, a nat- 
ural problem arises as how to obtain such feasible motions. We then propose a systematic approach to derive feasible motions in terms of an equivalent control system. In the later part of this chapter, we will also provide typical examples and detailed analysis involving coordination control of constant-speed agents and heterogeneous agents to demonstrate the application of this coordination control framework.

\subsubsection{Chapter organization}

The rest of this chapter is organized as follows. Section 9.2 introduces preliminary concepts on differential geometry and the problem formulation with a motivating example. Section 9.3 presents the main result on formation feasibility condition by incorporating both heterogeneous kinematic constraint and formation constraint into a unified form. Section 9.4 discusses its application in coordination control of networked agents with constant-speed dynamics. In Section 9.5, we discuss the motion generation problem using the formation feasibility analysis, together with a detailed illustrative example on cooperative control of networked heterogeneous agents. Finally, Section 9.6 concludes this chapter.

\subsection{Preliminaries, problem formulation and motivating ex- amples}

\subsubsection{Preliminaries on differential geometry and system equation}

Some standard notions from differential geometry (especially the concepts of distribution/codistribution) will be introduced in this subsection. More background can be found in [Isidori, 1995, Chapter 1] and [Murray et al., 1994, Chapter 7].

A distribution $\Delta(x)$ on $\mathbb{R}^{n}$ is an assignment of a linear subspace of $\mathbb{R}^{n}$ at each point $x$. Given a set of $k$ vector fields $X_{1}(x), X_{2}(x), \cdots, X_{k}(x)$, we define the distribution as

$$
\Delta(x)=\operatorname{span}\left\{X_{1}(x), X_{2}(x), \cdots, X_{k}(x)\right\} .
$$

A vector field $X$ belongs to a distribution $\Delta$ if $X(x) \in \Delta(x), \forall x \in \mathbb{R}^{n}$. Here we assume all distributions have constant rank.

A codistribution assigns a subspace to the dual space, denoted by $\left(\mathbb{R}^{n}\right)^{\star}$. Given a distribution $\Delta$, for each $x$ consider the annihilator of $\Delta$, which is the set of all covectors that annihilates all vectors in $\Delta(x)$ (see [Isidori, 1995, Chapter 1])

$$
\Delta^{\perp}=\left\{\omega \in\left(\mathbb{R}^{n}\right)^{\star} \mid\langle\omega, X\rangle=0, \forall X \in \Delta\right\}
$$

In this chapter, we assume that each individual agent's dynamics are described by the following general form (i.e. affine nonlinear control system)

$$
\dot{p}_{i}=f_{i, 0}+\sum_{j=1}^{l} f_{i, j} u_{i, j}
$$


where $p_{i} \in \mathbb{R}^{n_{i}}$ is the state of agent $i, n_{i}$ is the dimension of state space for agent $i, f_{i, 0}$ is a smooth drift term, and $u_{i, j}$ is the scalar control input associated with the smooth vector field $f_{i, j}$, and $l$ is the number of vector field functions. Such an affine nonlinear control system (9.1) with a drift term ${ }^{1}$ is a very general tool to describe many different types of real-life control systems, including control systems with an underactuation property or nonholonomic constraints.

\subsubsection{Motivating examples}

The paper [Tabuada et al., 2005] introduced the concept of motion feasibility problem for multi-agent formations. The discussions in [Tabuada et al., 2005] were restricted to the coordination control of drift-free control systems (i.e. $f_{i, 0}=0$ ) in the form of

$$
\dot{p}_{i}=\sum_{j=1}^{l} f_{i, j} u_{i, j}
$$

However, the above model is not general enough to describe many real-life nonlinear control systems. In contrast, the control system model in (9.1) encompasses a larger number of practical models and is modelling the most popular nonlinear control system [Isidori, 1995]. As an example, the unicycle-type agent with constantspeed constraints is one such nonlinear control system with drift terms that can be described by (9.1) but not by (9.2). Such system dynamics can be described as

$$
\begin{aligned}
\dot{x}_{i} & =v_{i} \cos \left(\theta_{i}\right) \\
\dot{y}_{i} & =v_{i} \sin \left(\theta_{i}\right) \\
\dot{\theta}_{i} & =u_{i}
\end{aligned}
$$

where $x_{i} \in \mathbb{R}, y_{i} \in \mathbb{R}$ are the coordinates in the real plane and $\theta_{i}$ is the heading angle for agent $i$. The agent has a fixed cruising speed $v_{i}>0$, which could be different for distinct agents; $u_{i}$ is the control input to be designed for steering the orientation.

Introducing the vector fields as

$$
f_{i, 0}=\left[\begin{array}{c}
v_{i} \cos \left(\theta_{i}\right) \\
v_{i} \sin \left(\theta_{i}\right) \\
0
\end{array}\right], f_{i, 1}=\left[\begin{array}{l}
0 \\
0 \\
1
\end{array}\right],
$$

we can rewrite the system (9.3) as

$$
\dot{p}_{i}=\left[\dot{x}_{i}, \dot{y}_{i}, \dot{\theta}_{i}\right]^{\top}=f_{i, 0}+f_{i, 1} u_{i}
$$

which has the form of (9.1).

\footnotetext{
${ }^{1}$ A statement by Roger Brockett in his recent survey paper [Brockett, 2014]: “almost all real systems have a drift term...".
} 


\subsubsection{Problem formulation: formation feasibility with kinematics and for- mation constraints}

In this chapter, we assume a networked multi-agent control system modelled by an undirected graph $\mathcal{G}$, in which we use $V$ to denote its vertex set and $E$ to denote the edge set. The vertices consist of $n$ heterogeneous agents each modelled by the general dynamical equation (9.1). The graph consists of $m$ edges, each with an inter-agent formation constraint.

A family of formation constraints $C$ is indexed by the edge set, denoted as $C_{E}=\left\{c_{i j}\right\}_{\left(v_{i}, v_{j}\right)}$ with $\left(v_{i}, v_{j}\right) \in E$. For each edge $\left(v_{i}, v_{j}\right), c_{i j}$ is a vector function defining the formation constraints between agents $i$ and $j$ and the constraint is enforced if $c_{i j}\left(p_{i}, p_{j}\right)=0$. Such formation constraints can be used to describe very general coordinate control problems, such as formation shape control, formation tracking, coverage control, etc. For example, in formation shape control, the constraint vector function $c_{i j}$ can be a function of desired relative position, or desired bearings, or desired distances between agents $i$ and $j$ that can be used to describe a target formation (for example, see [Oh et al., 2015]).

The formation feasibility problem is stated as follows:

Problem 1. Given a formation graph $F=[V, E, C]$, determine whether there are feasible trajectories $p_{i}(t)$ (or equivalently, feasible agents' motions $\dot{p}_{i}(t)$ ) for all agents whose kinematics are modelled by (9.1) with possible drift terms, such that the trajectories $p_{i}(t)$ also meet formation constraints $C_{E}=\mathbf{0}$, where $C_{E}=\left[\cdots, c_{i j}^{\top}, \cdots\right]^{\top}$ for all $\left(v_{i}, v_{j}\right) \in E$ and $t \in I$ where $I$ is a specified time interval.

In the case that there exist feasible motions, we further consider the motion generation problem formulated as below.

Problem 2. Given a formation graph $F=[V, E, C]$ with feasible agents' motions, determine an equivalent control system that generates feasible motions for the networked heterogeneous multi-agent system.

Remark 27. A prerequisite of solving the above motion feasibility problem is that the formation constraint for the distributed edge set should be non-conflicting, and the overall constraint for all the edges should be realizable at least in the full Euclidean space. For example, if the formation is described by relative position vectors which are conflicting, then the formation is unachievable and this may lead to unexpected flocking motion [Dimarogonas and Kyriakopoulos, 2008]. As another example, if the formation is described by distance constraints to realize a rigid shape in a 3-agent group, then the set of formation distances should satisfy the triangle inequality [Sun et al., 2014c]. Thus, in order to well define the feasibility problem, we need to first assume that the formation constraints are non-conflicting and realizable. 


\subsection{Conditions for feasible motion}

\subsubsection{Constraints on inter-agent formations}

For ease of notation, we collect all of the agents' states together, denoting them by the composite state vector $P=\left[p_{1}^{\top}, p_{2}^{\top}, \cdots, p_{n}^{\top}\right]^{\top}$. We collect the formation constraints for all the edges and define an overall formation constraint denoted by $C_{E}=\left[\cdots, c_{i j}^{\top}, \cdots\right]^{\top}=\mathbf{0}$. Formation feasibility means that the constraints are satisfied along the formation trajectories. Thus, one can obtain

$$
\frac{\mathrm{d}}{\mathrm{d} t} C_{E}=\mathcal{L}_{P} C_{E}+\frac{\partial C_{E}}{\partial t}=0
$$

where $\mathcal{L}_{P} C_{E}$ denotes the Lie derivative of $C_{E}$ along $P$.

If we group all the constraints for all the edges by writing down a compact form $T_{F}=-\left[\cdots,\left(\frac{\partial c_{i j}}{\partial t}\right)^{\top}, \cdots\right]^{\top}$ and $\mathcal{L}_{P} C_{E}=: \Omega_{F}(\dot{P})$ with the duality product relationship between the rows of $\Omega_{F}$ and $\dot{P}$, we can reexpress equation (9.6) as

$$
\Omega_{F}(\dot{P})=T_{F}
$$

where the subscript $F$ stands for formation. Thus, the vector field $\dot{P}$ defined by the above equation represents possible motions for all the agents that respect the formation constraint.

\subsubsection{Constraints on individual agents' kinematics}

The kinematics constraints of the drift-free agent model (9.2) can be described in an equivalent form

$$
\omega_{i, j}\left(p_{i}\right) \dot{p}_{i}=0, j=1, \cdots, n_{i}-l .
$$

The above transformation is based on the idea that a distribution generated by vector fields of a nonlinear control system can be equivalently defined by its annihilating codistribution. Note that each $\omega_{i, j}\left(p_{i}\right)$ in (9.8) is a row covector in the dual space $\left(\mathbb{R}^{n_{i}}\right)^{\star}$.

We consider a group of $n$ heterogeneous agents, each modelled by the general control system in the form of (9.1). Note that an affine nonlinear control system (9.1) with drifts can be equivalently described by the following affine distribution

$$
\Delta_{i}=f_{i, 0}+\operatorname{span}\left\{f_{i, 1}, f_{i, 2}, \cdots, f_{i, l}\right\}
$$

As opposed to the system constraint transformation shown in (9.8) for drift-free systems, for the system (9.1) with drifts, one can obtain a corresponding transformation with equivalent constraints via the construction of covectors:

$$
\omega_{i, j}\left(p_{i}\right) \dot{p}_{i}=q_{i, j}, j=1, \cdots, n_{i}-l,
$$


where the parameter $q_{i, j}$ is due to the existence of the drift term $f_{i, 0}$. We collect all the row covectors $\omega_{i, j}$ as

$$
\Omega_{K_{i}}=\left[\begin{array}{c}
\omega_{i, 1} \\
\omega_{i, 2} \\
\vdots \\
\omega_{i, n_{i}-l}
\end{array}\right]
$$

and similarly define $T_{K_{i}}=\left[q_{i, 1}, q_{i, 2}, \cdots, q_{i, n_{i}-l}\right]^{\top}$. By doing this, one can rewrite (9.10) in a compact form as follows

$$
\Omega_{K_{i}} \dot{p}_{i}=T_{K_{i}}
$$

where the subscript $K$ stands for kinematics. From the above, we collect all the kinematic constraints for all the agents in a composite form

$$
\Omega_{K}=\left[\Omega_{K_{1}}^{\top}, \Omega_{K_{2}}^{\top}, \cdots, \Omega_{K_{n}}^{\top}\right]^{\top}, T_{K}=\left[T_{K_{1}}^{\top}, T_{K_{2}}^{\top}, \cdots, T_{K_{n}}^{\top}\right]^{\top}
$$

and thus the overall kinematic constraint for all the agents can be stated in a compact equation $\Omega_{K}(\dot{P})=T_{K}$.

Remark 28. Note that if the system $i$ has a drift (as in (9.1)) but the drift satisfies

$$
f_{i, 0} \in \operatorname{span}\left\{f_{i, 1}, f_{i, 2}, \cdots, f_{i, l}\right\}
$$

then one can choose a control $u_{i}$ to cancel the drift. In such case the system with drifts (9.1) can be transformed to be the drift-free system (9.2), and the codistribution can be defined by using the covector $\omega_{i, j}$ in (9.8). Note that the constant-speed agent model (9.3) does not meet the above assumption (9.13).

\subsubsection{Main results on formation feasibility}

By including both kinematic constraints and formation constraints for all the agents into a unified expression, the formation feasibility problem then becomes one of establishing existence of a solution to the following equation

$$
\Omega(\dot{P})=T
$$

where

$$
\Omega=\left[\begin{array}{l}
\Omega_{F} \\
\Omega_{K}
\end{array}\right], T=\left[\begin{array}{c}
T_{F} \\
T_{K}
\end{array}\right]
$$

We remark that the expression of the codistribution $\Omega$ is coordinate-free and is also independent of the enumeration of edge sets.

The formation feasibility condition is shown in the following theorem. 
Theorem 22. The undirected formation has feasible motions if the above equation (9.14) has solutions, or equivalently if $T$ belongs to the range of $\Omega$.

Remark 29. As we remarked before, the condition is applicable for networked heterogeneous systems as the model of (9.1) includes different types of agent dynamics. Also, to determine and calculate the condition, symbolic toolboxes from certain popular software (e.g. Matlab or Mathematica) are readily available. Note that the condition stated in (9.14) of Theorem 22 can be reexpressed in an equivalent way in terms of the rank condition of the form $\operatorname{rank}(\Omega)=\operatorname{rank}([\Omega, T])$. This alternative condition could facilitate calculations using available softwares.

Remark 30. For the time-invariant formation case we have $\frac{\partial C_{E}}{\partial t}=0$ and thus $T_{F}=\mathbf{0}$; in the drift-free model case there holds $T_{K, i}=0$ for all $i$ and thus $T_{K}=\mathbf{0}$. Under these two special cases, one has $T=\left[\begin{array}{l}T_{F} \\ T_{K}\end{array}\right]=\mathbf{0}$, and the feasibility condition in Theorem 22 reduces to the condition statement in Theorem 4.1 of [Tabuada et al., 2005].

\subsection{Application to coordination control of multiple constant- speed agents}

Coordination and formation control of constant-speed agents (e.g. fixed-wing UAVs with constant cruising speeds) has been discussed in [Sepulchre et al., 2008], [Anderson et al., 2008a], [Seyboth et al., 2014], [Guler et al., 2013], [Sun et al., 2015c], etc. However, a complete solution to such a control problem is not yet available. In this section, this coordination control problem is revisited with insights obtained from the above formation feasibility analysis.

\subsubsection{Affine distribution and codistribution generated by constant-speed dynamics}

Following similar notations as in Section 9.2, we denote each agent's state as $p_{i}=$ $\left[x_{i}, y_{i}, \theta_{i}\right]^{\top}$, with the system dynamics described in (9.3) and (9.5). The vector fields shown in (9.4) generate the following affine distribution:

$$
\Delta_{i}=f_{i, 0}+\operatorname{span}\left(f_{i, 1}\right)
$$

We now construct the covectors for the codistribution ${ }^{2} \Delta_{i}^{\perp}$. Note that the dimension of the affine distribution $\Delta_{i}$ is 1 , which implies that the dimension of the codistribution is 2 . We denote two linearly independent covectors of the codistribution as $\omega_{i, 1}$ and $\omega_{i, 2}$. Direct calculation shows the following:

$$
\left[\begin{array}{ccc}
\sin \left(\theta_{i}\right) & -\cos \left(\theta_{i}\right) & 0 \\
\cos \left(\theta_{i}\right) & \sin \left(\theta_{i}\right) & 0
\end{array}\right]\left[\begin{array}{c}
\dot{x}_{i} \\
\dot{y}_{i} \\
\dot{\theta}_{i}
\end{array}\right]=\left[\begin{array}{c}
0 \\
v_{i}
\end{array}\right]
$$

\footnotetext{
${ }^{2}$ The codistribution that is dual to an affine distribution is termed as t-codistribution in [Elkin, 2012].
} 
which is an instance of system constraint transformation shown in (9.10). Thus, according to (9.12), we let $T_{K_{i}}=\left[q_{i, 1}, q_{i, 2}\right]^{\top}=\left[0, v_{i}\right]^{\top}$ in the construction of the corresponding codistribution. Based on the constraint transformation concept presented in Section 9.3.2, the above (9.17) presents an equivalent way to describe the kinematics constraint for a constant-speed agent. Note that a basis for describing any covector in the dual space is $\left(\mathrm{d} x_{i}, \mathrm{~d} y_{i}, \mathrm{~d} \theta_{i}\right)$, which gives the following expression of the covectors

$$
\begin{aligned}
& \omega_{i, 1}=\sin \left(\theta_{i}\right) \mathrm{d} x_{i}-\cos \left(\theta_{i}\right) \mathrm{d} y_{i}, \\
& \omega_{i, 2}=\cos \left(\theta_{i}\right) \mathrm{d} x_{i}+\sin \left(\theta_{i}\right) \mathrm{d} y_{i} .
\end{aligned}
$$

By denoting $\Omega_{K, i}=\left[\begin{array}{c}\omega_{i, 1} \\ \omega_{i, 2}\end{array}\right]$, there holds $\Omega_{K, i} f_{i, 1}=0$ and $\Omega_{K, i} f_{i, 0}=T_{K_{i}}$. These covectors and their equivalent forms in formulating distributions/codistributions will be useful in later analysis on coordination control with constant-speed agent groups.

\subsubsection{Example: rigid formation maintenance and coordination by constant- speed agents}

In this example, we suppose the formation is defined by a certain set of inter-agent distances (as the problem formulation in [Krick et al., 2009]) and the control task is to coordinate a group of constant-speed agents while maintaining the rigid target formation. We consider a three-agent group in this control task. The inter-agent distances are denoted as $d_{i j}$ with $i, j=1,2,3, i \neq j$, and the constraint function for edge $(i, j)$ is defined as $c_{i j}=\frac{1}{2}\left(x_{i}-x_{j}\right)^{2}+\frac{1}{2}\left(y_{i}-y_{j}\right)^{2}-d_{i j}^{2}$.

According to the analysis in Section 9.3.1 and Section 9.4.1, the matrices $\Omega_{F}$ and $\Omega_{K}$ for characterizing codistributions can be calculated as shown in (9.18) and (9.19), respectively. ${ }^{3}$ The overall constraint matrix $\Omega$ is now constructed as $\Omega=$ $\left[\Omega_{F}^{\top}, \Omega_{K}^{\top}\right]^{\top} \in \mathbb{R}^{9 \times 9}$.

$$
\begin{gathered}
\Omega_{K}=\left[\begin{array}{c}
\sin \left(\theta_{1}\right) \mathrm{d} x_{1}-\cos \left(\theta_{1}\right) \mathrm{d} y_{1} \\
\cos \left(\theta_{1}\right) \mathrm{d} x_{1}+\sin \left(\theta_{1}\right) \mathrm{d} y_{1} \\
\sin \left(\theta_{2}\right) \mathrm{d} x_{2}-\cos \left(\theta_{2}\right) \mathrm{d} y_{2} \\
\cos \left(\theta_{2}\right) \mathrm{d} x_{2}+\sin \left(\theta_{2}\right) \mathrm{d} y_{2} \\
\sin \left(\theta_{3}\right) \mathrm{d} x_{3}-\cos \left(\theta_{3}\right) \mathrm{d} y_{3} \\
\cos \left(\theta_{3}\right) \mathrm{d} x_{3}+\sin \left(\theta_{3}\right) \mathrm{d} y_{3}
\end{array}\right] \\
\Omega_{F}=\left[\begin{array}{c}
\left(x_{1}-x_{2}\right) \mathrm{d} x_{1}+\left(y_{1}-y_{2}\right) \mathrm{d} y_{1}+\left(x_{2}-x_{1}\right) \mathrm{d} x_{2}+\left(y_{2}-y_{1}\right) \mathrm{d} y_{2} \\
\left(x_{1}-x_{3}\right) \mathrm{d} x_{1}+\left(y_{1}-y_{3}\right) \mathrm{d} y_{1}+\left(x_{3}-x_{1}\right) \mathrm{d} x_{3}+\left(y_{3}-y_{1}\right) \mathrm{d} y_{3} \\
\left(x_{2}-x_{3}\right) \mathrm{d} x_{2}+\left(y_{2}-y_{3}\right) \mathrm{d} y_{2}+\left(x_{3}-x_{2}\right) \mathrm{d} x_{3}+\left(y_{3}-y_{2}\right) \mathrm{d} y_{3}
\end{array}\right]
\end{gathered}
$$

\footnotetext{
${ }^{3}$ We would like to emphasize that $\Omega_{F}$ and $\Omega_{K}$ are matrices and not vectors, with $\Omega_{F} \in \mathbb{R}^{6 \times 9}$ and $\Omega_{K} \in \mathbb{R}^{3 \times 9}$. Note that we have used the standard basis $\left(\mathrm{d} x_{1}, \mathrm{~d} y_{1}, \mathrm{~d} \theta_{1}, \cdots, \mathrm{d} x_{3}, \mathrm{~d} y_{3}, \mathrm{~d} \theta_{3}\right)$ in the dual space to write down each row covector in $\Omega_{F}$ and $\Omega_{K}$.
} 


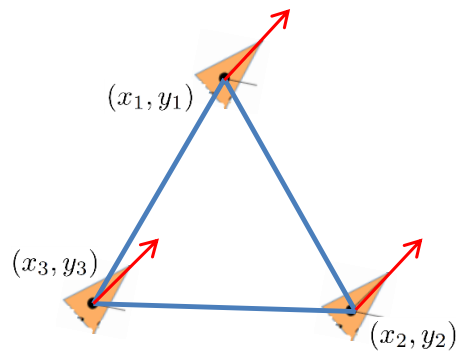

(a) A translational motion with the same direction

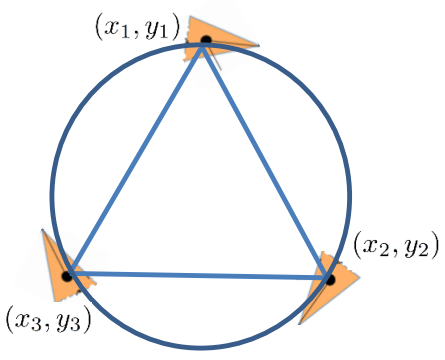

(b) A rotational motion around the triangle centroid

Figure 9.1: Two feasible formations with a group of constant-speed agents.

$$
R=\left[\begin{array}{cccccc}
\left(x_{1}-x_{2}\right) & \left(y_{1}-y_{2}\right) & \left(x_{2}-x_{1}\right) & \left(y_{2}-y_{1}\right) & 0 & 0 \\
\left(x_{1}-x_{3}\right) & \left(y_{1}-y_{3}\right) & 0 & 0 & \left(x_{3}-x_{1}\right) & \left(y_{3}-y_{1}\right) \\
0 & 0 & \left(x_{2}-x_{3}\right) & \left(y_{2}-y_{3}\right) & \left(x_{3}-x_{2}\right) & \left(y_{3}-y_{2}\right)
\end{array}\right]
$$

The matrix $T$ is calculated as $T=\left[0, v_{1}, 0, v_{2}, 0, v_{3}, 0,0,0\right]^{\top} \in \mathbb{R}^{9 \times 1}$. Thus, the formation feasibility problem is now transformed to the problem of determining whether there exists a vector field $\dot{P}$ that satisfies $\Omega \dot{P}=T$. After these matrices are constructed with the specified parameters $v_{i}$ and $d_{i j}$ describing both kinematics constraints and formation constraints, available computation approaches could then be employed to assist the analysis of determining the solution issue.

For the aim of demonstration, in the following analysis we assume that $d_{12}=$ $d_{23}=d_{31}=\beta$, that is, the target rigid formation is an equilateral triangle. We then consider different sets of cruising speeds to determine the solution property. In the case that all agents have identical cruising speeds, i.e. $v_{1}=v_{2}=v_{3}$, a simple calculation shows that the motion solution is that either (i) $\dot{\theta}_{1}=\dot{\theta}_{2}=\dot{\theta}_{3}=0, \theta_{1}=\theta_{2}=\theta_{3}$, or (ii) $\dot{\theta}_{1}=\dot{\theta}_{2}=\dot{\theta}_{3}, \theta_{1}=\theta_{2}+\frac{2 \pi}{3}=\theta_{3}+\frac{4 \pi}{3}$, which correspond to (i) a translational motion with the same direction or (ii) a rotational motion around the triangle centroid. In the case that $v_{1}=0, v_{2}=v_{3}$, a feasible motion exists such that agents 2 and 3 rotate around agent 1 with the same angular velocity. These two types of feasible formations are shown in Figure 9.1. In the case that all agents have non-identical cruising speeds, there usually does not exist a solution to the feasibility condition except for some special cases, which agrees with our intuition that maintaining a rigid shape by using non-identical constant-speed agents is generally impossible.

We also remark that the codistribution matrix $\Omega_{F}$ for characterizing the formation constraints can be interpreted as a rigidity matrix $R$ (see definitions in Section 2.3.2 of Chapter 2). For ease of demonstration, $R$ is written in (9.20). A reinterpretation of the above formation feasibility result is that any feasible velocity $\left(\dot{x}_{i}, \dot{y}_{i}\right)$ should satisfy $\left[\dot{x}_{1}, \dot{y}_{1}, \dot{x}_{2}, \dot{y}_{2}, \dot{x}_{3}, \dot{y}_{3}\right]^{\top} \in \operatorname{null}(R)$, where $\operatorname{null}(R)$ denotes the null space of the rigidity matrix $R$ associated with a realization of the target formation (see Section 6.2.1 of 
Chapter 6 for detailed discussions on their null spaces). For a 2-D infinitesimally rigid formation, the dimension of the null space of the associated rigidity matrix $R$ is 3 (see Theorem 1 in Chapter 2), corresponding to rotational and translational motions. Thus, the motion solution of a feasible translational motion mentioned above is that $\left[\dot{x}_{1}, \dot{y}_{1}, \dot{x}_{2}, \dot{y}_{2}, \dot{x}_{3}, \dot{y}_{3}\right]^{\top} \in \operatorname{span}\left(\mathbf{1} \otimes I_{2}\right)$, and the feasible rotational motion corresponds to the rotational part in the null space of $R$. This gives another viewpoint to confirm the above analysis.

\subsection{Motion generation and coordination control with hetero- geneous agents}

\subsubsection{Generalization and motion derivation}

We mention the fact that the theory developed in the previous sections is not limited to any specific type of agent dynamics, and it provides a unified analysis and framework to the coordination control with distinct agent dynamics. Actually, agent dynamics considered here include, but are not limited to, the following types:

- fully actuated agents;

- agents with drift-free, nonholonomic dynamics;

- agents modelled by affine systems with possible drift terms.

Thus, the general framework developed in previous sections can be applied in the coordination control problem for a multi-agent group with heterogeneous dynamics. We then consider Problem 2, i.e., how to derive feasible motions for networked heterogeneous agents.

Suppose the formation feasibility condition is satisfied, i.e. there holds $\operatorname{rank}(\Omega)=$ $\operatorname{rank}([\Omega, T])$. One can first calculate a special solution to the feasibility equation (9.14), denoted by $\bar{K}$. Further we suppose the null space of $\Omega$ is spanned by $\kappa$ vectors, denoted by $K_{1}, K_{2}, \cdots, K_{\kappa}$. Then any feasible motion for the overall heterogeneous networked system can be generated by the following equivalent dynamical system

$$
\dot{P}=\bar{K}+\sum_{l=1}^{\kappa} K_{l} w_{l}
$$

where $w_{l}$ is an input that activates the associated vector field $K_{l}$.

\subsubsection{Illustrative example: coordination control with a group of heteroge- neous agents}

In this subsection, by using an illustrative example with a group of heterogeneous agents, we show how to apply the previous results to determine whether the networked system has a feasible motion under both kinematic and formation constraints, and if there exist feasible motions, how to generate them. 
The illustrative example on coordination control in this section is adopted from [Tabuada et al., 2005], but here we consider a more complicated problem when all agents are with heterogeneous dynamics. Consider an undirected formation consisting of three agents. We assume agent 1 is modelled by the dynamics (9.3) with both nonholonomic constraint and drift term, while agents 2 and 3 have totally different dynamics. To be specific, we assume that agent 2 is a unicycle-type robot with nonholonomic constraint and adjustable forward speed, whose system equation is written as

$$
\begin{aligned}
& \dot{x}_{2}=u_{2,1} \cos \left(\theta_{2}\right) \\
& \dot{y}_{2}=u_{2,1} \sin \left(\theta_{2}\right) \\
& \dot{\theta}_{2}=u_{2,2}
\end{aligned}
$$

or in an equivalent and compact form

$$
\dot{p}_{2}=\left[\dot{x}_{2}, \dot{y}_{2}, \dot{\theta}_{2}\right]^{\top}=f_{2,1} u_{2,1}+f_{2,2} u_{2,2}
$$

with

$$
f_{2,1}=\left[\begin{array}{c}
\cos \left(\theta_{2}\right) \\
\sin \left(\theta_{2}\right) \\
0
\end{array}\right], f_{2,2}=\left[\begin{array}{l}
0 \\
0 \\
1
\end{array}\right]
$$

We assume agent 3 is fully actuated, and its dynamical equation is described by single integrators

$$
\dot{x}_{3}=u_{3,1}, \quad \dot{y}_{3}=u_{3,2}
$$

where $u_{3,1}, u_{3,2}$ are the control inputs which generate velocity in the $x$ and $y$ coordinate.

For each individual agent, we first formulate the (affine) distribution and codistribution generated by its kinematic constraint. For agent 1, the affine distribution and codistribution can be obtained from the same procedure as in Section 9.4.1. For agent 2, one can show the distribution generated by its system vector fields is described by $\Delta_{2}=\operatorname{span}\left\{f_{2,1}, f_{2,2}\right\}$, which can be equivalently stated by the annihilating codistribution

$$
\Delta_{2}^{\perp}=\operatorname{span}\left\{\sin \left(\theta_{2}\right) \mathrm{d} x_{2}-\cos \left(\theta_{2}\right) \mathrm{d} y_{2}\right\} .
$$

For agent 3 , the two vectors $[1,0]^{\top}$ and $[0,1]^{\top}$ span the full $\mathbb{R}^{2}$ space and thus the codistribution is null. By putting together kinematic constraints for all the agents, 
one can obtain a compact form

$$
\Omega_{K}=\left[\begin{array}{c}
\omega_{1,1} \\
\omega_{1,2} \\
\sin \left(\theta_{2}\right) \mathrm{d} x_{2}-\cos \left(\theta_{2}\right) \mathrm{d} y_{2}
\end{array}\right]
$$

with $T_{K}=\left[0, v_{1}, 0\right]^{\top}$.

The formation constraint follows similarly from [Tabuada et al., 2005, Example 4.2], with some slight modifications. We assume that the formation constraint consists of a relative position constraint for the edge $(1,2)$, and a distance constraint for the edge $(1,3)$. Specifically, the constraint associated with the edge between agent 1 and agent 2 is defined by

$$
c_{12}=\left[\begin{array}{c}
x_{1}-x_{2}-\delta_{x} \\
y_{1}-y_{2}-\delta_{y} \\
\theta_{1}-\theta_{2}
\end{array}\right],
$$

where $\delta_{x}$ and $\delta_{y}$ are positive constants specifying the desired relative displacement between agent 1 and agent 2 . The constraint between agent 1 and agent 3 is defined by the function

$$
c_{13}=\frac{1}{2}\left(x_{1}-x_{3}\right)^{2}+\frac{1}{2}\left(y_{1}-y_{3}\right)^{2}-\delta
$$

where $\delta$ is a positive constant specifying the desired distance between agent 1 and agent 3 .

Up to now, it is not obvious whether there exists a feasible motion just from mere observation. The theory developed in Section 9.3.3 and Section 9.5.1 should be employed. The codistribution for characterizing the formation constraint can be described in (9.30).

$$
\Omega_{F}=\left[\begin{array}{c}
\mathrm{d} x_{1}-\mathrm{d} x_{2} \\
\mathrm{~d} y_{1}-\mathrm{d} y_{2} \\
\mathrm{~d} \theta_{1}-\mathrm{d} \theta_{2} \\
\left(x_{1}-x_{3}\right) \mathrm{d} x_{1}+\left(y_{1}-y_{3}\right) \mathrm{d} y_{1}+\left(x_{3}-x_{1}\right) \mathrm{d} x_{3} \\
+\left(y_{3}-y_{1}\right) \mathrm{d} y_{3}
\end{array}\right]
$$

where we have used the standard basis $\left\{\mathrm{d} x_{1}, \mathrm{~d} y_{1}, \mathrm{~d} \theta_{1}, \cdots, \mathrm{d} x_{3}, \mathrm{~d} y_{3}\right\}$ to describe each covector in $\Omega_{F}$. By grouping both the kinematic constraint and formation constraint, one can write down a composite constraint matrix $\Omega$ as shown in (9.31). Straightforward calculation shows that $\operatorname{rank}(\Omega)=\operatorname{rank}([\Omega, T])$ holds in this example. Thus, there exist feasible motions to coordinate such a heterogeneous agent group while maintaining the target formation. One special solution $\bar{K}$ to the equation $\Omega \dot{P}=T$ and the two null vectors $K_{1}, K_{2}$ of $\Omega$ are calculated in (9.32).

Then the feasible motion for the heterogeneous agent group can be described by 


$$
\begin{aligned}
\Omega & =\left[\begin{array}{cccccccc}
\sin \left(\theta_{1}\right) & -\cos \left(\theta_{1}\right) & 0 & 0 & 0 & 0 & 0 & 0 \\
\cos \left(\theta_{1}\right) & \sin \left(\theta_{1}\right) & 0 & 0 & 0 & 0 & 0 & 0 \\
0 & 0 & 0 & \sin \left(\theta_{2}\right) & -\cos \left(\theta_{2}\right) & 0 & 0 & 0 \\
1 & 0 & 0 & -1 & 0 & 0 & 0 & 0 \\
0 & 1 & 0 & 0 & -1 & 0 & 0 & 0 \\
0 & 0 & 1 & 0 & 0 & -1 & 0 & 0 \\
x_{1}-x_{3} & y_{1}-y_{3} & 0 & 0 & 0 & 0 & x_{3}-x_{1} & y_{3}-y_{1}
\end{array}\right] \\
\dot{P} & =\left[\begin{array}{llllllll}
\dot{x}_{1} & \dot{y}_{1} & \dot{\theta}_{1} & \dot{x}_{2} & \dot{y}_{2} & \dot{\theta}_{2} & \dot{x}_{3} & \dot{y}_{3}
\end{array}\right]^{\top}, \\
T & =\left[\begin{array}{llllllll}
0 & v_{1} & 0 & 0 & 0 & 0 & 0 & 0
\end{array}\right]^{\top}
\end{aligned}
$$

$$
\bar{K}=\left[\begin{array}{c}
v_{1} \cos \left(\theta_{1}\right) \\
v_{1} \sin \left(\theta_{1}\right) \\
0 \\
v_{1} \cos \left(\theta_{1}\right) \\
v_{1} \sin \left(\theta_{1}\right) \\
0 \\
v_{1} \cos \left(\theta_{1}\right) \\
v_{1} \sin \left(\theta_{1}\right)
\end{array}\right], K_{1}=\left[\begin{array}{c}
0 \\
0 \\
0 \\
0 \\
0 \\
0 \\
y_{3}-y_{1} \\
x_{1}-x_{3}
\end{array}\right], K_{2}=\left[\begin{array}{l}
0 \\
0 \\
1 \\
0 \\
0 \\
1 \\
0 \\
0
\end{array}\right]
$$

the following equivalent control system

$$
\dot{P}=\bar{K}+K_{1} w_{1}+K_{2} w_{2}
$$

The above abstracted control system provides certain insights on different types of feasible motions for the heterogeneous agent group according to different choices of $w_{1}$ and $w_{2}$. More precisely, all feasible motions can be categorized into the following four groups:

- Type (i): the case of $w_{1}=0$ and $w_{2}=0$ : all the three agents translate with the same heading while maintaining the formation constraints (9.28) and (9.29);

- Type (ii): the case of $w_{1} \neq 0$ and $w_{2}=0$ : agent 1 and agent 2 translate with the same heading, while agent 3 performs a combined motion of both translation and rotation generated by the dynamics $\dot{x}_{3}=v_{1} \cos \left(\theta_{1}\right)+y_{3}-y_{1}, \dot{y}_{3}=$ $v_{1} \sin \left(\theta_{1}\right)+x_{3}-x_{1}$

- Type (iii): the case of $w_{1}=0$ and $w_{2} \neq 0$ : all the three agents rotate with the same angular velocity, while agent 1 and agent 2 have the same instantaneous phase;

- Type (iv): the case of $w_{1} \neq 0$ and $w_{2} \neq 0$ : agent 1 and agent 2 rotate with the same angular velocity and have the same instantaneous phase, while agent 3 
performs a combined motion of both translation and rotation generated by the dynamics $\dot{x}_{3}=v_{1} \cos \left(\theta_{1}\right)+y_{3}-y_{1}, \dot{y}_{3}=v_{1} \sin \left(\theta_{1}\right)+x_{3}-x_{1}$.

Thus, by using the general motion generation system (9.33), all kinds of feasible motions can be derived. Figure 9.2 depicts all these four types of feasible motions and all agents' trajectories ${ }^{4}$.

\subsection{Concluding remarks}

In this chapter, we have discussed the formation feasibility problem in the coordination control of multi-agent systems whose dynamics are heterogeneous and may contain drift terms. Based on concepts from elementary differential geometry and tools of (affine) distribution and codistribution, we formulate the constraints from the formation aspect and kinematics aspect into a unified form, and propose an algebraic condition to determine the existence of feasible motions. Furthermore, in the case that the feasibility condition holds, we propose a systematic approach to obtain an equivalent dynamical system that can generate all feasible motions. Through several examples, we have shown how this general and unified framework can be applied to coordination control of constant-speed agent groups and heterogeneous agent groups.

\footnotetext{
${ }^{4}$ We performed numerical simulations via Matlab to generate all these figures. In the simulations we set $v_{1}=1, \delta_{x}=-2, \delta_{y}=-2, \delta=2$. The initial states for all agents were set as $\left[x_{1}(0), y_{1}(0), \theta_{1}(0)\right]^{\top}=$ $[0,1,2]^{\top},\left[x_{2}(0), y_{2}(0), \theta_{2}(0)\right]^{\top}=[2,3,2]^{\top}$ and $\left[x_{3}(0), y_{3}(0)\right]^{\top}=[0,3]^{\top}$. Note that at the initial states the formation constraint is satisfied.
} 

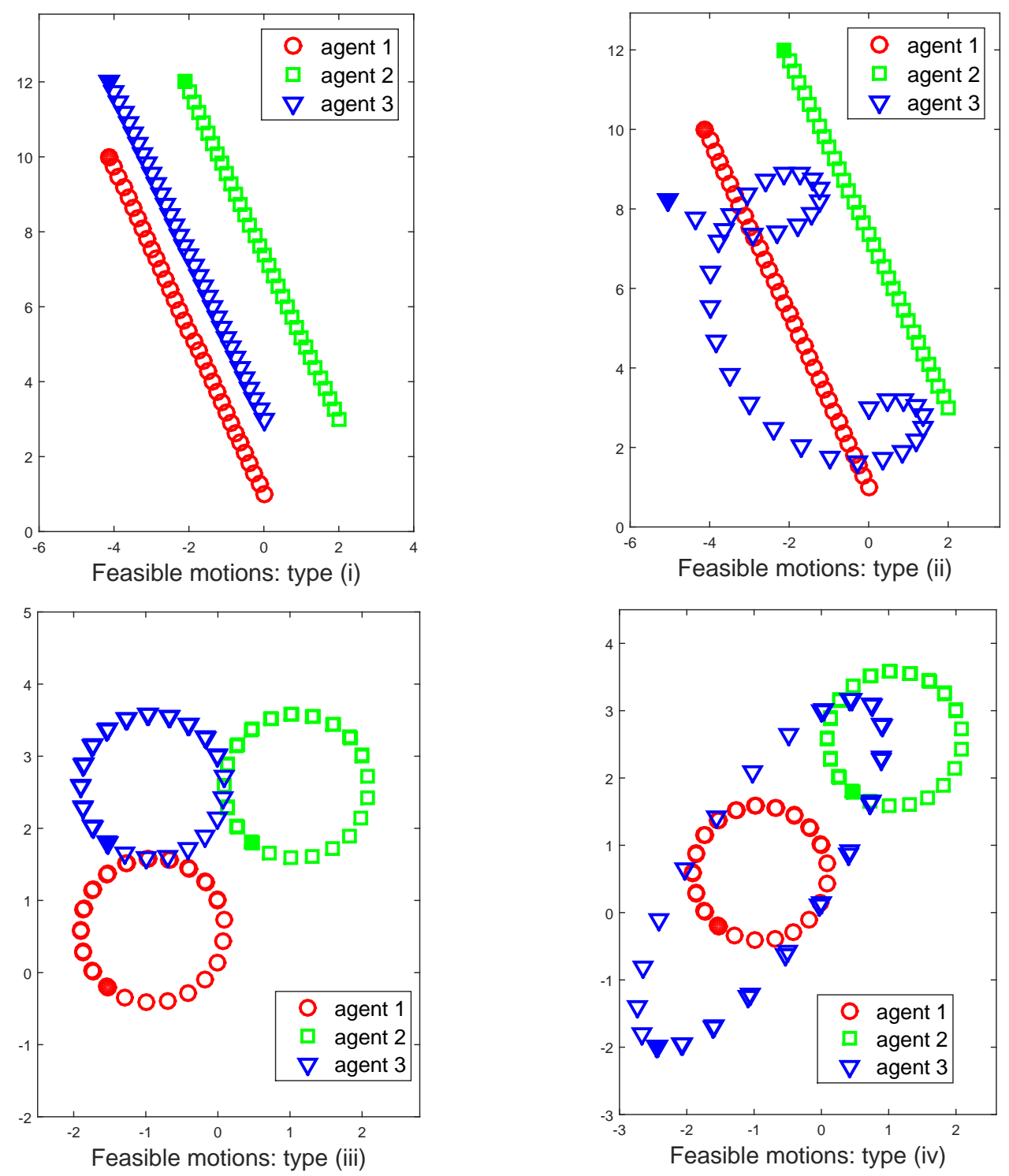

Figure 9.2: Feasible motions for heterogeneous agent group. Type (i): $w_{1}=0$ and $w_{2}=0$; Type (ii): $w_{1}=1$ and $w_{2}=0$; Type (iii): $w_{1}=0$ and $w_{2}=1$; Type (iv): $w_{1}=1$ and $w_{2}=1$. The solid markers indicate the final positions for all the agents. 


\section{Conclusions and future work}

By way of conclusion, the main contributions of this thesis are summarized in this chapter. Suggestions for possible future research directions are also outlined in Section 10.2.

\subsection{Thesis summary and contributions}

This thesis mainly considers the problem of distributed coordination and formation control for multi-agent systems. We have addressed a wide range of issues in this field, ranging from stability and equilibrium analysis of rigid formation systems, certain practical considerations in applying formation control algorithms (including mismatched distances, formation orientation stabilization and quantized measurements), different system models and their effects in distributed coordination and formation achievement ${ }^{1}$. We now show a brief summary of the main results and contributions of this thesis.

\subsubsection{Formation control systems: equilibrium and stability analysis}

- We have addressed some open problems in rigid formation control when the formation system is modelled by single-integrator systems. In Chapter 3, a rank-preserving property is established for rigid formation control systems, which reveals several novel insights on its equilibrium analysis. We have further proved the instability of a special equilibrium set called degenerate equilibria. On the one hand, some previously established results can be seen as special cases in this framework. On the other hand, we have provided a much simplified approach for the stability analysis via the rank-preserving flow theory.

- We have established the exponential stability of rigid formation control systems for a large family of formation controllers in Chapter 4 . The results hold for both minimally rigid formations and non-minimally rigid formations.

\footnotetext{
${ }^{1}$ For experiment verifications relating to some theoretical results of this thesis, we recommend relevant online videos from two YouTube channels maintained and updated by our collaborating groups: (i) https://www.youtube.com/channel/UCY7wTEIqc0-0IQEDjhkIgHg by DCASL (Distributed Control and Autonomous Systems Laboratory) at GIST led by Prof. Hyo-Sung Ahn; and (ii) https://www.youtube.com/user/noeth3r by Dr. Héctor Garcia de Marina.
} 
- An invariance principle on the equilibrium set and its stability for double-integrator formation systems is established in Chapter 8, which provides a link between single-integrator and double-integrator formation systems. To this end, certain results on equilibrium analysis from single-integrator formation systems, such as these discussed in Chapter 3 and in other literature, can be extended to the stability analysis of formation systems modelled by double integrators.

\subsubsection{Formation control systems: some practical considerations}

- From the exponential stability obtained in Chapter 4, in Chapter 5 we have discussed the robustness issue for (3-D) rigid formation control systems in the presence of mismatched distances. We show that generically a helical motion will be induced by small and constant mismatches between neighboring agents in their distance measurements or perceived target distances. Motion parameter formulas are also derived to describe these rigid formation movements.

- In practice, the stabilization of only rigid formation shapes may not be sufficient for real-life applications and the achieving of a certain orientation for a rigid formation could also be desirable. To address this problem, in Chapter 6 we propose a feasible formation controller to achieve a desired rigid shape and a prescribed formation orientation in both ambient 2-D and 3-D spaces. In this control framework, we also prove the minimal number of agents requiring the global knowledge of a common coordinate orientation to achieve this task.

- Motivated by practical sensing and measurement constraints of digital sensors, we discuss the quantization effect on rigid formation control (in Chapter 7). Two types of quantizers are considered, namely the uniform quantizer and the logarithmic quantizer. The convergence property of the formation system and the tradeoff of using quantized measurements are investigated in detail in Chapter 7.

\subsubsection{Distributed coordination control: general system models}

- In Chapter 8, we have discussed in detail two types of double-integrator systems: formation stabilization systems and flocking control systems with a target rigid shape. The measurement requirement, properties of equilibrium set and Jacobian matrix analysis are shown in Chapter 8. By constructing a family of parameterized Hamiltonian-like system, invariance principles concerning the equilibrium set and local stability are also established which help to analyze the stability of different equilibrium sets for formation control systems modelled by double integrators.

- A general framework for distributed coordination control with both formation constraints and kinematics constraints is presented in Chapter 9. The coordination control described by formation constraints includes the rigid formation control as a special case, and also provides a very general framework to model 
other coordination tasks. We consider a general problem setting that each agent is modelled by an affine control system with possible drift terms, and individual agents may have totally different dynamics (thus enabling the heterogeneity in modelling multi-agent systems). Based on this setting, in Chapter 9 we have discussed in detail a fundamental problem in distributed coordination control: the formation feasibility problem. An algebraic condition is provided to determine the existence of feasible formations in such heterogeneous networked systems, and a systematic procedure is proposed to generate feasible trajectories (if they exist) for all the agents.

\subsection{Future work}

In this section we outline some research problems for future research. They are listed according to different topics as shown below.

\subsubsection{Equilibrium analysis}

In Chapter 3 we show a further step to understand the equilibrium property of the gradient-based rigid formation system. We also list some open problems in the future research as follows.

- For formation systems with a general target shape, it has not been determined analytically whether there exist local stable minima ${ }^{2}$. One may start with the special case of a 2-D K4 (complete graph with 4 agents) formation, and determine the uniqueness of local/global minima.

- Apart from the investigation of equilibrium property, it is also worthwhile to propose modified formation controllers to avoid the convergence to incorrect shapes or to guarantee the convergence to the correct formation shape in an almost global sense ${ }^{3}$. We recently proposed a modified control law with a virtual coordinate to achieve almost global stabilization of K4 formations [Park et al., 2016]. We expect that this approach, which bypasses the determination of local/global minima for a general K4 formation, will also enable almost global convergence for general formations modelled by complete graphs in arbitrary dimensions.

\footnotetext{
${ }^{2}$ In the conclusion section of a recent survey paper [Dokmanic et al., 2015] it states: "(On analytical local minimum of s-stress) everyone agrees that there are many, but, to the best of our knowledge, no analytical minimum of s-stress has yet been found." The s-stress function takes exactly the same form as the potential function (2.9) in formation control. Numerical examples of a local minimum of such potentials indeed exist; see our recent paper [Park et al., 2016].

${ }^{3}$ Along this research direction, a recent paper [Tian and Wang, 2013] proposed a modified formation controller to almost globally stabilize a rigid target formation. However, we recently found some incorrect statements in [Tian and Wang, 2013] (see the commentary paper [Trinh et al., 2016]). Thus, the global stabilization of a target rigid formation is still an open problem.
} 
- It is desirable to study other types of invariants apart from the rank and centroid (as discussed in Chapter 3) such that more properties of the formation system can be explored.

- An outstanding issue is to establish the Morse function property when the desired formation is in a $d \geq 2$ dimensional space. It has been established for one-dimensional formations [Anderson and Helmke, 2014] and for complete formations in higher dimensions [Draisma et al., 2015]. The analysis in [Helmke and Anderson, 2013] provided some bounds on the number critical points of two gradient-type formation systems based on the assumption that the potential is an equivariant Morse function. However, it is still unknown whether the potential is generically an equivariant Morse function.

- The general issue is to find algebraic invariants of the gradient flow in terms of computer algebra methods, which may facilitate the analysis with the aid of available computation algorithms.

\subsubsection{Robustness issues}

The robustness issues, and in particular the rigid motions for 3-D formation control systems induced by distance mismatches, are discussed in detail in Chapter 5 . We list some interesting problems along this research direction.

- Quantization is a source of inaccuracy in measurements and/or communications in distributed coordination control. In Chapter 7, we assume the quantization is applied to the distance measurements, and it will be more practical to assume both quantized distance and quantized bearing measurements. It is also interesting to investigate the case that different agents employ nonidentical quantizers in their controllers. This suggests a typical scenario of distance mismatches, but it is not clear whether it gives rise to steady-state rigid motions, as we have observed in [Mou et al., 2016] when agents have unmatched distance measurements.

- It will be interesting to investigate whether the rigid motions, as observed in Chapter 5 on single-integrator formation systems, still occur in doubleintegrator formation systems with inconsistent distances or distance measurement errors. The main tools in Chapter 5 and [Mou et al., 2016] include linearization analysis and exponential stability of the single-integrator system. Thus, we expect that the linearization technique and Jacobian matrix analysis in Section 8.3, as well as the parameterized system analysis and the exponential convergence established in Section 8.4, will be fundamental results to establish the robustness property for double-integrator rigid formation systems. Some results on rigid motions in double-integrator formation systems have been reported in [Garcia de Marina et al., 2016b], which focused on the formation stabilization model. It is still unclear about the additional motions and formation 
properties for double-integrator flocking models with mismatched distances or other perturbations.

- Finally, we remark that such robustness issues on distributed formation control discussed in Chapter 5 also have implications in the general field of cooperative control involving gradient-flow approach, distributed coordination and local information sharing [Sakurama et al., 2015]. This will help to reveal the fundamental performance limitations and robustness behaviors in general coordination control systems.

\subsubsection{Formation feasibility and motion generation}

In Chapter 9 we consider the fundamental problem of determining formation feasibility and how to generate feasible motions for networked heterogeneous systems with drift terms. We also list some open problems for future research along this direction:

- In Chapter 9, the constraints in modelling a global control task and agents' dynamics are described by some equalities. It is worthwhile to extend the formation feasibility theory to the case with inequality constraints. This is partly motivated by the coordination control problem of multiple agents with almost constant speeds (as in [Anderson et al., 2008a]).

- We also note that the motion generation algorithm reported in Chapter 9 assumes that all agents are initially at a feasible trajectory. It will be interesting to develop control approaches to stabilize all agents' trajectories to one of the feasible trajectories if they exist.

\subsubsection{Longer-term research problems: coordination control in nonlinear spaces}

In this thesis the coordination control and formation control have been mostly focused on the real Euclidean space, and only in Chapter 9 have we considered a general problem where agents' states may not be confined to live in the Euclidean space. Such problems can be further generalized, in the sense that agents' states may live in some nonlinear spaces, e.g. on a sphere, a smooth surface, or (more generally) a Riemannian manifold. This has been motivated by many real-life applications in distributed coordination and cooperative control, for example attitude control, orientation synchronization, etc.

Consensus of multi-agent systems has been considered as a fundamental tool in multi-agent distributed control which serves as a basis for many distributed algorithms. There has been an increasing interest in recent years to consider consensus and distributed control in nonlinear spaces (see e.g. [Sepulchre, 2011], [Tron et al., 2013; Li and Spong, 2014; Satici and Spong, 2016]). Recently we have revisited the fundamental consensus problem when agents' states are confined to be living on a sphere [Lageman and Sun, 2016] and have developed a perturbation theory for this 
coordination problem. Based on this and other findings achieved in this thesis, we expect more results on coordination control in nonlinear spaces will be achieved in the future research. 


\section{Bibliography}

Abdessameud, A. And TayebI, A., 2013. Motion Coordination for VTOL Unmanned Aerial Vehicles: Attitude Synchronisation and Formation Control. Springer Science \& Business Media. (cited on page 5)

Abraham, R.; Marsden, J. E.; And Ratiu, T., 1988. Manifolds, Tensor Analysis, and Applications. Second edition. Springer-Verlag. (cited on page 30)

Absil, P.-A. AND KuRdyKa, K., 2006. On the stable equilibrium points of gradient systems. Systems $\mathcal{E}$ control letters, 55, 7 (2006), 573-577. (cited on pages 15 and 82)

Anderson, B. D. O., 2011. Morse theory and formation control. In Proc. of the 19th Mediterranean Conference on Control \& Automation (MED), 656-661. IEEE. (cited on pages 4,16 , and 20)

Anderson, B. D. O.; FidAN, B.; YU, C.; AND WALle, D., 2008a. UAV formation control: theory and application. In Recent advances in learning and control, 15-33. Springer. (cited on pages 6, 144, and 157)

Anderson, B. D. O. And Helmke, U., 2014. Counting critical formations on a line. SIAM Journal on Control and Optimization, 52, 1 (2014), 219-242. (cited on pages 4, $16,19,20,27,28,36,39,73,82,113,120,122$, and 156)

Anderson, B. D. O.; Yu, C.; Dasgupta, S.; And Morse, A. S., 2007. Control of a threecoleader formation in the plane. Systems $\mathcal{E}$ Control Letters, 56, 9 (2007), 573-578. (cited on pages 36,38 , and 95)

Anderson, B. D. O.; Yu, C.; Dasgupta, S.; And Summers, T. H., 2010. Controlling four agent formations. In Proc. of the 2nd IFAC Workshop on Distributed Estimation and Control in Networked Systems, 139-144. (cited on page 20)

Anderson, B. D. O.; Yu, C.; Fidan, B.; And Hendrickx, J. M., 2008b. Rigid graph control architectures for autonomous formations. Control Systems Magazine, IEEE, 28, 6 (2008), 48-63. (cited on pages 14, 25, 36, and 121)

Aranda, M.; López-Nicolás, G.; Sagüés, C.; and Zavlanos, M. M., 2015. Coordinate-free formation stabilization based on relative position measurements. Automatica, 57 (2015), 11-20. (cited on page 3)

Asimow, L. AND Roth, B., 1978. The rigidity of graphs. Transactions of the American Mathematical Society, 245 (1978), 279-289. (cited on page 10) 
Asimow, L. AND Roth, B., 1979. The rigidity of graphs, II. Journal of Mathematical Analysis and Applications, 68, 1 (1979), 171-190. (cited on pages 4 and 11)

Bacciotti, A. and Ceragioli, F., 1999. Stability and stabilization of discontinuous systems and nonsmooth lyapunov functions. ESAIM: Control, Optimisation and Calculus of Variations, 4 (1999), 361-376. (cited on pages 109 and 110)

Baillieul, J. AND Antsaklis, P. J., 2007. Control and communication challenges in networked real-time systems. Proceedings of the IEEE, 95, 1 (2007), 9-28. (cited on page 56)

Baillieul, J. And Samad, T., 2015. Encyclopedia of Systems and Control. Springer Publishing Company. (cited on page 1)

BAIllieul, J. AND SURI, A., 2003. Information patterns and hedging brockett's theorem in controlling vehicle formations. In Proc. of the 42nd IEEE Conference on Decision and Control, 556-563. (cited on page 56)

BAPAT, R. B., 2010. Graphs and Matrices. Springer. (cited on page 10)

Belabbas, A.; Mou, S.; Morse, A. S.; and Anderson, B. D. O., 2012. Robustness issues with undirected formations. In Proc. of the 51st IEEE Conference on Decision and Control, 1445-1450. (cited on pages 4, 36, 39, 56, and 61)

Bishop, A. N.; Deghat, M.; Anderson, B. D. O.; And Hong, Y., 2015. Distributed formation control with relaxed motion requirements. International Journal of Robust and Nonlinear Control, 25, 17 (2015), 3210-3230. (cited on page 46)

Borg, I. And Groenen, P. J., 2005. Modern Multidimensional Scaling: Theory and Applications. Springer Science \& Business Media. (cited on pages 20 and 32)

Brockett, R. W., 2014. The early days of geometric nonlinear control. Automatica, 50, 9 (2014), 2203-2224. (cited on page 140)

BRockett, R. W. AND Liberzon, D., 2000. Quantized feedback stabilization of linear systems. IEEE Transactions on Automatic Control, 45, 7 (2000), 1279-1289. (cited on page 91)

CAI, K. AND IsHII, H., 2011. Quantized consensus and averaging on gossip digraphs. IEEE Transactions on Automatic Control, 56, 9 (2011), 2087-2100. (cited on pages 91 and 93)

CAI, X. ANd De QueIroz, M., 2014. Multi-agent formation maneuvering and target interception with double-integrator model. In Proc. of the American Control Conference, 287-292. IEEE. (cited on page 114)

CAI, X. AND DE QueIROZ, M., 2014. Rigidity-based stabilization of multi-agent formations. Journal of Dynamic Systems, Measurement, and Control, 136, 1 (2014), 014502. (cited on page 39) 
CAI, X. AND De QueIroz, M., 2015. Adaptive rigidity-based formation control for multirobotic vehicles with dynamics. IEEE Transactions on Control Systems Technology, 23, 1 (2015), 389-396. (cited on pages 4, 39, and 73)

CaO, M.; Morse, A. S.; Yu, C.; Anderson, B. D. O.; And Dasgupta, S., 2011. Maintaining a directed, triangular formation of mobile autonomous agents. Communications in Information and Systems, 11, 1 (2011), 1-16. (cited on pages 4, 16, 20, 24, $42,45,80$, and 128)

CaO, Y.; Yu, W.; Ren, W.; and Chen, G., 2013. An overview of recent progress in the study of distributed multi-agent coordination. IEEE Transactions on Industrial Informatics, 9, 1 (2013), 427-438. (cited on pages 1, 5, 113, and 137)

CARli, R.; Bullo, F.; ANd Zampieri, S., 2010. Quantized average consensus via dynamic coding/decoding schemes. International Journal of Robust and Nonlinear Control, 20, 2 (2010), 156-175. (cited on pages 93 and 94)

Carlson, D. ANd Schneider, H., 1963. Inertia theorems for matrices: The semidefinite case. Journal of Mathematical Analysis and Applications, 6, 3 (1963), 430-446. (cited on pages 9 and 126)

CARr, J., 1981. Applications of Centre Manifold Theory. Springer. (cited on pages 30, 122 , and 130)

Ceragioli, F.; De Persis, C.; And Frasca, P., 2011. Discontinuities and hysteresis in quantized average consensus. Automatica, 47, 9 (2011), 1916-1928. (cited on pages 91, 93, and 95)

Chen, X., 2014. Multi-agent Systems with Reciprocal Interaction Laws. Ph.D. thesis, Harvard University. Supervisor: Prof. Roger. W. Brockett. (cited on page 20)

Chen, X.; Belabbas, M.-A.; AND BAŞAR, T., 2015. Formation control with triangulated laman graphs. Available at arXiv preprint arXiv:1412.6958, (2015). (cited on page 119)

Chen, X. And Brockett, R. W., 2014. Centralized and decentralized formation control with controllable interaction laws. In Proc. of the 53rd IEEE Conference on Decision and Control, 601-606. (cited on page 23)

Chinng, H.-D. and Chu, C.-C., 1995. Theoretical foundation of the BCU method for direct stability analysis of network-reduction power system models with small transfer conductances. IEEE Transactions on Circuits and Systems I: Fundamental Theory and Applications, 42, 5 (1995), 252-265. (cited on pages 114, 124, 125, and 130)

Chiang, H.-D. and Wu, F. F., 1988. Stability of nonlinear systems described by a second-order vector differential equation. IEEE Transactions on Circuits and Systems, 35, 6 (1988), 703-711. (cited on pages 114, 124, and 125) 
CHILl, R., 2003. On the Łojasiewicz-Simon gradient inequality. Journal of Functional Analysis, 201, 2 (2003), 572-601. (cited on page 43)

Clarke, F. H., 1998. Nonsmooth Analysis and Control Theory. Springer Science \& Business Media. (cited on pages 108 and 109)

Clarke, F. H., 2013. Functional Analysis, Calculus of Variations and Optimal Control, vol. 264. Springer Science \& Business Media. (cited on page 99)

Connelly, R., 2005. Generic global rigidity. Discrete E Computational Geometry, 33, 4 (2005), 549-563. (cited on page 22)

Connelly, R. And Guest, S. D., 2015. Frameworks, Tensegrities and Symmetry: Understanding Stable Structures. http://www.math.cornell.edu/ web7510/framework.pdf. (cited on page 11)

CoRTÉs, J., 2006. Finite-time convergent gradient flows with applications to network consensus. Automatica, 42, 11 (2006), 1993-2000. (cited on page 110)

CoRTÉs, J., 2008. Discontinuous dynamical systems. Control Systems Magazine, IEEE, 28, 3 (2008), 36-73. (cited on pages 95, 99, 102, 109, and 110)

Cortés, J., 2009. Global and robust formation-shape stabilization of relative sensing networks. Automatica, 45, 12 (2009), 2754-2762. (cited on pages 3, 16, 59, and 73)

Dasgupta, S.; Anderson, B. D. O.; Yu, C.; and Summers, T. H., 2011. Controlling rectangular formations. In Prof. of the 2011 Australian Control Conference (AUCC), 44-49. IEEE. (cited on page 4)

De Persis, C.; Liv, H.; and CaO, M., 2010. Control of one-dimensional guided formations using coarsely quantized information. In Proc. of the 49th IEEE Conference on Decision and Control (CDC), 2257-2262. IEEE. (cited on pages 5 and 101)

Deghat, M.; Anderson, B. D. O.; AND Lin, Z., 2016. Combined flocking and distancebased shape control of multi-agent formations. IEEE Transactions on Automatic Control, 61, 7 (2016), 1824-1837. (cited on pages 5, 114, 116, 120, 121, 122, 128, 131, and 132)

Dimarogonas, D. V. AND Johansson, K. H., 2008. On the stability of distance-based formation control. In Proc. of the 47th Conference on Decision and Control, 1200-1205. IEEE. (cited on pages 39, 113, 114, 121, and 131)

Dimarogonas, D. V. And Johansson, K. H., 2009. Further results on the stability of distance-based multi-robot formations. In Proc. of American Control Conference, 2972-2977. IEEE. (cited on page 4)

Dimarogonas, D. V. AND Johansson, K. H., 2010. Stability analysis for multi-agent systems using the incidence matrix: quantized communication and formation control. Automatica, 46, 4 (2010), 695-700. (cited on pages 16, 19, 36, 39, and 59) 
Dimarogonas, D. V. And Kyriakopoulos, K. J., 2008. A connection between formation infeasibility and velocity alignment in kinematic multi-agent systems. Automatica, 44, 10 (2008), 2648-2654. (cited on page 141)

Dokmanic, I.; PARhizkar, R.; Ranieri, J.; AND Vetterli, M., 2015. Euclidean distance matrices: essential theory, algorithms, and applications. IEEE Signal Processing Magazine, 32, 6 (2015), 12-30. (cited on page 155)

DonG, X., 2015. Formation and Containment Control for High-order Linear Swarm Systems. Springer Theses, Springer-Verlag Berlin Heidelberg. (cited on page 5)

Dörfler, F. AND Bullo, F., 2011. On the critical coupling for kuramoto oscillators. SIAM Journal on Applied Dynamical Systems, 10, 3 (2011), 1070-1099. (cited on pages $114,125,126,129$, and 130)

Dörfler, F. AND Francis, B., 2009. Formation control of autonomous robots based on cooperative behavior. In Proc. of the 2009 European Control Conference, 2432-2437. IEEE. (cited on page 38)

DörfLER, F. AND FrANCIS, B., 2010. Geometric analysis of the formation problem for autonomous robots. IEEE Transactions on Automatic Control, 55, 10 (2010), 23792384. (cited on pages $4,16,20,36,39,59,73,113,114,127,128,129$, and 131)

Draisma, J.; Horobeţ, E.; Ottaviani, G.; Sturmfels, B.; and Thomas, R. R., 2015. The euclidean distance degree of an algebraic variety. Foundations of Computational Mathematics, (2015), 1-51. (cited on page 156)

Egerstedt, M. AND Hu, X., 2001. Formation constrained multi-agent control. IEEE Transactions on Robotics and Automation, 17, 6 (2001), 947-951. (cited on page 138)

ElkiN, V. I., 2012. Reduction of Nonlinear Control Systems: A Differential Geometric Approach, vol. 472. Springer Science \& Business Media. (cited on page 144)

Eren, T.; Anderson, B. D. O.; Morse, A. S.; Whiteley, W.; Belhumeur, P. N.; et Al., 2003. Operations on rigid formations of autonomous agents. Communications in Information \& Systems, 3, 4 (2003), 223-258. (cited on page 10)

Fahimi, F., 2008. Autonomous Robots: Modeling, Path Planning, and Control, vol. 107. Springer Science \& Business Media. (cited on page 2)

Filippov, A. F., 1988. Differential Equations with Discontinuous Righthand Sides. Springer Science \& Business Media. (cited on page 95)

Francis, B. A. And Maggiore, M., 2016. Flocking and Rendezvous in Distributed Robotics. Springer. (cited on page 5)

Frasca, P., 2012. Continuous-time quantized consensus: convergence of Krasovskii solutions. Systems $\mathcal{E}$ Control Letters, 61, 2 (2012), 273-278. (cited on pages 91 and 93) 
Garcia de Marina, H.; CaO, M.; and Jayawardhana, B., 2015. Controlling rigid formations of mobile agents under inconsistent measurements. IEEE Transactions on Robotics, 31, 1 (2015), 31-39. (cited on pages 36 and 71)

Garcia de Marina, H.; Jayawardhana, B.; and CaO, M., 2016a. Distributed rotational and translational maneuvering of rigid formations and their applications. IEEE Transactions on Robotics, 32, 3 (2016), 684-697. (cited on pages 71 and 75)

Garcia de Marina, H.; Jayawardhana, B.; and CaO, M., 2016b. Taming interdistance mismatches in formation-motion control for rigid formations of secondorder agents. IEEE Transactions on Automatic Control, submitted. Availabe at Arxiv: http://arxiv.org/abs/1604.02943, (2016). (cited on pages 95, 100, 131, and 156)

Gregory, R. D., 2006. Classical Mechanics. Cambridge University Press. (cited on pages 62,63 , and 68 )

Guler, S.; KoKSAL, N.; AND FIDAN, B., 2013. Adaptive control of a three-agent surveillance swarm with constant speed constraint. In Proc. of the 9th Asian Control Conference (ASCC), 1-6. IEEE. (cited on page 144)

Guo, M. and Dimarogonas, D. V., 2013. Consensus with quantized relative state measurements. Automatica, 49, 8 (2013), 2531-2537. (cited on pages 91 and 93)

Hahn, W. ANd BaARTz, A. P., 1967. Stability of Motion, vol. 138. Springer. (cited on page 120)

Helmke, U. And Anderson, B. D. O., 2013. Equivariant morse theory and formation control. In Proc. of the 51st Annual Allerton Conference on Communication, Control, and Computing, 1576-1583. IEEE. (cited on pages 4, 20, 26, 27, 113, 119, and 156)

Helmke, U. ANd Moore, J. B., 1994. Optimization and Dynamical Systems. Springer. (cited on pages 32,33 , and 129)

Helmke, U.; Mou, S.; Sun, Z.; And Anderson, B. D. O., 2014. Geometrical methods for mismatched formation control. In Proc. of the 53rd IEEE Conference on Decision and Control, 1341-1346. (cited on pages 23 and 56)

HeNDRICKSON, B., 1992. Conditions for unique graph realizations. SIAM Journal on Computing, 21, 1 (1992), 65-84. (cited on pages 11, 12, 44, 46, 105, 115, and 121)

HendRICKx, J., 2008. Graphs and Networks for the Analysis of Autonomous Agent Systems. Ph.D. thesis, Université catholique de Louvain, Supervisor: Prof. Vincent Blondel. (cited on pages 12 and 14)

Horn, R. A. And Johnson, C. R., 2012. Matrix Analysis. Cambridge University Press. (cited on page 48)

IsIDORI, A., 1995. Nonlinear Control Systems. Springer Science \& Business Media. (cited on pages 6, 138, 139, and 140) 
Jafarian, M. ANd De Persis, C., 2015. Formation control using binary information. Automatica, 53, 0 (2015), $125-135$. (cited on pages 5 and 92)

Jiang, B.; Deghat, M.; ANd Anderson, B. D. O., 2017. Simultaneous velocity and position estimation via distance-only measurements with application to multi-agent system control. IEEE Transactions on Automatic Control, in press, 62, 2 (2017), 869875. (cited on page 131)

Justh, E. W. AND KRISHNAPRASAD, P. S., 2005. Natural frames and interacting particles in three dimensions. In Proc. of the 44th IEEE Conference on Decision and Control and 2005 European Control Conference., 2841-2846. IEEE. (cited on page 57)

Kashyap, A.; Başar, T.; ANd SRIKant, R., 2007. Quantized consensus. Automatica, 43, 7 (2007), 1192-1203. (cited on page 91)

Khalil, H. K., 2002. Nonlinear systems, Third Edition. Prentice Hall, New Jersey. (cited on pages $36,41,48,49,82$, and 84 )

Knorn, S.; Chen, Z.; And Middleton, R., 2016. Overview: Collective control of multi-agent systems. IEEE Transactions on Control of Network Systems, 3, 4 (2016), 334-347. (cited on pages 1, 5, and 137)

KoditscheK, D. E. AND Rimon, E., 1990. Robot navigation functions on manifolds with boundary. Advances in Applied Mathematics, 11, 4 (1990), 412-442. (cited on page 20)

Krick, L.; BroucKe, M. E.; AND Francis, B. A., 2009. Stabilisation of infinitesimally rigid formations of multi-robot networks. International Journal of Control, 82, 3 (2009), 423-439. (cited on pages 4, 9, 16, 19, 22, 25, 36, 39, 59, 73, 80, 92, 98, 113, $114,118,127,128,130$, and 145)

Lageman, C., 2007. Convergence of Gradient-like Dynamical Systems and Optimization Algorithms. Ph.D. thesis, University of Würzburg, Supervisor: Prof. Uwe Helmke. (cited on page 15)

Lageman, C. And Sun, Z., 2016. Consensus on spheres: convergence analysis and perturbation theory. In Proc. of the 55th IEEE Conference on Decision and Control, 19-24. (cited on page 157)

LEE, J. M., 2009. Manifolds and Differential Geometry, vol. 107. American Mathematical Society. (cited on page 70)

LI, W. AND SPOnG, M. W., 2014. Unified cooperative control of multiple agents on a sphere for different spherical patterns. IEEE Transactions on Automatic Control, 59, 5 (2014), 1283-1289. (cited on page 157)

Liberzon, D., 2003. Hybrid feedback stabilization of systems with quantized signals. Automatica, 39, 9 (2003), 1543-1554. (cited on page 91) 
LIN, Z.; WANG, L.; CHEN, Z.; FU, M.; AND HAN, Z., 2016a. Necessary and sufficient graphical conditions for affine formation control. IEEE Transactions on Automatic Control, 61, 10 (2016), 2877-2891. (cited on page 74)

LiN, Z.; WANG, L.; HAN, Z.; AND FU, M., 2016b. A graph laplacian approach to coordinate-free formation stabilization for directed networks. IEEE Transactions on Automatic Control, 61, 5 (2016), 1269-1280. (cited on page 3)

LiU, H.; CaO, M.; and De Persis, C., 2012. Quantization effects on synchronized motion of teams of mobile agents with second-order dynamics. Systems $\mathcal{E}$ Control Letters, 61, 12 (2012), 1157-1167. (cited on pages 91, 93, 99, 104, and 105)

LiU, H.; de Marina, H. G.; ANd CaO, M., 2014. Controlling triangular formations of autonomous agents in finite time using coarse measurements. In Proc. of IEEE International Conference on Robotics and Automation (ICRA), 3601-3606. IEEE. (cited on pages 5,92 , and 101 )

Liv, K.; Fridman, E.; AND Johansson, K. H., 2015. Dynamic quantization of uncertain linear networked control systems. Automatica, 59 (2015), 248-255. (cited on page 91)

Maithripala, D.; Maithripala, D.; And Jayasuriya, S., 2008. Unifying geometric approach to real-time formation control. In Proc. of the 2008 American Control Conference, 789-794. IEEE. (cited on page 138)

Markdahl, J.; Karayiannidis, Y.; Hu, X.; and Kragic, D., 2012. Distributed cooperative object attitude manipulation. In Proc. of IEEE International Conference on Robotics and Automation (ICRA), 2960-2965. IEEE. (cited on page 74)

MeIss, J. D., 2007. Differential Dynamical Systems, vol. 14. SIAM. (cited on pages 128, 129 , and 130)

Meng, Z.; Anderson, B. D. O.; ANd Hirche, S., 2016. Formation control with mismatched compasses. Automatica, 69 (2016), 232-241. (cited on pages 3 and 92)

Mesbahi, M. ANd Egerstedt, M., 2010. Graph Theoretic Methods in Multiagent Networks. Princeton University Press. (cited on pages 10, 77, and 138)

Montijano, E.; Cristofalo, E.; Zhou, D.; Schwager, M.; and SagüÉs, C., 2016. Vision-based distributed formation control without an external positioning system. IEEE Transactions on Robotics, 32, 2 (2016), 339-351. (cited on page 3)

MontiJano, E.; Zhou, D.; SCHwager, M.; AND SAgüÉs, C., 2014. Distributed formation control without a global reference frame. In Proc. of the 2014 American Control Conference, 3862-3867. (cited on page 74)

Mou, S.; Belabbas, M. A.; Morse, A. S.; Sun, Z.; and Anderson, B. D. O., 2016. Undirected rigid formations are problematic. IEEE Transactions on Automatic Control, in press, 61, 10 (2016), 2821-2836. (cited on pages 4, 7, 14, 23, 36, 41, 44, 46, 48, $52,56,58,59,61,62,66,70,84,131$, and 156) 
Mou, S.; CAO, M.; ANd Morse, A. S., 2015. Target-point formation control. Automatica, 61 (2015), 113-118. (cited on page 35)

Mou, S.; Morse, A. S.; ANd Anderson, B. D. O., 2014. Toward robust control of minimally rigid undirected formations. In Proc. of the 53rd IEEE Conference on Decision and Control, 643-647. (cited on page 71)

Murray, R. M.; LI, Z.; And Sastry, S. S., 1994. A Mathematical Introduction to Robotic Manipulation. CRC press. (cited on page 139)

OH, K.-K. AND AHN, H.-S., 2011. Formation control of mobile agents based on interagent distance dynamics. Automatica, 47, 10 (2011), 2306-2312. (cited on page 73)

OH, K.-K. AND AHN, H.-S., 2014a. Distance-based undirected formations of singleintegrator and double-integrator modeled agents in n-dimensional space. International Journal of Robust and Nonlinear Control, 24, 12 (2014), 1809-1820. (cited on pages $4,5,16,43,59,80,114,125,126$, and 131)

OH, K. K. AND AhN, H. S., 2014b. Formation control and network localization via orientation alignment. IEEE Transactions on Automatic Control, 59, 2 (2014), 540-545. (cited on pages 3 and 74)

OH, K.-K.; PARK, M.-C.; ANd AhN, H.-S., 2015. A survey of multi-agent formation control. Automatica, 53 (2015), 424-440. (cited on pages 2, 3, 5, 35, 77, 92, 113, 118, 128,138 , and 141)

Olfati-Saber, R., 2006. Flocking for multi-agent dynamic systems: Algorithms and theory. IEEE Transactions on Automatic Control, 51, 3 (2006), 401-420. (cited on pages 5 and 113)

Olfati-Saber, R. And Murray, R. M., 2002. Graph rigidity and distributed formation stabilization of multi-vehicle systems. In Proc. of the 41st Conference on Decision and Control, 2965-2971. IEEE. (cited on pages 4 and 36)

PAden, B. AND SAstry, S., 1987. A calculus for computing Filippov's differential inclusion with application to the variable structure control of robot manipulators. IEEE Transactions on Circuits and Systems, 34, 1 (1987), 73-82. (cited on page 108)

Pais, D.; CaO, M.; And Leonard, N. E., 2009. Formation shape and orientation control using projected collinear tensegrity structures. In Proc. of the 2009 American Control Conference, 610-615. IEEE. (cited on page 74)

PARK, M.-C. AND AHN, H.-S., 2014. Exponential stabilization of infinitesimally rigid formations. In Proc. of the 2014 International Conference on Control, Automation and Information Sciences, 36-40. IEEE. (cited on page 46) 
Park, M.-C.; Jeong, K.; AND AhN, H.-S., 2013. Control of undirected four-agent formations in 3-dimensional space. In Proc. of the 52nd IEEE Conference on Decision and Control, 1461-1465. IEEE. (cited on page 128)

Park, M.-C.; Sun, Z.; Anderson, B. D. O.; ANd Ahn, H.-S., 2014. Stability analysis on four agent tetrahedral formations. In Proc. of the 53rd IEEE Conference on Decision and Control, 631-636. (cited on pages 4, 20, 24, 114, 127, and 129)

Park, M.-C.; Sun, Z.; Trinh, M. H.; Anderson, B. D. O.; and Ahn, H.-S., 2016. Distance-based control of $\mathrm{K} 4$ formation with almost global convergence. In Proc. of the 55th IEEE Conference on Decision and Control, 904-909. IEEE. (cited on pages 27 and 155)

Persis, C. D. AND Frasca, P., 2013. Robust self-triggered coordination with ternary controllers. IEEE Transactions on Automatic Control, 58, 12 (2013), 3024-3038. (cited on page 103)

QIN, J. AND YU, C., 2013. Coordination of multiagents interacting under independent position and velocity topologies. IEEE Transactions on Neural Networks and Learning Systems, 24, 10 (2013), 1588-1597. (cited on page 116)

Ramazani, S.; Selmic, R. R.; AND De QueIroz, M., 2015. Stabilization of non-planar multi-agent layered formations with double integrator model. In Proc. of the IEEE Conference on Control Applications, 1386-1391. IEEE. (cited on page 114)

REN, W., 2008. On consensus algorithms for double-integrator dynamics. IEEE Transactions on Automatic Control, 53, 6 (2008), 1503-1509. (cited on page 113)

Ren, W. and Beard, R. W., 2008. Distributed Consensus in Multi-vehicle Cooperative Control. Springer. (cited on page 2)

Ren, W. And CaO, Y., 2010. Distributed Coordination of Multi-agent Networks: Emergent Problems, Models, and Issues. Springer Science \& Business Media. (cited on page 1)

RozenHeCK, O.; ZhaO, S.; AND Zelazo, D., 2015. A proportional-integral controller for distance-based formation tracking. In Proc. of the 2015 European Control Conference, 1687-1692. (cited on page 113)

SaKurama, K.; Azuma, S.; and Sugie, T., 2015. Distributed controllers for multiagent coordination via gradient-flow approach. IEEE Transactions on Automatic Control, 60, 6 (2015), 1471-1485. (cited on pages 37 and 157)

SATici, A. C. AND SPong, M. W., 2016. Global swarming while preserving connectivity via lagrange-poincarè equations. Automatica, 71 (2016), 369-380. (cited on page 157)

Scardovi, L.; LeONARD, N. E.; AND Sepulchre, R., 2008. Stabilization of threedimensional collective motion. Communications in Information $\mathcal{E}$ Systems, 8, 4 (2008), 473-500. (cited on page 57) 
Sepulchre, R., 2011. Consensus on nonlinear spaces. Annual reviews in control, 35, 1 (2011), 56-64. (cited on page 157)

Sepulchre, R.; Paley, D.; ANd Leonard, N. E., 2008. Stabilization of planar collective motion with limited communication. IEEE Transactions on Automatic Control, 53, 3 (2008), 706-719. (cited on pages 57, 138, and 144)

Seyboth, G. S.; Wu, J.; Qin, J.; Yu, C.; ANd Allgower, F., 2014. Collective circular motion of unicycle type vehicles with nonidentical constant velocities. IEEE Transactions on Control of Network Systems, 1, 2 (2014), 167-176. (cited on pages 138 and 144)

Shevitz, D.; PAden, B.; ET AL., 1994. Lyapunov stability theory of nonsmooth systems. IEEE Transactions on Automatic Control, 39, 9 (1994), 1910-1914. (cited on page 110)

Smith, S. L.; Broucke, M. E.; ANd Francis, B. A., 2006. Stabilizing a multi-agent system to an equilateral polygon formation. In Proc. of the 17th International Symposium on Mathematical Theory of Networks and Systems, 2415-2424. (cited on pages 36 and 38)

Summers, T. H.; Yu, C.; Dasgupta, S.; And Anderson, B. D. O., 2011. Control of minimally persistent leader-remote-follower and coleader formations in the plane. IEEE Transactions on Automatic Control, 56, 12 (2011), 2778-2792. (cited on page 4)

Summers, T. H.; Yu, C.; Dasgupta, S.; and Anderson, B. D. O., 2013. Certifying non-existence of undesired locally stable equilibria in formation shape control problems. In Proc. of the 2013 IEEE International Symposium on Intelligent Control (ISIC), 200-205. IEEE. (cited on pages 20 and 26)

Sun, Z.; Helmke, U.; ANd Anderson, B. D. O., 2015a. Rigid formation shape control in general dimensions: an invariance principle and open problems. In Proc. of the 54th IEEE Conference on Decision and Control, 6095 - 6100. (cited on pages 114, 120, $122,127,128,129$, and 133)

Sun, Z.; Mou, S.; Anderson, B. D. O.; ANd CaO, M., 2016a. Exponential stability for formation control systems with generalized controllers: A unified approach. Systems \& Control Letters, 93 (2016), 50-57. (cited on pages 37, 82, 92, 95, 98, 100, 117 , and 131)

Sun, Z.; Mou, S.; Anderson, B. D. O.; ANd Morse, A. S., 2013. Non-robustness of gradient control for 3-D undirected formations with distance mismatch. In Proc. of the 3rd Australian Control Conference, 369-374. IEEE. (cited on pages 4, 56, 59, 61, 65 , and 70)

Sun, Z.; Mou, S.; Anderson, B. D. O.; And Morse, A. S., 2014a. Formation movements in minimally rigid formation control with mismatched mutual distances. In Proc. of the 53rd IEEE Conference on Decision and Control, 6161-6166. IEEE. (cited on pages 70 and 74 ) 
Sun, Z.; Mou, S.; Anderson, B. D. O.; And Morse, A. S., 2015b. Rigid motions of 3-D undirected formations with distance mismatch. IEEE Transactions on Automatic Control, submitted, (2015). (cited on pages 36, 44, and 52)

Sun, Z.; Mou, S.; Deghat, M.; And Anderson, B. D. O., 2016b. Finite time distributed distance-constrained shape stabilization and flocking control for d-dimensional undirected rigid formations. International Journal of Robust and Nonlinear Control, 26, 13 (2016), 2824-2844. (cited on pages 43 and 121)

Sun, Z.; Mou, S.; Deghat, M.; Anderson, B. D. O.; And Morse, A. S., 2014b. Finite time distance-based rigid formation stabilization and flocking. In Proc. of the 19th IFAC World Congress. 9183-9189. (cited on pages 45, 114, 121, 122, and 131)

Sun, Z.; Mou, S.; Helmke, U.; ANd Anderson, B. D. O., 2014c. Convergence analysis for rigid formation control with unrealizable shapes: The 3 agent case. In Proc. of the 2014 Australian Control Conference, 1-6. (cited on pages 23, 28, and 141)

Sun, Z.; PArK, M.-C.; Anderson, B. D. O.; And Ahn, H.-S., 2017. Distributed stabilization control of rigid formations with prescribed orientation. Automatica. Full version available at http://arxiv.org/abs/1606.03199, 78 (2017), 250 - 257. (cited on pages 76,78 , and 89 )

Sun, Z.; Seyboth, G. S.; AND Anderson, B. D. O., 2015c. Collective control of multiple unicycle agents with non-identical constant speeds: Tracking control and performance limitation. In Proc. of 2015 IEEE Multi-Conference on Systems and Control (MSC'15), 1361-1366. IEEE. (cited on pages 138 and 144)

Tabuada, P.; Pappas, G. J.; AND Lima, P., 2005. Motion feasibility of multi-agent formations. IEEE Transactions on Robotics, 21, 3 (2005), 387-392. (cited on pages 6, $138,140,144,148$, and 149)

Tanner, H. G.; Jadbabaie, A.; and Pappas, G. J., 2007. Flocking in fixed and switching networks. IEEE Transactions on Automatic Control, 52, 5 (2007), 863-868. (cited on pages 5 and 113)

TAY, T.-S. AND Whiteley, W., 1985. Generating isostatic frameworks. Structural Topology 1985, 11 (1985), 21-49. (cited on page 76)

TIAN, Y.-P. AND WANG, Q., 2013. Global stabilization of rigid formations in the plane. Automatica, 49, 5 (2013), 1436-1441. (cited on pages 36, 39, 73, 113, and 155)

Trinh, M. H.; Pham, V. H. P.; Park, M.-C.; Sun, Z.; Anderson, B. D. O.; And Ahn, H.-S., 2016. Comments on 'Global stabilization of rigid formations in the plane'. Automatica, (2016), in press. (cited on page 155)

Tron, R.; AfsarI, B.; AND VidAL, R., 2013. Riemannian consensus for manifolds with bounded curvature. IEEE Transactions on Automatic Control, 58, 4 (2013), 921-934. (cited on page 157) 
Tron, R.; Thomas, J.; Loianno, G.; Danillidis, K.; and Kumar, V., 2016. A distributed optimization framework for localization and formation control: Applications to vision-based measurements. IEEE Control Systems, 36, 4 (Aug 2016), 22-44. (cited on page 3)

Vasile, C.-I.; Schwager, M.; ANd Belta, C., 2015. SE(N) Invariance in Networked Systems. In Proc. of the 2015 European Control Conference (ECC'15), 186-191. IEEE. (cited on pages $3,45,98$, and 118)

WANG, L.; MARKDAHL, J.; AND Hu, X., 2011. Distributed attitude control of multiagent formations. In Proc. of the 18th IFAC World Congress, 2965-2971. (cited on page 74 )

Wiggins, S., 2003. Introduction to Applied Nonlinear Dynamical Systems and Chaos. Springer Science \& Business Media. (cited on pages 15, 24, and 120)

XiaO, F.; Wang, L.; Chen, J.; ANd GaO, Y., 2009. Finite-time formation control for multi-agent systems. Automatica, 45, 11 (2009), 2605-2611. (cited on page 2)

You, K.; XIAO, N.; AND XIE, L., 2015. Analysis and Design of Networked Control Systems. Springer. (cited on page 5)

Yu, C. ANd Anderson, B. D. O., 2009. Development of redundant rigidity theory for formation control. International Journal of Robust and Nonlinear Control, 19, 13 (2009), 1427-1446. (cited on page 75)

Yu, C.; Anderson, B. D. O.; Dasgupta, S.; And Fidan, B., 2009. Control of minimally persistent formations in the plane. SIAM Journal on Control and Optimization, 48, 1 (2009), 206-233. (cited on page 4)

Zelazo, D.; Franchi, A.; Bülthoff, H. H.; and Robuffo Giordano, P., 2015. Decentralized rigidity maintenance control with range measurements for multi-robot systems. The International Journal of Robotics Research, 34, 1 (2015), 105-128. (cited on page 76 )

Zhang, F., 2006. The Schur Complement and its Applications, vol. 4. Springer Science \& Business Media. (cited on page 125)

Zhang, F., 2011. Matrix Theory: Basic Results and Techniques. Springer Science \& Business Media. (cited on pages 84 and 88)

Zhang, F.; EnNasr, O.; Litchman, E.; And Tan, X., 2016. Autonomous sampling of water columns using gliding robotic fish: Algorithms and harmful-algae-sampling experiments. IEEE Systems Journal, 10, 3 (2016), 1271-1281. (cited on page 57)

ZhANG, F.; ZhANG, F.; AND TAN, X., 2014. Tail-enabled spiraling maneuver for gliding robotic fish. Journal of Dynamic Systems, Measurement, and Control, 136, 4 (2014), 041028. (cited on page 57) 
ZhanG, L.; GAO, H.; AND KaYNAK, O., 2013. Network-induced constraints in networked control systems-a survey. IEEE Transactions on Industrial Informatics, 9, 1 (2013), 403-416. (cited on page 1)

Zhang, P.; De QueIroz, M.; AND CAI, X., 2015. Three-dimensional dynamic formation control of multi-agent systems using rigid graphs. Journal of Dynamic Systems, Measurement, and Control, 137, 11 (2015), 111006. (cited on pages 5, 114, and 131)

Zhao, S.; Lin, F.; Peng, K.; Chen, B. M.; and Lee, T. H., 2014. Finite-time stabilisation of cyclic formations using bearing-only measurements. International Journal of Control, 87, 4 (2014), 715-727. (cited on page 101)

ZhaO, S. AND Zelazo, D., 2016. Bearing rigidity and almost global bearing-only formation stabilization. IEEE Transactions on Automatic Control, 61, 5 (2016), 12551268. (cited on page 3) 